THE BOTE MODEL: AN ANALYTIC APPROACH TO PREDICTING GROUND MOTION PHENOMENA RESULTING FROM UNDERGROUND NUCLEAR EXPLOSIONS

\author{
S. R. Kurtz
}

November 9, 1973

Prepared for U.S. Atomic Energy Commission under contract No. W-7405-Eng-48
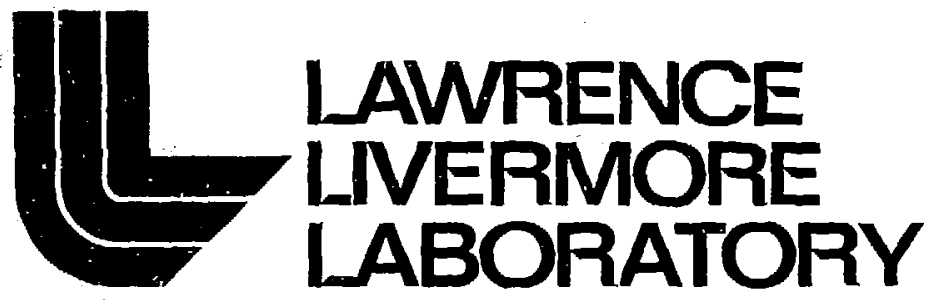

University of Califomia/Livermore 
NOTKC:

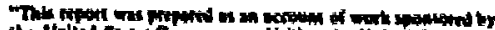

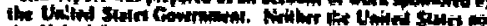

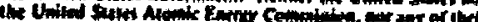

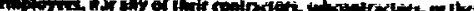

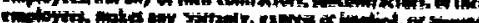

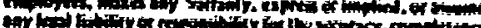

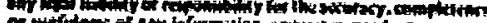

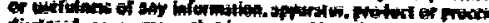

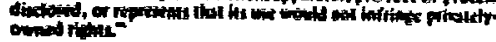

Priated in the United States of America Avallable from

National Technical Information Service

U.S. Department of Commerce 5285 Port Royal Road Springtield, Virginia 22151

Price: Printed Copy $\$$; Microfiche $\$ 0.95$

\begin{tabular}{c} 
* Pages \\
\hline $\begin{array}{c}1-50 \\
51-150 \\
151-325 \\
326-500 \\
501-1000\end{array}$
\end{tabular}

NTIS

Selling Price

$\$ 4.00$

$\$ 5.45$

$\$ 7.60$

$\$ 10.60$

$\$ 13.60$ 
February 7, 1974

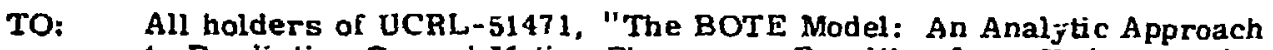
to Predicting Ground Motion Phenomena Resulting from Underground Nuclear Explosions"

FROM: Technical Information Department, L-3

\section{ERRATA}

Hease make the following corrections to your copy of UCRL-51471:

Page 2

Equation 4 should read:

$$
\Gamma=\Sigma_{0} \rho_{0} / \rho
$$

\section{Page 7}

In Table 1, the value for $h(f t)$ in the $\mathrm{BOTE}$ column should be: $\$ 00$

\section{Page 7}

Seventh line from the bottom should read:

"... normally done to look at the first few..."

\section{Page 8}

Fifth line frcm the bottom should read:

"... for tuffe of $100 \%$ and $50 \%$ water..."

\section{Page 9}

Third line of the caption for Fig. 8 should read:

"... for tufe having 100\%..."

Page 9

Third line of the caption for Fig. 9 should read:

"... for tuff having $50 \%$..." 
$-2-$

Page 17.

In Table A1, the value for $Y$ in the first set of units was omitted. It should be: $\mathrm{Y} \equiv \mathrm{kt}$

$\frac{\text { L. L. Linton }}{\text { Technical Information Department }}$ 
TID-4500, UC-11

Environmental and

Earth Sciences

\section{迎 \\ LAWRENCE UNERMOAE LABORATOFY \\ University of Calionia/Nivermara, Car $\mathrm{kmig} / 94550$}

UCRL-51471

\section{THE BOTE MODEL: ARS ANALYTIC APPROACH \\ TO PREDICTING GROUND MOTION PHENOMENA RESULTING FROM UNDERGROUND NUCLEAR EXPLOSIONS}

S. R. Kurtz

MS, date: November 13, 1973

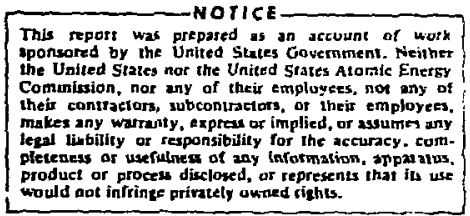




\section{Contents}

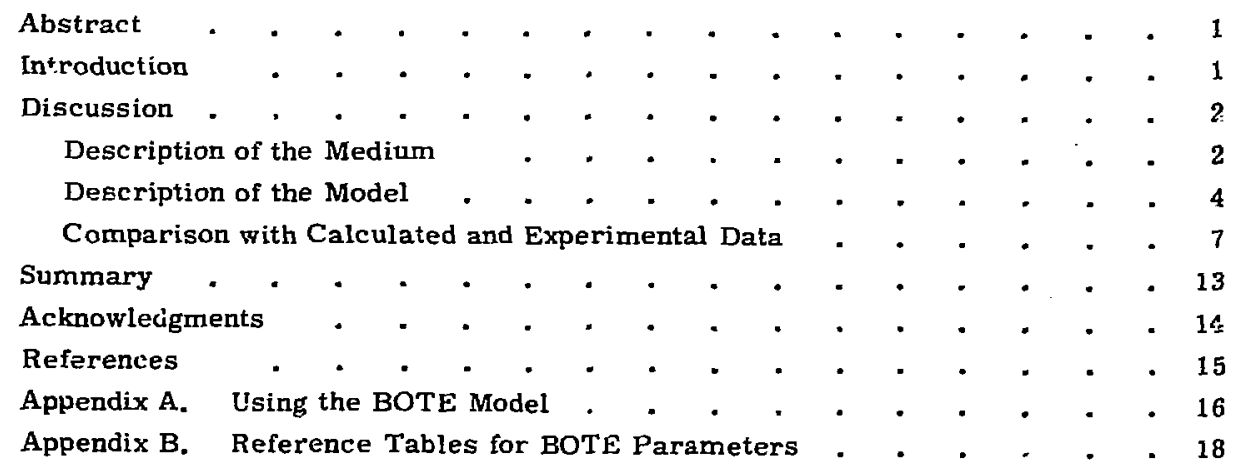




\title{
THE BOTE MODEL: AN ANALYTIC APPROACH TO PREDICTING GROUND MOTION PHENOMENA RESULTING FROM UNDERGROUND NUCLEAR EXPLOSIONS
}

\begin{abstract}
Astract
An analytic model (the BOTE model) based upon a superposition of the limiting forms for the outgoing stress wave (i.e., a strong shock at early times decaying to a simple acoustic wave at later times) is presented as a means to describe the ground-motion phenomena resulting from underground nuclear explosions. Taking

into account tho effects of both the porosity and the water content of the surrounding medium, the BOTE model provides good agreement with both calculn'ed and experimental data for times ranging from tens of microseconds to tens of millis:ccnds, and for distances ranging out to $350 \mathrm{ft} / \mathrm{kt}^{1 / 3}$.
\end{abstract}

\section{Introduction}

A great deal of time and effort is spent on attempis to predict the effects of underground nuclear explosions on the surrounding medium, with ground-motion phenomena often being of particular interest. Methods commonly used to predict these phenomena range from the use of sophisticated computer codes which use finite difference techniques to solve the hydrodynamic equations of motion, to the use of empirically derived scaling laws which relate one event with a particular set of parameters such as yield, and surrounding medium, to another event having a different set of parameters.

The primary difficulty in using computer codes lies in the amount of time and effort required to obtain results. Some codes (e.g. $\operatorname{soc}^{1,2,3}$ ) require a quite extensive knowledge of the equation of state, material properties, and geology of the surrounding medium, while other codes (e.g. RADOIL ${ }^{4}$ ) require many hours of computer time to obtain results out to only a few hundred microseconds past nuclear zero time.

On the other hand, the simple use of scaling laws to predict the results of a proposed event often leaves one with a rather uneasy feeling; for the coefficients anci exponents appearing in such laws are simply the results of empirical fits to experimenta! data. Although the similarities between these fits and the analytic solutions for a spherical blast wave have long been recognized, ${ }^{5,6}$ no firm basis in physical theory for these fits has been brought forth. Thus, one is left with the 
question as to whether physics or black magic is being used in making the predictions.

We present in this paper a simple analytic model (the BOTE ${ }^{*}$ model) which can be used to predict grounc motion phenomena. This model is based on a superposition of the limiting forms ior the outgoing stress wave (i.e., a strong shock at early times decaying to a simple acoustic wave at later times). While obviously being quicker and easier to use than a computer code, the BOTE model also has a more explicit basis in physical theory than do simple scaling laws.

\section{Discussion}

\section{DESCRIPTION OF TEE MEDIUM}

The first thing needed is a means of describing the medium in which the explosion is to take place. Because of the large effects of water content and total porosity upon the ground motion phenomena, 7,8 our description must take into account not only the medium type, but also its water content and total porosity.

Let us assume that we have an infinite homogeneous medium of initial density $\rho_{0}$; and that this medium can be tescribed by an equation of state of the form

$$
P=(\gamma-1) \rho L,
$$

where $P$ is the pressure, $\rho$ is the density, $I$ is the specific internal energy, and $\gamma$ is a dimensionless constant. Usage of this form of equation of state is felt to be justified by the very high pressures being considered. ${ }^{6}, 9$ Thus, except for the density $\rho$, no material properties, water content, or porosity are explicitiy taken into account. Rather, these omissions are taken into account implicitly by the value used for $\gamma$. In order to do this, we

\footnotetext{
"BOTE is an acronym for Back Of The Envelope.
}

need to relate the actual equation of state for the surrounding medium with our form shown in Eq. (1).

An equation of state commonly used to describe shocks in solids is the

Grüneisen equation of state

$$
P=\Gamma \rho\left(I-I_{L}\right)+P_{L},
$$

where $P$ is the pressure, $\Gamma$ is the Grüneisen gamma for solids, I is the specific internal energy, $I_{L}$ is the "lattice" internal energy due simply to interaction between neighboring particles, and $\mathrm{P}_{\mathrm{L}}$ is the "lattice" pressure. Because of the high pressures being considered when treating ground motion phenomena, the ${ }_{L}$ and $P_{L}$ terms become negligible and the Grüneisen equation of state becomes

$$
\mathrm{P} \cong \Gamma \rho \mathrm{I},
$$

which is of the same form as Eq. (1). Noting that

$$
\Gamma=\Gamma_{0} \rho / \rho_{0}
$$

where $\Gamma_{0}$ is the Grüneisen gamma at normal density $\rho_{0}$, we can rewrite Eq. (3) as

$$
\mathrm{P}=\Gamma_{0} \rho_{0} \mathrm{I} \text {. }
$$


For strong shocks, the limiting form for $\Gamma_{0}$ is

$$
\Gamma_{0}=\frac{2}{\frac{\rho}{\rho_{0}}-1} .
$$

From our assumed form of equation of state we know that

$$
\frac{\rho}{\rho_{0}}=\frac{\gamma+1}{\gamma-1}
$$

This equation when substituted into the expression for $\Gamma_{0}$ gives

$$
\gamma=\Gamma_{0}+1
$$

If we now assume that

$$
\Gamma_{0}=W \Gamma_{0 W}+(1-W) \Gamma_{0 R^{\prime}}
$$

where $W$ is the weight fraction of water, $\Gamma_{0 W}$ is the Grüneisen gamma for water, and $\Gamma_{0 R}$ is the Grüneisen ga mma for the porous rock, we get

$$
\gamma=W \Gamma_{0 W}+(1-W) \Gamma_{0 R}+1
$$

Schrceder and McMaster have shown that for water at $20^{\circ} \mathrm{C}$ the following expression can be used 10,11 :

$$
\Gamma_{\text {ow }}=2 S_{W}-2
$$

at

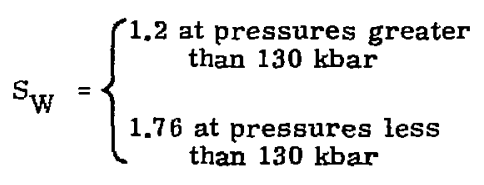

where $S_{W}$ is the slope of the shockvelocity, particle-velocity Hugoniot. The phase change of the water as it is shock vaporized is taken into account in Eq. (11).

From the McMaster-SchroederGrüneisen equation of state for solid porous material containing water ${ }^{11,12}$ we get
$\Gamma_{\mathrm{OR}}=\left[\frac{2 \mathrm{~S}_{\mathrm{R}}}{(1-W)} \frac{\rho_{\mathrm{g}}}{\rho_{0}}-1\right]\left[\frac{(1-W) \rho_{0}}{\rho_{\mathrm{g}}}\right]^{5}$,

where $W$ is the weight fraction of water. $\rho_{0}$ is the initial in situ density, $\rho_{\mathrm{g}}$ is the grain density, and $S_{R}$ is the slope of the shock-velocity, particle-velocity Hugoniot for the material when $\rho_{0}=\rho_{g}$ and $\mathrm{W}=0$. Schroeder and McMaster have shown $S_{R}$ to be related to the steepness of the atomic forces between atoms, 12 and as such, it is a measure of the compressibility of the material. An $S_{R}$ value of 1.3 can be used for both the tuff and alluvium found at the Nevada Test Site. ${ }^{11}$

Equation (12) can be rewritten to more clearly show its dependence on initial porosity if we first note that

$$
1-\phi-\frac{(1-W) \rho_{0}}{\rho_{g}} \text {, }
$$

where $\phi$ is the total porosity of the medium. Equation (12) then becomes

$$
\Gamma_{0 R}=\left[\frac{2 S_{R}}{1-\phi}-1\right][1-\phi]^{5} .
$$

Substituting Eq. (11) and eitser Eq. (12) or (14) into Eq. (10) results in the following expressions for $\gamma$ :

$$
\gamma=\left\{\begin{array}{c}
W\left[2 S_{W}-2\right]+(1-W) \\
\times\left[\frac{2 S_{R}}{(1-W)} \frac{\rho_{g}}{\rho_{0}}-1\right] \\
\times\left[\frac{(1-W)_{0}}{\rho_{g}}\right]^{5}+1 \\
W\left[2 S_{W}-2\right]+(1-W) \\
\quad \times\left[\frac{2 S_{R}}{(1-\phi)}-1\right][1-\phi]^{5}+1
\end{array}\right.
$$


DESCRIPTION OF THE MODEL

Let us assume that the nuclear explosion releases its total energy $Y$ in a short time interval at the center of a spherical explosion chamber of initial radius $R_{0}$. A shock wave which represents the ground shock will then propagate outward from this explosion point. We will consider the phenomena occurring after the ground shock has traveled through a distance $R_{s}$ which is large in comparison with $R_{0}$; thus the total mass of material set in motion by the explosion is large in comparison with the mass of the explosion products. This leads to the approximation that the energy release is instantaneous and occurs at a point source. The resemblance between early-time groundmotion phenomena and the self-similar solutions for a spherical-blast wave was recognized early in the history of underground testing; and, in fact, provided the basis from which the scaling laws were founded. However, the blast-wave solutions themselves were not used to predict ground-motion phenomena because a simple way to account for the properties of the surrounding medium did not exist. By using the method described in the preceding section this limitation is eliminated.

In a simple blast-wave model, we would restrict the distance that the ground shock has traveled from the explosion point by requiring that the pressure in the ground shock $P_{G}$ be sufficiently large so that the initial pressurs $P_{0}$ of the surrounding medium can be neglected. This is equivalent to neglecting the initialinternal energy $I_{0}$ and sound speed $C_{0}$ of the surrounding medium in comparison with the explosive yield $\mathrm{Y}$ and the shock velocity $V_{s}$, respectively. If we also assume that essentially all of the mass of the surrounding medium that has been swept out by the ground shock is contained within a thin layer of thickness $\Delta r$ immediately behind the leading surface of the shock front; then the outgoing shock wave can be characterized as a thin shell of high density material propagating outwardly from the explosion point at the shock velocity $\mathrm{V}_{\mathbf{s}}$.

If we define $\Psi$ as

$$
\Psi \equiv\left[\frac{3}{4 \pi} \frac{(\gamma-1)(\gamma+1)^{2}}{(3 \gamma-1)}\right]^{1 / 2}
$$

then the approximate solution according to Chernyi for a spherical blast wave gives $^{13}$ :

$$
\begin{aligned}
& R_{s}=R_{s 0}\left(\frac{Y}{\rho_{0}}\right)^{1 / 5} t^{2 / 5} \\
& V_{s}=\left\{\begin{array}{l}
v_{s 1}\left(\frac{Y}{\rho_{0}}\right)^{1 / 2} R_{s}^{-3 / 2} \\
v_{s 2}\left(\frac{Y}{\rho_{0}}\right)^{1 / 5} t^{-3 / 5}
\end{array}\right. \\
& P_{S}=\left\{\begin{array}{l}
P_{s 1}(Y) R_{S}^{-3} \\
P_{s 2}\left(p_{0}\right)^{3 / 5}(Y)^{2 / 5} t^{-6 / 5}
\end{array}\right.
\end{aligned}
$$

where

$$
\begin{aligned}
& R_{s 0} \equiv\left(\frac{5}{2}\right)^{2 / 5} \Psi^{2 / 5} \\
& v_{s 1} \equiv \Psi \\
& v_{s 2} \equiv\left(\frac{5}{2}\right)^{-3 / 5} \Psi^{2 / 5}
\end{aligned}
$$




$$
\begin{aligned}
& P_{s 1} \equiv\left(\frac{2}{\gamma+\mathrm{T}}\right) \Psi^{2} \\
& P_{s 2} \equiv\left(\frac{2}{\gamma+1}\right)\left(\frac{5}{2}\right)^{-6 / 5} \Psi^{4 / 5} .
\end{aligned}
$$

We note that we can derive from Egs. (17a,b, and c) the standard scaling laws for isopressure and isovelocity contours which show both position and time to scale like $(\mathrm{Y})^{1 / 3}$.

The interval of appîicability of a simple blast-wave model is rather limited, however. It is restricted initially by the requirement that $R_{B}$ be much greater than the initial radius of the explosion chamber. Although varying from shot to shot because of yield and initial geometry differences, this requirement is usually met by a few tens of microseconds. More importantly, the upper limit on the interval of applicability is restricted in two ways. Firat, extrapolation of this model past that point in time or space when the peak pressure has fallen off to a value where material properties become important is meaningless. Secondly, the theory behind this model no longer holds when $P_{\mathbf{g}}$ becomes of the order of $[(\gamma+1)]$ $(\gamma-1)] P_{0}$, where $P_{0}$ is the initial pressure of the surrounding medium. Based on empirical comparisons with experimental data, ${ }^{6}$ this upper limit is on the order of approximately $600 \mu \mathrm{sec} /(\mathrm{kt})^{1 / 3}$.

Although a simple blast-wave model is sufficient to describe the early time phenomenology while the outgoing stress wave can still be thought of as a strong shock, we also need to be able to describe those phenomena which occur during that time interval when the stress wave is intermediate between being a strong shock and being a simple acoustic wave. This interval corresponds to approximately $95 \%$ of the shock wave's lifetime, and is the interval during which we propose to use the BOTE model.

The BOTE model is based upon a superposition of the-limiting forms for the stress wave. We know that the stress wave decays from a strong shock wave moving with the shock velocity $V_{B}$ to a simple acoustic wave moving with the sound speed $C_{0}$. Because the velocity of the outgoing waye is given simply by the derivative with respect to time of the waye's position function, the above condition can be met by simply rewriting $\mathbf{R}_{g}$ and $v_{8}$ as

$$
\begin{aligned}
& R_{B} \equiv R_{B}+C t \\
& \psi_{B} \equiv y_{s}+C
\end{aligned}
$$

where $\mathbf{C}$ is either the elastic or bulk sound speed depending upon whether the stress wave is in the elastic or plastic pressure range.

We also know that the pressure in the stress wave cannot decay to a value less than the local overburden pressure $\mathbf{P}_{\mathbf{0}}$ This fact leads us to rewrite $P_{s}$ as

$$
\boldsymbol{F}_{\mathrm{s}} \equiv \mathrm{P}_{\mathrm{s}}+\boldsymbol{P}_{0^{\prime}}
$$

where

$$
P_{0}=P_{0} g\left(h-R_{s}\right) \text {. }
$$

with $g$ being the acceleration due to gravity $\left(5=9.81 \mathrm{~m} / \mathrm{s}^{2}\right)$, and $\mathrm{h}$ being the original depth below the earth's surface of the explosion point. Equation (20) is consistent with the form used in Eq. (19) to take into account the sound speed $C$. The 
spatial decay of $\sigma_{5}$ according to Eq. (20) will go predominantly as $\mathrm{R}_{s}^{-3}$; $\operatorname{sim}$ ilar to a simple blast wave. This is in agreetnent with the functional dependence seen In curves empirically fitted to the data. 6,13

The following Eq. (20) summarizes the BOTE model:

$A_{s}=R_{s 0}\left(\frac{Y}{p_{0}}\right)^{1 / 5} t^{2 / 5}+C t$

$r_{s}=\left\{\begin{array}{l}v_{s 1}\left(\frac{y}{\rho_{0}}\right)^{1 / 2} n_{s}^{-3 / 2}+c \\ v_{s 2}\left(\frac{y}{\rho_{0}}\right)^{1 / 5}+c\end{array}\right.$

$$
s_{s}=\left\{\begin{array}{l}
P_{s I}(Y) R_{s}^{-3}+P_{0} E\left(h-R_{s}\right) \\
P_{s 2^{1}\left(\rho_{0}\right)^{3 / 5}(Y)^{2 / 5} t^{-6 / 5}} \times\left[1+\frac{C}{R_{s 0}}\left(\frac{\rho_{0}}{Y}\right)^{1 / 5} t^{3 / 5}\right]^{-3} \\
+P_{0} B\left[h-R_{s 0}\left(\frac{Y}{\rho_{0}}\right)^{1 / 5} t^{2 / 5}-C t\right]
\end{array}\right.
$$

where $\mathrm{A}_{\mathrm{s0}}, \mathrm{v}_{\mathrm{s1}}, \mathrm{V}_{\mathrm{s2}}, \mathrm{P}_{\mathrm{sI}}$, and $\mathrm{P}_{\mathrm{s2}}$, are as defined in Eqs. (18a-e). At early times when the wave is still moving supersonicaliy (i.e., $\gamma_{\mathrm{s}}>\mathrm{C}$ ), the results of Eq. (19) reduce to those of Eq. (14).

Figures 1 through 3 compare the BOTE model with both a simple blastwave model and a simple acoustic-wave model for a 1-kt explosion in alluvium. Table 1 lists the input used in the three calculations.
Figure 1 compares ground shock position as a function of time as predicted by the three models. We see that the BOTE model differs from the simple blast-wave model by approximately $1.5 \mathrm{~m}$ at $1 \mathrm{~ms}$ and by approximately $15 \mathrm{~m}$ at $10 \mathrm{~ms}$.

Figure 2 compares ground-shosk velocity as a function of time for the three models. We see that the stresswave velocity according to the blast-wave model falls below the local sound speed at $1.5 \mathrm{~ms}$ while the velocity according to the BOTE model is within w10\% of the initial sound speed by $50.0 \mathrm{~ms}$.

Figure 3 compares the decay of the peak pressure in the ground shock as a function of range as predicted by the BOTE and blast-wave models. Also shown is the decay of overburden pressure as you approach the surface. We sce that the overburden pressure term in

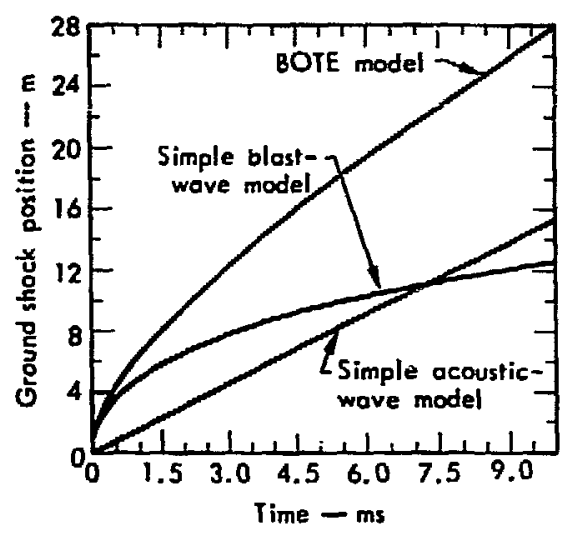

Fig. 1. A comparison of f $_{5}$ vs $t$ as predicted by: the BOTE model, a simple blast-wave model, and a simple acoustic model for a 1-kt explosion at a depth of 400 it in alluvium. The alluvium was assumed to have $\rho_{0}=2.0 \mathrm{~g} / \mathrm{cm}^{3}$, $\rho_{g}=2.65 \mathrm{~g} / \mathrm{cm}^{3}, \mathrm{~W}=0.005$, and

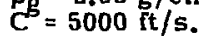




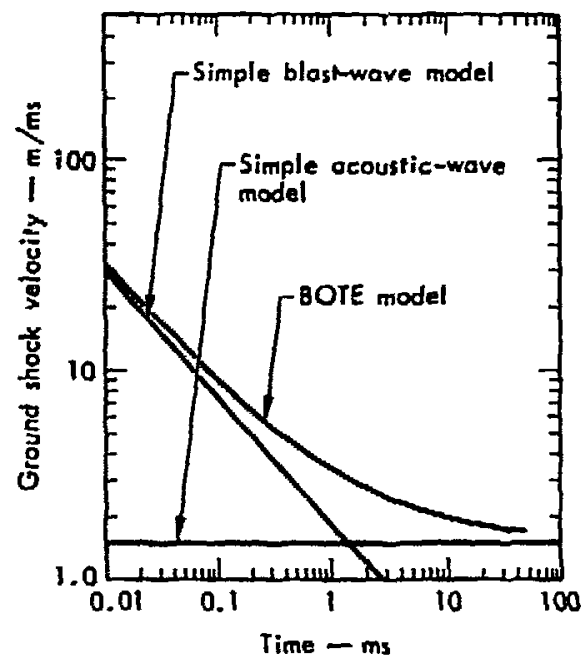

Fig. c. A comperison of $\nu_{5}$ va $t$ as predicted by: the BOTE model, a simple blast-wave model, and a simple acoustic model for the event described in Fig. 1.

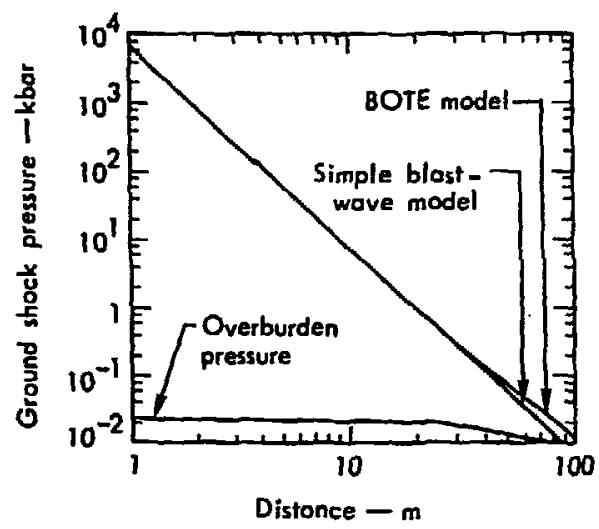

Fig. 3. A comparison of $\mathscr{S}_{\mathrm{s}}$ vs $\mathbb{R}$ as predicted by the BOTE model and a simple blast-wave model for the event described in Fig. 1. Also shown is the overburden pressure at each depth.
Table 1. Input used in comparing the BOTE, blast-wave, and acoustic models.

\begin{tabular}{lccc}
\hline & BOTE & $\begin{array}{c}\text { Blast } \\
\text { wave }\end{array}$ & Acoustic \\
\hline$Y(k t)$ & 1.0 & 1.0 & - \\
$S_{R}$ & 1.3 & 1.3 & - \\
$\rho_{0}\left(g / \mathrm{cm}^{3}\right)$ & 2.0 & 2.0 & - \\
$\rho_{g}\left(g / \mathrm{cm}^{3}\right)$ & 2.65 & 2.55 & - \\
$\mathrm{IV}$ & 0.05 & 0.05 & - \\
$C(f t / \mathrm{s})$ & 5000 & - & 5000 \\
$h(f t)$ & 1000 & - & 400 \\
\hline
\end{tabular}

the BOTE model does not become significant until distances greater than $30 \mathrm{~m}$ from the original working point are reached.

\section{COMPARISON WITH CALCULATED AND EXPERIMENTAL OATA}

The validity and usefulness of any model depends upon how well it agrees with experimental data. Thus, in order to demonstrate the validity of our assumptions, we now wish to compare results obtained with the BOTE model with both calculated and experimental groundmotion data.

Figures 4 through 6 show comparisons of results obtained from BOTE with a calculation done using the MEG code. ${ }^{14}$

MEG is a one-dimensional explosion code incorporating Lagrangian hydrodynamics and the calculation is typical of the type normally done to look at the first dew hundred microseconds after a nuclear explosion. The effects of a 100-kt explo.sion in tuff at a depth of burial of $1856 \mathrm{ft}$ were compared. The tuff was characterized by an initial density of $\rho_{0}=1.9 \mathrm{~g} / \mathrm{cm}^{3}$. a grain density of $\rho_{g}=2.43 \mathrm{~g} / \mathrm{cm}^{3}$, a 


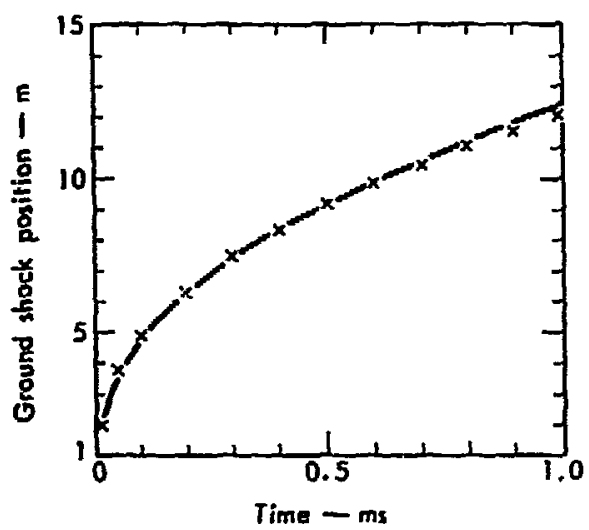

Fig. 4. A comparison of $A_{5}$ vs $t$ predicted by MEG $(x)$ and by the BOTE model (solid líne) for a 100-kt explosion at $1856 \mathrm{ft}$ in tuff. The tuff equation of state used in the MEG calculation was characterized by $\rho_{0}=1.9 \mathrm{~g} / \mathrm{cm}^{3}$, $\rho_{g}=2.43 \mathrm{~g} / \mathrm{cm}^{3}$. W $=0.17$, and $C^{g}=2500 \mathrm{ft} / \mathrm{s}$. Further comparisons with this calculation are made in Figs. 5 and 6.

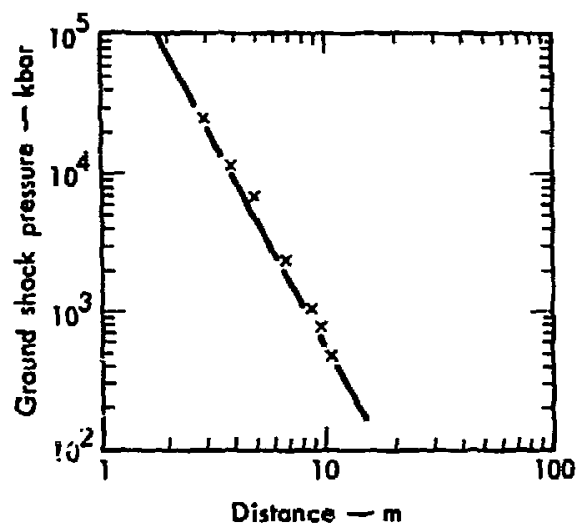

Fig. 5. A comparison of $S_{s}$ ve $t$ as predicted by MEG $(x)$ and by the BOTE model (solid line) for the event described in Fig. 4.

weight fraction of water of $W=0.17$, and a sound speed of $2500 \mathrm{ft} / \mathrm{sec}$. Using these values for $\rho_{0}, \rho_{\mathrm{g}^{2}}, \mathrm{~W}$, and $\mathrm{C}$ in the BOTE

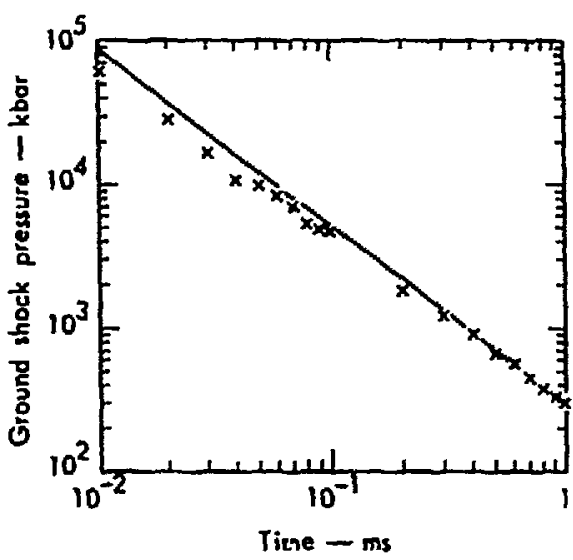

Fig. 6. A comparison of $S_{5}$ vs $\boldsymbol{R}$ as predicted by MEG $(x)$ and by the BOTE model (solid line) for the event described in Fig. 4.

model, we obtain results which agree quite well with the MEG data. Because MEG is an explosion code, and so calculates the exact solution for a spherical blast wave, the agreement seen in Figs. 4-6, although not perfect, indicates that the BOTE model does indeed reduce to a simple blast-wave solution at early times.

Figures 7 through 9 compare the results of the BOTE model with Butkovich's SOC calculations ${ }^{7}$ on the effect of saturation on ground motion phenomena. SOC calculations are typical of the type normaily done to determine the late-time phenomenology associated with the outgoing stress wave. Figure 7 shows pressure decay as a function of range for tuff having $0 \%$ water saturation. Figures $B$ and 9 are for tuffs of $50 \%$ and $100 \%$ water saturation, respectively. Table 2 lists the input used in the BOTE model in each case. Eiscept for $S_{R}$, all of the above data was taken from Butkovich's paper. ${ }^{7}$ 


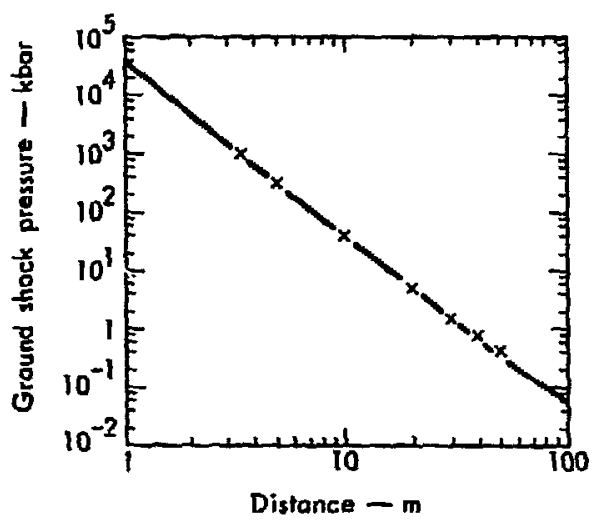

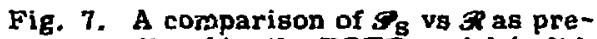
dicted by the BOTE model (solid line) and by Butkovich's SOC calculation (x) for tuff having $0 \%$ water saturation.

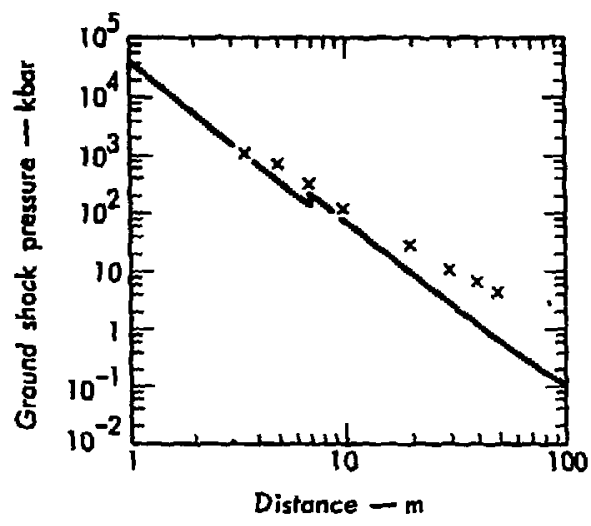

Fig. 8. A comparison of $\mathscr{S}_{\mathrm{g}}$ va $: \mathbb{R}$ as predicted by the BOTE model (Bolid line) and by Butkovich's SOC calculation ( $x$ ) for tuff having $50 \%$ water saturation.

The BOTE model and the SOC calculations agree reasonably well in each case, with quite good agreement for the $0 \%$ saturation case. The slight divergence between the results of the two methods as distance from the working point gets larger can be partially explained by the

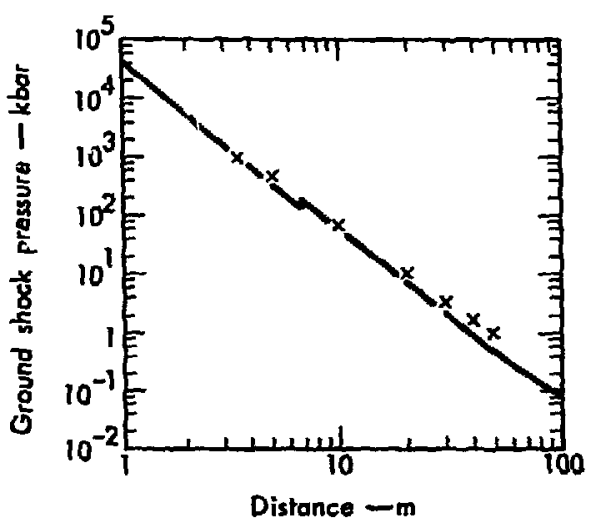

Fig. 9. A comparison of $S_{g}$ vs $R$ as predicted by the BOTE model (solid line) and by Butkovich's SOC calculation $(x)$ for tuff having $100 \%$ water saturation.

Table 2. Input used in comparing the BOTE model with Butkovich's SOC calculatione. 7

\begin{tabular}{llll}
\hline & $\begin{array}{c}0 \% \\
\text { saturation }\end{array}$ & $\begin{array}{c}50 \% \\
\text { saturation }\end{array}$ & $\begin{array}{c}100 \% \\
\text { saturation }\end{array}$ \\
\hline $\mathrm{S}_{\mathbf{R}}$ & 1.3 & 1.3 & 1.3 \\
$\mathrm{Y}(\mathrm{kt})$ & 10.0 & 10.0 & 10.0 \\
$\rho_{0}\left(\mathrm{~g} / \mathrm{cm}^{3}\right)$ & 1.40 & 1.605 & 1.81 \\
$\rho_{\mathrm{g}}\left(\mathrm{g} / \mathrm{cm}^{3}\right)$ & 2.37 & 2.37 & 2.37 \\
$\mathbf{W}$ & 0.005 & 0.127 & 0.226 \\
$\mathrm{C}(\mathrm{ft} / \mathrm{s})$ & 6700 & 6700 & 6700 \\
$\mathrm{~h}(\mathrm{ft})$ & 863 & 863 & 863 \\
\hline
\end{tabular}

fact that the BOTE model assumes an infinite medium, while the SOC calculations did not. Thus at large distances, the outgoing stress wave in SOC may be sensing the presence of the surface.

The discontinuous jump in the BOTE model's results for the $50 \%$ and $100 \%$ saturation cases at $P_{S}=130 \mathrm{kbar}$ is due to the water no longer being shock vaporized for pressures less than 130 kbar.

While comparisons with calculations are informative, the question of whether 
or not the comparison is being made with anything realistic can only be answered by comparison with experimental results.

A great deal of data giving ground shock position as a function of time is available. However, data concerning peak pressure in the outgoing stress wave is not so readily available. There is some peak pressure data available which can, and will, be compared to; however, the measurements usually made involve peak particle velocity as a function of range. This velocity is then related to the ground shock pressure by using the Hugoniot relationship

$$
P=\rho_{0} U V_{,}
$$

where $P$ is the ground shock pressure, $\rho_{0}$ is the initial in situ density, $V$ is the velocity of the outgoing stress wave, and $U$ is the peak particle velocity. Using the BOTE model's expression for $P$ and $V$ as functions of distance, we find that

$$
\mathrm{U}=\frac{P_{\mathrm{s} 1}(\mathrm{Y}) \mathrm{R}_{\mathrm{s}}^{-3}+\rho_{0} \mathrm{~g}\left(\mathrm{~h}-\mathrm{R}_{\mathrm{s}}\right)}{\rho_{0} \mathrm{~V}_{\mathrm{s} 1}\left(\frac{\mathrm{Y}}{\rho_{0}}\right)^{1 / 2} \mathrm{R}_{\mathrm{s}}^{-3 / 2}+\rho_{0} \mathrm{C}}
$$

In order to conveniently compare a large number of events, ve looked at data which had all been scaled to a yield of $1 \mathrm{kt}$. In order to use the BOTE model, we assumed realistic values for $\rho_{0}, \rho_{g}$, $W$, and $C$ which allowed the best agreement with the data. A scaled depth of

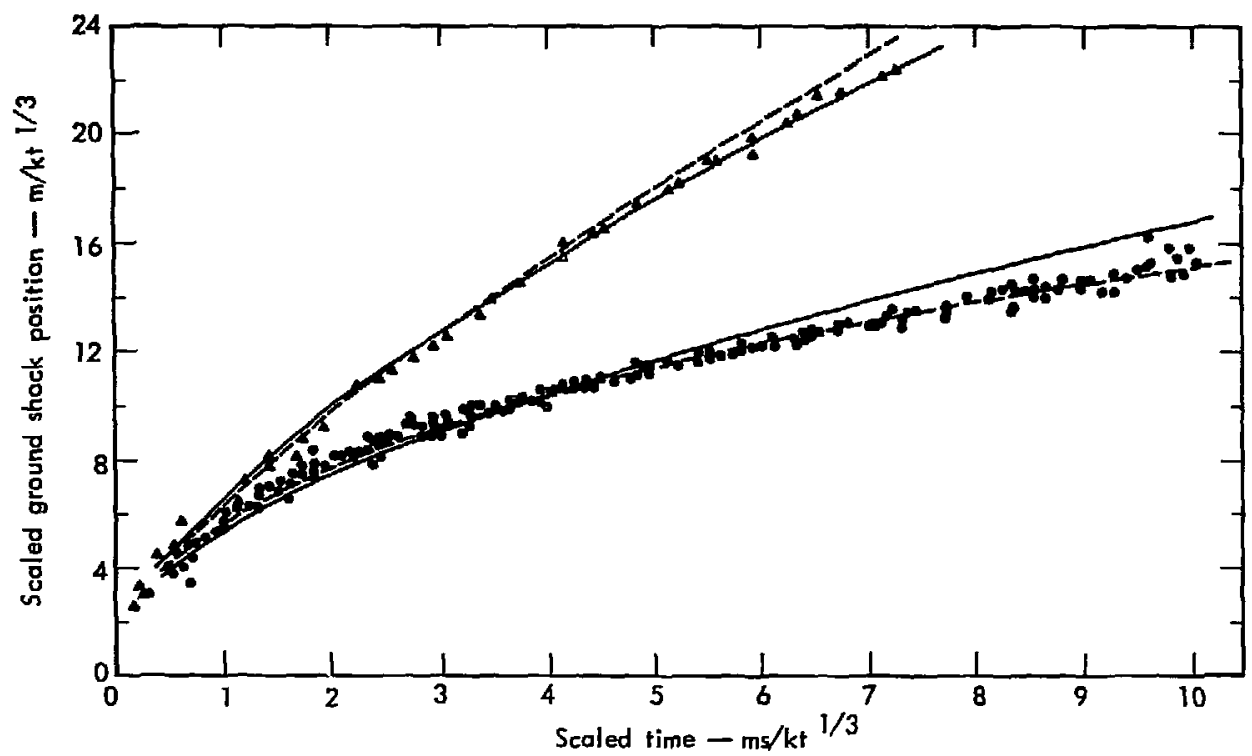

Fig. 10. A comparison of the BOTE model (solid line) with ground-shock position versus time data for both tuff and alluvium. The tuff data (triangles) comes from 3 events while the alluvium data (dots) comes from 19 events. All data has been scaled to a yield of $1 \mathrm{kt}$. SOC results (dashed line) are included for the sake of comparison. 
burial of $400 \mathrm{ft} / \mathrm{kt}^{1 / 3}$ was assumed in all cases. The somewhat arbitrary nature of the properties of our medium along with the fact that the characteristics of the tuff or alluvium in one area are not exactly the same as in another area explains the slight disagreement seen between some of the experimental data and our model.

Figure 10 shows ground shock pasition versus time data for both tuff and alluvium. ${ }^{2}$ Scaled results from 3 events

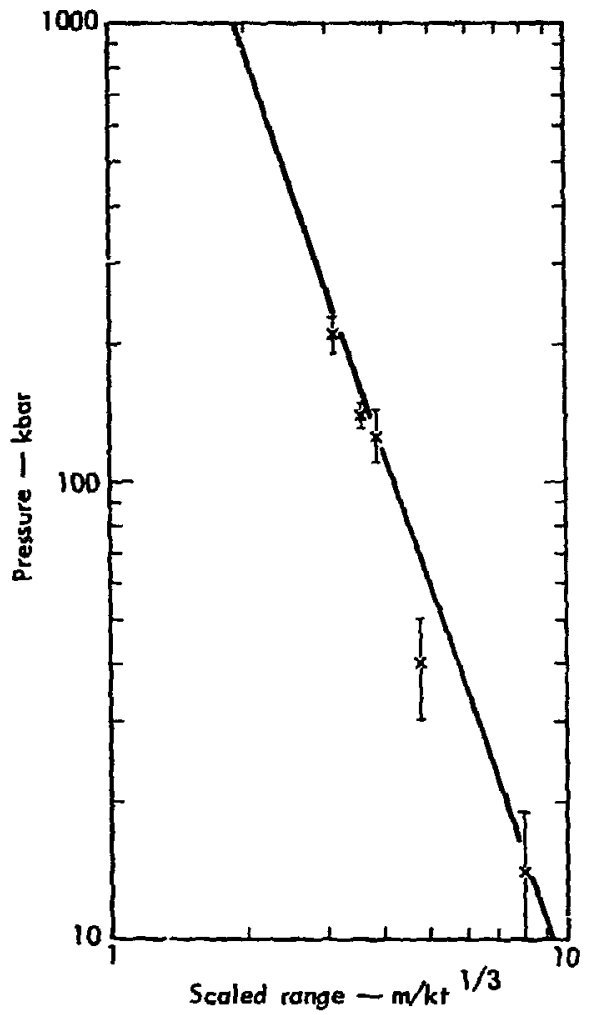

Fig. 11. A comparison of the BOTE model (solid line) with pressure data from five events executed in tuff. All data has been scaled to a yield of $1 \mathrm{kt}$. Error bars are indicated on the data. make up the tuff data, while scaled results from 19 events make up the alluvium data. In applying the BOTE model we assumed values listed in Table 3 to be representative of the medium.

Comparisons between the experimental data and our results are quite good over the time interval shown. The results of SOC calculations are also shown for the sake of comparison.

Figures 11 and 12 compare measurements of peak pressure in the ground

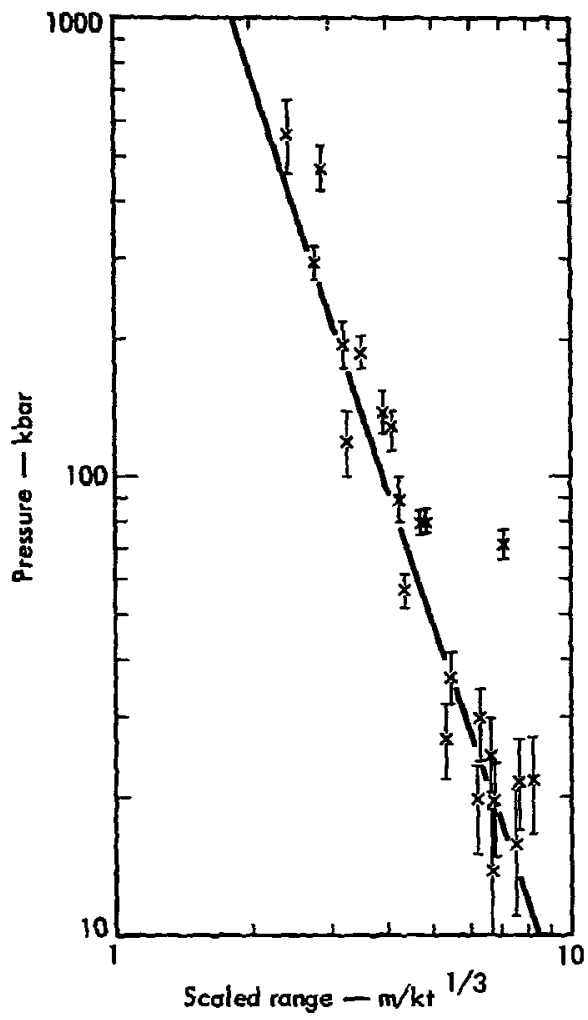

Fig. 12. A comparison of the BOTE model (solid line) with pressure data from 22 events executed in alluvium. All data bas been scaled to a yield of $1 \mathrm{kt}$. Error bars are indicated on the data. 
Table 3. Representative geologic data assumed when comparing the BOTE model with scaled ground-shock position data.

\begin{tabular}{cl}
\hline Tuff & Alluvium \\
\hline$\rho_{0}=1.9 \mathrm{~g} / \mathrm{cm}^{3}$ & $\rho_{0}=1.9 \mathrm{~g} / \mathrm{cm}^{3}$ \\
$\rho_{\mathrm{g}}=2.43 \mathrm{~g} / \mathrm{cm}^{3}$ & $\rho_{\mathrm{g}}=2.7 \mathrm{~g} / \mathrm{cm}^{3}$ \\
$\stackrel{W}{W}=0.01$ & $\mathrm{~W}=0.01$ \\
$\mathrm{C}=5000 \mathrm{ft} / \mathrm{s}$ & $\mathrm{C}=1500 \mathrm{ft} / \mathrm{s}^{2}$
\end{tabular}

The somew hat low value for $C$ indicates that the alluvium data was taken from shots near the sur'face.

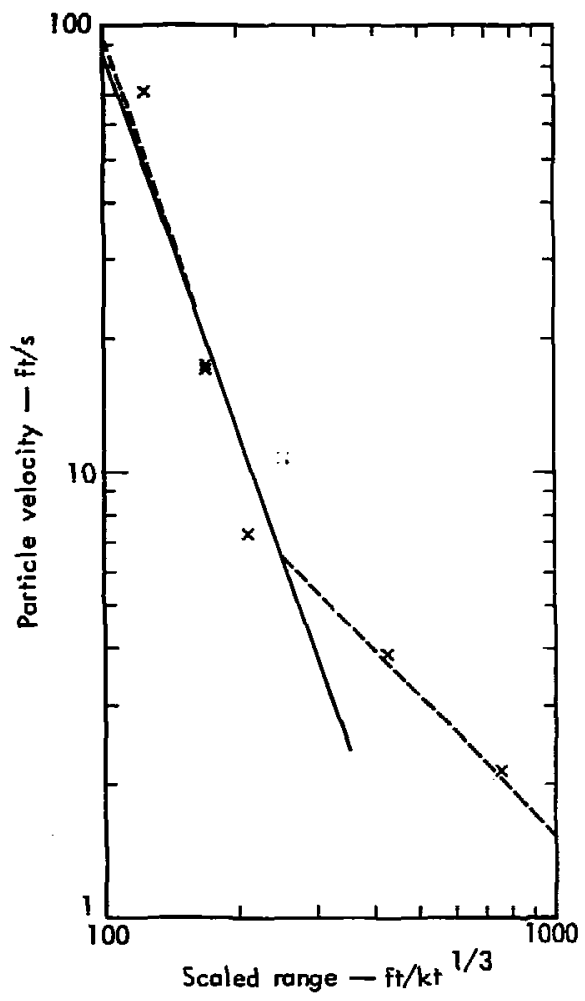

Fig. 13. A comparison of the BOTE model (solid line) with peak particle-velocity data for events executed in tuff. All data has been scaled to a yield of $1 \mathrm{kt}$. Perret's fit 16 (dashed line) to the data is also shown.
Table 4. Representative geologic data assumed when comparing the BOTE model with scaled ground-shock pressure data.

\begin{tabular}{ll}
\hline Tuff & \multicolumn{1}{c}{ Alluvium } \\
\hline$\rho_{0}=1.8 \mathrm{~g} / \mathrm{cm}^{3}$ & $\rho_{0}=1.9 \mathrm{~g} / \mathrm{ct}^{3}{ }^{3}$ \\
$\rho_{\mathrm{g}}=2.43 \mathrm{~g} / \mathrm{cm}^{3}$ & $\rho_{\mathrm{g}}=2.70 \mathrm{~g} / \mathrm{cm}^{3}$ \\
$\mathrm{~W}=0.01$ & $\mathrm{~W}=0.01$ \\
$\mathrm{C}=5000 \mathrm{ft} / \mathrm{s}$ & $\mathrm{C}=3000 \mathrm{ft} / \mathrm{s}$ \\
\hline
\end{tabular}

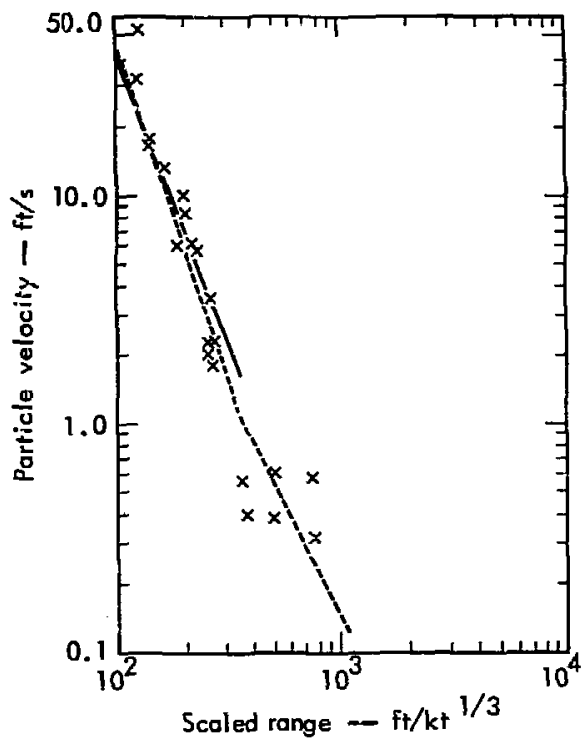

Fig. 14. Compares the BOTE model (solid line) with peak particlevelocity data for events executed in alluvium. AII data has been scaled to a yield of $1 \mathrm{kt}$. Also shown is a least square fit (dashed line) to the data.

shock with BOTE model results for tuff and alluvium respectively. ${ }^{6}$ The results of 5 events went into making up the tuff data, while 22 events make up the alluvium data. Table 4 lists the properties of the medium used in the BOTE model. 
The results of the BOTE model are seen to lie well within the scatter of the data in both figures.

Figures 13 and 14 compare peak particle velocity versus range data for unsaturated tuff and alluvium with the BOTE model. ${ }^{15}$ While the tuff data is all from one event, no indication of the number of events contributing to the alluvium data is available. The properties of the medium assumed in the BOTE model are lisied in Table 5. The BOTE model's results are seen to lie well within the scatter of the data in both cases. Also
Table 5. Representative geologic data assumed when comparing the BOTE model with scaled peakparticle-velocity data.

\begin{tabular}{cl}
\hline Tuff & Alluvium \\
\hline$\rho_{0}=1.95 \mathrm{~g} / \mathrm{cm}^{3}$ & $\rho_{0}=1.9 \mathrm{~g} / \mathrm{cm}^{3}$ \\
$\rho_{\mathrm{g}}=2.43 \mathrm{~g} / \mathrm{cm}^{3}$ & $\rho_{\mathrm{g}}=2.7 \mathrm{~g} / \mathrm{cm}^{\mathrm{J}}$ \\
$\mathrm{W}=0.005$ & $W^{\mathrm{W}}=0.01$ \\
$\mathrm{C}=5000 \mathrm{ft} / \mathrm{s}$ & $\mathrm{C}=3000 \mathrm{ft} / \mathrm{s}$ \\
\hline
\end{tabular}

shown are a curve derived by Perret ${ }^{16}$ for unsaturated tuff and a least squares fit to the alluvium data. ${ }^{17}$

\section{Summary}

In this paper, we have described a simple analytic model by which ground motion phenomena can be described for times ranging from tens of microseconds to tens of milliseconds after the detonation of an underground nuclear explosion. Using this model, we are able to predict the position of the stress wave as a function of time fairly accurately out to at least tens of milliseconds and the peak particle velocity (and so the peak pressure) in the wave as a function of range out to $350 \mathrm{ft} / \mathrm{kt}^{1 / 3}$.

However, like all models, the BOTE model has its limitations. At very early times, before the ground shock has had a chance to move far enough away from the explosion point so that its position $\Re_{\mathrm{S}}$ is large when compared to the initial radius $R_{0}$ of the explosion chamber, the results obtained from the BOTE model are meaningless. It usually takes a few tens of microseconds for the above condition to be met.

In comparing with SOC calculations, we note that the agreement between the BOTE model and the SOC results is better for lower values of water content W. This result is quite reasonable for the effect of water on ground motion phenomena is much more complicated than our simple linear combination of terms in Eq. (15) can account for. In spite of this limitation, however, we believe that the BOTE model's results are quite believable; for in all cases, the agreement with the SOC calculations is within acceptable bounás of uncertainty associated with such predictions.

Other limitations include the omission of precursor, layering and free surface effects, and the fact that the BOTE model is purely hydrodynamic in nature. 
However, the BOTE model as described in this paper is not intended as a replacement for either computer code calculations or scaling laws. Rather, it is hoped that this model will prove to be a useful supplement to these other methods.

\section{Acknowledgments}

We would like to thank Erlene Schooler for her aid in preparing the figures and tables included in this paper. We would also like to thank Ron Schroeder and
Gerry Nutt for their invaluable aid and discussions and Billy Hudson without whose support and encouragement this work could not have been done. 


\section{References}

1. J. T. Cherry and W. R. Hurdlow, "Numerical Simulation of Seismic Disturbances," Geophysics 31,33 (1966).

2. F. Holzer, Calculation of Seismic Source Mechanisms, Lawrence Livermore Laboratory, Rept. UCRL-12219 (1965).

3. J. F. Schatz, The Physics of SOC and TENSOR, Lawrence Livermore Laboratory, Rept. UCRL-51353 (1973).

4. W. Johnson, RADOIL and RAM, System, Science, and Software, La Jolla, California, Rept. 3SR-104 (1971).

5. J. Rambo, Lawrence Livermore Laboratory, private communication.

6. J. P. Mutachlecner and B. R. Saunders, Hydrodynamic Measurements of Yields of Underground Tests Durin-Niblich, Wehtstone, and Flintlock, Los Alamos Scientific Laboratory, Rept. LA-3971 (1968) (SRD).

7. T. R. Butkovich, Effects of Water Saturation on Underground Nuclear Detonations, Lawrence Livermore Laboratory, Rept. UCRL-51110 (1971).

8. B. K. C rowley, Effects of Porosity and Saturation on Shock Wave Response in Tuffs, Lawrence Livermore Laboratory, Rept. UCRL-74207 (1972).

9. W. J. Rae, "Impact Generated Shock Propagation," in High Velocity Impact Phenomena, R. Kinslow, Ed. (Academic Press, New York, 1970).

10. R. C. Schroeder and W. H. McMaster, "Shock-Compression Freezing and Melting of Water and Ice," J. Appl. Phys. 44, 2591 (1973).

11. R. C. Schroeder, Lawrence Livermore Laboratory, private communication, to be published.

12. R. C. Schroeder and W. H. McMaster, Compilation of Shock Hugoniot Data and Hugoniot Melting Points for the Elements, Lawrence Livermore Laboratory, Rept. UCRL-51253 (1972).

13. Ya. B. Zel'dovich and Yu. P. Raizer, Physics of Shock Waves and High Temperature Hydrodynamic Phenomena (Academic Press, New York, 1966), Vol. 1.

14. H. Smith, MEG Equations, Lawrence Livermore Laboratory, Rept. UCID-15366 (1967) (title $U$, report SRD).

15. V. E. Wheeler and R. G. Prestan, Scaled Free-Field Particle Motions from Underground Nuclear Explosions, Lawrence Livermore Laboratory, Rept. UCRL50563 (1968).

16. W. R. Perret, Subsurface Motion from a Confined Underground Detonation Part I, Operation Plumbbob, Sandia Laboratories, Albuquerque, Rept. WT-1529 (1961).

17. B. C. Hudson, Law rence Livermore Laboratory, private communication. 


\section{Appendix A \\ Using the BOTE Model}

When using the BOTE model, extreme care must be taken with respect to units. For instance, if one indiscriminately uses units of $Y \equiv \mathrm{kt}, \rho_{0} \equiv \mathrm{g} / \mathrm{cm}^{3}, \mathrm{~g} \equiv \mathrm{m} / \mathrm{s}^{2}$, $\mathrm{h} \equiv \mathrm{m}, \mathrm{R}_{\mathrm{s}} \equiv \mathrm{m}$ in the equation for $\mathscr{P}_{\mathrm{s}}$ as a function of distance, you would end up with $\mathscr{P}_{\mathrm{S}}$ being expressed in units of

$$
P_{s} \equiv \frac{\mathrm{kt}}{\mathrm{m}^{3}}+\frac{\mathrm{g} \cdot \mathrm{m}^{2}}{\mathrm{~cm}^{3} \cdot \mathrm{s}^{2}}
$$

This is not an exceptionally useful unit for the expression of pressure. Similar problems with ridiculous output units arise in using the other equations as well.

With the above difficulty in mind, we set about to determine conversion coefficients that would enable is to get results in reasonable units from input also in reasonable units. We first rewrote the defining equations for the BOTE model as:

$$
\begin{aligned}
& \mathscr{R}_{s}=R_{s 0}\left(\frac{\alpha Y}{\rho_{0}}\right)^{1 / 5} t^{2 / 5}+C t \\
& V_{s}=\left\{\begin{array}{l}
V_{s 1}\left(\frac{\alpha Y}{\rho_{0}}\right)^{1 / 2} R_{s}^{-3 / 2}+C \\
V_{s 2}\left(\frac{\alpha Y}{\rho_{0}}\right)^{1 / 5} t^{-3 / 5}+C
\end{array}\right. \\
& \mathscr{P}_{\mathrm{s}}=\left\{\begin{aligned}
\mathrm{P}_{\mathrm{s} 1}(\alpha \mathrm{Y}) \mathrm{R}_{\mathrm{s}}^{-3}+\beta \rho_{0} \mathrm{~g}\left(\mathrm{~h}-\mathrm{R}_{\mathrm{s}}\right) & \mathrm{P}_{\mathrm{s} 2}\left(\rho_{0}\right)^{3 / 5}(\alpha \mathrm{Y})^{2 / 5} \mathrm{t}^{-6 / 5}\left[1+\frac{\mathrm{C}}{\mathrm{R}_{\mathrm{s} 0}}\left(\frac{\rho_{0}}{\alpha \mathrm{Y}}\right)^{1 / 5} \mathrm{t}^{3 / 5}\right]^{-3} \\
& +\beta \rho_{0} \mathrm{~g}\left[\mathrm{~h}-\mathrm{R}_{\mathrm{s}}\left(\frac{\alpha \mathrm{Y}}{\rho_{0}}\right)^{2 / 5}-\mathrm{Ct}\right]
\end{aligned}\right. \\
& \mathrm{U}=\frac{\mathrm{P}_{\mathrm{s} 1}(\alpha \mathrm{Y}) \mathrm{R}_{\mathrm{s}}^{-3}+\beta p_{0} \mathrm{~g}\left(\mathrm{~h}-\mathrm{R}_{\mathrm{s}}\right)}{\rho_{0} \mathrm{~V}_{\mathrm{sl}}\left(\frac{\alpha \mathrm{Y}}{\rho_{0}}\right)^{1 / 2} \mathrm{R}_{\mathrm{S}}^{-3 / 2}+\rho_{0} \mathrm{C}}
\end{aligned}
$$

where $\alpha$ and $\beta$ are the conversion coefficients. Table A1 lists values of $\alpha$ and $\beta$ for two particular sets of units. 
Table A1. Magnitudes of the conversion coefficients $a$ and $\beta$.

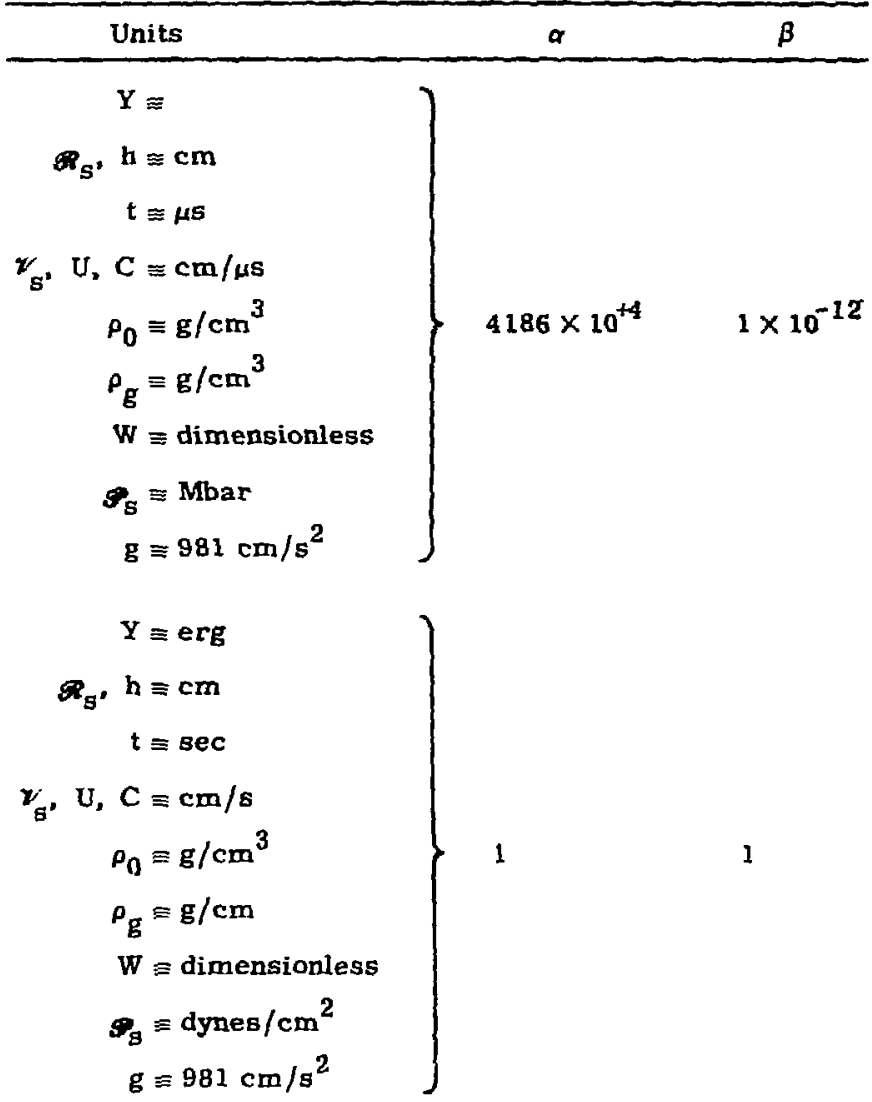

While the above systems of units are perhaps not the most convenient ones in all cases, they do have the advantage that $\alpha$ and $\beta$ have the same values in all of the equations that they appear in. 


\section{Appendix B \\ Reference Tables for BOTE Parameters}

The following tables are included in order to facilitate the use of the BOTE model. Tables $B 1$ and $B 2$ list values of $\gamma$ for various combinations of in situ density ( $\rho_{0} \equiv R H O$ ), grain density ( $\rho_{g} \equiv$ RHOG), and weight fraction of water (W). Table B1 would be used for peak pressures greater than $130 \mathrm{kbar}$, while Table $\mathrm{B2}$ would be used for pressures less than 130 kbar. Tables B3 and B4 list values of $\gamma$ for various combinations of weight fractions of water $(W)$ and total porosity ( $\phi \equiv P H I)$. Table $B 3$ is for pressures greater than 130 kbar, while Table B4 is for pressures less than 130 kbar.

Table B5 lists values of $R_{s 0}, V_{s 1}, V_{s 2}, P_{s 1}$, and $P_{s 2}$ for various values of $\gamma$. Figures $\mathrm{B} 1$ to $\mathrm{B} 5$ are plots of the values listed in Table $\mathrm{B} 5$ and are included to aid in interpolation between the values listed in Table B5. 

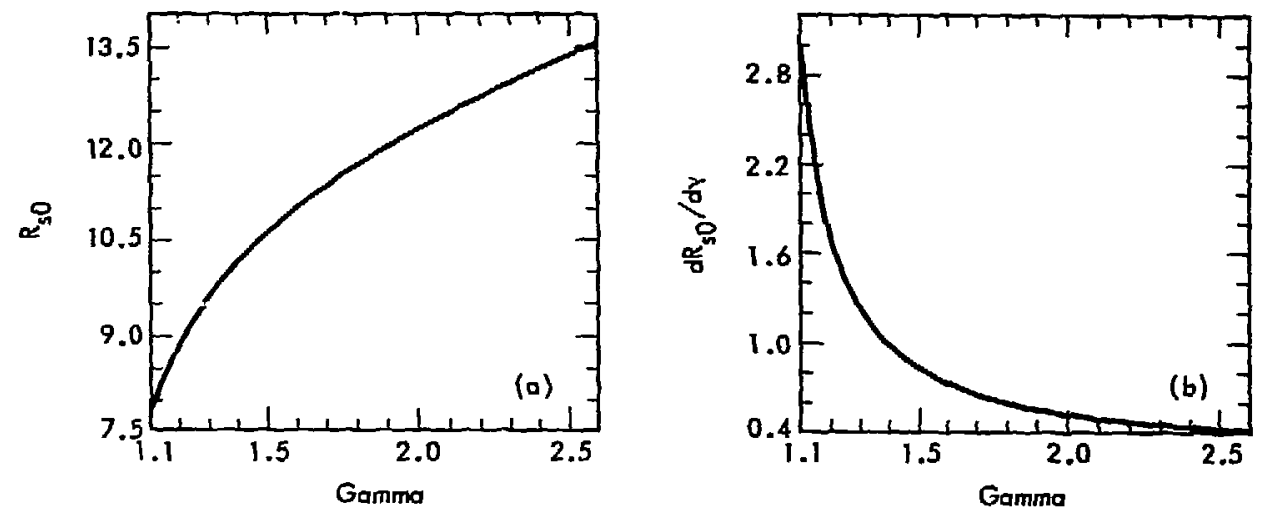
Fig. B1. Plot (a) shows $R_{\mathrm{s0}}$ as a function of $\gamma$, while plot (b) shows $\mathrm{dR}_{\mathrm{s0}} / \mathrm{d} \gamma$ as a
function of $\gamma$.
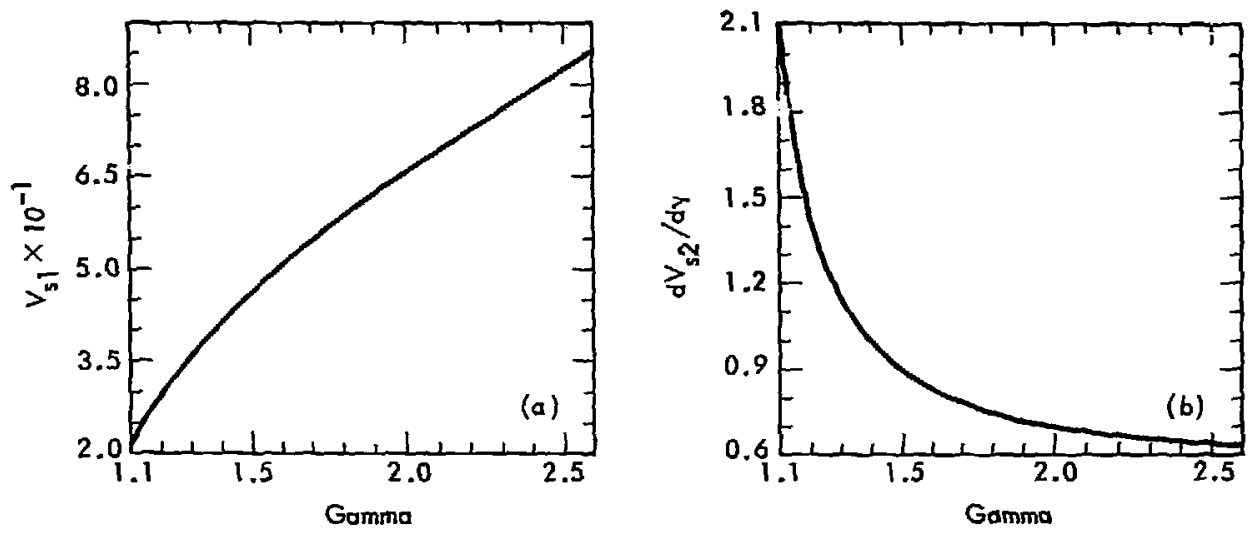

Fig. B2. Plot (a) shows $\mathrm{v}_{\mathrm{sI}}$ as a function of $\gamma$, while plot (b) shows $d \mathrm{v}_{\mathrm{sI}} / \mathrm{d} \gamma$ as a
function of $\gamma$. 

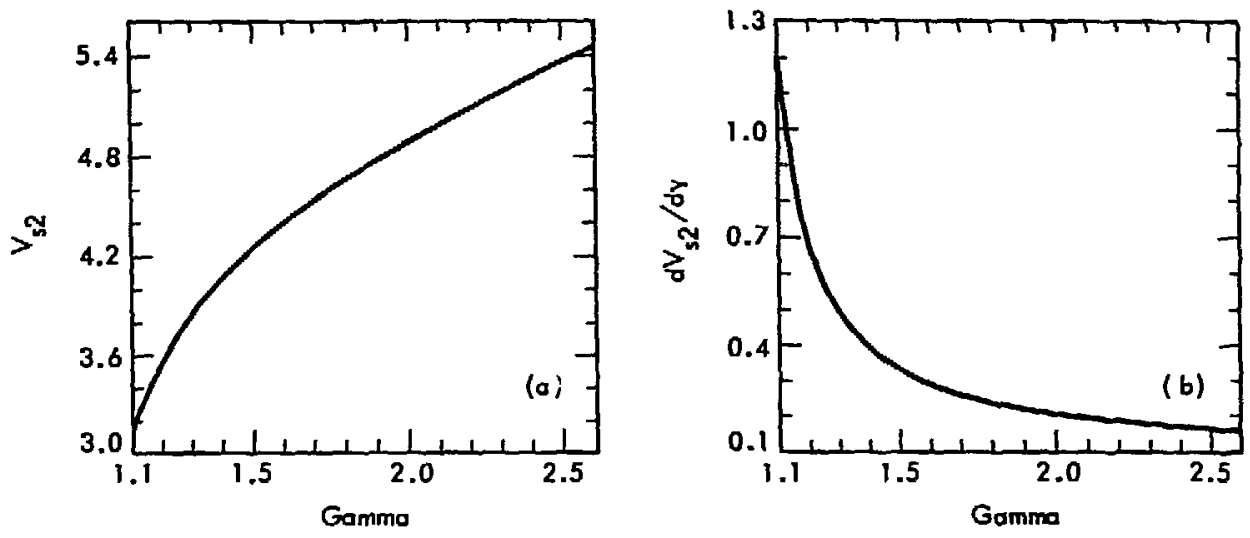

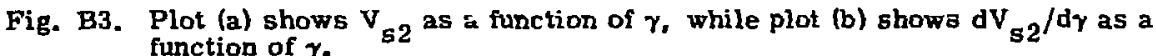
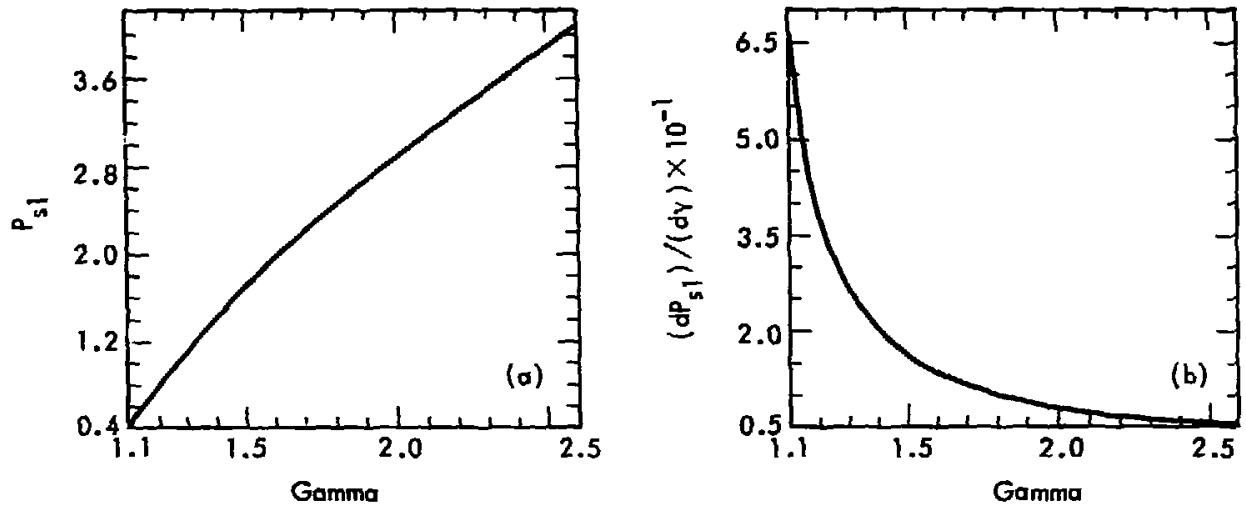
Fig. B4. Plot (a) shows $P_{s 1}$ as a function of $\gamma_{2}$ while plot $(b)$ shows $d P P_{s 1} / d \gamma$ as a
function of $\gamma$. 

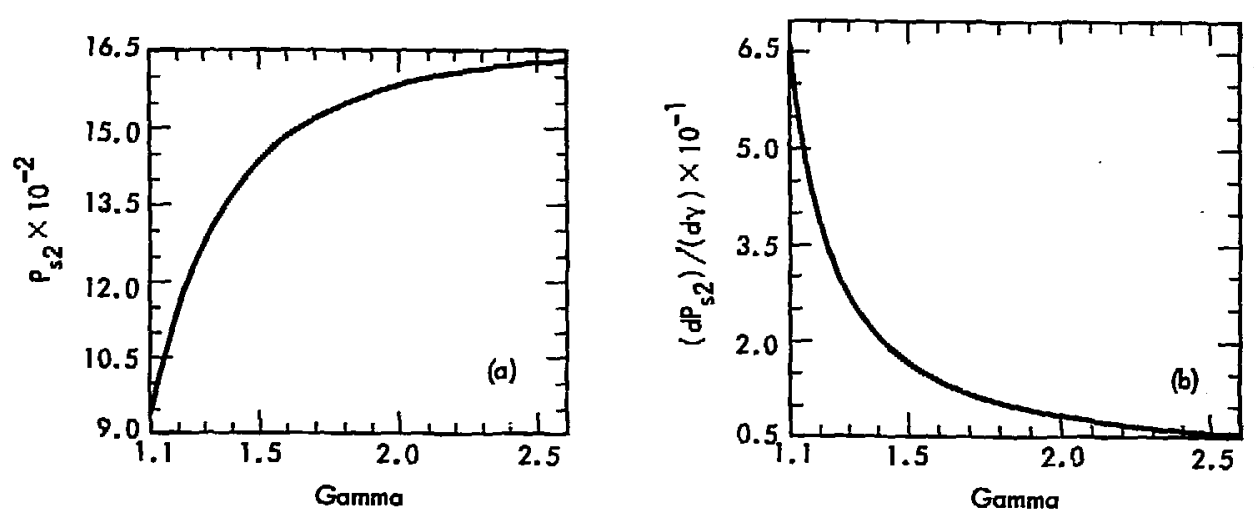

Fig. B5. Plot (a) shows $P_{s 2}$ as a function of $\gamma$, while plot $(b)$ shows $\mathrm{dP}_{\mathrm{s} 2} / \mathrm{d} \gamma$ as a
function of $\gamma$. 

Table B1. Values of $\gamma$ for various combinations of in pitu density ( $\rho_{0}$ aHO), grain density $\left(\rho_{\mathrm{g}} \equiv \mathrm{RFOG}\right.$ ), and welght fraction of water (W).
These values would be used when $\Phi_{\mathrm{g}}>130 \mathrm{kbar}$.

GAMMA $=1+(1-h)(12 S H /(1-h))(H+C G / R H C)-1)(11-h) H H C / R R[E) * D+W(2 S H-2)$

$$
5 H=1.3 \mathrm{C} \quad \mathrm{NHCG}=2.20 \quad 5 \mathrm{~K}=1.20
$$
\%

$1.00000 t-02+1.091<8 E+00$ $2.00000 t-02+1.09114 E+00$ $3.0000 U E-C 2$ 1.09115E+00 4.00000E-02 $+1.09131 t+00$ $5.00000 t-02+1.09162 t+00$ $6.00000 t-02+1.09207 E+00$ T.0000 $9.00000 t-02 * 1.094<4+00$ $1.000001-01+1.094245+00$ 1.000001 .01 . $1.095235+00$

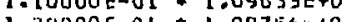
1.20000

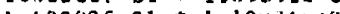
1.400001 .01 1.10u.36L

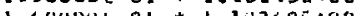
1.hoovot-ol a 1.10360e+00 $1.70000 t-01 * 1.105385+00$ $1 . .00000 L-01$ 1.10725E+00 $1.90000[-01 * 1.10922 k+0$ $2.00000 t-01 * 1.11128 t+00$ $2.10000 t-01+1.11344 t+00$ $2.20000 t-01$ 1.115t7t+0 $2.30000 t-01$ \$.116cot +00 $2.40000 t-01 \% 1.12040 t+00$ $2.50000 t-01$ 1.122e8rtou $2.60000 t-01 * 1.125445+00$

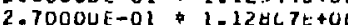
$2.80000 \mathrm{~F}-01$ 1.13L17L+00 $2.900001-01$ 1.13 $34 t^{2}+00$ $3.00000 t-01$ 1. $13637 t^{2} 00$ 3.00000 ol 1.1363 tetao $3.10000 t-01$ 1.13427t+u 3.20000 tol $1.142<26+03$ $3.30000 \mathrm{r}$ * $1.145 \mathrm{st}+00$ $3.40000 \mathrm{r}-01+1.14030 t+0$ $3.50000 t-01 \% 1.1514 L_{2}+00$ $3.60000+01 * 1.154 \mathrm{jul}+00$

\begin{tabular}{|c|c|}
\hline 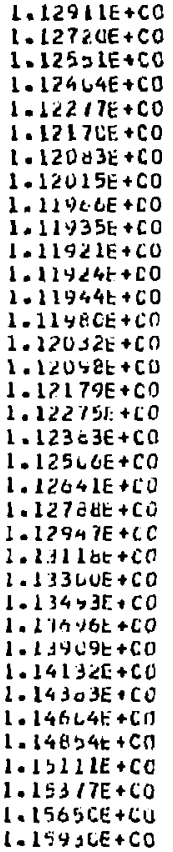 & 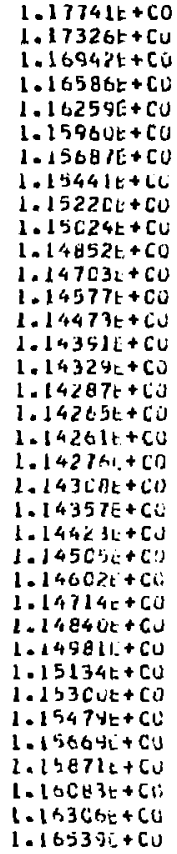 \\
\hline
\end{tabular}

$\begin{array}{ll}1.12911 E+C O & 1.17741 t+C O \\ 1.12720 E+C O & 1.17326 t+C O\end{array}$ $1.125 \supset 1 E+C O \quad 1.16947 t+C$ $1217 E+C O \quad 1.16259 E+C$ 1217CE +CO 1.1596Ut+CO 1.15687E+co $1.14473 t+C$ $1.14324 L+C$ $1.14265 t+C u$ HIE + C $145 C^{j} c+C$ $.14602 t+C i$ -14714E+CO $1.149811+\mathrm{Cu}$ 3 Cut $+C 0$ $1.15474 t+C$ 2. 1 เE $1 t+C$ $1.16539 i+C u$

\section{$1.237635+00$} $.230726+00$ $1.22421 E+00$ $1.21233 t+00$ $1.20644 E+00$ 1. $20192 E+i$ 1. $197245+10$ 1. $19724 E+10$ 1. $142.0 \mathrm{E}+0$ 1. $18521 t+C 0$ 1. IRI $33 E+0$ 1.1787OE+O 1. $175 \times 8 E+U 0$ $1.13348+00$ $1.17120: 00$ $1.16431 t+00$ $1.16762 E+U$ 1. $16618 t+00$ 1. $16497 t+U 0$ $1.264 J 3 E+00$ I. $1 E 33 \cup E+O U$ I. 1 AL TYE +US $1.16<44 E+60$ Le 1. 1. 1 L251Et00 1. $18281 \mathrm{C}+00$ $1.16130 E+00$ 1. $16.376 t+00$ $1658 u t+00$ 1.16580 tud 1. $16097 \mathrm{E}+0$ $1.16820 t+u 0$ $1.16974 t+00$ $1.17135 t+0 u$
$1.1730 t+00$

\section{$1.31123 E+00$ \\ 1. 3. $462 E+00$} 1. $30047 \mathrm{E}+\mathrm{CO}$ $1.29122 E+L O$ $1.28197 t+00$ $1.27321 t+00$ $1-26443 E+40$ $1.25711 \mathrm{~L}+4 \mathrm{C}$ $1.24414 t+00$ $1.24<81 t+00$ $1.23631 t+00$ $1.234555+00$ $1.21+265+00$ 1. $14305+40$ $1.214366+00$ $1=2 u+83 t+00$ $1.20>06 t+00$ . $26183 t+00$ 1. 1 th $3 \mathrm{E}_{\mathrm{t}+0 \mathrm{U}}$ $1.143141:+00$ $1.14236 t+00$ - I By $83 t+40$ Id ItUOE +UU $1.1856 i 6 t+u 0$ $1.104 \mathrm{COL}+U \mathrm{O}$ $1.1+2 t 1 t+00$ $1.10148 t+10$ $1448 E+00$ I. Iyubtito $1.11946 t+00$ $1.11430 z+00$ $1.17 \times 42 t+40$ $1.17+67 L+60$ $1.210<3 E+0$ $1 . I$ IU $77_{L}+00$ 1. 1 alo01.t00 i. Iy $261 t+00$ I. $38537 \mathrm{E}+\mathrm{CO}$ 1. $3717 \mathrm{TE}+\mathrm{CO}$ 1. $358815+00$ 1. $34646 \mathrm{E}+\mathrm{DU}$ $1.13472 t+C u$ $1.12356 E+C U$ 1. $31294 r+00$ 1 3020 0 1.2.) $34 \mathrm{FE}+\mathrm{CO}$ $1=24453 t+00$ - 28 a 31 + 1. $2610165+01$ 1.20L711.t2 $1.2472 \mathrm{se}+\mathrm{CO}$ I. $2411 S E+C D$ 1 - $23352 \pi+\mathrm{C} 0$ $1.21 C 3 U E+C O$ $1.27>44 t+C 0$ 1.221UEE+CO 1. $2110+\mathrm{E}+\mathrm{CO}$ $1-2132 A r+\Delta U$ 2- $\angle 1001 t+C C$ $1.20311 \mathrm{t}+\mathrm{Cr}$ 2. $2 U 449 E+C O$ $1.204495+C 0$
$1.202101+C 0$ 1. $200201+00$ $1.23450 \mathrm{C}+\mathrm{CC}$ 1. lit?

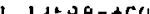
. $11313:+C 0$ l. $11453 E+C C$ d. $144190+C 6$ l. $1740 \mathrm{Ht}+6 \mathrm{O}$ $1.1+421++01$

$1.50000 E+C O \quad 1.6 U C O C E+00$

1. 7 COCOE+ DO

\begin{tabular}{|c|c|}
\hline 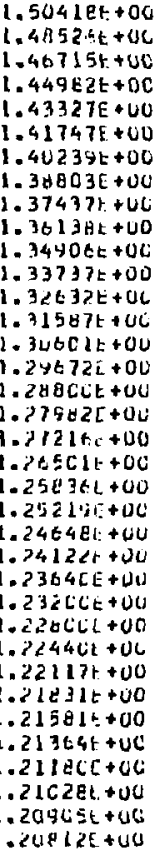 & $\begin{array}{l}1.62 G 1 B E+00 \\
1.6 C 19 B E+00 \\
1.57855 E+00 \\
1.55619 E+00 \\
1.53477 E+00 \\
1.51426 E+00 \\
1.49465 E+00 \\
1.47591 E+00 \\
1.45802 E+00 \\
1.44097 E+0 C \\
1.42472 E+00 \\
1.4 C 926 E+00 \\
1.34457 E+0 C \\
1.34062 E+00 \\
1.36711 E+00 \\
1.15490 E+00 \\
1.34307 E+00 \\
1.33142 E+00 \\
1.32141 E+00 \\
1.31154 E+00 \\
1.36227 E+00 \\
1.29360 E+00 \\
1.22354 E+00 \\
1.27745 E+00 \\
1.27 C 95 E+00 \\
1.26446 E+00 \\
1.25848 E+00 \\
1.25298 E+00 \\
1.24746 E+00 \\
1.24339 E+00 \\
1.23925 E+00 \\
1.2355 E+00 \\
1.23223 E+00 \\
1.22932 E+00 \\
1.22618 E+00 \\
1.22460 E+00\end{array}$ \\
\hline
\end{tabular}


GAMMA $=1+(1-W)((2 S K)(1-h))(R H C U / R H C)-1)(1)-W) H H C / R+C G)+A]+k(2 S W-?)$

$$
S R=1.3 \mathrm{C} \quad H H L G=2.20 \quad S_{W}=1.20
$$

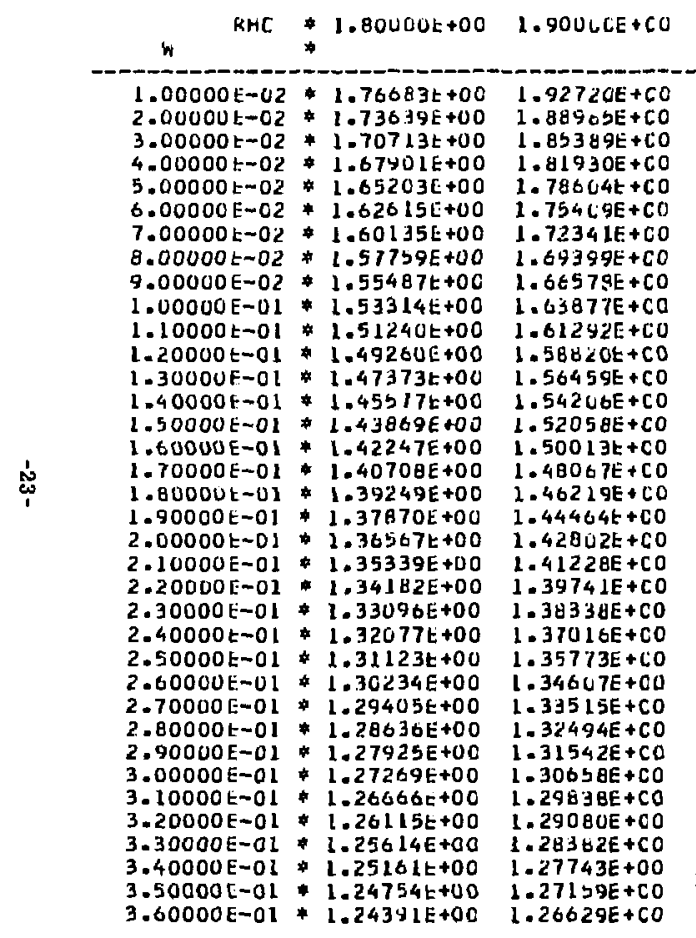

$2.208225+100$ $2.66318 t+[u$ 2. $0197 h t+C 0$ $1.97794 t+C O$
$1.93767 t+C O$ $1.89894 t+C O$ $1.86170 t+C u$ $1.02592 t+C O$ $1.7915 \Omega E+C U$ $1.75863 \mathrm{E}+\mathrm{CO}$ $1.72705 i+C O$ $1.727 \mathrm{CL}+\mathrm{C}$ $1.61381 \mathrm{t}+\mathrm{c}$ $1.66787 \mathrm{E}+\mathrm{CO}$ $1.64 C 20 E+C O$ $1.61377 \mathrm{~L}+\mathrm{CO}$ 1.5eBsye + Co 1. $56450 \mathrm{t}+\mathrm{CO}$ $1.54161 \mathrm{E}+\mathrm{CO}$ $1.51983 t+C 0$ $1.49913 E+C 0$ $1-47950 E+C U$ $1.44327 t+C O$ $1.4266 \% E+C O$ $1.41090 E+C U$ 1. $39610 \mathrm{OC}+\mathrm{C}$ 1. $39610 L+C$ $1.3821 \mathrm{BL}+\mathrm{CO}$ $1.36911 E+C 0$ 1. $34541 E+C O$ $1.34541 E+C$ 1. $33474 E+C O$ 1. $32481 E+C O$ 1. $31559 E+C$ $1.30707 E+C 0$ $1.29201 E+C U$

\begin{tabular}{|c|c|}
\hline 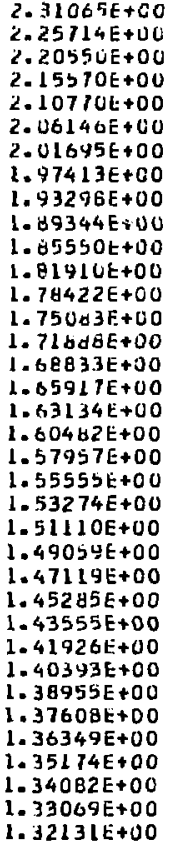 & $\begin{array}{l}2.535 \\
2.412 \\
2.411 \\
2.353 \\
2.290 \\
2.242 \\
2.149 \\
2.115 \\
2.090 \\
2.041 \\
1.994 \\
1.455 \\
1.914 \\
1.874 \\
1.836 \\
1.800 \\
1.765 \\
1.731 \\
1.700 \\
1.664 \\
1.640 \\
1.6113 \\
1.581 \\
1.54\end{array}$ \\
\hline
\end{tabular}

2.1 COUUE $+00 \quad 2.20000 E+00$ 
GAMHA $=1+(1-W)((2 S R /(1-h))(A H C G / H M C)-1)((1-W) R H C / R H C G)+\$ 5+W(2 S W-2)$

$$
5 R=1.30 \quad \mathrm{RHCG}=2.40 \quad \mathrm{SK}=1.20
$$

RHO $\$ 1.00000 t+00 \quad 1.10000 E+00 \quad 1.20000 E+C O \quad 1.30000 t+00 \quad 1.40000 E+00 \quad 1.50000 E+00 \quad 1.80000 E+00 \quad 1.70000 E+00$ W

\begin{tabular}{|c|c|c|c|c|c|c|c|c|}
\hline $\begin{array}{l}00000 E-02 \\
00000 E-02 \\
00000 t-02 \\
00000 E-02 \\
00000 E-02 \\
00000 E-02 \\
00000 E-01 \\
10000 E-01 \\
20000 E-01 \\
30000 E-01 \\
40000 t-01 \\
50000 F-01 \\
60000 t-01 \\
70000 t-01 \\
80000 t-01 \\
90000 E-01 \\
00000 t-01 \\
10000 E-01 \\
20000[-01 \\
30000 t-01 \\
40000 t-01 \\
50000 t-01 \\
60000 t-01 \\
70000 t-01 \\
80000 t-C 1 \\
90000 t-01 \\
00000 t-01 \\
10000 t-01 \\
20000 t-01 \\
30000 E-01 \\
40000 E-01 \\
500001-01 \\
60000 t-01\end{array}$ & 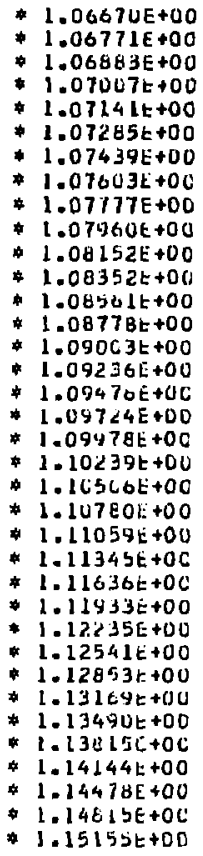 & 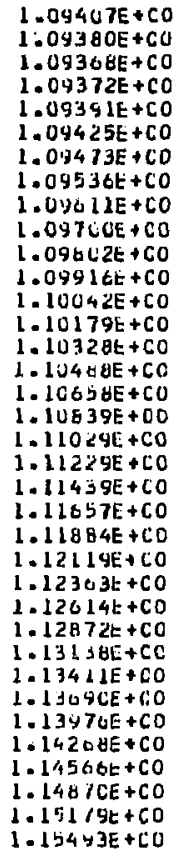 & 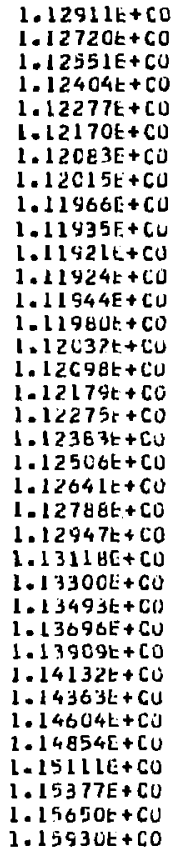 & $\begin{array}{l}1.172 \times 5 E+00 \\
1.144 U 1 E+00 \\
1.16536 E+00 \\
1.1620 U E+00 \\
1.15891 E+00 \\
1.15610 E+00 \\
1.15354 E+00 \\
1.15124 E+00 \\
1.14919 E+00 \\
1.14718 E+00 \\
1.14581 E+00 \\
1.14446 E+00 \\
1.14134 E+00 \\
1.1423 E+00 \\
1.1417 E+00 \\
1.14122 E+00 \\
1.14042 E+00 \\
1.14040 E+00 \\
1.14087 E+00 \\
1.14112 E+00 \\
1.14154 E+00 \\
1.14212 E+00 \\
1.14287 t+00 \\
1.14377 E+00 \\
1.14481 E+00 \\
1.14601 E+00 \\
1.14734 E+00 \\
1.1481 E+00 \\
1.15041 E+00 \\
1.15213 E+00 \\
1.15397 t+00 \\
1.15593 E+00 \\
1.15800 E+00 \\
1.16018 E+00 \\
1.16245 E+00 \\
1.16443 E+00\end{array}$ & 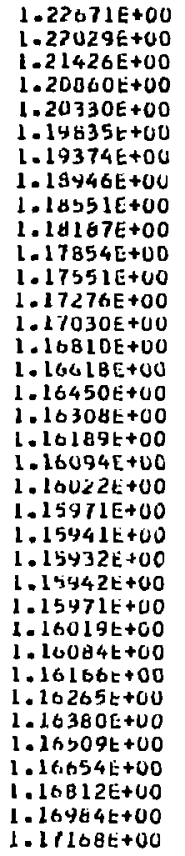 & 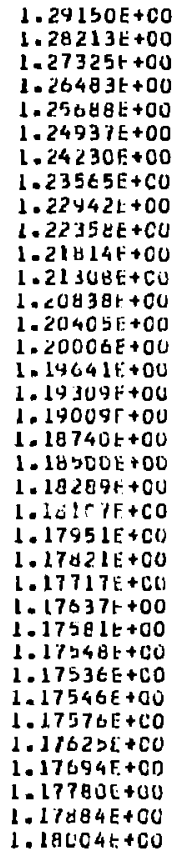 & 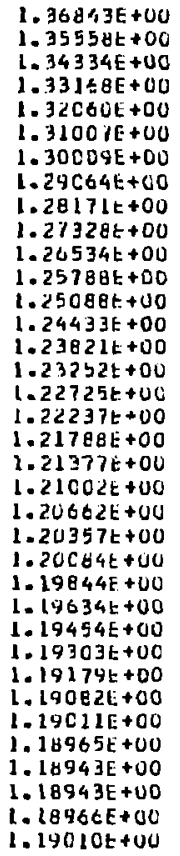 & $\begin{array}{l}1.45856 E+00 \\
1.44168 E+00 \\
1.42553 E+00 \\
1.41010 E+00 \\
1.39538 E+00 \\
1.38134 E+00 \\
1.36797 E+00 \\
1.35526 E+00 \\
1.34318 E+00 \\
1.33172 E+00 \\
1.32087 E+00 \\
1.31020 E+00 \\
1.30091 E+00 \\
1.29176 E+00 \\
1.28316 E+00 \\
1.27509 E+00 \\
1.26752 E+00 \\
1.26045 E+00 \\
1.25386 E+00 \\
1.24773 E+00 \\
1.24205 E+00 \\
1.23682 E+00 \\
1.23200 E+00 \\
1.22759 E+00 \\
1.22358 E+00 \\
1.21996 E+00 \\
1.21670 E+00 \\
1.21380 E+00 \\
1.21175 E+00 \\
1.20403 E+00 \\
1.20713 E+00 \\
1.20553 E+00 \\
1.20424 E+00 \\
1.20323 E+00 \\
1.20250 E+00 \\
1.20203 E+00\end{array}$ \\
\hline
\end{tabular}


GAMHA $=1+(1-W)(125 R /(1-h))(H H E G / R H[)-1)((1-W) K H C / H H C G) * 5+W(2 S H-2)$

$$
S_{R}=1.30 \quad \text { RHCE }=2.40 \quad S w=1.20
$$

RHC $1.80000 E \div 00 \quad 1.90000 E+C O \quad 2.00 C O O E+C O \quad 2.10000 E+00 \quad 2.20000 E+00$

$2.40000 t+00$ H

\begin{tabular}{|c|c|c|c|c|c|c|c|}
\hline $\begin{array}{l}1.00000 \mathrm{E}-02 \\
2.00000 \mathrm{E}-02 \\
3.00000 \mathrm{E}-02 \\
4.00000 \mathrm{E}-02 \\
5.00000 \mathrm{E}-02 \\
6.00000 \mathrm{E}-02 \\
7.00000 \mathrm{E}-02 \\
8.00000 \mathrm{E}-02 \\
9.00000 \mathrm{E}-02 \\
1.00000 \mathrm{E}-01 \\
1.10000 \mathrm{E}-01 \\
1.20000 \mathrm{E}-01 \\
1.30000 \mathrm{E}-01 \\
1.40000 \mathrm{E}-01 \\
1.50000 \mathrm{E}-01 \\
1.60000 \mathrm{E}-01 \\
1.70000 \mathrm{E}-01 \\
1.80000 \mathrm{E}-01 \\
1.90000 \mathrm{E}-01 \\
2.00000 \mathrm{E}-01 \\
2.10000 \mathrm{E}-01 \\
2.20000 \mathrm{E}-01 \\
2.30000 \mathrm{~F}-01 \\
2.40000 \mathrm{E}-01 \\
2.50000 \mathrm{t}-01 \\
2.60000 \mathrm{t}-01 \\
2.70000 \mathrm{E}-01 \\
2.80000 \mathrm{E}-01 \\
2.90000 \mathrm{E}-01 \\
3.00000 \mathrm{E}-01 \\
3.10000 \mathrm{E}-01 \\
3.20000 \mathrm{E}-01 \\
3.30000 \mathrm{t}-01 \\
3.40000 \mathrm{E}-01 \\
3.50000 \mathrm{E}-\mathrm{C} 1 \\
3.60000 \mathrm{E}-01\end{array}$ & 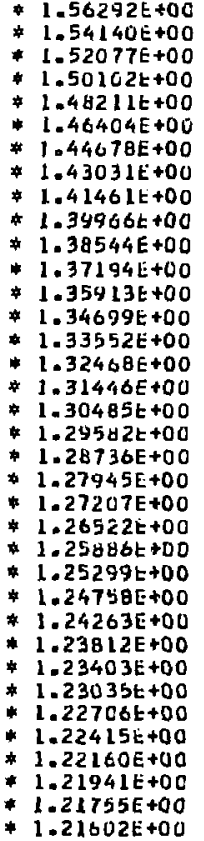 & 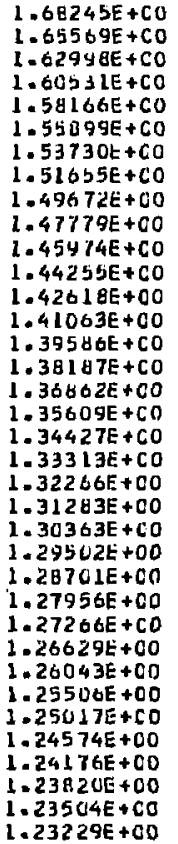 & 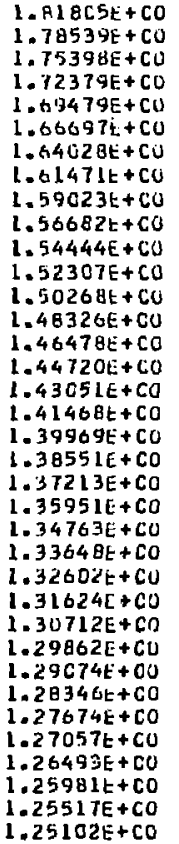 & $\begin{array}{l}1.97048 E+00 \\
1.93128 E+00 \\
1.89353 E+U U \\
1.8572 U E+00 \\
1.82226 E+00 \\
1.78808 E+00 \\
1.75643 E+00 \\
1.72548 E+00 \\
1.64580 E+00 \\
1.66737 E+00 \\
1.64014 E+00 \\
1.61410 E+00 \\
1.58922 E+00 \\
1.56546 E+00 \\
1.54280 E+00 \\
1.52120 E+00 \\
1.50065 E+00 \\
1.48111 E+00 \\
1.46255 E+00 \\
1.44495 E+00 \\
1.42628 E+00 \\
1.41252 E+00 \\
1.39763 E+00 \\
1.38359 E+00 \\
1.37038 E+00 \\
1.35797 E+00 \\
1.34633 E+00 \\
1.33544 E+00 \\
1.32527 E+00 \\
1.31581 E+00 \\
1.30702 E+00 \\
1.29886 E+00 \\
1.29137 E+00 \\
1.28447 E+00 \\
1.27815 E+00 \\
1.27240 E+00\end{array}$ & $\begin{array}{l}2.14045 E+00 \\
2.04405 E+00 \\
2.04932 E+00 \\
2.00622 E+00 \\
1.96471 E+00 \\
1.9247 E+00 \\
1 . B 8638 E+00 \\
1.84947 E+00 \\
1.81404 E+00 \\
1.78004 E+00 \\
1.74145 E+00 \\
1.71622 E+00 \\
1.66033 E+00 \\
1.65775 E+00 \\
1.63044 E+00 \\
1.60437 t+00 \\
1.51951 E+00 \\
1.55543 E+00 \\
1.53330 E+00 \\
1.51188 E+00 \\
1.49154 E+00 \\
1.47226 E+00 \\
1.45401 E+00 \\
1.43674 E+00 \\
1.42045 E+00 \\
1.40508 E+00 \\
1.39662 E+00 \\
1.37704 E+00 \\
1.36431 E+00 \\
1.35239 E+00 \\
1.34127 E+00 \\
1.33092 E+00 \\
1.32131 E+00 \\
1.31240 E+00 \\
1.30419 E+00 \\
1.29664 E+00\end{array}$ & $\begin{array}{l}2.32851 E+C O \\
2.27426 F+00 \\
1.22190 E+00 \\
2.1714 C E+C 0 \\
2.12272 E+00 \\
1.07582 E+00 \\
1.03067 E+C 0 \\
1.98724 E+00 \\
1.94549 E+00 \\
1.90537 E+00 \\
1.66087 E+00 \\
1.82993 E+00 \\
1.71453 E+00 \\
1.76063 E+00 \\
1.72619 E+00 \\
1.61718 E+00 \\
1.66758 E+00 \\
1.43930 E+00 \\
1.61236 E+00 \\
1.58671 E+00 \\
1.56231 E+00 \\
1.53913 E+00 \\
1.31713 E+00 \\
1.49628 E+C U \\
1.47655 E+00 \\
1.45790 E+00 \\
1.44030 E+00 \\
1.42372 F+00 \\
1.40812 E+00 \\
1.3934 B E+00 \\
1.37476 E+00 \\
1.36693 E+00 \\
1.35496 E+00 \\
1.34383 E+C 0 \\
1.33349 E+00 \\
1.32392 E+00\end{array}$ & $\begin{array}{l}2.53509 E+00 \\
2.47235 E+00 \\
2.41174 E+00 \\
2.35321 E+00 \\
2.29674 E+00 \\
2.24228 E+00 \\
2.1898 C E+00 \\
1.13926 E+00 \\
2.09061 E+00 \\
2.04383 E+00 \\
1.99867 E+00 \\
1.95570 E+00 \\
1.91427 E+00 \\
1.67454 E+00 \\
1.83648 E+00 \\
1.80005 E+00 \\
1.76521 E+00 \\
1.73192 E+00 \\
1.70013 E+00 \\
1.66982 E+00 \\
1.64095 E+00 \\
1.61347 E+00 \\
1.58734 E+00 \\
1.56254 E+00 \\
1.53401 E+00 \\
1.51674 E+00 \\
1.49566 E+00 \\
1.47576 E+00 \\
1.45700 E+00 \\
1.43933 E+00 \\
1.42273 E+00 \\
1.40716 E+00 \\
1.34257 E+00 \\
1.31895 E+00 \\
1.36626 E+00 \\
1.35445 E+00\end{array}$ \\
\hline
\end{tabular}


GAMMA $=1+(1-W)(125 H /(1-h))($ RHCG/RHC)-1)(1)-h)RHC/RFCG)*\$5 +W(25h-2)

$S R=1.3 U \quad K H[G=2.43 \quad S W=1.20$ $+$

$1.00000 \mathrm{E}-02 * 1.063 \mathrm{EOE}+00$ $2.00000 \mathrm{E}-02 * 1.06455 \mathrm{E}+00$ $3.00000 t-02 * 1.06620 t+00$ $4-00000 t-02 * 1.06756 t+00$ $5.00000 \mathrm{E}-02+1.06902 \mathrm{t}+00$ $6.00000 t-02+1.07054 t+00$ $7.00000 t-02 * 1.07224 t+00$ $8.00000 t-02+1.07399 t+00$ $1.00000 t-02 * 1.07593 E+00$ $1.00000 E-01 * 1.07776 t+00$ $1.10000 t-01+1.07977 E+00$ $1.20000 E-01$ - $1.08167 E+00$ $1.30000 t-01 * 1.08405 E+00$ $1 .+0000 t-01 * 1.08630 t+00$ $1.30000 r-01 \cdot 1.08063 E \$ 00$ $1.60000 t-01 * 1.09104 t+00$ $1.70000 t-01 * 1.09351 t+00$ 1. BuOUUt-01*1.09806t+00 $1.90000 L-01 \cdot 1.04867 E+00$ $2.00000 E-01+1.10174 t+00$ $2.10000 E-C 1 * 1.1040$ Bt+OC $2.20000[-01$ 1. $10467 t+0 C$ $2.30000 t-01 * 1.10472 t+00$ $2-400005-01+1.11263 t+00$ 50000t-01 1.11559t+00

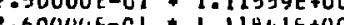

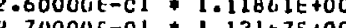
. . $2.90000 t-01$. $1.12794 t+00$ $3.00000 t-01.1 .13114 t+00$ .10000t-or 1.1 $3.20000 l-a 1 * 1.13767 t+00$ $3.30000 \mathrm{t}-0 \mathrm{l}$ 1.14100t+00 $3.40000 \%-01+1.1443 a k+00$ $3.50000 \mathrm{r}-0 \mathrm{l} * 1.14716 \mathrm{t}+0 \mathrm{C}$
$3.60000 \mathrm{t}-01+1.15120 \mathrm{t}+0 \mathrm{C}$

1.10040

$1.0894 \mathrm{JE}+\mathrm{CO}$ $1.0 H 9 \cup 5 E+C O$ $1.0894 \angle E+C D$ $1.04014 E+C O$ 1. $0905 \mathrm{CE}+\mathrm{CO}$ 1. UY I CLE + CO $1.09165 E+C O$ $1.09243 E+C O$ $1.04333 E+C O$ $1.044 J 6 E+C$ $1.04532 E+C 0$ $1.09679 E+C O$ $1.04+17 E+C$ $1.094676+C 0$ 1. $1 \cup 1 \angle 7 E+C O$ 1. $114798+C D$ I. IĆÓ $7 O E+C$ 1. $10070 E+C$ $1.11079 E+C$ $1.11247 t+C 0$ 1. $115<4 E+C O$ $1.11734 t+C 0$ 1. $1246 \sin ^{2} t+c 0$ $1.12252 t+C 0$ $1.1251(4)+C O$ $1.1251 \mathrm{CE}+\mathrm{CO}$ 1. $1304 \mathrm{dE}+\mathrm{CO}$ $1.13326 E+C$ $1.13611 E+C O$ 1. $11902 E+C O$ $1.14199 \mathrm{E}+\mathrm{CO}$ $1.14542 E+C O$ 1. $14810 E+C O$ $1.151<3 E+C O$
$1.15442 t+C O$

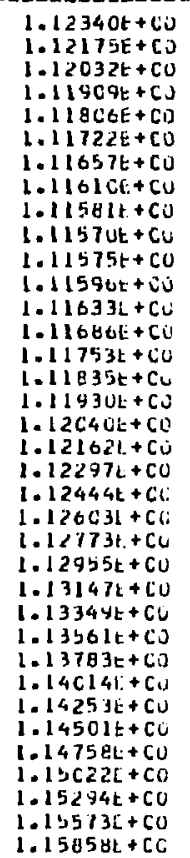

1. $16528 \mathrm{E}+0 \mathrm{U}$

$1.40000 E+00$ 1.300005:001 1. COCOCE +OU 1. ICOCOE+OO I. I6IOYE+00 I.21U7IE+U0 1. Isasot+00 1.20512t+00 -1SS3SE+UO $1.19988 t+00$ 1.15258E+CO $1.19499 E+C 0$ $1.14781 E+00$ 1.1862?t+00 $1.14579 E+001.182315+00$ 1. $14402 E+00$ l. $17871 \mathrm{~L}+00$ $1.14247 E+001.178715+00$ 1.14247Et00 $1.17 b 425+00$ $1.14119 E+00$ 1.1724lE+OO 1.14004k+00 $1.16369 t+00$ $1.13915 t+00$ 1.16725t+U0 $1.13846 E+0 C \quad 1.16308 t+00$ 1. $13777 E+00$ 1.1631BE+UO $1.13767 E+00$ 1.16150E+130 $1.13750 t+\mathrm{LO} \quad 1.100 \mathrm{C} \theta t+\mathrm{LO}$ $1.13704 E+00$ 1.15AYOt+UO $1.13700 t+00$ 1.15745t+00 $1.13 A .30 E+00$ L.I5723E+UO

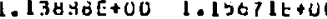
$1.13+n 2 E+00 \quad 1.13641 L+00$ 1. $14031 t+00$ 1.15b $31 E+00$ $1.141360+00 \quad 1.15640 E+U 0$ $1.14274 \mathrm{E}+0 \mathrm{U}$ 1.1504HE+OD 1.14274E+00 1. $15008 E+00$ $1.14406 \mathrm{t} 00$ 1.15T14t+0 $1.14352 E+00$ 1.1 1.14 1. $14881 E+00$ 1. $15955 \mathrm{E}+00$ 1.15ub4e+UO 1.1hubae+00 1.1525detuO I.16IS5E+UO 1. $15403 t+0 D \quad 1.16337 t+00$ $1.156 / 9 t+00 \quad 1.16493 L+00$ $1.15905 t+00$ 1.1ABO $3[+00$ $1.16141 t+00 \quad 1.16845 t+00$ $1.27862 C+00$ $1.26983 \mathrm{E}+\mathrm{CO}$ 1.35222E+UU $1.26151 E+C O$ 1. $14 C 1 C t+00$ $1.25365 F+00 \quad 1.31753 t+00$ $1.24622 t+0 U$ I. $3071 t+t U C$ $1.23922 \mathrm{t}+\mathrm{L} C \mathrm{C} \quad 1.29727 \mathrm{t}+0 \mathrm{O}$ $1.23263 t+00 \quad 1.28740 t+00$ $1.220465+001.27904 t+00$ $1.2064 t+C 41.27104+00$ $1.71528 t+C O \quad 1.20277 L+U 0$ $1.21025 t+00 \quad 1.25594 t+00$ 1. $24500 \mathrm{~L}+00 \quad 1.2484 \angle \mathrm{L}+00$ $1.201241+C 0 \quad 1.24151 t+00$ $1.1 .733 t+C 0$ 1. $19373 t+c 0$ 1. $14039 L+L U$ $1.184711+00$ 1. $\mathrm{L}=23 \mathrm{Litcu}$ $1.130206+00$ $1.17037 \mathrm{~F}+\mathrm{LO}$ 1. I7nE11.+UO $1.11550 t+C U$ 1. $17444 t+C u$ 1. $13435+c 0$ $1,1730 \mathrm{bt}+\mathrm{Cu}$ $1 \cdot 11269 t+C 0$ - 17250t+00 $1.112631+00$ $1.17200+0 \mathrm{CO}$ 1. $17290+. c 0$ $1.174 C 2 t+C u$ 1. $17486 \mathrm{E}+\mathrm{C}$ $1.17527 \mathrm{t}+\mathrm{C}$ 1. $33582 t+U U$ $1.2901 E E+O U$ $1.2 \times 430 t+00$ $1.22 C \mathrm{C} 3 t+u G$ 1. $21555 t+u t$ $1.21144 r+00$ 1.2u7tst+Uu 1. $2(1424 t+0 L$ $1.21122 c+00$ 1. $1984 E L+C O$ $1.19646 t+00$ $1.14354 E+00$ $1.19394 E+00$ 1 140581000 I $4032 t+0$ - 104 tit +00 104 $1.18757 c+00$ 1. 1. IUtezt +uU 1. Iot 7 tt +UO $1.1669 / 1+00$ $1.187371+00$ $1.178 \geq 71+60 \quad 1.147575+06$

$1.4385 U E+00$ $1.42251 t+00$ $1.40723 E+00$ 1. $37872 \mathrm{E}+\mathrm{UC}$ 1. $.157 \mathrm{HBE}+010$ 1. $3408 \mathrm{CL}+0 \mathrm{O}$ 1. $12448 E+00$ 1. 11 A OEE + DU 1. $3 C 649 E+U 0$ 1. $24894 t+U 0$ 1. $24975 \mathrm{E}+00$ $1.28118 \mathrm{~B}+10$ $1.27314 E+U U$ I. 2 ESSTE+00 !. $75853 E+00$ -?. $195 E+U$ . $24563 E+00$ $1.24015 E+U 0$ $1.23490 E+60$ $1.23007 E+00$ 1.225esE+00 $1.221+2 t+40$ 1.2179PE+00 $1.21408 t+v 0$ 1. $1175 E+00$ 1. 2 C I. $2040 \mathrm{CE}+00$ 1. 20440 t 1. $.033 \angle E+00$ 1. $2 \mathrm{Cl} 4 \mathrm{HE}+\mathrm{BO}$ 1. $2 \cos 3 t+00$ $1.2 C 014 E+00$ 1. $15430 E+U 0$ 


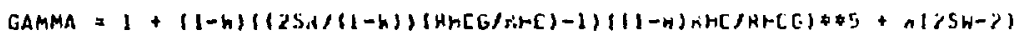

$$
S H=1.3 \mathrm{C} \quad \mathrm{BHCO}=2.43 \quad \mathrm{SH}=1.2 \mathrm{C}
$$

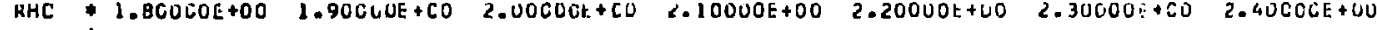

$1.00000 \mathrm{t}-02 * 1.53345 \mathrm{E}+00$ $2.00000 \mathrm{E}-02+1.51801 \mathrm{E}+00$ (1.0000 $5.00000 \mathrm{E}-02$ 1. $1.46176 \mathrm{~B}+00$ 5.00000E-02 $1.46176 E+00$ $6.000001-02+1.44463 k+00$ 7.00000 $8.00000 E-02+1.41269 E+00$ $9.00000[-02 * 1.397 \Delta 4 E+00$ $1.00000 E-01 * 1.3837 U E+00$ $1.10000 E-01 * 1.3702 Z E+00$
$1.20000 E-01 * 1.35753 E+00$ $1.30000 \mathrm{E}=01 * 1.34545 E+00$ $1.40000 E-01 * 1.33402 t+0$ 1.50000 E-01 $* 1.32321 E+00$ $1.60000 F-01 * 1.31302 E+00$ $1.70000 \mathrm{t}-0 \mathrm{~L} * 1.30343 \mathrm{E}+00$ $1.80000 \mathrm{E}-01+1.2944 \mathrm{LE}+00$ $1.90000 E-01 * 1.28595 E+00$ $2.00000 E-01 \quad 1.278045+00$ 2.10000 01 1.2705E 1.27065E+00 $2.20000 E-01$ 1.2637BE +00 $2.30000 \mathrm{E}-01 * 1.25740 \mathrm{t}+00$ $2.40000 t-01 * 1.25150 \mathrm{t}+00$ 2.50000 ed *1.24607e+00 $2.60000 \mathrm{E}-01$ 1.24108E+00 $2.70000 t-01 * 1.23653 t+00$ $2.80000 t-01 * 2.23239 t+00$ $2.90000 F-01 * 1.22866 E+00$ $3.00000 E-01 * 1.225+7 E+00$ .1000OE-02 *1.2223bE+OD $3.20000 E-01 * 1.21476 E+00$ $3.30000 E-01$ 1.21751E+00 $3.40000 \mathrm{E}-01 \div 1.21560 \mathrm{E}+00$ $3.50000 E-01 * 1.21401 E+00$ 3.60000E-01
$1.653 U 1 E+C O$ $1.62732 \mathrm{E}+\mathrm{CO}$ $1.603 \cup 6 E+C O$ $1.57900 E+C O$ $1.55111 E+C O$ $1.5355 \mathrm{EE}+\mathrm{CO}$ 1. 4 15 $27 E+C$ $1.47640 E+C D$ $1.45 B 51 E+C$ 1. $44140 \mathrm{E}+\mathrm{C}$ $1.42512 E+C$ $1.40963 E+C O$ $1.39442 E+C O$ 1. 3 $1046 E+C$ $1.36774 E+C$ l. $35524 \mathrm{E}+\mathrm{CO}$ $1.3434 \mathrm{JE}+00$ 1. $33230 E^{\circ}+C O$ 1. $32182 E+C O$ 2. $311 \mathrm{BE}+\mathrm{CO}$ 1.31198E+CO 1.302 r $5 E+C O$ 2. $29413 E+C$ $1.28600 E+C$ 1.27639E+CO $1.27165 E+C O$ 1. $20523 E+C O$ $1.25932 E+C D$ $1.25369 t+00$ $1.24894 E+00$ I. $24445 E+C D$ I. $24040 E+C O$ $1.23677 E+C O$ $1.23354 E+00$ $1.23071 E+C O$ 1 . 22826 GE + CO
$1.783 C 3 E+C O$ $1.75189 t+C u$ $1.72194 t+C 0$ $1.69317 t+C D$ $1.69317 \mathrm{E}+\mathrm{CO}$ $1.66555 E+C O$ $1.63905+C O$ $1.61366 E+C O$ $1.58933 \mathrm{t}+\mathrm{CU}$ $1.56605 E+C U$ $1.54379 i+C U$ $1.52252 E+C O$ $1.50223 \mathrm{E}+\mathrm{CO}$ $1.48288 E+C O$ $1.46446 E+C U$ $1.44693 E+C O$ $1.4302 \mathrm{dt}+\mathrm{CO}$ 1. $4144 \mathrm{BE}+\mathrm{CO}$ $1.34951 \mathrm{~L}+\mathrm{CO}$ $1.38533 E+C O$ $1.37194 t+C$ $-35931 t+C$ $1.34741 t+C$ $1.33622 t+00$ $1.32573 \mathrm{E}+\mathrm{C}$ . $31590 \mathrm{t}+\mathrm{C}$ - $30673 E+C D$ 1.? $98 \mathrm{~L}$ aE + 0 $1.29023 E+C$ $1.28289 t+C a$ l. $27604 E+C$ $1.26984 \mathrm{E}+\mathrm{C}$ $1.26413 E+C$ $1.25892 \mathrm{E}+\mathrm{CO}$ $1.25420 \mathrm{E}+\mathrm{CO}$ $1.24995 t+C 0$ $1.24615 E+C$
$1.92431 E+C C \quad 2.092 b 2 t+00$ 1. $89286 E+00$ 1. $85582 E+00$ 1. $78780 \mathrm{E}+00$ $1.78780 E+00$
$1.75577 E+00$ 1. $72502 \mathrm{E}+00$ $1.9552 E+00$ $1.66724 E+00$ $1.64016 \mathrm{E}+00$ $1.61424 \mathrm{E}+00$ 1. $58946 \mathrm{t}+00$ 1. $56519 \mathrm{E}+00$ $1.54320 t+00$ 1. $52146 E+00$ $1.50125 E+00$ $1.48164 E+00$ $1.46311 \mathrm{E}+00$ $1.4455 L E+00$ $1.42884 E+00$ $1.42884 E+00$ $1.41306 E+00$ $1.39814 E+00$ 1. $38407 E+00$ 1. 37001 to I. $35835 \mathrm{E}+\mathrm{UO}$ 1. $34665 \mathrm{E}+00$ $1 \cdot 33569 E+00$ 1. $32545 E+00$ 1. $31540 E+00$ 1. $30702 E+00$ 1. $29880 E+00$ $1.29119 \mathrm{E}+00$ 1. $28419 E+00$ 1. $27777 E+00$ $1.27191 E+00$ 1. $26659 E+00$ $2.04814 t+U 0$ $2.00537 E+00$ 1. $96417 t+00$ $1.92451 E+00$ $1.94068 \mathrm{t}+0$ 1. $1446 t+00$ 1. $74 \mathrm{~d} 21 \mathrm{E}+00$ $1.71713 \mathrm{t}+00$ -580a5t+00 $.55720 t+00$ $.53469 t+00$ $1.51328 E+00$ $1.49294 \mathrm{E}+00$ $1.45535 E+00$ $43804 E+00$ $1.42169 E+00$ $1.40626 \mathrm{t}+00$ $1.36525 \mathrm{E}+00$ $1.35324 E+00$ 1. $34202 E+00$ $1.33156 E+00$ $1.321 B 3 E+00$ - $31282 E+00$ $1.30448 t+00$ $1.29680 \mathrm{t}+00$ $1.28976 E+00$
$2.273241+00 \quad 2.41197 E+00$ $\angle .22124 t+C C \quad 2.41177 t+00$ $2.17116 t+C U \quad 2.33367 C+00$ $2.12282 E+C O \quad 2.29758 E+00$ $2.07624 E+C O \quad ? .24348 t+00$ $2.03136 \mathrm{t}+\mathrm{CO} 2.14133 t+0$ $1.946545+C 0$ 2. $042 \%$ $1.946679+400$ $1.506791 .+002.046162+00$ $1.56047 .00 \quad 2.00141 E+00$ $1.83170 t+00 \quad 1.95842 t+00$ $1.91715 E+00$ 1.7fi265t+CO $1.87756 t+00$ 1.730j1t+CO $1.85962 t+U 0$ $1.62938 E+C U$ 1.80327t +UU $1.66482 t+C 0 \quad 1.76850 t+00$ $1.6416 U E+C O \quad 1.73525 E+00$ $1.61469 t+00 \quad 1.70350 t+00$ 1. $589 C 4 E+C O \quad 1.6732 C E+00$ $1.56+63 E+C 0 \quad 1.64431 E+00$ $1.541430+C 0 \quad 1.616805+00$ $1.514405+00$ 1.54064E+CO $1.49850 t+00$ 1.5057 $1.470710+00$ 1. $54214 E+00$

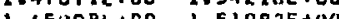
$1.45998 \mathrm{~d}$ CD $1.51982 E+00$ $1.44230 t+00$ 1.49666t+00 $1.42563 E+00$ I.47E65E+00 $1.40493 E+00$ I.45477E+0O $1.34518 E+C O \quad 1.44197 E+00$ $1.38134 E+00 \quad 1.42524 E+00$ $1.36839 t+00$ 1.40952E+00 $1.35629 E+00$ 1. $3947 \mathrm{BE}+00$ $1.34501 E+00$ I. $38101 E+00$ . $33454 \mathrm{E}+00$ L.36819E +00 1. 35617t+CO 
GAMHA $=1+(1-h)(12 S A /(1-h))(R H O G / R H C)-1)(1)-h) K H C / R H C G)+\$ 5+W(25 h-2)$

$S R=1.3 \mathrm{C} \quad \mathrm{RHCC}=2.50 \quad S W=2.20$

W

RHC

1

$1.00000 \mathrm{E}-0 \mathrm{O}$

$2.00000 \mathrm{t}-02 * 1.05766 \mathrm{E}+00$ 政 .00000E-02 $1.0622 \mathrm{GE}+0 \mathrm{C}$ $6.00000 t=02 * 1.06398 E+00$ .0000 $1.0657 \mathrm{st}+00$ $02 \div 1.067 t B E+00$ $8.00000 E-02+1.06966 E+00$ $.00000 E-02 \$ 1.07172 E+00$ $1.00000 \mathrm{E}-01.1 .07386 \mathrm{a}+00$ $1.10000 t-01$ 1.07ouge+00 $1.2000 u t-01$ * $1.078 .37 t+00$ $1.30000 t-01+1.08073 t+00$ $1.40000=01+1.00317 E+00$ $1.50000[-0]+1.08567 t+00$ $1.60000 E-01 * 1.08824 E+00$ $1.70000 E \sim 01+1.09067 t+00$ $1.80000 t=01+1.09356 t+00$ $1.90000 t-01+1.09632 t+00$ $2.00000 t-01+1.09413 E+00$ $2.10000 \mathrm{E}-01+1.10199 \mathrm{E}+00$ $2.20000 \mathrm{k}-01+1.10491 \mathrm{k}+00$ $2.30000 \mathrm{r}-01$ 1.1078 $3.40000 \mathrm{E}-01$ i. $2.50000 E-01$ 1.113.7t+00

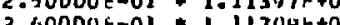
2.70000L-01 1.12025t+00 2. 2.00000 1. ilavortao 3.00000 - 01 1.129yetod 3. $3.20000 E-01$ *1.13606t+00 $3.30000 t-01 * 1.14006 \mathrm{t}+00$ $3.40000 E-01+1.14349 c+00$ $3.50000 t-01 * 1.14655 t+00$

1. $10000 E+C$

1. $20 \mathrm{CODE}+$

$1.30000 E+00$

1. $40000 E+00 \quad 1.50000 E+00$

$1.60000 t+00$

1. $70000 E+00$

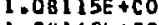
$1.0,148 t+C O$ $1.08195 E+C O$ 1. OUZ2 S5E + CO $1.08328 E+C D$ 1.084 1 4t + $+C 0$ $1.09513 f+C 0$ $1.08623 t+C O$ $1.08745 E+C D$ $1.08878 E+C D$ $9022 E+C D$ 1. $04177 E+C O$ $1.0 \rightarrow 342 E+C D$ $1.0+517 t+C 0$ $1.09702 E+C O$ L. 094 YQEE +CO 1. $100 \times 95+C 0$ $1.10312 t+\mathrm{CO}$ $1.10532 t+C 0$ $.104 \% 6 E+C O$ $1.11242 E+C O$ $1.114445+C 0$ $1.11494 E+C 0$ $1.11733 E+C 0$ $1.12019 t+C O$ $1.12571 E+C C$ $1.128 b G t+C O$ 1.1314 IE +CO $1.13444 E+C D$ $1.13746 E+C O$ 1. $14044 E+C O$ 1. $143 C 7 E+C O$ 1. $14604 E+C O$ 1. $12 C C G E+C O$ l. $15333 \mathrm{E}+\mathrm{CO}$

\section{$1.11127 E+C 0$} 1. $11 \mathrm{Cl} 9 \mathrm{t}+\mathrm{C}$ $1.10930 E+C$ l. $10807 t+C u$ 1.10771E + C $1.10753 \dot{x}+C$ $1.10752 t+c$ $1.10766 \mathrm{E}+\mathrm{CO}$ 1. $16796 E+C O$ 1. $10441 E+C O$ $1.10500 E+C O$ $1.10574 E+C O$ $1.11163 L+C U$ $1.11277 t+C$ $1.114 C 4 E+C N$ L. $11542 t+C U$ I. $11693 t+C j$ 1. $1185 b E+C O$ $1.12 C 28 E+C D$ $1.12211 t+C$ $1.12405 t+C U$ $1.12405 \mathrm{t}+\mathrm{CD}$
$1.126 \mathrm{COE}+\mathrm{CO}$ $1.12622 E+C 1$ $1.12622 E+C$ 1.13c44L+co $1.13516 t+C O$ 1. 37 AE +CO $1.142841+C 0$ $1.14284 t+C O$ $1.14555 t+C L$ $1.14831 t+C 0$ $1.15118 \mathrm{E}+\mathrm{CO}$ $1.154 C 4 i+C 0$ $1.157 \mathrm{C} 7 \mathrm{E}+\mathrm{CO}$

\section{$1.14899 E+00$} 1. $14616 E+00$ $1.14358 E+0 U$ $1.14124 E+00$ $1.13415 E+00$ $1.13729 t+00$ i. $13565 t+00$ 1. $13565 \mathrm{E}+00$ $1.13304 E+00$ 1.13 1.13205e+00 $1.13126 \mathrm{ta0}$ 1. $130 u 7 E+00$ $1.13026 t+00$ $1.13005 t+00$ $1.13001 E+00$ I. $13015 E+00$ $1.13045 t+00$ 1. $130 \times 2 \mathrm{E}+00$ 1. $13155 E+00$ $1.23233 E+00$ I. $13325 E+0$ $1.13432 \mathrm{E}+\mathrm{O}$ $1.13553 \mathrm{E}+0$ 1. $135 \mathrm{FE}+0$ 1. $136 a 7 E+0$ $1.13832 E+0$ i. $14100 E+00$ $1.1434 \mathrm{YE}+0$ - $14943 \mathrm{E}+00$ 1. $14903 E+0$ - 151005 1. $15423 E+00$ 1. $15666 \mathrm{E}+00$ 1. $15919 E+O D$ 1. 161 BLE + UO

\section{$1.19531 E+00$} $1.19034 E+00$ -18570E+00 $1.177376+00$ $1.173665+00$ 1.17) $1.17425 E+00$ $1.16713 E+00$ $1.16429 \mathrm{E}+00$ $1.16172 E+00$ 1.15441Et+00 $1.15736 t+00$ $1.15556 E+00$ 1.15401t+00 1. $15268 E+00$ $1.15159 \mathrm{~L}+00$ $1.15071 E+00$ 1. $15005 E+00$ $1.14960 E+00$ $1.24935 t+00$ $1.14429 E+00$ $1.14942 t+00$ $14973 E+00$ .14973E+00 - $15022 E+U$ $1.1504 B E+O 0$
$1.15170 E+00$ I. $15267 E+00$ $1.15380 c+00$ - IJ SU8E+OO - 15650E+00 1. $19009 t+00$ $1.15973 E+00$ $1.16154 \mathrm{E}+0$ $1.16 .347 \mathrm{t}+00$ 1. $10251 E+00$ $1.16 .767 i+00$
$1.25124 E+00$ $1.24370 t+00$ $1.23659 E+00$ $1.22488 \mathrm{t}+\mathrm{CO}$ $1.22357 E+C 0$ $1-21765 \mathrm{E}+00$ $1.21211+c 0$ 1.20093E+c0 1. 1.iv7se 1. $18971 \mathrm{t}+00$ 1. $14623 \mathrm{E}+00$ 1. $18306 \mathrm{E}+00$ 1. $18 O 28 E+C O$ $1.17760 E+00$ $1.17531 t+00$ 1.17328E+OO 1. $17153 E+00$ $1.17003 t+00$ $1.1587 a++60$ $1.16777 t+C 0$ $107005+00$ 1.16700t00 1.10612E:CD 1. 16600400 1. l. 1163971000 1. 167485000 . $16831 E+00$ $1.16 y 30<+00$ $1.17046 t+00$ $1.17177 E+C O$ $1.17323 F+00$ $1.17484 E+C 0$
$74 t+00$ . 3071 EE +00 1. 3e171E+00 $.29715 E+00$ 1. $36828 E+00$ $1.28762 E+001.35547 E+00$ $.27800 E+00$ 1.34328E+00 $1.270005+00 \quad 1.331696+00$ $1.261906+40$ 1. $320005+00$ $1.25438 E+00$ l. $310236+00$ $1.24723 E+00$ I. $30034 E+00$ 1. $4423 E+00$ I.

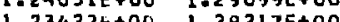
$1.23422 E+00$ I.R.217E+00 $1.22034 E+00$ 1.2J38SE+00 $1.22205 E+00$ 1.26603E+00 $1.2176 E+O C$ 1.25A7OE+ 00 $1.21305 t+U O \quad 1.25183 E+00$ 1.2087IE +00 1.2454LE+00 $1.20472 \tau+00 \quad 1.23944 E+00$ $1.201 C E E+00$ 1.23390E+ 00 $1.1977 T E+00 \quad 1.22877 E+00$ $1.18479 t+00 \quad 1.22405 E+00$ $1.19212 F+00 \quad 1.21972 E+00$ $1.18976 t+00 \quad 1.21576 E+00$ $1.18769 E+00 \quad 1.21217 E+00$ $1.18541 E+00$ 1.2CB94E+00 $1.183165+00$ 1.2034

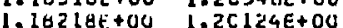
$1.18144<+00$ l. $199315+00$ la

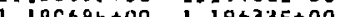
1. $18 C 64 E+00$ 1.1952 1. $100815+00$ 1.19526E+UO $1.10118+00$ 1.19445E+00 $1.29390 \mathrm{E}+00$ 1. $193 \mathrm{HOE}+00$ $1.18251 E+U O \quad 1.19354 E+00$ 1. $19370 E+D 0$ 
JAMMA $=1+(1-h)(12 S A /(1-h))(H h[G / G H C)-1)(11-h)$ HHC/RrCG)\#\#+ +W(2SH-2)

$$
S H=1.30 \quad R H C G=2.50 \quad S W=1.20
$$

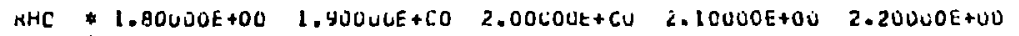

$1.00000 E-02 \div 1.44631 E+00$ $2.00000 t-02 * 1.46818 t+00$ $3.00000 k-02 \$ 1.42014 k+10$ $4.00000 k-02+1.43426 t+00$ $5.00000 t-02+1.41842 t+0$ $6.00000 t-0 ? \$ 1.40331 E+00$ 7.00000 $t-0 ? * 1.38690 t+00$ $8.00000 t-0$ ? 1.37 SIYt +00 $9.00000 t-02+1.36215 t+00$ $1.00000 \mathrm{E}-01+1.34476[+0 \mathrm{~L}$ $1.10000 E-01 \$ 1.33601 t+00$ $1.20000 t-01+1.32668 E+00$ $1.20000-01$ 1. $32608 E+00$ $1.30000 \mathrm{E}-1.31635 \mathrm{E}+0$ $1.40000 t-01 * 1.30642 t+0$ $1.50000 \mathrm{E}-01$ 2.29105E+0 $1.30000 E-01$ H. $1.28824 E+00$ 1.70000 E-01 1.27997E+00 $1.80000 t-01 * 1.27222 t+00$ 1.90000 E-OL \$ l.26498E+00 $2.00000 t-01$ 1.25823E+0O 2.10000E-01 1.25196L+00 $2.20000 \mathrm{t}=01+1.24616 \mathrm{bE}+0 \mathrm{C}$ $2.30000 E-01 * 1.24080 t+00$ $2.40000 t-01 * 1.23588 k+00$ $2.50000 t-02+1.23137 t+00$ $2.60000 t-01 * 1.22727 E+00$ $2.70000[-01+1.22357 \mathrm{E}+00$ $2.80000[-01 * 1.22024 t+00$ $2.90000 E-01 * 1.21728 t+00$ $3.00000 t-01 * 1.31467 E 00$ $3.10000 \mathrm{E}-01$ * $1.21240 \mathrm{E}+00$ $3.20000 \mathrm{E}-01$ 1.21046t+00 H. $1.208035+00$ 3.30000E-OL 1.208Ez+0O 3.40000E-OL 1.20751E+UU $3.50000 t-01 * 1.20648 t+00$
$3.00000 t-01 * 1.20313 t+00$

$1.5 .5019 E+C O$ $.56747 E+C O$ $1.54506 t+C O$ $1.5248 U E+C O$ . $544 \Delta 1 t+C 0$ $.4 a b c 8 E+C C$ $1.40741 E+C 0$ $1.444 Y 6 E+C O$ $1.43311 E+C O$ $1.41745 E+C O$ $1.40236 t+C O$ $38 B C$ It $+C D$ 1. 38BC It $+C 0$ $.31419 E+C$ . 3614 甘k $+C$ $1.33769 E+C O$ - 32678 1. $3267 a t+c 0$ - $31650 E+C O$ - 306 $64 E+C O$ $1.24777 E+C 0$ -2942 TE +C $1.28134 E+C O$ $1.27354 E+C O$ $1.267 \mathrm{C} B E+C$ $-26072 \mathrm{E}+\mathrm{CO}$ $1.25465 E+C 0$ 1. $24945 t+00$ $1.24451 E+C O$ I. $240 U 2 E+C$ $1.24002 E+C O$
$1.2354 G E+C$ $1.23596 E+C O$
$1.23230 E+C O$ $1.23230 E+C O$ $1.229 \mathrm{CSE}+\mathrm{C}$ $1.22618 E+C$ $1.22307 E+C 0$
$1.22152 E+C 0$ $1.21911 E+C O$ 1.70t $26 t+50$ $1.68 c 37 E+C u$ $.65357 i+C j$ $1.62785 t+C 0$ $1.603 L 2 t+C O$ l. $27552 L+C C$ 1. $55687 E+C C$ $1.53521 \mathrm{E}+\mathrm{CO}$ $1.51449 E+C O$ $1.49471 \mathrm{t}+\mathrm{CD}$ $1.47583 t+C O$ $1.45784 t+C O$
$1.44 C 71 t+C D$ $1.42442 E+C U$ $1.42442 E+C O$ $1.40894 t+C O$ $1.34427 k+C 0$ $1.30036 r+C 0$ $1.367212+c u$ 1. $35478 E+C O$ 1. $34307 t+C U$ $1.33204 t+00$ $1.3216 \mathrm{gE}+\mathrm{CU}$ $1.31197 L+C O$ $1.30288 E+C 0$ 1. $29440 t+00$ $1.28651 \mathrm{E}+\mathrm{CU}$ $1.2741 \theta E+C U$ $1.27241 E+C O$ $1.26617 E+C O$ $1.26 \mathrm{GA} 4 \mathrm{H}+\mathrm{CU}$ $1.2552 C E+C O$ $1.2552 \mathrm{CE}+\mathrm{CU}$ $1.25 C 44 E+C 0$ 1.24614E+CU $1.24228 E+C O$
$1.23885 E+C O$ $1.23583 E+C D$ 1. 807 thE+00 1. $17224 E+00$ 1. $74411 E+00$ $1.71421 t+u$ l. 6 f5jatt+Ja $1.657916+0$ $1.63157 \mathrm{E}+0$ $1.60630 t+00$ 1. $54211 \mathrm{t}+00$ 1.55899k+OC $1.536 \rightarrow 1 E+00$ 1 - $15 B 4 E+U U$ I. $49576 E+U$ 1. $42477 \mathrm{E}+00$ 1. $40424 E+00$ $1.40424 \mathrm{E}+00$ I. $39454 E+00$ 1. $38065 E+00$ 1. $36755 E+U O$ L. $35522 E+00$ $1.34363 E+00$ 1. $3.3275 E+00$ 1. $32257 E+00$ 1. $31306 E+00$ 1. $30421 E+00$ 1. $2959 B t+0 \mathrm{C}$ 1. $28036 E+00$ $1.28836 E+00$ 1.28 $27486 t+00$ $1.27486 \mathrm{t}+00$ 1. $26894 t+00$ $1.26354 \mathrm{E}+00$
$1.25865 \mathrm{E}+00$ $1.25865 \mathrm{E}+00$
$1.25425 \mathrm{E}+00$

\section{$1.98494 l+00$} $1.94991 t+00$ 1. $91136 t+00$ $.874251 .+00$ $.83435 t+00$ $1=80424 t+00$ $1.77129 t+00$ $1.73965 t+00$ $1.70431 t+0 u$ $1.68024 t+00$ $1.05240 t+00$ $.625761+00$ $.62576 \mathrm{t}+00$ $1.60630 E+0$ . $57359 E+U 0$ - $55280 \mathrm{r}+0$ $.50404 E+00$ $.44563 k+0 C$ $1.47062 E+00$ $1.45258 E+00$ $1.43549 E+00$ $1.41933 \mathrm{E}+0 \mathrm{O}$ $1.40405 t+0$ $1.36965 E+00$ 1. 3760BE+UO 1. $36333 E+00$ . $35137 E+0$ $.34017 E+00$ l.32971t+0 - $31997 \mathrm{E}+0 \mathrm{O}$ $1.310418+00$ $1.30252 E+00$ $1.294776+00$ $1.28765 E+00$ 1.2811 IE +00 $1.27515 t+00$
$2.15482 t+C 0$ $2.10782 t+00$ $.00250 \mathrm{E}+\mathrm{CO}$ $2 . U 1683 F+C O$ $1.9707 E r+C 0$ $1.936316+C L$ $1.69738 r+00$ 1. $65496 t+C 0$ - B240t + CO $1.756 .55 \mathrm{t}+\mathrm{CO}$ $1.7248 \mathrm{t} \mathrm{L}+00$ $1.63457 E+C O$ $1.065586+C O$ $1033885+00$ $1.61144 t+C 0$ .5 $16+00$ 50 $105+00$ 1. DI - - $11=7 E+00$ $1.47735 E+00$ $1.45880 t+00$ $1.44127[+00$ $1.4247 \mathrm{IE}+\mathrm{CO}$ $1.4 C 4 C 9 E+C O$ I. $39439 t+00$ 1. $3805 B E+00$ $1.36763 t+00$ $1.35551 E+00$ 1. $34419 E+00$ $134195+00$ $1.33365 \mathrm{E}+\mathrm{CO}$ $1.323 B 6 E+C O$ $1.31479 E+00$ $1.30041 E+00$
$2.336425+00$ 2. $28184 k+00$ $22916 E+00$ $1 / 835 E+00$ $2.12437 t+00$ 2. $082 \mathrm{LaF}+0$ $? .03675 t+00$ $1.99305 E+00$ - . MlOze + 1. $91066 E+00$ $1.87190 t+00$ I. $83473 E+00$ TU 7 ICE + OO 1. $76497 E+D 0$ $1.73732 E+00$ $1.70110 \mathrm{E}+00$ - 70110E +OQ $64203 E+100$ . 1. $1.54195 E+00$ 1. $51980 E+00$ 1. $49 B$ BCE + OO $1.47892 E+U O$ $1.16013 \mathrm{E}+U 0$ $1.44240 E+00$ $1.42570 E+O D$ $1.40998 \mathrm{dE}+00$ $1.39522 E+00$ $1.38139 E+00$ $1.35639 E+U 0$ $1.345165+00$ 1. $34735+00$ 1. $32506 \mathrm{E}+00$

$2.53509 E+00$ $2.47235 E+00$ $41174 E+00$ . $35321 E+00$ $2.24674 E+00$
$2.24228 E+00$ $2.16980 E+00$ $2.13926 E+00$ $2.09061 E+00$ $2.04383 E+00$ $1.49887 E+U O$ $1.95570 E+00$ $1.91427 E+00$ $.87454 E+00$ . $83648 E+04$ LCOO5E+00 1.76521E+00 $70013 E+00$ 
GAMIIA $=1+(1-W)(2 S R /(1-h))(R H C G / A H C)-1)(11-W) A H C / R R C G)+\$ 5+W(2 S H-2)$
Sk $=1.30$
AHCG $=2.60$
$5 H=2.20$

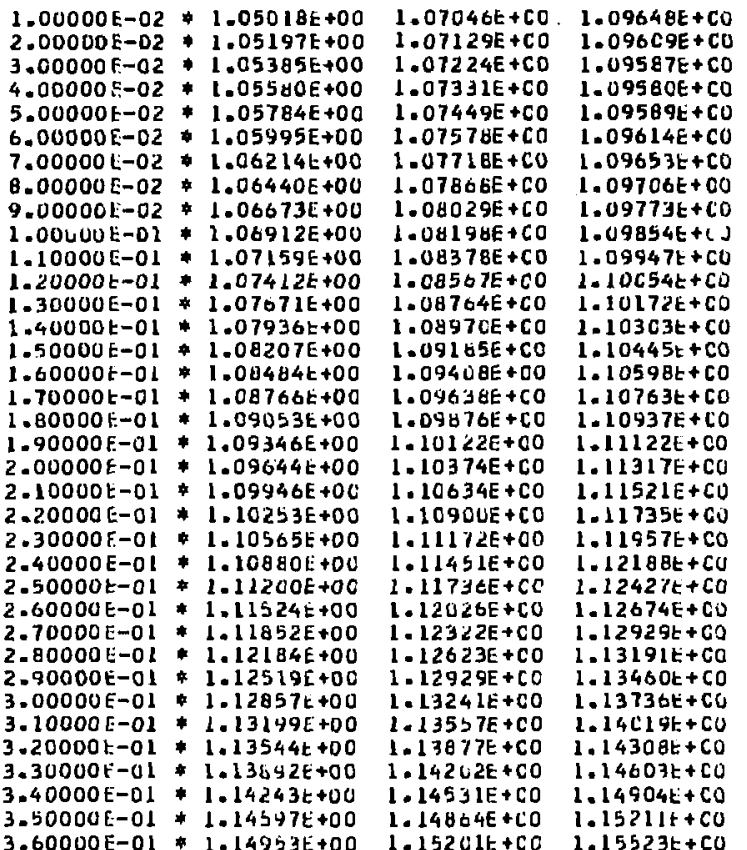

\section{$1.12911 E+00$}

1. $12720 E+00$

$1.12551 E+00$

1. $12404 E+00$

$1.12277 E+00$

1. $12170 E+00$

$1.12083 E+00$

$1.12015 t+00$

$1.11906 \mathrm{E}+00$

1. $11935 E+00$

$1.11921 E+0$

$1.11944 E+00$

$1.11944 E+0$

$1.11940 E+00$

$1.12032 E+0$

1. $12098 E+00$

$1.12179 \mathrm{E}+00$

$1.12275 E+00$

$1.123 \forall 3 E+00$

1. $12506 \mathrm{E}+00$

$1.12641 \mathrm{E}+00$

1. $1278 B E+00$

1. $12947 E+00$

$1.13118 E+00$

$1.13300 E+O 0$

$1.13493 \mathrm{E}+\mathrm{U}$

1. $13646 E+0$

$1.14132 \mathrm{E}+00$

1. $14132 E+0$

$114363 E+00$

$1.14854 \mathrm{E}+00$

1. $15111 E+00$

$1.15111 E+00$
$1.15377 E+00$

1. $1565 U E+00$

1. $1593 \cup E+00$

\section{$.16924 E+00$}

$1.16547 t+00$

$1.16199 \mathrm{E}+00$

1. $15878 E+00$

$1.15545 t+00$

$1.15077 E+00$

$1.14861 E+00$

$1-14669 t+00$

$1.14501 t+00$

$1.14355 t+00$

$1.14232 t+00$

$1.14131 E+C 0$

- $14051 t+00$

$1.13991 E+00$

- $13451 E+00$

- $13930 E+U 0$

$1.13 \times 27 E+00$

1. $13943 E+00$

1.13476E+UD

$1.14025 \mathrm{E}+00$

$1.14091 E+00$

$1.14 k 73 z+00$

$1.14270 t+00$

$1.14381 E+00$

$14307 E+00$

.14646E+00

$1.14799 t+00$

$1.14404 t+00$

-15r41e+00

$1.15330 E+00$

1.1550 .

- $15742 \mathrm{c}$

$1.16195 t+00$

$1.16436 t+00$
1. $50000 E+00$

$1.600 C C E+00$

1.7COONE+0O

$.21775 E+00$

1. $26587 E+00$

1. $25869 \mathrm{E}+00$

1. $25095 t+00$

$1.24365 t+0 C$

$1.23677 t+00$

$1.233030 E+00$

$1.22424 E+00$

1.21E57t+00

$1.21328 E+00$

$1.20835 t+00$

$1.20379 E+00$

$1.199500+00$ 1.1957Cl+ou 1.1921ectoo $1.18894 E+00$ 1. $146 C 25+100$ $1.18341 E+00$ 1. $181095+00$ $1.17905 E+00$ $1.17128 \mathrm{E}+00$ $1.17578 t+00$ 1. $17453 E+00$ $1.11354 t+00$ i. $1354 \mathrm{E}+00$ $1.1727 k+0$ 1.1725e+0 $1.17194 t+00$ 1. $17185 E+0$ $1.17197 k+00$ 1.17225e+00 ..17295e+00 $1.17456+00$ $\begin{array}{ll}1.16511 E+C 0 & 1.174361+00 \\ 1.16679 E+C 0 & 1.17540 E+00 \\ 1.168605+00 & 1.17661 E+00\end{array}$ $\begin{array}{ll}1.16511 E+C 0 & 1.174361+00 \\ 1.16679 E+C 0 & 1.17540 E+00 \\ 1.168605+00 & 1.17661 E+00\end{array}$ $\begin{array}{ll}1.16511 E+C 0 & 1.174361+00 \\ 1.16679 E+C 0 & 1.17540 E+00 \\ 1.168605+00 & 1.17661 E+00\end{array}$ $1.34340 E+00$ 1. $33168 E+00$ $1.12053 E+00$ $1.30992 E+00$ $1.25985 E+00$ $1.29031 E+00$ $1.28127 E+00$ 1.26458E+00 1.26468E+00 . .. 1. $1.23703 E+00$ $1.23120 E+00$ $1.22574 E+00$ $1.22075 \mathrm{E}+00$ 1.21611E+00 C. $21185 E+00$ $1.20794 E+00$ $1.20439 E+00$ $1.20117 E+00$ $1.15829 E+00$ $1.17572 E+00$ $1.19346 E+0 D$ .19346E+00 1. 1 1 $90 E+00$ $1.18982 E+00$ $1033+\infty 0$ 1. . $1.18581 E+00$ 1. $12543 \mathrm{E}+00$ . $1852 A E+C 0$ l.18535t +00 $1.18563 E+00$ $1.18612 E+00$ 1. $18681 E+00$ 
GAMMA $=1+(2-W)(12 S R /(1-W))(R H E G / R H C)-()((1-W) R H C / R T C G)+5+W(25 W-2)$

$$
S H=1.3 C \quad R H C G=2.60 \quad S W=1.20
$$

AHO $\quad 1.80000 E+0 D \quad 1.90000 E+C O \quad 2.00000 E+C O \quad 2.10000 E+00 \quad 2.20000 E+00 \quad 2.30000 E+C 0 \quad 2.40000 E+00 \quad 2.50000 E+00$

$1.00000 E-02 * 1.42227 E+00$ $1.00000 E-02 * 1.42227 E+00$
$2.00000 E-02 * 1.40700 E+00$ 3.00000 E-02 $+1.39242 E+0$ 4.00000E-02 1.37851E+0 $5.00000 E-02 * 1.36525 E+00$ $6.00000 \mathrm{E}-02+1.35262 \mathrm{t}+00$ 7.00000E-O2 *1.34062E+00 $8.00000 \mathrm{E}-02 \div 1.32422 \mathrm{E}+00$ $1.00000 \mathrm{E}-01 * 1.30816 \mathrm{E}+00$ $1.10000 E-01 * 1.2984 B E+00$ $1.10000 E-01 * 1.29848 E+00$
$1.20000 t-01 * 1.28934 t+00$ $1.30000 E-01 * 1.28073 E+00$ $1.40000 \mathrm{E}-01 * 1.27263 \mathrm{E}+00$ $1.50000 \mathrm{E}-01 * 1.26503 E+00$ $1.60000 \mathrm{E}-01 \quad 1.25792 \mathrm{E}+00$ $1.70000 \mathrm{E}-01 * 1.25127 \mathrm{E}+00$ $1.80000 E-01 * 1.245085+00$ $1.90000 E-01$ 1.23034E+0 1.9000 E $2.00000 E-01$ 1.23402E+0 .10000E-OL H 1.22912E+0 $2.20000 E-O 1+1.22463 E+00$ $2.30000 E-O 1+1.22052 E+00$ $2.40000 \mathrm{E}-01+1.21679 E+00$ $2.50000 E-01 * 1.21343 E+00$ $2.60000 E-01 \div 1.21042 \mathrm{E}+00$ 2.700 OE-0I 1.20775E+0O $2.80000 E-01+1.20541 E+00$ * 1.20339E+00 . 1.203 . 1.20167E+0 . . 20000E-O1 1.19912E+00 .30000E- 1.19825et00 $3.40000 E-01$ *1.19765E+0O $3.50000 E-01 * 1.19731 E+00$
$3.60000 \mathrm{E}-01 * 1.19720 \mathrm{E}+00$

$1.51293 \mathrm{HE}+\mathrm{CO}$ $1.44362 \mathrm{CE}+\mathrm{CO}$ $1.47513 E+C O$ $1.45745 \mathrm{E}+\mathrm{CO}$ $1.44054 E+C O$ $1.42440 E+C O$ $1.40900 E+C 0$ L. $39432 E+C O$ 1. $38036 E+C D$ $1.38036 E+C D$ $1.35447 \mathrm{E}+00$ $1.34252 E+C O$ $1.33120 E+C D$ 1. $32050 E+C O$ 1. $31040 E+00$ 1. $300 \mathrm{BBE}+\mathrm{CD}$ $1.29193 \mathrm{E}+\mathrm{CD}$ $1.28354 E+00$ $1.28354 E+00$ $1.27568 E+00$ $1.26833 E+C O$ $1.26150 E+C D$ $1.25514 E+00$ $1.24926 E+00$ $1.24384 E+C O$ $1.23886 E+00$ $1.23431 E+00$ 1. $23017 E+C O$ 1. $22644 E+C D$ $1.22308 E+00$ $1.2230 E+00$ - $22010 E+00$ $1.21748 E+C 0$ $1.21520 E+C 0$ $1.21326 E+00$ $1.21163 E+00$ $1.21031 E+C 0$ $1.20929 E+00$
$1.61615 E+C O$ $1.59229 \mathrm{k}+\mathrm{CO}$ $1.56934 \mathrm{E}+\mathrm{CU}$ $.54744 E+C O$
$1.52642 E+C O$ 1. $50629 E+C O$ $1.48705 E+00$ $1.46867 E+C O$ $1.45113 E+C O$ $1.43441 E+00$ $1.41848 t+00$ $1.40333 E+C O$ 1. $38 B 94 E+C O$ 1. $3752 \mathrm{Bt}+\mathrm{CO}$ $1.36234 E+00$ 1. $3501 \mathrm{OE}+\mathrm{CO}$ $1.33853 k+C O$ $1.32762 E+C O$ $1.31735 E+0$ $1.29864 t+D U$ $1.29018 \mathrm{E}+00$ $1.29018 E+00$ $1.28227 E+C O$
$1.27492 E+C O$ $1.26809 E+00$ $1.26178 \mathrm{E}+\mathrm{CO}$ L. $25596 E+00$ $1.25062 E+C O$ $1.24574 E+00$ $1.24131 E+00$ 1. $23731 \mathrm{E}+00$ $1.23373 E+C$ $1.23054 E+00$ $1.22774 \mathrm{E}+\mathrm{CO}$ $1.22531 E+C O$ $1.22324 E+C O$
1. $73206 E+00$ 1. $70370 \mathrm{OE}+00$ $1.6758 \mathrm{EE}+0$ $1.64916 E+00$ h. $62352 E+00$ 1. $59894 E+00$ 1. $57539 E+00$ 1.552 y $5 E+00$ 1.531 $305+00$ $1.51071 \mathrm{E}+00$ $1.49105 E+00$ $1.47231 E+00$ $1.45446 \mathrm{E}+0 \mathrm{O}$ $1.43747 \mathrm{E}+00$ $1.42133 \mathrm{E}+00$ $1.40600 E+00$ 1. $39148 E+00$ 1. $37773 \mathrm{E}+00$ 1. $36474 \mathrm{E}+00$ 1. $3544 \mathrm{E}$ $1.35247 \mathrm{E}+00$ $1.34092 \mathrm{E}+00$ 1. $33006 \mathrm{E}+00$ 2.31987E+00 1. $3 \angle 032 E+00$ 1. $30140 E+00$ $1.29309 E+00$ 1. $28537 \mathrm{E}+00$ $1.278 \times 1 E+00$ 1.27821E+00 $1.27161 \mathrm{E}+00$ $1.25997 E+00$ $1.25490 E+00$ 1. $25490 E+00$ $1.25030 \mathrm{E}+00$ $1.24616 E+00$ $1.24246 E+00$ $1.23919 E+00$
$1.86312 E+00$ $1.82852 \mathrm{E}+00$ $1.79523 E+00$ $1.76322 \mathrm{t}+00$ $1.73246 E+00$ $.67459 E+00$ $1.64742 E+00$ $1.62140 E+00$ $1.62140 E+00$ $1.57268 E+00$ $1.54993 E+00$ $1.52822 E+00$ $1.50751 E+00$ $1.48779 E+00$ $1.46402 E+00$ $1.45119 E+U D$ $43426 E+00$ $41822 \mathrm{E}+00$ $1.41822 E+00$ $1.40303 E+00$ 1. $38867 E+00$ $.37513 E+00$
$.36236 t+00$ 1. $36236 t+00$ $1.35035 \mathrm{E}+00$ $1.33908 E+00$ $1.32853 E+00$ $1.31866 \mathrm{t}+00$ 1. $30946 E+00$ 1. $30091 E+00$ -29298E+00 $1.28565 e+00$ 1.27890E+OD $1.27271 \mathrm{t}+00$ $1.26706 E+00$ $1.26193 E+00$
$1.25730 E+00$
$2.00813 t+00$ $1.96733 \mathrm{~F}+0 \mathrm{U}$ 1. $92802 E+00$ $1.8901 \mathrm{BE}+C \mathrm{CO}$ 1. $6597 \mathrm{BE}+\mathrm{CO}$

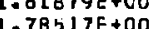
$1.75290 E+C O$ $1.72195 E+00$ $1.69227 E+00$ $1.66386 \mathrm{E}+00$ $1.63667 E+00$ $1.61067 E+00$ 1. $58584 F+00$ $1.562 \angle 5 E+00$ $1.53956 E+C O$ . $51806 E+O$ - $49760 \mathrm{OE}+\mathrm{CO}$ $1=47816 E+00$ $1.45972 E+00$ $1.44224 E+00$ $1.42565 E+00$ $1.41006 \mathrm{t}+00$ $1.39531 \mathrm{E}+00$ $1.38142 \mathrm{E}+\mathrm{CO}$ 1. $36835 \mathrm{E}+00$ 1. $35609 \varepsilon+00$ $1.34460 E+00$ 1. $33387 \mathrm{E}+00$ $132386 E+00$ $1.31456 E+00$ 1. $30593 E+C O$ $1.329796 \mathrm{E}+00$ $1.29062 \mathrm{E}+00$ 1.2838 BE +0 1. $27773 \mathrm{E}+00$
$2.16814 E+U 0$ $2.12063 \mathrm{~L}+00$ 2.0747Ut +00 2. $03 C 5 E E+00$ $1.98800 t+00$ $1.94703 E+00$ $1.90763 E+00$ $1.86976 E+00$ 1. $83339 \mathrm{E}+0 \mathrm{D}$ 1. $79849 E+00$ $1.76502 E+00$ $1.73295 E+00$ $1.70225 E+00$ $1.672 \mathrm{BUE}+00$ $1.64481 \mathrm{~L}+00$ 1.61801E+DO $1.59245 E+00$ $1.56809 t+00$ $1.54491 t+00$ 1.52287E+0 $1.52287 E+00$ $1.48208 E+00$ $1.46327 E+00$ $1.44548 E+00$ $1.42867 E+00$ $1.41283 \mathrm{E}+0 \mathrm{O}$ $1.39791 \mathrm{E}+00$ 1. $38388 E+00$ 1. $37073 E+00$ I. 3584 1E+00 $1.34691 \mathrm{E}+0 \mathrm{C}$ 1. $33620 \mathrm{E}+00$ - $32624 t+00$ 1. $31701 E+00$ 1. $30848 \mathrm{E}+00$ 1. $30063 E+00$

2. $34374 E+00$ 2. $28886 \mathrm{E}+00$ 2. $23589 E+00$ $2.18479 E+00$ 2. $13553 E+00$ $2.08803 E+00$ $2.04238 E+00$ $1.99842 E+00$ $1.95616 \mathrm{E}+00$ $1.91555 E+00$ $1.87657 \mathrm{E}+00$ 1. $83917 E+00$ 1. $80333 \mathrm{~J}+00$ 1. $76899 \mathrm{E}+00$ 1. $73614 E+40$ 1. $70473 E+00$ $1.67473 \mathrm{E}+00$ $1.64809 E+00$ $1.61880 E+00$ 1.59280E+00 1. $54457 \mathrm{E}+00$ $1.52227 E+00$ $1.50113 E+00$ $1.48112 E+00$ $1.46221 E+00$ $1.44435 E+00$ 


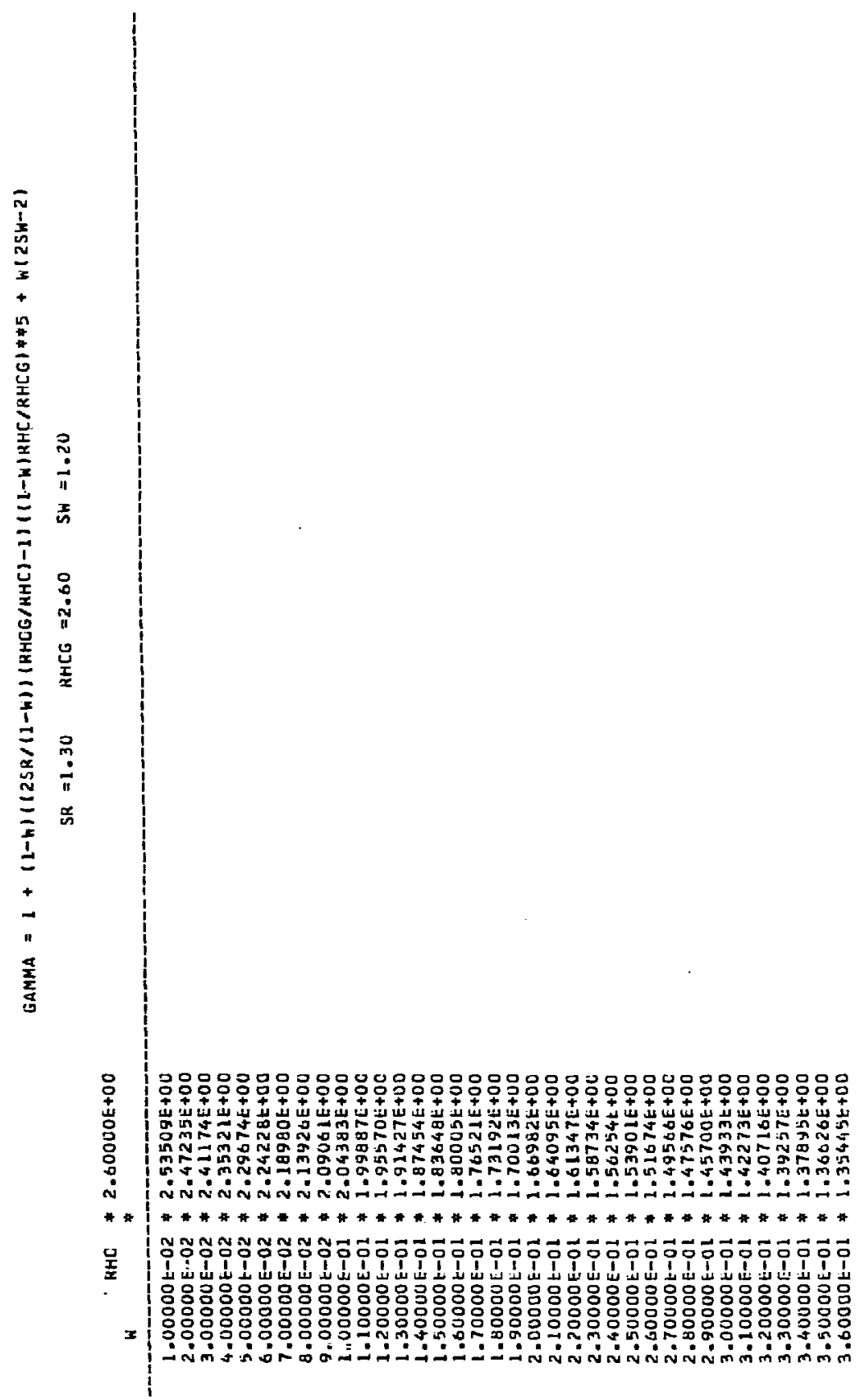


LAMMA $=1+(1-h)((25 R /(1-h))(R H C G / R H[)-1)(1-W) R H C / R+C G)+5+W(25-2-2)$

$$
S R=1.30 \quad R H C G=2.65 \quad S W=1.20
$$

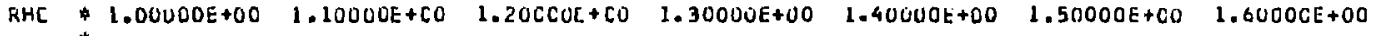

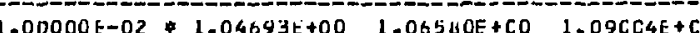

1. $1.04880 E+001.06406 E+C O$

$1 .-120475+00$

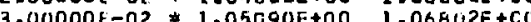

$1.08995 \mathrm{E}+\mathrm{CJ}$

1.15786E+00

$1.20312 \mathrm{E}+00$

$1.25704 E+00$

$1.24924 E+00$
$1.24187 E+00$

1. $32047 E+00$

$1.06802 E+C 0$

$1.29063 E+00$

1. $06929 E+C O \quad 1.09 C 24 E+C O$

$1.11704 E+00$ 1.15164E+UO

$1.19280 E+00 \quad 1.24187 E+00$

$1.29963 E+00$

$1.09 C 6 U E+[U$ 1.11563E+00 $1.14647 E+00 \quad 1.183 E 1 E+00 \quad 1.22 E 37 E+00 \quad 1.28086 E+00$

$1.09110 E+C O \quad 1.11491 E+00 \quad 1.14425 E+00 \quad 1.179 B C E+C O \quad 1.22222 E+00 \quad 1.27221 E+00$

$1.01313 E+C O \quad 1.09174 t+C O$

$1.0754 \mathrm{UE}+\mathrm{CO} \quad 1.09251 E+C O$

$609 E+00$

$1.114 U 2 E+00 \quad 1.14053 \mathrm{E}+00 \quad 1.17268 \mathrm{E}+00$

$1.27271 E+00$

$7.00000 \mathrm{E}-02 \div 1.05973 \mathrm{E}+00$

$8.00000 \mathrm{E}-0$ ? $\$ 1.06211 \mathrm{E}+00$

9.0000

$1.04341 E+C$

(2)

$1.216465+00$

$1.26404 E+00$

$1.09444 E+C O \quad 1.11382 E+00 \quad 1.13 / 72 E+00 \quad 1.16672 E+00$

$1.20605 E+00$

$1.25634 E+00$
$1.24909 E+00$

1. OHOSUE +C

$1.09558 t+C Q$

$1.13664 \mathrm{E}+00$

$1.2422 \mathrm{BE}+00$

$1.10000 E-01 * 1.06944 E+00$

$1.20006 L-01 * 1.07227 E+00$

1. OE $30 \perp E+C O$

$1.09685 t+C O$

$1.11427 E+00$ 1.13577E+00 $1.16187 E+00$

$1.19706 t+00$

$1.23589 E+00$

$1.08513 E+C O \quad 1.09823 E+C O$

$1.15483 t+C 0$

$1.19308 E+00$

$1.22992 E+0 D$

$1.09973 E+C O \quad 1.143 E+00$ 1.13510E+00 $1.15983 E+C L$

$18942 E+00$

$1.22436 E+00$

$1.40000 \mathrm{E}=01$ \&.07771E+0C 1.03733E+CO

$1.50000 \mathrm{E}-01 * 1.08051 \mathrm{E}+00$

$1.0090 C E+C O$ I.10133E+CO

$1.13463 t+00$

$1.1585 \mathrm{CE}+\mathrm{CO}$

$C B E+00$

1. $21919 \mathrm{E}+00$

$1.10133 E+C 0$

$1.1160 \mathrm{O}$

$1.15651 E+00$

$18305 E+00$

$1.21440 \mathrm{E}+00$

$1.09155 E+C 0$

$1.103 C 3 E+C 0$ 1.11699E+00 $1.13425 E+00$

$15521 E+00$

$100310+40$

$1.21440 \mathrm{E}+00$

$1.09647 E+C O$

$1.106745+C U$ I $11919 E+00$

$1.13432 E+00$

$1.15414 E+00$

$1.12048 \mathrm{E}+00 \quad 1.13500 \mathrm{E}+00$ 1.15265E+CU

$1.15265 \mathrm{E}+\mathrm{CU}$
$1.15223 \mathrm{E}+00$

$1.12189 \mathrm{E}+00 \quad 1.13558 \mathrm{E}+00$

l. $10206 E+C D$ 1. $10803 E+C O$

$1.11083 E+C 0$

$1.11301 E+C O$

$343 E+0 U$ L $1.13632 t+0$

$1.15197 \mathrm{E}+00$

$1.13721 E+D D$

$1.12684 E+00 \quad 1.13824 E+00$

1. $10731 E+C$

1. $11033 E+00$

1.11320E +CO

$1.11762 \mathrm{E}+\mathrm{CO}$

1. $15213 \mathrm{E}+\mathrm{CO}$

$1.12005 E+C O$

$1.13942 E+00$

$1.15248 E+C 0$

1. $1.593 \mathrm{E}+00$

$1.17381 E+00$ 1. $20222 E+00$

$17210 E+00$ l. $19501 E+00$ 1.1700 +00 1.1980 160800000

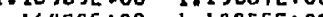
$1.13068 E+00$ 1.14074E+DO $1.15300 E+00$ E $1.16815 E+00$ $5000 \mathrm{E}-01$. $11115 \mathrm{E}+0 \mathrm{O}$ $2.60000 E-01 * 1.11444 E+00 \quad 1.11910 E+C O$ $2.70000 E-01 * 1.11777 E+00$ $2.80000 \mathrm{E}-01+1.12214 \mathrm{E}+00$ $1.12 B 35 E+C D$ $3.00000 E-01+1.12796 E+00$ $3.10000 k-01+1.131425+00$ $3.20000 \mathrm{E}-01 * 1.13491 \mathrm{E}+00$ $3.30000 E-01 * 1.13843 E+00$ $3.40000-01+1.14197 E+00$

$3.50000 \mathrm{E}-0 \mathrm{~L} * 1.14554 \mathrm{E}+00$
$3.60000 \mathrm{t}-0 \mathrm{~L} * 1.149 \mathrm{~L}+\mathrm{E}+00$ $1.12513 E+C D$

$1.13275 E+0 D \quad 1.14219 E+00$

$1.15368 \mathrm{E}+00$ $1.12778 E+C 0$ 1. $13492 E+00 \quad 1.14376 E+00 \quad 1+15454 E+00$ $1.13329 E+C O \quad 1.13953 E+00 \quad 1.14727 E+00 \quad 1.15671 F+00$ $1.13613 E+C O \quad 1.14197 E+00 \quad 1.14920 E+00 \quad 1.15802 E+00$ $1.13904 E+C O \quad 1.14448 E+00 \quad 1.15124 E+00 \quad 1.15947 E+00$ 1. $13474 \mathrm{E}+\mathrm{CO}$ 1. $13800 E+00$ $1.14131 E+C O$ $1.14465 E+C D$ $1.13904 E+C 0$ $1.14504 t+C 0$ $1.14812 E+C 0$ $1.14448 E+00$

$1.14708 E+00$ $1.15124 \mathrm{E}+00 \quad 1.15947 \mathrm{E}+00$ $1.15338 \mathrm{E}+00 \quad 1.16106 \mathrm{E}+00$

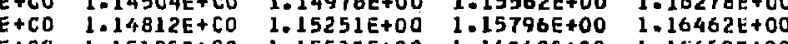

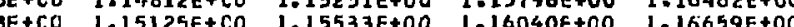
$\begin{array}{lllll}1.15125 E+C O & 1.15533 E+00 & 1.16040 E+00 & 1.16659 E+00 & 1.174 C 4 E+00 \\ 1.15443 E+0 D & 1.15822 E+00 & 1.16292 E+00 & 1.16867 E+00 & 1.17559 E+00\end{array}$

$1 E+00$ $16750 E+00$ $.16749 E+00$ $16768 t+00$ $1168 C 6 E+00$ $16863 E+00$ 1. $16938 E+00$ $1.17139 \mathrm{E}+00$ $1.17264 E+00$ - $17264 E+00$ 1. $18855 E+00$ $1.18671 E+00$ $1.18516 E+00$ $1.18387 \mathrm{E}+00$ $1.18264 E+00$ 1. 1 B207E+00 $1.28153 E+00$ $1.18123 E+00$ $1.18115 \mathrm{E}+00$ $1.18128 E+00$ $1.18162 \mathrm{E}+00$ $1.18216 E+00$

$\begin{array}{ll}174 C 4 E+00 & 1.18290 E+00 \\ 17559 E+00 & 1.18381 E+00\end{array}$ 
GAMMA $=1+(1-W)((2 S R /(1-h))(R H C G / R H C)-1)((1-W) H H C / R H C G)+\$ 5+W(25 W-2)$

$S R=1.30 \quad$ RHCG $=2.65 \quad S H=1.20$

RHC $\quad 1.80000 E+00 \quad 1.900 U D E+00 \quad 2.00000 E+C O \quad 2.10000 E+00 \quad 2.20000 E+C O \quad 2.30000 E+00 \quad 2.40000 E+00 \quad 2.50000 E+00$

\begin{tabular}{|c|c|c|c|c|c|c|c|c|c|}
\hline 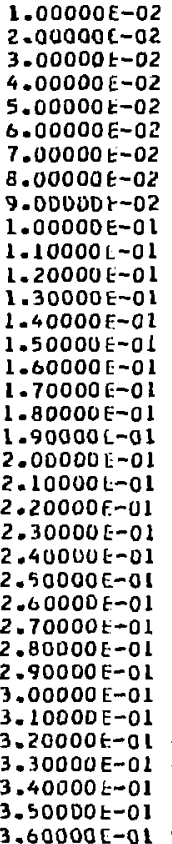 & * & $\begin{array}{l}+00 \\
+00 \\
+00 \\
+00 \\
+00 \\
+\infty 0 \\
+00 \\
+00 \\
+00 \\
+00 \\
+00 \\
+00 \\
+00 \\
+\infty 0 \\
+00 \\
+00 \\
+00 \\
+00 \\
+00 \\
+00 \\
+00 \\
+00 \\
+00 \\
+\infty 0 \\
+00 \\
+\infty 0\end{array}$ & 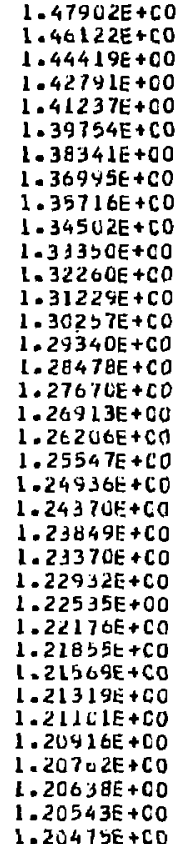 & 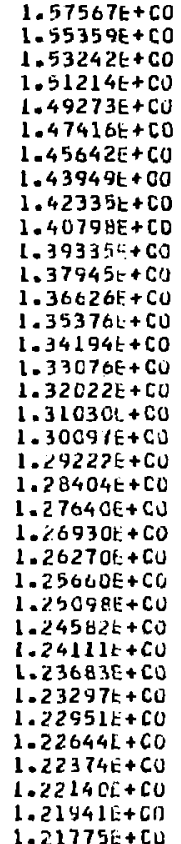 & $\begin{array}{l}1.65799 E+00 \\
1.6321 B E+00 \\
1.60741 E+00 \\
1.58366 E+00 \\
1.56091 E+00 \\
1.53912 E+00 \\
1.51829 E+00 \\
1.49831 E+00 \\
1.47937 E+00 \\
1.46124 E+00 \\
1.44397 E+00 \\
1.42754 E+00 \\
1.41191 E+00\end{array}$ & $\begin{array}{l}00 \\
00 \\
00 \\
00 \\
00 \\
00 \\
00 \\
00 \\
00 \\
0 \\
00 \\
00\end{array}$ & 1 & טנ ט & 1 \\
\hline
\end{tabular}

$3.60000 E-01+1.19349 t+00$

$1.2047 \mathrm{JE}+\mathrm{CD}$

. $21775 E+C$

$1.23262 \mathrm{E}+00 \quad 1.24931 \mathrm{E}+00$

$1.26995 E+00$ 


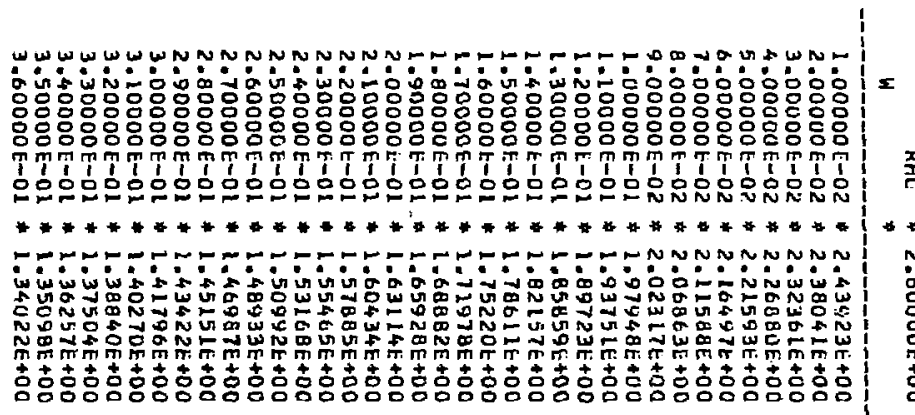


GAMMA $=1+(1-h)((2 S R /(1-h))($ HHCG/RHC $)-1)(11-h) K H(/ R H C G) * \$ 5+W(2 S W-2)$

$$
S R=1.3 \mathrm{C} \quad \mathrm{RHCG}=2.70 \quad S H=1.20
$$

1. $10 \mathrm{CLE}+\mathrm{CO}$

$1.00000 \mathrm{E}-02 * 1.04346 \mathrm{E}+00$

$2.00000[-02 \div 1.04605 \mathrm{E}+00$

1. $30000 E+00$

AJUODE+00 1.50000E+00

1. $6000 C E+O 0$ 1.7COOOE+OO

$2.00000 \mathrm{C}-02.1 .04605 \mathrm{E}+00$

$3.00000 E-02 \$ 1.04821 E+0 C$
$4.00000 r-02 * 1.05444 E+00$

$5.000005-02 * 1.05273 t+00$

$6.00000 \mathrm{E}-02 * 1.05 \mathrm{bl0t}+00$

7.00000t-02*1.05753t+00

$8.00000 \mathrm{E}-02 * 1.000 \mathrm{C} 2 \mathrm{E}+00$

$9.00000 E-02 * 1.06237 E+00$

$1.00000 t-01 * 1.06519 t+00$

$1.100001-01+1.067266+00$

$1.20000 E-01 * 1.0705 \& E+00$

$1.30000 E-01 * 1.07336 t+00$

$1.40000 z-0 l$ \& $1.07 a z 0 E+00$

$1.50000 t-01 * 1.07908 t+00$

$1.60000 t-01$. 1.CazLlt to

$1.70000 r-01+1.08499 t+00$

1.B0000[-01 1.0B802t+0L

$1.90000 \mathrm{C}-01 \$ 1.09169 E+0 C$

$2.00000 \mathrm{r}-01+1.094201 .+0$

$2.100001 .01 * 1.077301 .+00$

$2.20000 t-01 \% 1.10056 t+0 C$

$2.300006-01 * 1.103796 .+0 \mathrm{C}$

$2.40000 \mathrm{r}-01$ 1.107L6rtou

$2.50000 \mathrm{f}-01 * 1.11037 t+00$

$2.60000 \mathrm{r}-01.1 .113716+00$

$2.70000 \mathrm{t}-01 * 1.11709 \mathrm{t}+00$

2. B000OL-UL $\$ 1.12050 \mathrm{~L}+00$

$2.40000 t-01+1.12343 t+00$

$3.00000 t-01+1.12740 t+00$

3.:0000E-01 1.1304Ut+00

$3.20000 t-01+1.134 \% 2 t+00$

$3.30000 t-01 * 1.13791++00$

$3.40000 t-01 * 1.14155 t+00$

$3.400001-01$.
$3.50006 E-01$ 1.14S1SE+00

$3.5000 L E-01$ 1.14bIbL+OL
$3.60000 E-01 * 1.14877 E+0 C$

-062 ECE +CO

1. $U 65 C 2 E+C O$

. $06502 E+C 0$

1. $04717 \mathrm{t}+\mathrm{C}$

$1.0705 \% \mathrm{E}+\mathrm{CO}$

$.0724 \mathrm{LE}+C O$

$.014 J 3 E+C D$

$.076 J 3 E+C O$

$.01142 E+C O$

$.00059 \mathrm{E}+\mathrm{CO}$

$.00203 E+C 0$

. $0 \operatorname{tg} 1 \epsilon E+C 0$

$1.040 \mathrm{CLE}+C$

$1.00255 E+C 0$

$1.0,514 E+C$

1. 1 Y 7 E IE +CO

$1.10321 t+50$

1. $106155+60$

1. IUSC SE +CO

$1.112 \angle G E+C O$

$1.115 u$ U + CO

$1.118 \mathrm{c}: 5 t+c u$

1. $1211 \mathrm{bE}+\mathrm{C}$

1. $1213 \mathrm{SCl}+\mathrm{CO}$

l.

1. 193

1.113 se +CO

1. $140 \mathrm{U}, E \dot{E}+\mathrm{CO}$

1. $14464 E+C$

$1.1474 C E+C O$
$1.15 U S 2 E+C O$
$.084345+C U$

. 0 d $4 E 7 E+C L$

$1.085145+C U$

. CAS $7^{15 t+C O}$

$1.08644 t+C 0$

- LS9736E +CU

- Ci甘E3 $2 t+C$

$.005402+C 0$

1. $09 C 64 \mathrm{t}+\mathrm{LU}$

$1.64348 t+60$

$1.09504 t+C$

- $01671 t+C$

. $10033 t+c 0$

. $10229 t+C$

. $10434 \mathrm{~L}+\mathrm{C}$

loeturt co

a.

11339t $+C 0$

$1.11545[+C U$

1. $11838 \mathrm{~L}+\mathrm{Co}$

$1.12059 r+C 0$

$1236 t L+C$

1.1792

1. $1320 \mathrm{CE}+\mathrm{Cu}$

$1.135 \mathrm{Clt}+\mathrm{C}$

1.1 J 1 CCL+CO

$1.141 \mathrm{Cr}+\mathrm{C}$

$1.14413 \mathrm{t}+\mathrm{Co}$

1. $14727 L+C 0$

$1.13 C 40 E+C U$
$1.15370 t+01)$ $\begin{array}{ll}1.11252 E+00 & 1.14745 E+00 \\ 1.11138 E+00 & 1.14468 E+00\end{array}$

l. $11044 E+0 O$

1.1 Cusbt +00

$1.10910 E+00$

1. 1087 UEt0

1. $16947 t+00$

$1.10840 E+U 0$

$1.10850 E+00$

. $10870 E+00$

1. 1 C917E+00

$1.10972 t+00$

l. $110425+00$

$1.11126 t+00$

- $11335 E+00$

. $11456 E+00$

$1.115+4 E+00$

1. $11741 E+00$

$11900 E+00$ 12071:+00 2525+00

$.12443 \mathrm{E}+00$

$1.12644 \dot{1}+00$

1. 12 月5SE+UO

$1.13076 t+00$

1. $133 u^{5} 5+00$

$1+135+3 t+0$

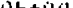

$14570 t+00$

. $151305+00$

1. $154<0 t+00$

1. $15742 \mathrm{t}+00$
$1.14468 E+00$

1. 13 H $91[+00$

$1.13758 t+00$

$1.21068 E+00$

1. $13450 E+00$

$1.13314 E+U 0$

$1.13200 t+00$

$1.13106 \mathrm{E}+00$

$1.13032 \mathrm{E}+00$

$1.12478 E+00$

$1.12742 t+00$

$1.12425 t+00$

1. $12426 L+00$

. $12978 \mathrm{~F}+00$

$1.13028 \mathrm{E}+00$

- 13 lat +Do - $13272 \mathrm{t}+00$ $.133825+00$

131306 E+00

$1.13 \pi 43 E+00$

$1.137 y 3 k+00$

l. $14314 t+00$

1.14 14 L

$1.14118 \mathrm{E}+00$ 14435E+00 1 1625000 ins $58 t+00$ 1. 1 s

1.15644t+vo

1. 1 o $160 \mathrm{~L}+00$
$1.14971 F+C 0$

1. $18500 E+C 0$

1. $18060 E+C 0$

1. $176326+00$

1. $1 / 274 t+00$

1. $13926 t+00$

$1.160 C 6 F+C O$

$1.1611 \mathrm{bE}+\mathrm{CO}$

1. $16050 \mathrm{O}+\mathrm{CO}$

1. 1 ' 5 \& 1 $2 t+00$

1. 1\$600E + 00

1. $1.1413 E+C O$

l. $13110 t+00$

$1.149948+C O$

1. 14894E +CO

1. $4 B 2 B E+C D$

1. $14773 E+C O$

- la 74 lf tCO

政

.14 735t+co

$1.14801 E+C 0$

1. $14 a 60 \mathrm{E}+\mathrm{CO}$

$1.149360+01$

1. $150=78+C 0$

$1.15134 f+00$

$1.15253[+00$

1 1h $3911+00$

$1.1524 C E+C D$

1. $15703 r+00$

$15075^{5}+C 0$

1. loutb tou

1.

1. lotetitoco
$.24011 E+O D$

$1.23304 t+0 u$

1.22646E+OU

- $22023 t+00$

$.2143 \mathrm{HF}+00$

$.208828+120$

1. $20378 E+U U$

$1.1 Y 90 \mathrm{LE}+00$

1. $14458 E+00$

$100405+00$

- $19040 \mathrm{E}+00$

10

$.18327 \mathrm{E}+00$

- $180125+00$

- $17727 e+00$

. 174 CLtOU

. $17242.5+00$

1704CE+UU

$8 t+00$

$.165911+00$

作

$16411 E+0$

- $1035 e t+U 0$

$1.16321 t+00$

$1.14308 t+00$

$1.16315 E+00$

1. $10341 \mathrm{~L}+0 \mathrm{O}$

$10163865+0$

1. 1044 ti

$261+110$

作

作

$1.170115+0$

1. $71040+00$

$1.113416+00$

$1.25943 E+0 D$

$.2897 U E+00$ $.28047 E+00$ $1.27172 E+00$ $.26344 E+00$ $1.25562 \mathrm{E}+00$

$-24 \mathrm{~A} 25 E+00$

$1.24131 \mathrm{E}+0 \mathrm{O}$

. $13400+00$

- $2400 E+0$

- $22300 E+00$

- 21 TRYE+0O

1. $2 C B 14 E+0 U$

. $2 C$ I $9 \mathrm{BE}+0 \mathrm{OU}$

- $2 \mathrm{COL} 3 \mathrm{E}+00$

. $15341 E+00$

- $15 C h 3 E+00$

$O E+U D$

1 Q5U日E+00

I $1 E 369 E+U 0$

1. 1 E1 $18 E+00$

1. $18034 E+00$

$1.17935 E+00$

$117773 t+44$

$1.1772 \mathrm{OE}+U 0$

1.17705E+00

$1.17703 \mathrm{E}+00$

1.17703e+00

i. 17765 E +0

$1.17822 E+U C$

1. 
GAHMA $=1+(1-n)((2 S R /(1-n))(R H C G / H H C)-1)(1-n) R H C / H+C E) \$ 5+W(2 S W-2)$

$$
S H=1.30 \quad R H C G=2.10 \quad S h i=1.20
$$

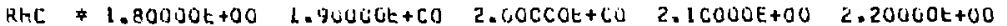
(1)

$1.00000 t-02 \div 1.360436+00$ $2.00000 E-02 \Rightarrow 1.35558 t+00$ $3.000001 .02+1.343] 4[+00$ $4.00000 E-0 ?+1.33168 E+00$ $5.000001 .02+1.32000 E+00$ $6.000001-02 * 1.31007 t+00$

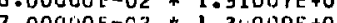
$8.00000 \mathrm{t}-02 * 1.29064 \mathrm{t}+00$ $9.00000 \mathrm{r}-02 * 1.28171 \mathrm{E}+00$ $1.00000 \mathrm{t}-01 \neq 1.27328 \mathrm{E}+00$ $1.10000 F-01 * 1.26334 E+00$ $1.20000+-01 * 1.25708 t+00$ 1. $30000 \mathrm{H}-01 * 1.250 \mathrm{HBE}+00$ $1.40000 E-01 * 1.24433 L+00$ $1.50000[-01 * 1.230211+00$ $1.60000 F-01 * 1.23252 E+00$ $1.70000 L-01 \$ 1.22725 E+00$ $1.80000 t-01+1.22237 t 5+00$ $1.900001-01+1.217 \mathrm{A8E}+00$ $2.000001-01+1.213775+00$ $2.100001-01$ 1. $1002 \mathrm{E}+00$ $2.20000 \mathrm{E}-01$ + 1.2 2.20000 $2.30000 t-01+1.203375+00$ $2.40000 t-01+1.20044 t+00$ $2.50000 \mathrm{t}-01+1.19844 \mathrm{E}+00$ $2.60000 E-01+1.19634 \mathrm{E}+00$ $2.70000 t-01+1.19434 t+00$ $2.80000 E-0 L$ \& $1.19303 E+00$ $2.40000 E-01 * 1.14179 E+00$ $3.00000 \mathrm{E}-01 \neq 1.19082 \mathrm{E}+00$ $3.10000 t-01 * 1.19011 \mathrm{t}+00$ $3.20000 E-01$ 1.18965E+00 $3.30000 E-01 * 1.18943 t+00$ $3.40000 \mathrm{E}-01 * 1.18943 \mathrm{E}+00$ $3.50000 \mathrm{t}-01$ *1.16966t+00 $3.60000 E-01+1.19010 E+00$

$1.447065+\mathrm{CO}$ $1.43146 E+C O$ $1.41377 E+C 0$ $1.40079 F+C 0$ 1. $38644 \mathrm{E}+\mathrm{CO}$ $1.31247 E+C O$ 1. 35 \& $+C$ $1.35491 E+C O$ 1.33 म $8 \mathrm{BE}+\mathrm{CO}$ $1.32414 k+C O$ $1.31427 \mathrm{~K}+\mathrm{CO}$ $1.30433 E+00$ $1.21445 E+C O$ $1.2 d 012 F+C O$ $1.277 t 1 t+C O$ $1.270 \cup 2 E+C O$ $1.26273 E+C O$ $1.25541 E+C O$ $1.24957 E+C O$ $1.2436 b E+C O$ $1.238<4 E+C O$ $1.238<4 E+C O$ $1.23322 \mathrm{E}+\mathrm{CO}$ $1.22801 E+C O$ $1.22441 E+C O$ $1.22059 E+C O$ $1.21714 \mathrm{E}+\mathrm{CO}$ 1. $2 \angle 4 C O E+C O$ $1.21133 E+C O$ 1.20日G3E + CO $1.20893 E+C O$ $1.20510 E+00$ $1.20510 \mathrm{E}+00$ 1. $20344 \mathrm{E}+00$ $1.20247 \mathrm{E}+\mathrm{CO}$ $1.201 \mathrm{DUE}+\mathrm{CO}$ 1. $20096 E+C 0$ 1. $200 O D E+C O$
$1.53245 t+C$ $1.518 C 1 t+C 0$ $1.49 E 43 \mathrm{E}+\mathrm{CO}$ $1.479 t y t+C u$ $1.46176 t+C O$ $1.444 E 3 E+C U$ $1.44463 E+C O$ $1.42 \mathrm{C2 \textrm {HE } + C \mathrm { CL }}$ $1.41269[+C D$
$1.39784 E+C D$ 1. $3837 C L+C O$ $1.37 C 27 t+C u$ $1.35753 \mathrm{t}+\mathrm{CO}$ 1. $34545 \mathrm{E}+\mathrm{CO}$ 1. $\$ 34 C 2 E+C O$ $1.3232 L+C O$ 1. $313(2 t+C)$ $1.30343 \mathrm{E}+\mathrm{CO}$ $1.23441 E+C U$ $1.28545 \mathrm{~L}+\mathrm{Cu}$ $1.278 \mathrm{C} 4 \mathrm{C}+\mathrm{CO}$ $1.27065 t+C u$ $1.26378 E+C O$ $1.2574 C E+C O$ $1.2515 \mathrm{CE}+\mathrm{CO}$ $1.24607 t+C O$ $1.2410 \mathrm{AE}+\mathrm{CU}$ $1.23653 E+C U$ $1.23239 t+C 0$ $1.22866 \mathrm{t}+\mathrm{CU}$ $1.22532 \mathrm{E}+\mathrm{CU}$ $1.22236 \mathrm{E}+\mathrm{Cu}$ $1.2197 \mathrm{BE}+\mathrm{CU}$ $1.21751 t+C 0$ $1.21560 E+C U$ $1.21401 E+C O$ I. $21272 \mathrm{E}+\mathrm{CO}$
$1.04007 E+00$ $1.615 \times 2 E+00$ 1. $59147 \mathrm{E}+00$ 1. $569 U L E+D O$ 1. $54700 E+00$ 1. $52593 E+00$ $1.50578 t+00$ $1.48651 E+00$ $1.46812 \mathrm{E}+00$ $1.450 \mathrm{PE}+0 \mathrm{O}$ $1.43385 E+00$ $1.41794 \mathrm{E}+00$ 1.402 JEtUD 1. $38845 E+0 C$ 1. $37483 E+00$ 1. $36193 E+00$ $1.34473 \mathrm{E}+00$ 1. $33322 \mathrm{E}+00$ 1. $32737 \mathrm{E}+00$ 1. $31716 E+00$ 1. $3075 \theta E+00$ $1.29861 E+50$ 1. $29022 E+00$ 1. $28240 E+00$ $1.27513 E+00$ $1.28839 \mathrm{E}+00$ $1.26217 t+00$ 1. $25645 E+00$ 1. $25121 E+00$ $1.24643 E+00$ 1. $24210 E+00$ 1. $23820 E+00$ $1.23471 E+00$ 1. $231 b 3 E+00$ 1. $22693 \mathrm{E}+00$ 1. $22600 E+00$

\begin{tabular}{|c|c|c|c|}
\hline $\begin{array}{l}34 E+00 \\
30 E+00 \\
30 E+00 \\
3 B E+00 \\
14 E+00 \\
34 E+00 \\
23 E+00 \\
72 E+00 \\
77 E+00 \\
36 E+00 \\
9 E+00 \\
2 E+00 \\
24 E+00\end{array}$ & $\begin{array}{l}1.88 \\
1.84 \\
1.81 \\
1.8 d \\
1.74 \\
1.71 \\
1.69 \\
1.66 \\
1.63 \\
1.81 \\
1.59 \\
1.56 \\
1.53 \\
1.51 \\
1.44 \\
1.47 \\
1.46 \\
1.44 \\
1.42\end{array}$ & $\begin{array}{l}E 3 E+0 U \\
11 E+00 \\
C 5 E+00 \\
42 E+0 C \\
18 E+00 \\
32 E+00 \\
78 E+00 \\
55 E+00 \\
6 C F+00 \\
89 E+00 \\
39 E+00 \\
C 8 E+00 \\
92 E+00 \\
8 Q E+00 \\
54 E+00 \\
07 E+00 \\
24 E+00 \\
41 E+00 \\
56 E+00 \\
67 E+00 \\
70 E+00 \\
62 E+00 \\
42 E+0 C \\
06 E+0 C \\
51 E+00 \\
7 E E+00 \\
77 E+00 \\
52 E+00 \\
38 E+00 \\
13 E+00 \\
35 E+00 \\
41 E+00 \\
66 E+00 \\
15 E+00\end{array}$ & $\begin{array}{l}00 \\
00 \\
00 \\
00 \\
00 \\
00 \\
00 \\
00 \\
00 \\
00 \\
00 \\
00 \\
00 \\
00 \\
00 \\
00 \\
00 \\
00 \\
00 \\
00 \\
00 \\
00 \\
00 \\
00 \\
00 \\
00 \\
00 \\
00\end{array}$ \\
\hline
\end{tabular}

2. 5 COCOE+OO 0 


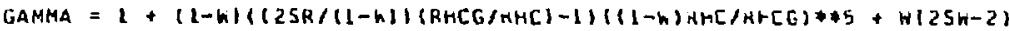

$S H=1.3 C \quad H H C O=2.70 \quad S_{H}=1.20$

$S H=1.3 C \quad H H C O=2.70 \quad S_{H}=1.20$

$S H=1.3 C \quad H H C O=2.70 \quad S_{H}=1.20$

HHC

$2.60000 E+00 \quad 2.700 C L E+C D$

L.00000E-02 $2.35054 E+0 C \quad 2.53565 t+60$

$2.00000 t-02 * 2.24358 E+00 \quad 2.47234 E+C O$

$3.00000 E-0 ?+2.25213 t+002.41174 E+C D$

$4.00000 L-02 * 2.1901 \%$ HOO $2.3532 L E+C O$

$5.00000 L-02: 2.14125 .002 .29614 t+C 0$

$6.0000 u t-0 ?+2.09343:+002.24226 E+C 0$

T.J000OE-02 $=2.0476+1+0 C \quad 2.1848 C E+C O$

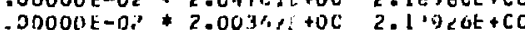

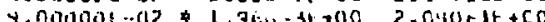

4.

1. 100 .

1.100 1. AR

$1.20000 t-01$ a injul

$1.300001-01 \quad \therefore 90126 t+0 C \quad 1.9142 t E+C C$

$1.40000 E-01 * .77213 i+00 \quad 1.41494 E+C O$

$1.50000 i-01+1.73970 t+0 C \quad 1.83 E 4 E+C O$

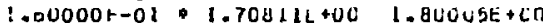

$1.70000 t-01+1.67743 L+O C \quad 1.7 \dot{C} 5 \angle L E+C O$

$1.80000 r-01 * 1.64413 z+00 \quad 1.731$ \%

$1.400001-01 * 1.621$ ett $+0 C$ 1. NUUlet+CO

$2.00000 t-01+1.59343 t+0 C \quad 1.664 t 2 E+C 0$

2.1 UOUNE-01 $* 1.57 L 05[+0 L \quad 1.6464 b t+C 0$

$2.7000 U \mathrm{~L}-01 * 1.54701 \mathrm{t}+00 \quad 2.01347 \mathrm{t}+\mathrm{C} 0$

$2.30000 E-01 * 1.524,7 E+0 C 1.5 C 134 E+C O$

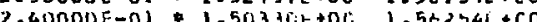

$2.40000-01$ 1.503

2.50010

2.60000r-OR 1.40413L400 1.3L674t+LO

$2.10000 t-01+1.44616 t+001.45560 E+C O$

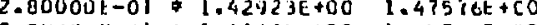

$2.9000 U E-U 1 * 1.4133 U E+O C$ 1.4J7LLE+CO

$3.00000 E-01+1.34 B 34 t+00 \quad 1.43 .33 E+C O$

$3.100001-01 * 1.38431 t 00 \quad 1.42213 E+C 0$

$3.20000 t-01 * 1.37119 t+50 \quad 1.4$ C11 tet +co

$3.300001-01 * 1.33454 L+00 \quad 1.342=7 E+C O$

$3.40000 t-01 * 1.347 B 4 L+0 C \quad 1.37845 E+C O$

$3.50000 t-01 * 1.33096 E+00 \quad 1.36326 E+C O$

$3.60000 t-01+1.32715 t+00 \quad 1.34443 E+C C$






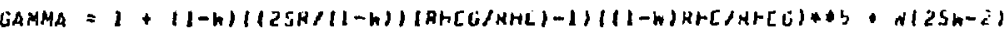

SH $=1.3 \mathrm{C} \quad \mathrm{HHCG}=2.8 \mathrm{C} \quad \mathrm{Sh}=1.20$ (1)

\begin{tabular}{|c|c|c|c|c|c|c|c|c|}
\hline 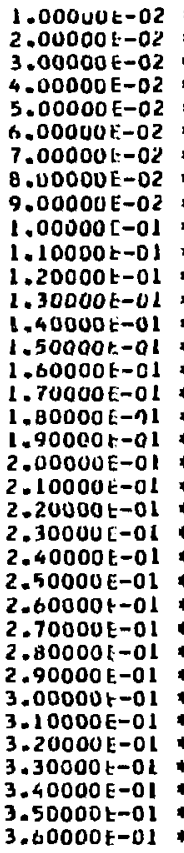 & 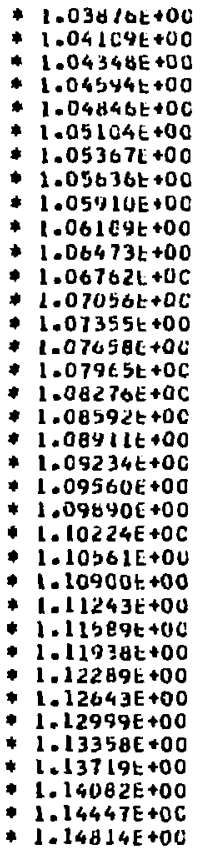 & 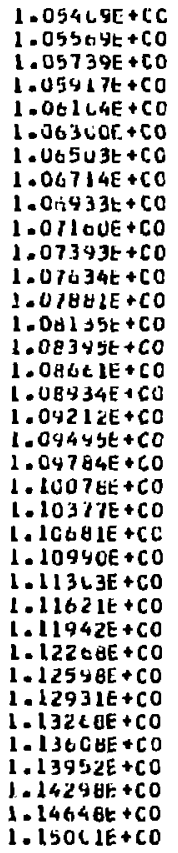 & 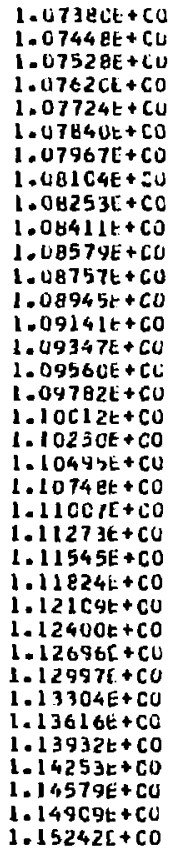 & 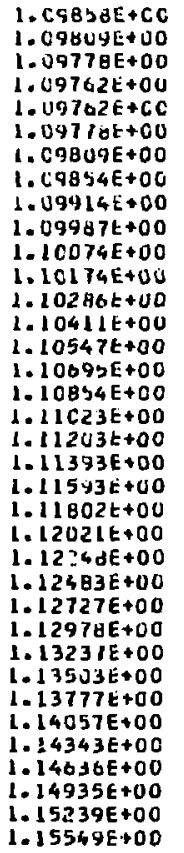 & 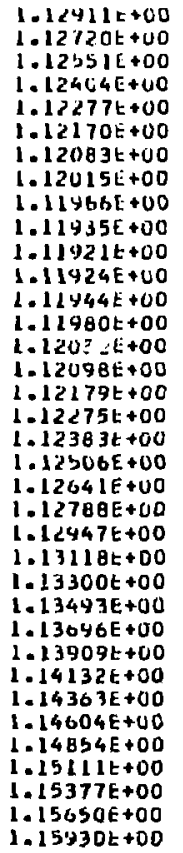 & 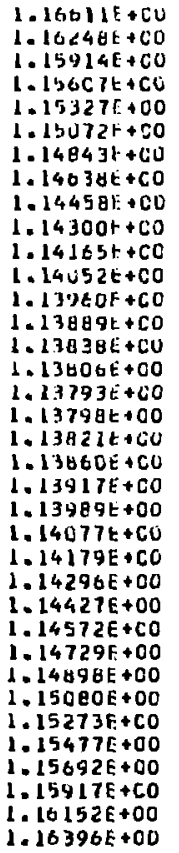 & 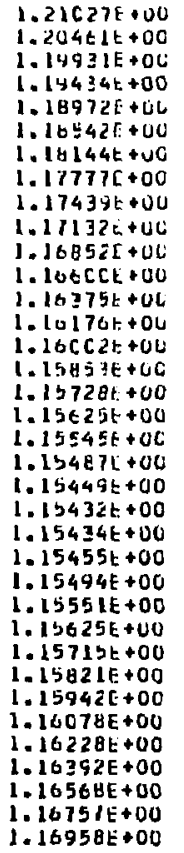 & $\begin{array}{l}1.26231 E+U U \\
1.25427 E+00 \\
1.24667 E+C 6 \\
1.23449 E+00 \\
1.23273 E+00 \\
1.22637 E+00 \\
1.22041 E+00 \\
1.21483 E+00 \\
1.2 C 962 E+00 \\
1.20471 E+00 \\
1.20 C 2 B E+00 \\
1.19611 E+00 \\
1.19232 E+00 \\
1.18892 E+00 \\
1.18544 E+00 \\
1.18277 E+00 \\
1.18014 E+00 \\
1.1770 E+00 \\
1.17589+00 \\
1.17414 E+00 \\
1.17268 E+00 \\
1.17142 E+00 \\
1.17 C 43 E+00 \\
1.16968 E+00 \\
1.16916 E+00 \\
1.16885 E+00 \\
1.16876 E+00 \\
1.16827 E+00 \\
1.16917 E+00 \\
1.16967 E+00 \\
1.17035 E+00 \\
1.17121 E+00 \\
1.17224 E+00 \\
1.17343 E+00 \\
1.17477 E+00 \\
1.17627 E+00\end{array}$ \\
\hline
\end{tabular}


LAMMA $=1+(1-h)(\mid 2 S A /(1-h))($ KHCG/RHL)-L)(1L-h)RHC/HHCG)+DS+W(25H-2)

$S K=1.30 \quad R H C_{U}=2.00 \quad S_{W}=1.20$ $H$

$1.000001-0$ $3.00000-02 * 1.31213 L+00$ $4.00000 \mathrm{E}-02 * 1.24212 \mathrm{E}+0 \mathrm{C}$ $5.00000 t-0.2+1.28289 t+00$ $6.00000 E-02 * 1.27415 E+U C$ $7.600005-02 \cdot 1.265401+00$ $8.00000 L-02+1.21004 t+0 C$ 9.0000Ut-02*1.2507bt+OC $1.000001-01+1.241$ tat +00 $1.00000 t-01 * 1.24340 t+00$
$1.10000 t-01+1.23 \% 40 t+0 C$ $1.10000 t-01 * 1.23740 t+0 c$
$1.20000 t-01 * 1.23135 t+0 c$ $1.20000[-01 * 1.23135 E+00$
$1.30000 t-01+1.225111+00$ $1.30000 L-0 l * 1.22511 t+00$ $1.400005-01 * 1.220472+00$
$1.500001-01 * 1.21962 t 10$ $1.00000 r-01 * 1.21114 t+00$ $1.70000 t=01: 1.201<\angle 200$ $1.80000 t-01+1.203<3 i+00$ $1.40000[-01 * 1.19962 t+00$ $2.00000 L-01+1.19672 t+00$ $2.10000 r-01+1.14+95 c+0$ $2.200001-01+1.19148 t+0 C$ $2.3000 u+01$ 1.109atc 1.16931C+o $2.40000 t-01$ 1.187亿st+0 $2.300001-01$ 1.16303L+0

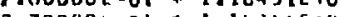
$2.70000 \mathrm{E}-01+1.16344 \mathrm{E}+0 \mathrm{C}$ $2.80000 t-01$ 1.18टt2E+0 $2.90000 \leq-0]+1.16205[+0$ $3.0000 u t-01$ i.14IILL+0 $3.100001-01$ 1.1Elaut+o $3.201000 r-01 * 1.1 \mathrm{EI7It+0}$ 3. 10000r-01 1.162L2t+o $3.40000 t-01+1.18253 L .00$ $3.500001-01+1.10324 k+0 L$

$3.60000 L-01 * 1.18413 t+U C$

1. SCOUGE +C $1.39277 E+C O \quad 1.47 \angle 5 E E+C 6$ $1.37 \forall+B E+C O \quad 1.455 C 7 E+C O$ $1.332 t 6 C+C C$ $1.34 C / 9 E+C O$ $1.32 Y 32 E+C 0$ 1. 3UOC \&E +CC - $29 \mathrm{JCL}+\mathrm{CO}$ 1.2 . l. 2AU Sat + CC $1.2121 \mathrm{CL}+\mathrm{CO}$ 1. $25712 E+C 0$ $1.25 U 94 E+C 0$ 1.244 ULE + CC 1.23 IIL.CO $1.232<4 E+C C$ 1.2275 at + CD $1.222 \%<t+c 0$ 1. 21 a $5 E+C D$ $1.21474 t+C C$ 1. $11+3 E+C J$ $1.2 C H L B E+C O$ $1.26 \cot +\mathrm{CO}$ - $2 \mathrm{Cb}$ cletco

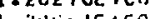
- 2 cusietco $1-1 ; 80<E+C O$ $1.197 L \mathrm{JE}+\mathrm{CO}$ 1.1.b7 $72 t+c$ ? $1.154 a+E+C C$ $1.11]>3[+C 0$ $1.1+34 L E+C O$ $1.14314 k+C D$ I. LUS1Leco $1.1,33 \mathrm{LE}+\mathrm{CO}$

2. $00600 t+c 0$ $2.10000 E+00$

$\angle 20000 E+00$
1. $36292 \mathrm{E}+00$ 1. $54140 \mathrm{t}+00$ I. $52077 E+06$ 1. 50 luzt +00 $1.4 B 211 E+00$ $1.464 C 4 E+00$ 1. $446181+00$ I. $4361810+00$ 1. $43031 E+00$ L. $41461 E+00$ 1. $39 \times U G E+00$ 1. $3634+6 \div 0 D$ $1.371745+00$ I. $3591 J E+00$ 1. $140.85 t+00$ 1. $13 \div 32 E+00$ 1. $32468 E+\angle C$ 1. $31440 E+00$ 1. $36435 t+C$ $1.29502 t+U L$ 1.2873 SDE 00 1. $27945 t+00$ 1. $27207 t+00$ 1. 1 . 1. $26522 E+0$ 1. $\angle 5$ B HOE +O 1. $>5 \angle X+D E+O$ $1.24758 t+00$ 1. $2420 \Omega E+O$ L. $23612 E+00$ 1. $234 \cup 3 E+00$ 1. $3035 E+00$ $1.277005+00$ $1.224156+00$
$1.22100 E+00$ 1.21941E+0C 1. $21755 E+00$ $1.21002 t+00$

\section{$1.66,4425+00$} $1.63843 E+00$ SII - 58956t: +00 $.36002 E+00$ l. $54465 E+U 0$ $1.52362 \mathrm{E}+\mathrm{E} 0$ $1.50351 t+00$ $1.484316+05$ $1.46 j 48[+00$ $1.4402 l i+U C$ $1.49187 t+100$ $1.41604 L+00$ $1.4 O I \cup O E+U O$ I. 30a/3E+00 $1.37121[+40$ $1.36042 t+40$ I. $34 d 33 t+00$ $1.330 y 3 \mathrm{f}+00$ $1.32+20 E+0 C$ $1.3151 \mathrm{lt}+00$ 1. 3ut66t + ic - $30066 \mathrm{t}+\mathrm{UC}$ - 24 rad: +00 $1.21454 \mathrm{c}+00$ $1.28185 t+00$ $1.274711+0 C$ l. 2 की $10 L+00$ $1.26201 \mathrm{t}+00$ $1.25042 t+00$ $1.25131[+0 \mathrm{C}$ 1. 24 BमGE +UD $1.24247 r+00$
$1.23670 t+00$ $1.21354 t+00$ $1.23339 E+U L$ 1. $22902 t+0 a$

\begin{tabular}{|c|c|c|}
\hline 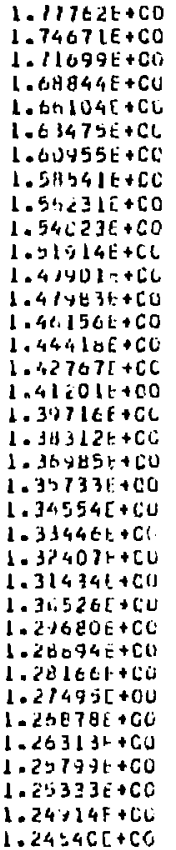 & 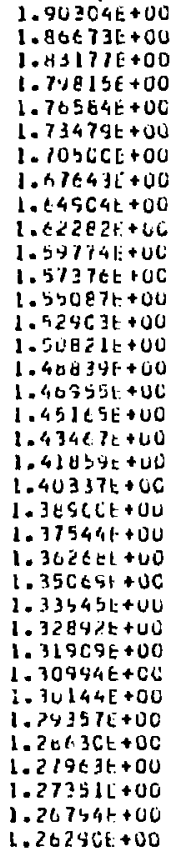 & 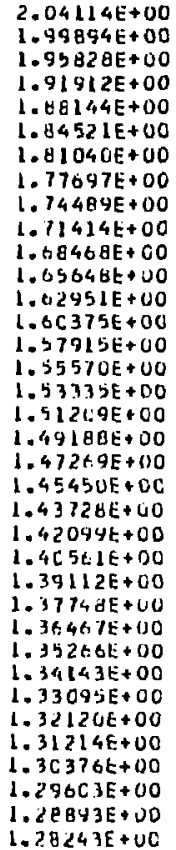 \\
\hline
\end{tabular}

$2.50000 E+00$ $L c+C u$ $1.20349 L+C \dot{U}$ 


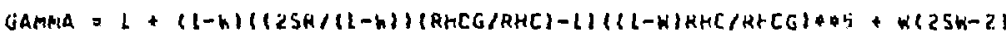
$S K=1.30$
RHCG $=2.80$
$S H=1.20$

RHC $\$ 2.600005+00 \quad 2.700 \mathrm{COF}+60 \quad 2.800 \mathrm{COL}+\mathrm{CG}$

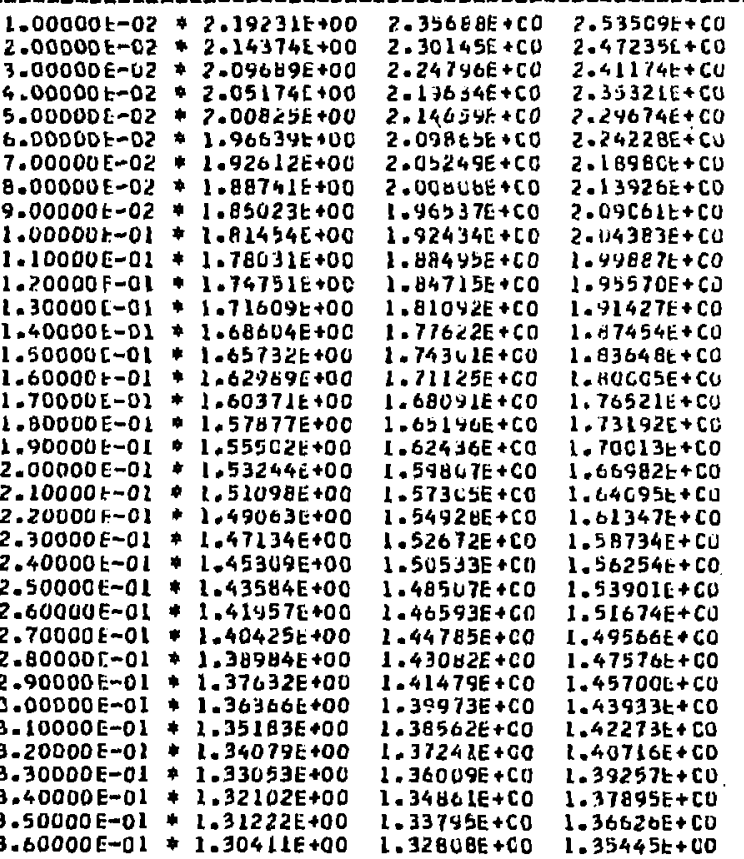




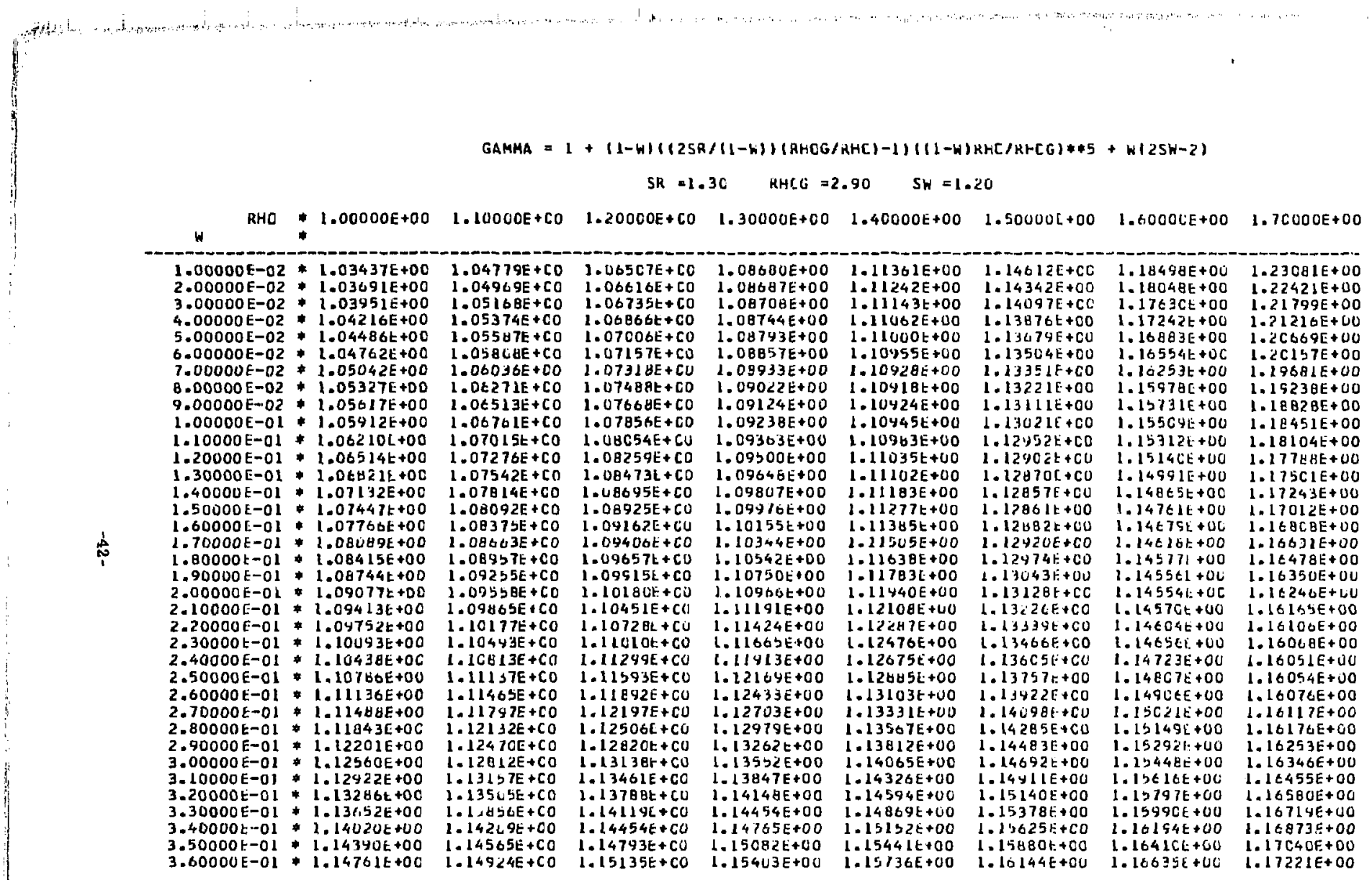


GAMMA $=1+(1-W)(12 S R /(1-h))(R+C G / R H()-1)((I-W) R+C / R+C G)=A S_{1}+W(2 S h-2)$

$S K=1.30 \quad \mathrm{HHCL}=2.4 \mathrm{O} \quad 5 \mathrm{~h}=6.7$

RHo * 1

$1.00000 E-02 * 1.28425 E+00$ $2.00000 E-02 * 1.27521 E+00$ $3.00000 E-02 * 1.26665 E+00$ $4.00000 E-02 * 1.25854 E+00$ $5.00000 E-02 * 1.25088 \mathrm{E}+00$ $6.00000 E-02 * 1.24366 t+00$ $7.00000 E-02 * 1.23686 E+00$ $8.00000 \mathrm{E}-02 * 1.23048 \mathrm{E}+00$ $900000 \mathrm{E}-02+1.22450 \mathrm{E}+00$ 1.00000 E-OL laslog $1.00000 E-01$ 1.21891E+0O $1.10000 E-O 1$ * 1.2137OE+00 $1.20000 E-01$ 1.20887E+00 $1.30000 E \rightarrow 01$ - $1.20439 E+00$ $1.40000 E-01 * 1.20027 t+00$ $1.50000 E-01 * 1.19648 E+00$ $1.60000 \mathrm{t}-01+1.19302 \mathrm{E}+00$ $1.70000 E-01$ 1.18989E+00 $1.80000 \mathrm{E}-01$ - $1.18706 \mathrm{E}+00$ $1.90000 E-01 \div 1.18454 E+00$ $2.00000 E-01 * 1.18230 E+00$ $2.10000 E-01 * 1.18035 E+00$ $2-20000 \mathrm{E}-01 * 1.17467 E+00$ $2.30000 \mathrm{E}-01 * 1.17725 \mathrm{E}+00$ 2.40000 E- 01 * $1.17009 E+00$ . 50000 E 01 1.1750 ..50000E-OI 1.17518E+00 .60000E-01 $1.17450 E+00$ $2.70000 E-01$. $1.17406 E+00$ $2.80000 \mathrm{E}-01$. $1.17383 \mathrm{E}+00$ $2.90000 E-01+1.17382 E+00$ $3.00000 E-01$ 1.17402E+00 $3.10000 E-01+1.17441 E+00$ $3.20000 E-01 * 1.17500 E+00$ $3.30000 E-01 * 1.17577 E+00$ $3.40000 E-01 * 1.17471 E+00$ $3.50000 E-01 * 1.17783 E+00$ $1.17783 E+00$

1.900UOE+CO 2.0UCOUt + CO $2.10000 E+00$ $2.20000 E+00$ c. $30000 E+00 \quad 2.40000 t+00$ $2.50000 E+00$ $1.34593 E+C O$ $\begin{array}{lll}1.34593 E+C O & 1.41646 E+C C & 1.49642 E+0 O \\ 1.3341 C E+C O & 1.40145 E+C O & 1.47185 E+0 O\end{array}$ 1.3B7LJE+CO $1.46007 E+00$ 1.31212E+CO $\quad 1.37346 E+C O \quad 1.44307 E+O C$ $1.3019 b E+C O$ $1.2923 L E+C O$ $1.28318 E+C 0$ $1.27454 E+C O$ $1.36043 E+C O$ $1.34803 t+C U$ $1.33624 \mathrm{E}+00$ $1.32505 E+C O$ $1.25152 E+C O \quad 1.29490 E+C O$ $1.24476 E+C O \quad 1.28594 t+C O$ $1.23843 E+00$ $1.27750 E+C O$ $1.26957 \mathrm{IE}+\mathrm{CO}$ $\begin{array}{ll}1.227 C 3 E+00 & 1.26213 E+C O \\ 1.22194 E+C 0 & 1.25517 E+C O\end{array}$ $\begin{array}{ll}1.22194 E+C O & 1.25517 E+C O \\ 1.21724 E+00 & 1.24867 t+C O\end{array}$ $1.21291 E+00 \quad 1.24263 E+C 0$ $1.20895 E+C O \quad 1.23702 E+C O$ $1.20533 E+C O \quad 1.23183 E+C O$ $1.20207 E+C O \quad 1.22706 t+C O$ $1.19651 E+C O$ $1.19421 E+C$ $1.192 Z \mathrm{OE}+O \mathrm{O}$ $1.19046 E+00$
$1.18904 E+C O$ 1. $18904 E+C O$ $1.1878 B E+C D$
$1.18697 E+0 O$ $1.18631 E+00$ 1. $18590 E+C O$ 1. $18572 E+C O$ $1.28576 E+00$ $1.18602 E+00$ $1.18648 E+C O$ $1.2226 \mathrm{BE}+\mathrm{CO}$ $1.21869 \mathrm{E}+\mathrm{CO}$ $1.21869+C O$ I. $21181 E+C O$ $1.20890 E+O D$ $1.20632 E+C O$ $1.20407 \mathrm{E}+\mathrm{CO}$ $1.20213 E+00$ $1.20050 E+C$ $1.19915 E+C O$ $1.19809 E+C O$ $1.19730 E+00$ $1.19676 E+C O$ $1.19648 E+C O$ $1.42682 E+U O$ $1.41132 t+00$ 1. $39654 E+00$ 1. $38246 E+00$ $1.38246 E+00$ 1.36406E+00 $1.35634 E+00$ 1. $34426 \mathrm{E}+00$ $1.33282 E+00$ 1. 321 YGE+00 1. $11176 \mathrm{E}+00$ 1. $30212 \mathrm{E}+00$ 1. $29304 E+00$ $1.28451 \mathrm{E}+00$
$\mathrm{i}-27652 \mathrm{E}+00$ 1. $26904 E+00$ $1.26207 E+00$ $1.25558 \mathrm{E}+00$ 1. $24957 \mathrm{E}+00$ $1.24401 E+00$ $1.24401 E+00$ $1.2389 D E+D 0$ $1.23422 \mathrm{E}+00$
$1.22995 \mathrm{E}+00$ $1.22995 \mathrm{E}+00$ $1.22608 \mathrm{E}+00$ $1.22259 \mathrm{E}+00$
$1.21948 \mathrm{E}+00$ $1.21948 \mathrm{E}+00$ $1.21673 E+00$
$1.211433 E+0 D$ 1. $21433 E+0 D$ 1. $21226 E+00$ 1. $21051 E+00$ 1. 20907E+00 $1.58038 i$ UO $1.68886 E+C O$ $.56362 t+00$ $1.54219[+00$ $1.52147 \mathrm{t}+00$ $.50163 \mathrm{~F}+00$ $.44265 E+00$ $1.46452 E+00$ $1.44721 E+00$ $1.430695+00$ $.41496 \mathrm{t}+00$ $1.41496 \mathrm{E}+00$ $1.3999 \mathrm{E}+00$ 1. 38 S 76 Et+0 $1.37226 \mathrm{E}+00$ 1. $35945 \mathrm{E}+00$ $1.34733 E+00$ $1.33587 E+00$ $1.32506 E+00$ 1. $31487 E+U D$ 1. $30530 E+00$ $1.29031 E+00$ 1. $28790 E+00$ $1.28004 E+00$ $1.27272 E+00$ $1.27272 E+00$ $1.26593 t+00$ $1.25963 \mathrm{E}+0$ $1.24380 \mathrm{E}+00$ $1.24362 \mathrm{E}+00$ $1.2391 \mathrm{BE}+\mathrm{U}$ 1. $23517 \mathrm{E}+00$ $1.23157 E+00$ $1.22836 \mathrm{E}+00$ $1.22554 E+00$ $1.65990 F+C O$ $1.63400 E+C O$ 1.6041 bE+00 $1.585335+00$ L. $56249 E+C O$ $.54 C 64 E+C C$ $1.51973 \mathrm{E}+\mathrm{CO}$ $1.44975 E+C D$ $1.40068 F+00$ $1.46249 t+C 0$ $1.44515 E+C O$ $1.42866 E^{\circ}+C O$ $1.4129 \theta E+00$ $1.39810 E+C O$ 1. $3 B 39 B E+C O$ 1. $37062 \mathrm{E}+0 \mathrm{O}$ l. $35799 E+00$ $1.346 C 6 E+C O$ $1.33443 \mathrm{E}+0 \mathrm{O}$ 1. $32426 \mathrm{E}+00$ 1. $31434 E+00$ 1. $305 C 5 E+C O$ $1.05636+00$ $1.28827 t+C 0$ $1.28074 E+00$ $1.27377 E+C 0$ $1.26733 E+00$ $1.26141 E+00$ $1.25598 E+00$ $1=25103 E+00$ 1. $24654 \mathrm{E}+00$ $1.24250 E+00$ $1.23889 E+00$ $1.23569 \mathrm{E}+00$ $1.20708 E+00 \quad 1.22097 E+00$

$1.7983 t t+00$ $1.921335+00$ $1.88423 E+00$ 1. $7 E 113 E+00$ $1.65127 t+00 \quad 1.74940 E+00$ $1.62531 E+00 \quad 1.71894 E+00$ $1.60044 E+00$ 1. $0 E 972 E+00$ $1.576635+00$ 1. $6 \in 171 E+00$ $1.553875+00$ 1.634POE+00 $1.53211 E+00$ 1. $10923 E+00$ 1. $1135 E+00$ L 1 . 1. $1135 E+00$ $1.58469 E+00$ $1.47269 \mathrm{t}+00$ 1.53日89E+00 $1.45474 E+0 C \quad 1.5175 \mathrm{EE}+00$ $1.437 t 8 E+00 \quad 1.4572 \mathrm{BE}+00$ $1.45962 E+00$ $1.39162 E+00 \quad 1.44222 E+00$ $1.377 \mathrm{Eg}=+00 \quad 1.42572 \mathrm{E}+00$ $1.34492 E+00 \quad 1.41011 E+00$ $1.35270 E+O D \quad 1.39537 E+U 0$ $1.34121 E+00 \quad 1.38145 E+00$ $1.33043 E+00 \quad 1.36834 E+00$ $1.32033 E+00 \quad 1.35602 E+00$ 1. $31009 E+00$ L. $34446 E+00$ 1.

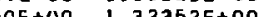
1. $29390 E+00$ I. $32352 E+00$ - 28622E+00 1.3L409E+00 $1.27921 E+00 \quad 1.30533 E+00$ $1.27286 E+00$ 1.29721E+0O $1.26694 E+00 \quad 1.28971 E+00$ $1.26155 E+00$ 1. $28280 E+00$ $1.25665 E+00$ 1. $2764 \mathrm{BE}+00$ $1.19643 F+C O \quad 1.2070 B E+00 \quad 1.21920 E+00 \quad 1.23289 E+00$

$\begin{array}{ll}1.25223 E+00 & 1.27075 E+00 \\ 1.24828 E+00 & 1.26547 E+00\end{array}$ 
GAMMA $=1+(1-h)((2 S R)(1-h))(R H C G / R H C)-1)(11-W) R H C / R H C G)+45+w(2 S W-2)$

$$
S R=1.30 \quad R H C G=2.90 \quad S H=1.20
$$

RHC 2.6000OE+DO 2.700OOE+CO 2.80COOE+CO 2.900OOE+0O

$+$

$1.00000 \mathrm{E}-02+2.05617 \mathrm{E}+00$ $2.00000 E-02 \div 2.01333 E+00$ $3.00000 E-02 * 1.97205 E+00$ $4.00000 \mathrm{E}-02 * 1.93229 \mathrm{E}+00$ $5.00000 t-02 * 1.89404 E+00$ $6.00000 E-02+1.85724 E+00$ $7.00000 t-02 * 1.32189 E+00$ $8.00000[-02 * 1.78793 E+0 C$ $9.00000 t-02 * 1.75534 E+00$ $1.00000 E-01 * 1.72410 E+00$ $1.10000 E-01 \% 1.69416 E+0 C$ $1.20000[-01 * 1.66$ 1.63010t:00 1.3000[-01 1.638jot+00 1.40000 . 50000 -01 - 1.s86soetoo $1.60000 \mathrm{l}-01+1.56305 t+00$ 1.0000 . 1.8000 l1.90000 t-ar *1.49d $13 e+0 c$ 2.00000 E-OL +1.47abiltod $2.10000 E-01 * 1.46009 t+00$ $2.20000 r-01+1.44256 E+0 C$ $2.30000 t-01+1.42597 t+00$ $2.40000 t-01 * 1.41031 E+00$ $2.50000 E-01 \div 1.39344 t+00$ $2.60000 E-01 \div 1.381<4 t+00$ 2.70000r-01 1.36654E+00 2. $80000 \mathrm{~F}-01$ 1.356.34E+00 $2.90000 \mathrm{~F}-0 \mathrm{~L} * 1.344 \mathrm{dBt}+00$ $3.00000 \mathrm{~L}-01+1.33419 \mathrm{E}+00$ $3.10000 \mathrm{E}-01$. $1.32422 \mathrm{E}+00$ $3.200005-01.314475000$ $3.30000 \mathrm{c}-01$ 1.31440+00 $3.40000 \mathrm{E}-01 * 1.29850 \mathrm{E}+00$ $3.50000 E-01 * 1.29123 t+00$ 3.tUUOOR-OL $1.28457 t+00$

$2.20323 E+C O$ $2.15420 E+C$ 2. $10691 E+C$ $2.06132 E+C$ $2.01742 E+C 0$ $1.97515 E+C O$ 1. $93444 E+C O$ 1. $8954 C E+C O$ 1. $45785 E+C O$ 1. $B 2181 E+C O$ $723 E+C O$ $1.754 \angle O E+C O$ $1.69261 \mathrm{ICO}$ $1.66298 E+C O$ $1.63526 E+C 0$ $1.503 \mathrm{CE}+\mathrm{CO}$ $1.55900 E+C O$ $1.53677 \mathrm{E}+\mathrm{CU}$ $1.515 C \mathrm{BE}+\mathrm{CO}$ $1.494 \mathrm{BCE}+\mathrm{CO}$ $1.47499 E+C O$ $1.45653 E+C O$ $1.43909 \mathrm{E}+\mathrm{CO}$ $1.42203 E+C O$ $1.40713 E+C O$ $1.34255 E+C O$ 1. $37886 E+C O$ 1. $36604 E+C O$ 1. $3426 \mathrm{BE}+\mathrm{CO}$ . $3324 \mathrm{BE}+\mathrm{CO}$ $1.3324 \mathrm{EE}+\mathrm{CO}$ $1.313515+C O$ l. $30569 \mathrm{E}+\mathrm{CO}$
$2.36280 t+C 0 \quad 2.53509 E+00$ 2. 30713E+CO $2.47235 E+00$ $2.25339 \mathrm{E}+\mathrm{CC} 2.41174 \mathrm{E}+00$ $2.20155 E+C O 2.35321 E+00$ $2.15157 E+C 0 \quad 2.29014 E+00$ $2.10341 t+C U \quad 2.24228 E+00$ $2.65704 E+C O \quad 2.184 B O E+C O$ $2.01242 t+C 0 \quad 2.13926 E+00$ $1.96952 \mathrm{E}+\mathrm{CO} \quad 2.09061 \mathrm{E}+00$ $1.92830 E+C O \quad 2.04303 E+00$ $1.88872 t+C 0 \quad 1.49881 E+00$ $1.85074 E+C O \quad+.95570 E+00$ $1.81434 E+C O \quad 1.41427 E+00$ $1.81434 E+C O \quad 1.31427 E+00$ $1.7461 U E+C O$ L. $1.71414 t+C O$ I. $00005 E+00$ $1.06370 \mathrm{E}+\mathrm{CO}$ I. $70521 \mathrm{E}+00$ $1.65460 \mathrm{E}+\mathrm{CU}$ L $1.6540 E+C Q$ L. $1.73192 E+00$ l. $00044 E+C D$ L. TOD 1.60044E+CO 1. 66982E+00 1. $55240+C O$ L. $64095 k+00$ $1.5140 E+C O \quad 1.61347 t+00$ 1. $52872 E+C O$ 1. $5 A 734 E+00$ $1.50721 \mathrm{~F}+\mathrm{CO} 1.36254 \mathrm{E}+00$ $1.48685 L+C O$ 1.53401E+0O $1.467 t 0 t+C U 1.516 / 4 t+00$ $1.44943 \mathrm{~F}+\mathrm{CO} \quad 1.49566 E+O C$ $1.43230 \mathrm{~L}+\mathrm{CO} \quad \mathrm{I} .47576 \mathrm{E}+00$ $1.41618[+C U$ 1.457UOE+OO $1.401144 t+C O \quad 1.43933 E+00$ $1.34684 t+C O \quad 1.42273 E+00$ $1.3 / 356 E+C O \quad 1.40716 E+00$ $1.36116 t+C O \quad 1.392575+00$ $1.34961 E+C O$ I. $37845 E+00$ $1.33884 t+C O \quad 1.36626 E+00$ $1.32895 \mathrm{~L}+\mathrm{CU} \quad \mathrm{L} .35445 \mathrm{E}+00$ 
GAMMA $=1+(1-W)((25 R /(1-h))(R+C G / R H C)-1)(11-W) R H(R / R+C G) \neq \$ 5+W(2 S W-2)$

$S K=1.30 \quad H H C G=3.00 \quad S W=1.20$

H

RHC 1.00UCUE+00 \$.10000E+CD 1.20000E+CO 1.3000UE+00 1.40000E+00 1.50600E+00 1.6CCCCE+00 1.7COOOE+00

\begin{tabular}{|c|c|c|c|c|c|c|c|c|}
\hline $\begin{array}{l}00000 E-02 \\
00000 \mathrm{E}-02 \\
00000 \mathrm{E}-02 \\
00000 \mathrm{E}-02 \\
00000 \mathrm{~F}-02 \\
00000 \mathrm{E}-02 \\
00000 \mathrm{E}-02 \\
00000 \mathrm{E}-02 \\
00000 \mathrm{E}-02 \\
00000 \mathrm{E}-01 \\
10000 \mathrm{E}-01 \\
20000 \mathrm{E}-01 \\
30000 \mathrm{E}-01 \\
40000 \mathrm{E}-01 \\
50000 \mathrm{E}-01 \\
60000 \mathrm{E}-01 \\
70000 \mathrm{E}-01 \\
80000 \mathrm{E}-01 \\
90000 \mathrm{E}-01 \\
00000 \mathrm{E}-01 \\
10000 \mathrm{E}-01 \\
20000 \mathrm{E}-01 \\
30000 \mathrm{E}-01 \\
40000 \mathrm{E}-01 \\
50000 \mathrm{E}-01 \\
60000 \mathrm{E}-01 \\
70000 \mathrm{E}-01 \\
80000 \mathrm{E}-01 \\
90000 \mathrm{E}-01 \\
00000 \mathrm{E}-01 \\
10000 \mathrm{E}-01 \\
20000 \mathrm{E}-01 \\
30000 \mathrm{E}-01 \\
40000 \mathrm{E}-01 \\
50000 \mathrm{E}-01 \\
60000 \mathrm{E}-01 \\
\end{array}$ & 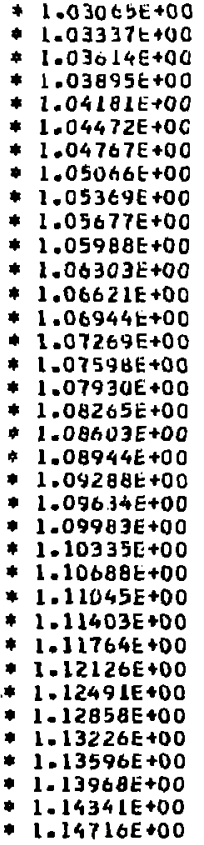 & 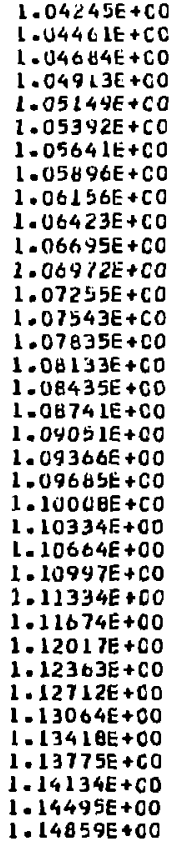 & 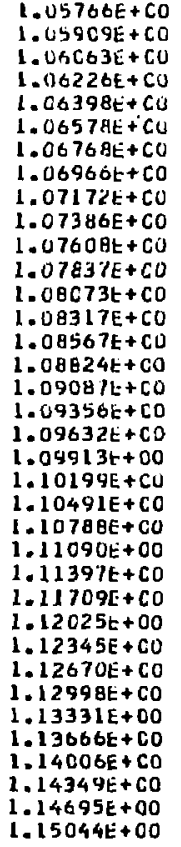 & $\begin{array}{l}1.07680 E+00 \\
1.07733 E+00 \\
1.0780 U E+00 \\
1.07479 E+00 \\
1.07471 E+00 \\
1.08074 E+00 \\
1.08189 E+00 \\
1.08316 E+00 \\
1.0843 E+00 \\
1.08601 E+00 \\
1.08760 E+00 \\
1.08429 E+00 \\
1.09107 E+00 \\
1.09295 E+00 \\
1.09492 E+00 \\
1.0969 E+00 \\
1.09912 E+00 \\
1.10134 E+00 \\
1.10365 E+00 \\
1.10604 E+00 \\
1.10850 E+00 \\
1.11103 E+00 \\
1.11363 E+00 \\
1.11630 E+00 \\
1.11904 E+00 \\
1.12183 E+00 \\
1.12469 E+00 \\
1.12761 E+00 \\
1.13058 E+00 \\
1.13361 E+00 \\
1.23669 E+00 \\
1.13982 E+00 \\
1114300 E+00 \\
1.14622 E+00 \\
1.14948 E+00 \\
1.15279 E+00\end{array}$ & $\begin{array}{l}1.10043 E+00 \\
1.09480 E+00 \\
1.0994 E E+00 \\
1.09922 E+00 \\
1.09415 E+00 \\
1.09923 E+00 \\
1.04947 E+00 \\
1.09485 E+00 \\
1.10038 E+00 \\
1.10105 E+00 \\
1.10186 E+00 \\
1.10280 E+00 \\
1.10386 E+00 \\
1.10505 E+00 \\
1.10657 E+00 \\
1.10779 E+00 \\
1.10434 E+00 \\
1.11099 E+00 \\
1.11274 E+00 \\
1.11460 E+00 \\
1.11356 E+00 \\
1.11662 E+00 \\
1.12676 E+00 \\
1.12300 E+00 \\
1.12532 E+00 \\
1.12773 E+00 \\
1.13021 E+00 \\
1.15278 E+00 \\
1.13541 E+00 \\
1.13812 E+00 \\
1.14090 E+00 \\
1.14374 E+00 \\
1.14665 E+00 \\
1.14961 E+00 \\
1.15264 E+00 \\
1.15572 E+00\end{array}$ & $\begin{array}{l}1.12911 E+00 \\
1.1272 U E+00 \\
1.12551 E+00 \\
1.12404 E+00 \\
1.12277 E+00 \\
1.12170 E+C 0 \\
1.120 E 3 E+00 \\
1.12015 E+00 \\
1.1196 E E+00 \\
1.11935 E+00 \\
1.11921 E+00 \\
1.11424 E+00 \\
1.11944 E+C 0 \\
1.11980 E+00 \\
1.12032 E+00 \\
1.12098 E+00 \\
1.12179 E+C 0 \\
1.12275 E+00 \\
1.1238 E+00 \\
1.12506 E+00 \\
1.12641 E+00 \\
1.12788 E+00 \\
1.12941 E+00 \\
1.13118 E+00 \\
1.13300 E+00 \\
1.13493 E+00 \\
1.13696 E+00 \\
1.13909 E+00 \\
1.14132 E+00 \\
1.14363 E+00 \\
1.14604 E+00 \\
1.14854 E+00 \\
1.15111 E+00 \\
1.15377 E+00 \\
1.15650 E+00 \\
1.15930 E+00\end{array}$ & $\begin{array}{l}1.16343 E+00 \\
1.13993+00 \\
1.1567 C E+00 \\
1.15375 E+00 \\
1.151 C 5 E+00 \\
1.14862 E+00 \\
1.1443 E+00 \\
1.1444 E E+00 \\
1.14277 E+0 C \\
1.14128 E+00 \\
1.14002 E+00 \\
1.13898 E+00 \\
1.13814 E+00 \\
1.13750 E+00 \\
1.1370 E \\
1.1368 E+00 \\
1.13675 E+00 \\
1.13687 E+00 \\
1.1311 E E+00 \\
1.137 E 2 E+00 \\
1.1382 E+0 C \\
1.13902+00 \\
1.1399 E+00 \\
1.14102 E+00 \\
1.14224 E+00 \\
1.14359 E+00 \\
1.14508 E+00 \\
1.146 E 9 E+00 \\
1.14843 E+00 \\
1.15028 E+00 \\
1.15224 E+00 \\
1.15432 E+00 \\
1.15650 E+00 \\
1.15878 E+00 \\
1.16115 E+00 \\
1.16362 E+00\end{array}$ & $\begin{array}{l}1.2 C 394 E+00 \\
1.15 B 37 E+00 \\
1.19355 E+00 \\
1.18896 E+00 \\
1.18449 E+00 \\
1.18044 E+00 \\
1.17670 E+00 \\
1.17327 E+00 \\
1.17012 E+00 \\
1.16725 E+00 \\
1.16467 E+00 \\
1.18235 E+00 \\
1.16029 E+00 \\
1.15848 E+00 \\
1.15692 E+00 \\
1.15559 E+00 \\
1.15450 E+00 \\
1.15363 E+00 \\
1.15298 E+00 \\
1.15253 E+00 \\
1.15229 E+00 \\
1.15224 E+00 \\
1.15239 E+00 \\
1.15272 E+00 \\
1.15322 E+00 \\
1.15390 E+00 \\
1.15473 E+00 \\
1.15573 E+00 \\
1.15689 E+00 \\
1.15818 E+00 \\
1.15962 E+00 \\
1.16120 E+00 \\
1.16291 E+00 \\
1.16474 E+00 \\
1.16670 E+00 \\
1.16877 E+00\end{array}$ \\
\hline
\end{tabular}

$3.60000 \mathrm{E}-01+1.14716 E+00$

$1.14859 E+00$ 1.15044E+00

$1.15279 E+00$

$.15572 E+00$

$1.15930 \mathrm{E}+00$ 
GAMMA $=1+(1-H)(12 S R /(1-W))($ RHOG/RHO)-1)(1)-W)RHC/RHCG)*\$5 +W(2SH-Z)

$S R=1.30 \quad$ RHCG $=3.00 \quad 5 \mathrm{~W}=1.20$

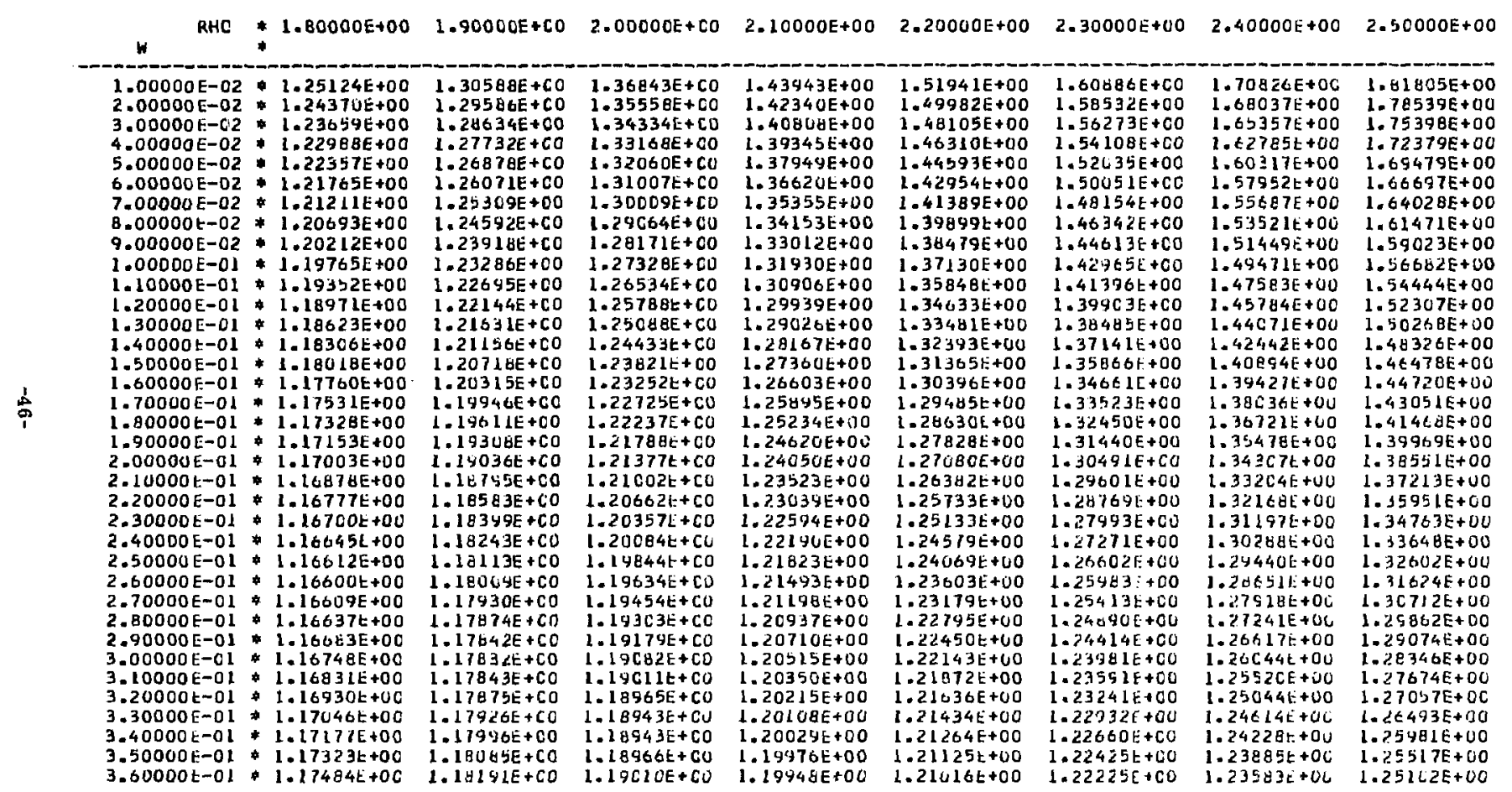




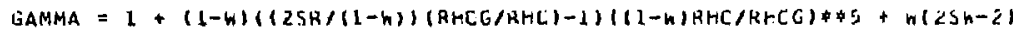

$S R=1.3 \mathrm{C} \quad \mathrm{KHEC}=3.00 \quad S W=1.20$

RHO 2.600OUE+OO 2.7UOLOE+CO 2.80COOE+CO 2.9COOOE+OO 3.COUUOE-30

$1.00000 \mathrm{E}-02+1.930816+00$

$2.07632 E+C O \quad 2.21348 E+C O$ $2.02688 E+C O \quad 2.164011+C O$ $\begin{array}{ll}1.985 U 2 E+C O & 2.1163 L E+C U \\ 1.9447 C E+C O & 2.07032 E+C O\end{array}$

\section{2. $36833 E+00 \quad 2.53509 t+60$} $2.31244 E+00$ 2.47245E+00 $3.00000 E-02 * 1.80434 E+00$ $4.00000 \mathrm{E}-02 * 1 . \mathrm{B2} 2 \mathrm{29E}+00$ $5.00000 E-02 * 1.79559 E+00$ $6.00000 \mathrm{E}-02$ 1.7632 IE+00 $7.00000 E-02 \$ 1.732 \mathrm{LEE}+00$ 9.DODODE-02 *1.70229E+00 $9.00000 \mathrm{E}-02 * 1.67370 \mathrm{E}+00$ $1.00000 k-01 * 1.64631 E+00$ $1.944 \%$ $2.07032 E+C O$ 1. $98331 E+C O$

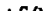
1.0685 C $1.79825=+C D$ $1.44234 E+C O$ $1.90290 \mathrm{t}+\mathrm{CO}$ $1.86501 E+C O$ $.76519 E+00$ 1. $82863 \mathrm{E}+\mathrm{CO}$ 1.73348 E $1.79373 E+C O$ $1.10000 E=01 * 1.62009 E+00$ $1.30000 \mathrm{E}-01 * 1.5710 \mathrm{BE}+00$ $1.40000 E-01 * 1.54623 E+00$ $1.70310 E+C O$ $1.67401 E+C 0$ $1.64618 \mathrm{BE}+00$ $1.61959 E+C O$ $1.76029 E+C O$ $1.72 \mathrm{B26t}+\mathrm{CO}$ $1.09761 E+C O$ $1.06830 \mathrm{r}+\mathrm{CO}$ $1.04031 E+C O$ $1.50000 E-01 * 1.52644 E+00$ 1.56497 $2.25849 E+00 \quad 2.41174 t+00$ 2. $20642 E+00 \quad 2.35321 E+00$ $2.15623 E+00 \quad 2-29674 E+00$ $2.10787 E+D O 2.24228 E+00$ $2.06110 E+00 \quad 2.13980 E+00$ $2.01649 E+00 \quad 2.13+26 E+00$ $1.97340 E+00 \quad 2.0906 I E+00$ $1.93200 E+00 \quad 2.04383 E+00$ $1.89225 \mathrm{E}+00 \quad 1.99887 \mathrm{E}+00$ $1.85411 E+00 \quad 1.95370 E+00$ 1. $81754 E+00 \quad 1.91427 E+00$ $1.78252 t+00 \quad 1.87454 t+00$ $1.748996+00 \quad 1.83648 t+40$ $1.60000 E-01$ * $1.50368 E+00$ 1.546898 $1.54685+C O$ 1.524 पe + 00 1.50402E+CO $1.46536 \mathrm{E}+\mathrm{CO}$ $1.58815 E+C O$ $1.56390 E+C O$ $1.54084 E+C O$ 1.51 $893 \mathrm{E}+\mathrm{CO}$ $1.49813 E+00$ $1.430 t 7 E+C O \quad 1.47843 E+C O \quad 1.53059 E+00 \quad 1.54734 E+00$ $1.74899 E+00 \quad 1.83648 E+00$ 1. 1. $1.652010+00$ I. 70013500 1.62921Etoo $1.10013 E+00$ $1.60266 E+00$ l.66962t+00 $1.57739 E+00$ 1. $1.04095 E+00$ $1.55334 E+00 \quad 1.61347 i+00$ $1.41474 \mathrm{E}+\mathrm{CO} \quad 1.45977 \mathrm{E}+\mathrm{CO} \quad \mathrm{L} \quad \mathrm{5OB} 9 \mathrm{BE}+0 \mathrm{O} \quad 1.56254 \mathrm{E}+00$ $1.39471 E+00 \quad 1.44214 E+C 0 \quad 1.48452 E+00 \quad 1.53901 E+00$ $1.38557 E+00 \quad 1.42550 E+C O \quad 1.46917 E+00 \quad 1.51674 E+00$ $1.37228 E+00 \quad 1.40983 E+C O \quad 1.45091 E+00 \quad 1.49566 E+00$ $1.359 B 1 E+C O \quad 1.3950 B E+C O \quad 1.43369 E+00 \quad 1.47576 E+00$ $1.34 B 13 E+C O \quad 1.38124 E+C U 1.4174 B E+00 \quad 1.45700 E+00$ $1.33723 E+C O \quad 1.36827 E+C O \quad 1.40226 E+0 O \quad 1.43433 E+00$ $1.33723 E+C O$ L. $36825+C 0$ 1.40226E+0O l.43 $33 E+00$ $1.327 C E E+C O \quad 1.35615 E+C O$ 1. $3 A 799 E+00 \quad 1.42273 E+00$ $1.31704 E+C O \quad 1.34483 E+C O \quad 1.37463 E+00 \quad 1.40716 E+00$ $1.30 B 9 E+00$ 1.334.3E+CO 1.36216E+00 1.3925TE+00 $1.30082 E+C O$ 1.32434E+CD $1.35055 E+00$ 1.3TS95E+DO $\begin{array}{llll}1.29340 E+C O & 1.31551 E+C D & 1.33976 E+00 & 1.36626 E+00 \\ 1.29629 E+00 & 1.3071 B E+C O & 1.32977 E+00 & 1.35445 E+00\end{array}$

$\begin{array}{llllll}3.40000 E-O L * 1.27928 E+00 & 1.30082 E+C O & 1.32454 E+C D & 1.35055 E+0 D & 1.37895 E+D O \\ 3.50000 E-01 * 1.27332 E+00 & 1.24340 E+C O & 1.31551 E+C D & 1.33976 E+D O & 1.36626 E+00 \\ 3.60000 E-01 * 1.26790 E+00 & 1.28639 E+00 & 1.30718 E+C O & 1.32977 E+00 & 1.35445 E+00\end{array}$




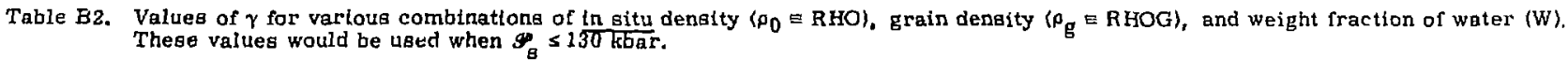

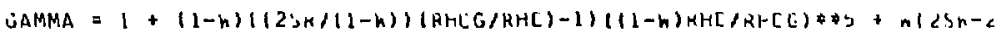

$$
S H=1.3 L \quad K H C L=2.20 \quad S h=1.10
$$

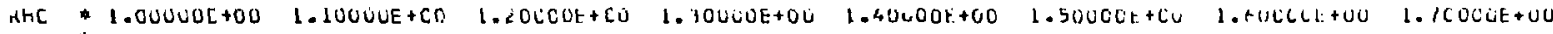
*

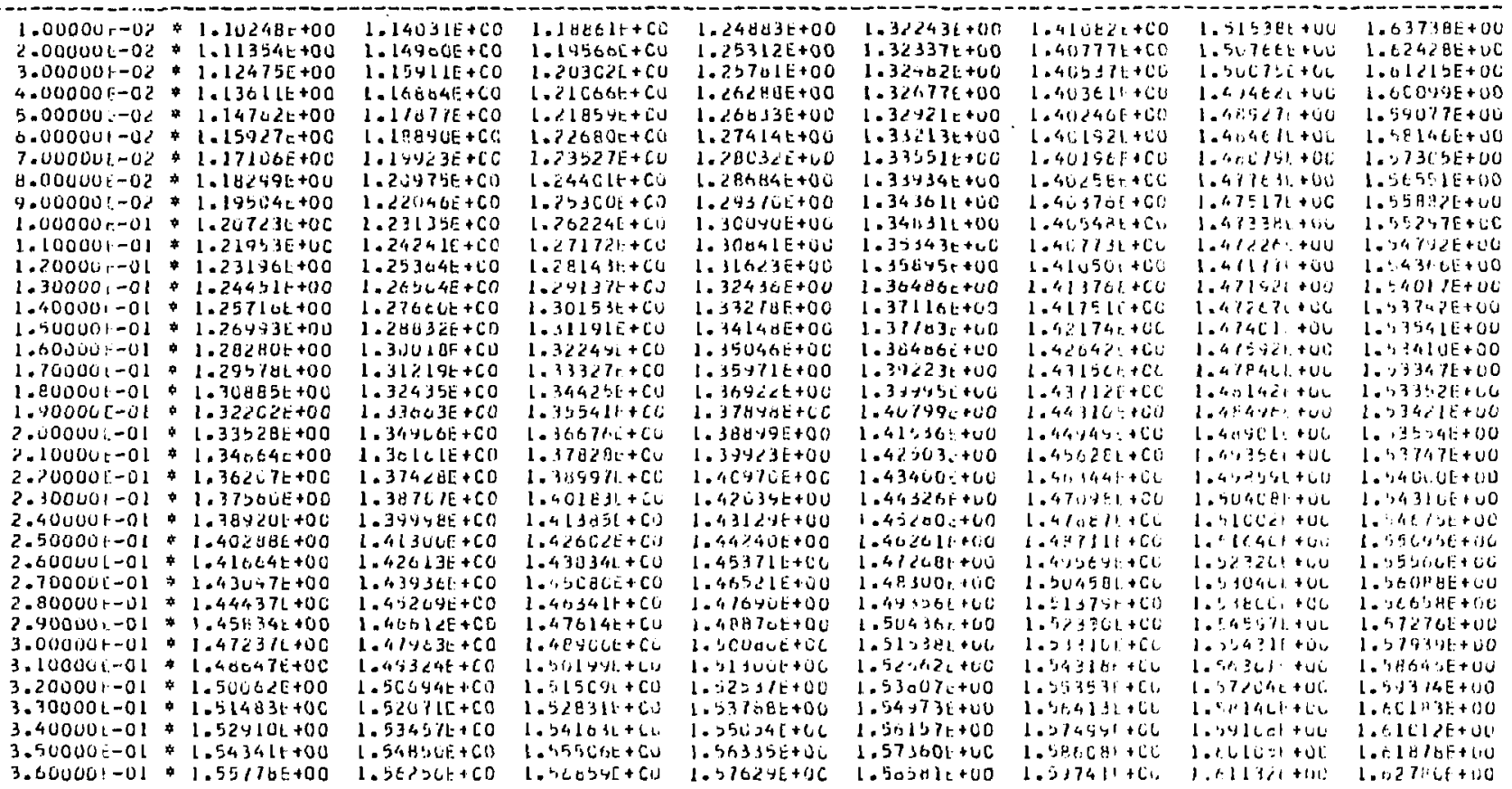


SAIHMA $=1+(1-h)(12 S K)(1-h)(R H[G) K H()-1)(1)-h) R H[/ H+[G)+E+H(2 S H-2)$

$$
S_{\mathrm{N}}=1.30 \quad \mathrm{HHCG}=2.20 \quad \mathrm{SH}=1.70
$$

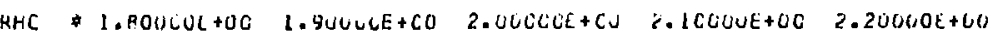

\begin{tabular}{|c|c|c|c|c|c|c|}
\hline 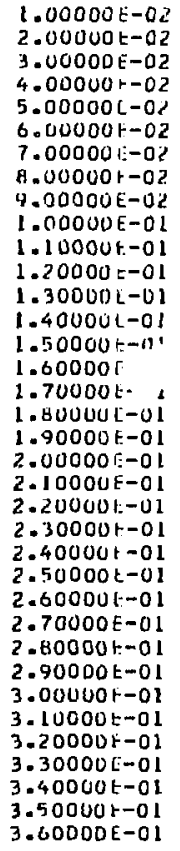 & 4 & 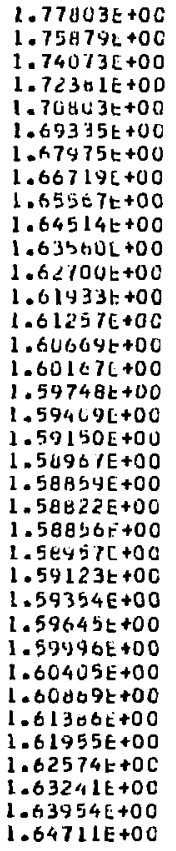 & 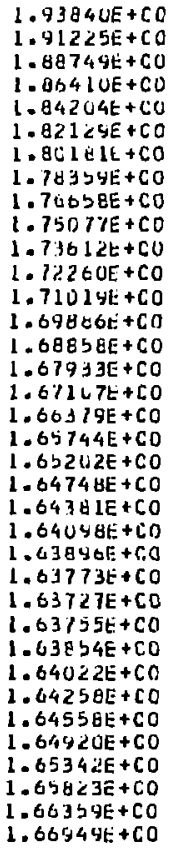 & 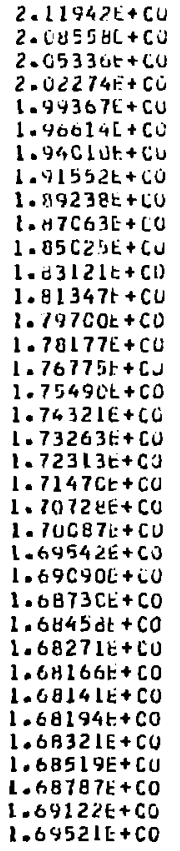 & 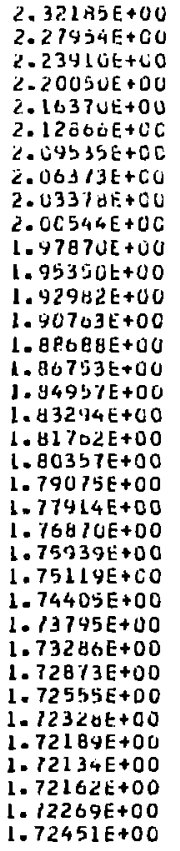 & 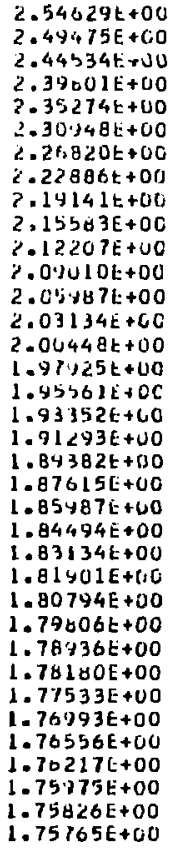 \\
\hline
\end{tabular}




\begin{tabular}{|c|c|c|c|c|c|c|c|c|}
\hline $3 n+35 \times 439=1$ & $3 n+13 i z i, 5-1$ & $x B+3 h \geq z 45=1$ & $0 n+78 x+15 \cdot 1$ & $50+3 \varepsilon 0096=1$ & $5 * 1$ & 31 & & 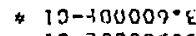 \\
\hline T2+35) & ] & $n 0+7 h 8 n<5 * 1$ & $0 n+3 b R I 05=T$ & $00+35,5 S,-1$ & $0 J+70$ ranc- & $0 J+761$ E $\$ 5=I$ & $20+7 द 1 n \rightarrow 5=1$ & - $10-700005^{\circ} \varepsilon$ \\
\hline$n n+\neg \varepsilon \cup \rightarrow a s=1$ & $n n+28 \geq 31.5 \cdot 1$ & $00+7002 \leq 5=1$ & $00+725075 \cdot 1$ & $00+79 \leftrightarrow 3+5 \cdot 1$ & $n J+\exists l G b E G \cdot I$ & $03+J n c, 6 ? 5 \cdot I$ & $00+38+r_{1} z 5 \cdot 1$ & $\Rightarrow 10-10000 \rightarrow=8$ \\
\hline $0.0+3 n \pi \varepsilon<r=1$ & $0 n+=53645 \cdot 1$ & $00+4 \operatorname{cog} 2 x=1$ & $70+4 b 1085 * 1$ & $00+30 r, 2 \times 4=1$ & $07+-11 \angle 0>5=1$ & $03+\div 4 \geqslant 515 * 1$ & I & $\Xi 0000 E \cdot \varepsilon$ \\
\hline $0.7+15 .+\left\{. c_{1} \cdot 1\right.$ & 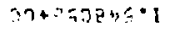 & $00+15738591$ & $x+365525-1$ & $00+\exists F E \rightarrow I C \cdot I$ & 1 & ז' & $30+75,56,6 \cdot 1$ & * IJ-100002. \\
\hline$n n+j e \varsigma b 5 s=1$ & $n n+T I E L E G^{\circ} I$ & $79+\div 75>25 * 1$ & 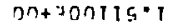 & $00+7 \angle 1105 \cdot 1$ & 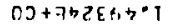 & $05+390.9818 \cdot 1$ & $00+7 n 1784 \cdot 1$ & $* 10-$ \\
\hline $07+31 \cdot: 545 \cdot 1$ & $0 n+1+47 x=1$ & $30+j 75115 \cdot 1$ & $03+? \operatorname{cs} 0 \times 5=1$ & $00+751094=1$ & $00+.78961501$ & $05+\vdots 1 n+21+\bullet$ & $00+76 \Rightarrow 1 \eta \square=1$ & $=10-300000^{\circ} \varepsilon$ \\
\hline$O n+\exists r 9 \forall E<* t$ & $30+: 145975 \cdot 1$ & $23+\therefore \ln 0 n 5^{\circ} i$ & $730+79628 \% 9$ & $00+\exists 1 E S L \% \cdot 1$ & $09+7 z$ 丁4У & $03+71405000$ & $00+\exists E E \leftarrow G \not \bullet 1$ & $* 10-900006^{\circ} 2$ \\
\hline $7 n+704<25 \cdot 1$ & $0 n+4.7735^{\circ} 1$ & $7.2+3 \times ., 4.25=1$ & $30+14 \div 3 ட 401$ & $00+31+20+1$ & $0 J+3 f) 24 h \cdot 1$ & כつナフコタムクタ・T & DO+ $3 T$ TRE & $\Rightarrow 10-700008=2$ \\
\hline $00+7716.75 \cdot 1$ & $72+7 b a \Rightarrow k+=1$ & $30+71 \geq 7 / .5=1$ & $0 า+.75,20 \% \bullet$ & $00+1+x<+2, y=1$ & $07+79 \varepsilon$ s. & 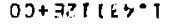 & $30+75 /$ b & $\$ 10-20000 L=2$ \\
\hline $00+301115 \cdot 1$ & $.90 \cdot 125197 \cdot 1$ & $30+3 / 3230^{\circ} 1$ & $57+\pi \operatorname{con}: n=1$ & $03+31>I . E \%=\tau$ & זכ+ & $03+7 h F 11 b * T$ & $5 n+\exists \varepsilon \subseteq n I y-1$ & $\Rightarrow 10-200009^{\circ} 2$ \\
\hline$n n+78 \leq k J 5 \cdot 1$ & 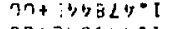 & $0 x+: \angle\left[L \leq \square^{*} T\right.$ & $0 n+32 \cosh 3=1$ & 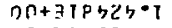 & $20+1.05810 .1$ & 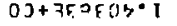 & $20+37 E 76 E \cdot 1$ & $\$ 10-10000 c^{\circ} 2$ \\
\hline $73+745,25 \div \div 1$ & $m+70,501,7 \cdot 1$ & $93+.1013907$ & $2 n+3210 ?+\cdot 1$ & $00+7152[+2 \cdot]$ & $0.2+7 P 6565=1$ & 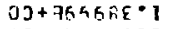 & $70+752736=1$ & \# $10-130004=2$ \\
\hline 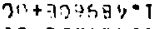 & $30+781130=1$ & $05+711<r+\infty$ & $00+71021 \mathrm{~h}=1$ & $00+31.4035 \cdot 1$ & $n 3+7<3<3 k \cdot 1$ & $0 J+4 b+O L E=1$ & $00+361806=1$ & * IO-J JODOE 2 \\
\hline $07+\exists ?>\operatorname{c⿻}$ & $x+20 e^{2}+2=1$ & 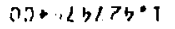 & 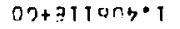 & 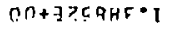 & $n J+7 P 2 b L 5=1$ & $0 J+3 \angle A \geq=1$ & $00+3 n E b 5 E=1$ & $+10-300002 \cdot 2$ \\
\hline $00+7 \zeta>L L+\theta$ & $x n+f ? 25$ ๖タ・ & $n ว+ว 6 ว 81 \bullet \cdot T$ & $0 n+72 b,\{, E=1$ & $70+7 \pi / 0<6.1$ & 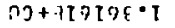 & $0 J+J h C f+b E=1$ & $00+\exists 9 P \cap+E \cdot 1$ & $\$ 10-400001 \cdot 2$ \\
\hline $00+35.21<\div=1$ & $7 n+111<5+1$ & $03+.036 .00 \cdot 1$ & 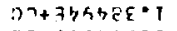 & $77+379498 \cdot 1$ & TEBf=1 & $0.7+35 \geq 855 \cdot 1$ & $92 k \cdot 1$ & $\Rightarrow 10-300000 \cdot 2$ \\
\hline ? & $2 n+1373<+\cdot 1$ & $00+7050 \pi n \cdot 1$ & $0 n+3694 / 8 \cdot 1$ & $\pi 0+\exists \angle O \& 5[\cdot 1$ & $n J+7 \varepsilon \eta \partial \varepsilon \varepsilon=1$ & $E Z E \cdot 1$ & $T E \cdot I$ & $10-100000^{\circ} 1$ \\
\hline $0^{n}+35_{1}: 275=1$ & $2.0+71 \div 5>n \cdot 1$ & $n 0+4681+5=1$ & $20+3 H 7 * 7 \varepsilon=T$ & r.ta $=1$ & $n J+7 c_{j} E$ B?E・I & OD+76SA.DE=1 & $0 n+1+$ & * $10-400008 \cdot 1$ \\
\hline$+3 x+\angle 5+=1$ & $m+45+21+1$ & $B D+i F B F A E=i$ & $07+106+95 \cdot 1$ & 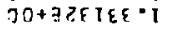 & $0]+-15[2[5 * 1$ & $0 J+7 a \times 0,0,2 \cdot 1$ & $30+7914$ & $\Rightarrow 10-100002 \cdot 1$ \\
\hline $0.9+36,7.55: \cdot 1$ & $7 J+2 \geq \angle[T \%=1$ & כJ+JIJ5/E- & $0 r_{1}+78 F S>E=1$ & $\pi J+7 \geq n \cap 2 F \cdot 1$ & $0.7+1 H 173 E \cdot 1$ & 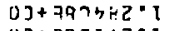 & $30+79<I L 2 \cdot 1$ & * $10-700009^{\circ} \mathrm{I}$ \\
\hline ו & $9 n+11 \geq 77 n-1$ & $07+79727 E=1$ & $n 9+70 \mid<\varepsilon E \bullet 1$ & $00+3>2<08=1$ & I & $0 J+79$ & $00+7$ EOQGZ $\mathrm{I}$ & $* 10-\ni 0000 s^{*} 1$ \\
\hline 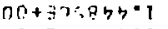 & $37+\div 1104=1$ & $13+1=202 E=\mathrm{T}$ & $0 n+\div 01 / 26 \cdot 1$ & $3 \pi+3 r>6 s ? \cdot 1$ & $03+1+942 z+1$ & $03+365842 \cdot i$ & $n 0+7861, n 2 \cdot 1$ & - $10-t 0000 \rightarrow \cdot \mathrm{I}$ \\
\hline$n n+\exists 15 \% 4 \% \cdot 1$ & 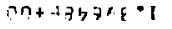 & $00+72585 F \cdot 1$ & $5 n+79 \varepsilon \circ \tau \varepsilon \cdot 1$ & $n 0+3+6.982 \cdot 1$ & $77+\ln$ & $05+ \pm 270 \rightarrow 2 \cdot 1$ & $1 \varepsilon Z \cdot 1$ & $000 E \cdot 1$ \\
\hline$n \because+305 \varsigma+\%=1$ & $77+13 \geq 256=1$ & $T O+A E Z \angle H E \cdot I$ & $0 ก+315: า \varepsilon=1$ & $00+7798 \mathrm{LZ}=\mathrm{T}$ & 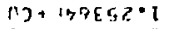 & $0 J+$ HFGEE?・I & $00+7 \geqslant R L 12 \cdot 1$ & $\neq 10-100002-1$ \\
\hline $0 ?+\exists$ டクロク・1 & $B 6 T^{-1}$ & UJ+木לE 1 TE & $n O+7+2 r n \varepsilon \cdot 1$ & $00+310697 \cdot 1$ & $\therefore 5+16 \geqslant+201$ & $00+75^{\prime} 1>2 \cdot 1$ & $00+3$ & I \\
\hline $05+3 ? .150 \%=1$ & $2 n+1075 Q D^{\prime}=1$ & $7 J+-1 R=5, E=1$ & $0 n+1 \angle B F+2=1$ & $00+38 f+5 \geqslant=1$ & $n]+1, E\}$ \&.P・ & $0.5+\exists D \cap R_{1}+2=1$ & $00+3 \cap 41 \mathrm{S1}$ & - $10-700000=1$ \\
\hline 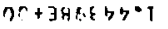 & $20+11529-1$ & $07+1 z \geq \cap \& 6 \cdot 1$ & $00+7 I F 40 ? \cdot 1$ & $30+36 f: 6 h ? \cdot 1$ & 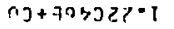 & $0 J \cdot \exists 1.761=1$ & $30+7 \angle 5, Q \angle 1+1$ & * $60-100000-6$ \\
\hline อา+30ทタタタ・1 & 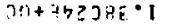 & $27+7 r_{4} ? r_{4} ? E=1$ & $00+\div 90, \angle 2 \cdot 1$ & $00+\exists \mapsto \odot 0 \mapsto 2 * I$ & $03+75 \angle 60 ? \cdot 1$ & $0 J+395805 * 1$ & $30+7 \varepsilon \nabla 671 *$ & $900000 * 8$ \\
\hline $0 n+\exists \measuredangle と ว ク ッ ・ 1$ & $90+4 n \begin{array}{ll}0 \\
0\end{array}$ & $0 D+J 0 \angle n Z E \cdot 1$ & $00+3 b 1>l z \cdot 1$ & $00+7 b 6$. Тह & $17+382561 \cdot 1$ & $0 J+\exists F . T E L 1 \cdot T$ & $00++6125 \mathrm{I}^{\circ}$ & $+20-100000-2$ \\
\hline 0クーヨか・タリが1 & $00+11 \geq 11=1$ & $30+: 125 \Rightarrow 16 \cdot 1$ & $00+7 S s_{1} r_{1} c_{2} \cdot 1$ & $00+\operatorname{Inf} E z Z \cdot T$ & ו & 19 & $00+74704 T \cdot 1$ & $+20-700000 \cdot 9$ \\
\hline $00+39575 \%=1$ & $30+37.4$ & $30+342 ? 15 \cdot 1$ & $00+10 f \alpha \xi 2 \cdot T$ & " & $2.1+1$ & $05+3 \mid 4641 \cdot 1$ & $20+.71$ & $420-700000=\xi$ \\
\hline $20+72 x+45,7=1$ & $0 n+73 n 71.5^{\circ}$ & $0.3+3 E 767 E^{-1} \mathrm{I}$ & $27+704652 \cdot 1$ & $00+308902 \cdot 1$ & $0 כ+7 \rightarrow B B \cap i-1$ & $0 J+3 P C \forall E 1=i$ & $00+3 \angle B+1 T \cdot 1$ & $*<0-300000.4$ \\
\hline $10,57^{\circ} 1$ & $B O 1+\sqcup \mapsto \backsim \rightrightarrows L E=1$ & $75+95770 E=1$ & $00+190 / n ? \cdot 1$ & $00+79+861=1$ & I & $0 J+397121=1$ & $30+\neg$ म & * \\
\hline $0 n+3$ & $00+785 \angle L E=1$ & $\Rightarrow \cap E \cdot T$ & $0 n+כ B D>+P=1$ & $00+\exists T+T S 1=1$ & $03+7096 \rightarrow 1=1$ & $05+73>411 \cdot 1$ & $00+\$ 11050^{\circ}$ & * $20-700000 \cdot 2$ \\
\hline $2 n+39<577 \cdot 1$ & 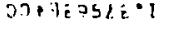 & $20+30<Z 7[\div$ & $0 n+3 T h / F Z \cdot I$ & $07+36 I \mapsto 81 \cdot 1$ & 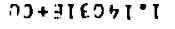 & $0 J+\exists L \geq 5.2 \pi \cdot 1$ & $30+306 \angle 20^{\circ}$ & $20-700500^{\circ} 1$ \\
\hline & & & & & & & & \\
\hline 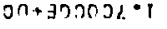 & $1000 \% 0$ & $09+700005=1$ & 100+70000, & $00+3 n 000 \mathrm{~F}=1$ & $n 3+7.250204$ & ו"ום & . 0 & ЈНH \\
\hline
\end{tabular}

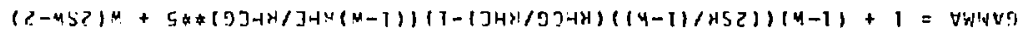


LAMMA $=1+(1-h)(12 S R /(1-h))(R H C G / R H L)-1)((1-h) K H C / H R C E)+\$+W 12 S h-2)$

$$
S R=1.30 \quad R H C G=2.40 \quad S h=1.76
$$

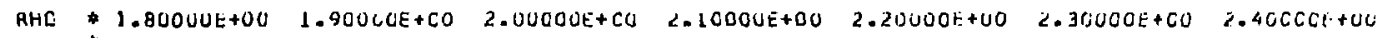

$1.00000 \mathrm{E}-02 \$ 1.57412 \mathrm{E}+00$ $2.00000 \mathrm{E}-02+1.563 \mathrm{HOE}+00$ 3.00000F-02 $-1.55437 \mathrm{E}+0$ $4.00000 E-02 * 1.54582 E+00$ $5.00000 E-02 * 1.5382 L E+00$ $6.00000[-02$ 1. $7.00000 t-02+1.525 ! B t+00$ $8.00000[-02 * 1.51941 E+00$ $9.00000 E-02 * 1.51541 E+00$ $1.00000 t=01+1.51166 t+00$ $1.10000 t-01+1.50864 E+00$ $1.20000 E-01 * 1.50634 E+00$ $1.30000 \mathrm{t}=01 \quad 1.50473 \mathrm{E}+00$ $1.40000 \mathrm{E} O \mathrm{I}$ $1.40000 \mathrm{E}-1.50379 \mathrm{E}+0$ $1.50000 \mathrm{E}-01$ 1.503 1.60000 . $1.70000 \mathrm{R}-0 \mathrm{~L}$ 1.50306E+00 1. 80000E-a1 1. 1.90000 L $1.50062 E+00$ $2.00000 E-01$ * $1.51136 E+0 C$ 2.10000E-OL $1.51465 E+D O$ $2.20000 E-01+1.51847 t+00$ $2.30000 \mathrm{t}-0.1 \neq 1.522 \mathrm{t2E}+00$ $2.40000 \mathrm{E}-01+1.527 \mathrm{~b} 6 \mathrm{E}+00$ $2.50000 E-01 * 1.53299 E+00$ 2.60000E-01 *1.53878E+00 $2.70000 t-01+1.54503 E+00$ $2.80000 E-01+1.55172 t+00$ $2.90000 E-01 * 1.55843 E+00$ $3.00000 E-01$ 1.56635E+00 $3.10000 E-01 * 1.57426 E+00$ $3.20000 E-01 * 1.58255 E+00$ $3.30000 E-02 * 1.59120 E+00$ $3.40000 \mathrm{E}-01$. $1.60021 E+00$ 3.40000E-OL $3.50000 E-01 * 1.60955 E+0 C$
$3.50000 E-02 * 1.61922 E+00$

$1.64305 E+C O$ $1.67809 E+C O$ $.6 E 35 \mathrm{BE}+\mathrm{CO}$ $1.65011 E+C O$ $1.637667=+00$ $1.62614 E+C O$ $1.6157 \mathrm{CE}+\mathrm{CO}$ $1.60615 E+C D$ $1.59752 E+C D$ $1.58979 E+C O$ $1.58294 E+C O$ $1.57695 E+C O$ $1.57178 E+C O$ $1.56743 E+C 0$ $1.56743 \mathrm{~L}+\mathrm{CO}$ 1. $563665+C$ $1.501 C 7 E+C D$ 1.55 $557 t 2 E+C O$ - 57 SE +CO - $55713 E+C O$ . $557 \mathrm{Bat}+\mathrm{C}$ . $55923 E+C$ $1.56123 E+C O$ $1.56382 E+C O$ . $567 L L E+C O$ $1.57076 E+C O$ . 575 C6E + CO I. $579 \forall 9 E+C O$ 1. $58523 E+60$ $1.591 \angle \mathrm{COE}+\mathrm{C}$ $1.59737 \mathrm{E}+\mathrm{CO}$ $1.60414 E+00$ 1. $61130 E+C O$ $1-619 \mathrm{COE}+\mathrm{C}$ $1.62704 E+C O$ $1.63549 E+C D$
$1.82425 t+C 0$ $1.80779 E+C 0$ $1.16854 E+C O$ 1 . $13417 t+c 0$ $1.7317 t+C 0$ 1. $71868 \mathrm{E}+\mathrm{CO}$ 1. $70431 \mathrm{E}+\mathrm{CO}$ $1.691 \mathrm{C} 3 E+C O$ 1. $678 \mathrm{~B} 2 \mathrm{E}+\mathrm{CO}$ $1.66764 t+C 0$ $1.65747 E+C O$ $1.64228 t+C O$ $1.64228 \mathrm{t}+\mathrm{CO}$ $1.63278 t+C 0$ $1.02640 \mathrm{E}+\mathrm{CO}$ $1.62640 E+C O$ $1 . n 2052 E+c 0$ $1.01628 E+C O$ $1.01249 E+C O$ $1.60951 \mathrm{t}+\mathrm{CD}$ $1.60733 E+C O$ $1.60591 \mathrm{E}+\mathrm{CO}$ 1. $60523 \mathrm{E}+\mathrm{CO}$ 1. $60528 E+C O$ $1.40602 E+C O$ $1.60744 E+C 0$ $1.60952 E+C O$ $1.61222 E+C O$ $1.61554 t+C O$ $1.6194 \mathrm{EE}+\mathrm{CU}$ $1.62394 E+C O$ $1.62897 E+C O$ $1.62897 E+C O$ $2.03453 E+C O$ $1.640 E 1 E+C 0$ $1.64717 E+C O$
$1.65422 E+C O$
1. $49168 E+00$ 1. $1.92713 E+00 \quad 2.08292 E+0 Q$ 1. $90200 E+00$ 1. $97026 \mathrm{E}+00$ 1. $45500 E+00$ 1. $834 \mathrm{ABE}+00$ 1. $81504 E+00$ 1. 7966OE +00 1. $17937 \mathrm{E}+00$ 1. $16334 E+00$ 1.734B 1. $12322 \mathrm{~B}+0$ 1. $12220 E+00$ 1. $71080 E+00$ $1.64105 E+00$ $1.68271 E+00$ $1.67535 E+00$ l. $06895 E+00$ $1.6634 \mathrm{dE}+00$ 1. $\$ 5842 E+00$ 1. $65523 \mathrm{E}+00$ $1.65239 E+00$ $1.65038 t+00$ $1.64917 E+00$ $1.64873 E+00$ 1. $34904 E+00$ $1.65007 \mathrm{E}+00$ $1.65181 \mathrm{E}+00$ $1.65422 \mathrm{E}+00$ $1.6572 \theta E+00$ 1. $36097 \mathrm{~T}+00$ $1.66527 E+00$ $1.67015 \mathrm{E}+00$ $1.67580 \mathrm{E}+00$

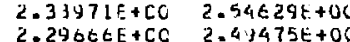
+UC $2.2555 C E+C a$ Za $2.44534 E+0 C$ 2.OSIOLC+OC 2. $210 E C E+C O \quad 2.35 E C 1 E+O O$ $2.02071 E+00 \quad 2.17872 E+C 0 \quad 2.35274 E+00$ $1.9140 t+00 \quad \angle .14302 t+00 \quad 2.30942 t+00$ $1.9647 B E+00 \quad 2.1 C 9 C 7 E+C O \quad 2.2 B B 2 C E+O O$ $1.93901 E+00 \quad 2.07684 E+C 0 \quad 2.2288 E E+00$ $1.71484 E+00$ 2. $04629 E+C 0$ 2.14141E+00 $1.89204 E+00 \quad 2.01737 E+C O \quad 2.155 E 3 E+00$ 1.E $7065 E+C O \quad 1.54 C C 7 E+C 0 \quad 2.122 C 7 L+00$ $1.830625+00 \quad 1.95433 E+C O \quad 2.0401 C 1+00$ $1.83193 E+00$ 1.44U13E+CO $2.45981 E+00$ $1.81455 E+00.1 .94013 E+C 0 \quad 2.03987 E+00$ $1.81455 E+00$ 1.91743E+CO 2.03134E+U0 $1.7944 E+00$ 1.04C19E+CC 2.CC448E+0 $1.7935 \mathrm{C}+00$ 1. $1.75743 E+00$ 1.8579EE+CO $1.955 E 1 E+00$ $1.7543 E+00$ 1.84090E+CO $1.43352 E+00$

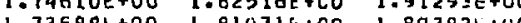

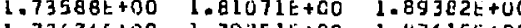
$1.72674 E+00$ 1.79751E+CO $1.47 E 15 E+00$ $1.71866 E+00 \quad 2.78553 E+60 \quad 1.85987 E+00$ $1.71161 E+U D \quad 1.77473 E+C O \quad 1.84494 E+O O$ $1.70534 E+00 \quad 1.76508 E+C 0 \quad 1.83134 E+00$ $1.70045 E+00 \quad 1.75655 E+C 0 \quad 2.619 C 1 E+00$ $1.64628 E+00 \quad 1.74910 E+C O \quad 1.80754 E+01$ $1.69302 E+00 \quad 1.74270 E+C O \quad 1.79 B C 6 E+O D$ $1.69064 E+00 \quad 1.73722 E+C 0 \quad 1.78936 E+00$ $1.64911 \mathrm{E}+00 \quad 1.73292 \mathrm{E}+\mathrm{CO} \quad 1.7 \mathrm{~B} 1 \mathrm{BCF}+00$ $1.68839 E+00 \quad 1.72948 E+C 0 \quad 1.77533 E+00$ $1.68447 E+00 \quad 1.72696 E+C C \quad 1.70593 E+00$ $1.68432 E+00 \quad 1.72533 E+C 0 \quad 1.76558 E+00$ $1.64041 E+00$ 1.725 $1.72456 E+00$ 1.705 $1.64091 E+00 \quad 1.72456 E+00 \quad 1.76217 E+00$ $1.69320 E+00 \quad 1.72463 E+00 \quad 1.75975 E+00$ $1.69984 E+00 \quad 1.72712 E+00 \quad 1.75765 E+00$ 


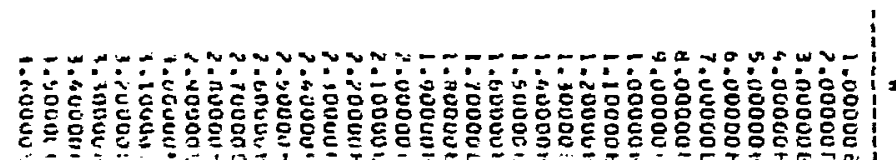

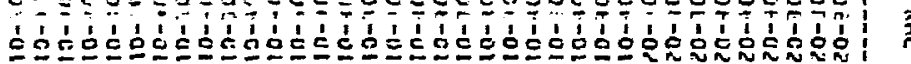

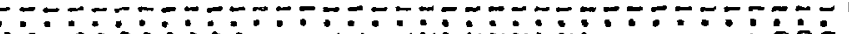

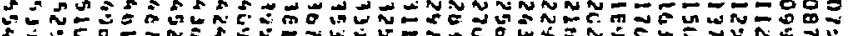

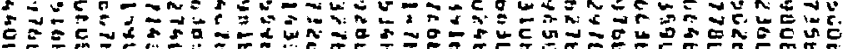

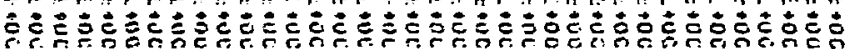

눈

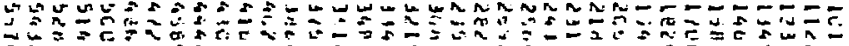

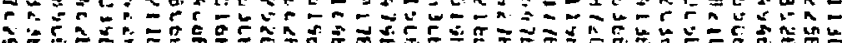

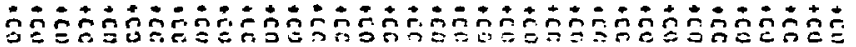

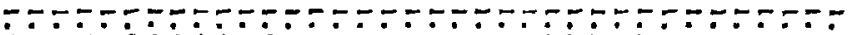

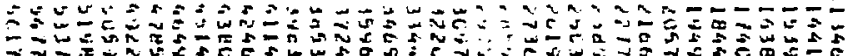

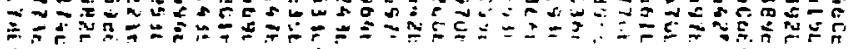
ล้อง

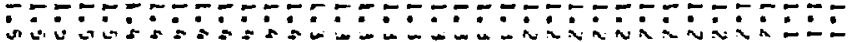

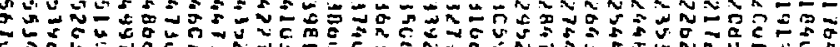

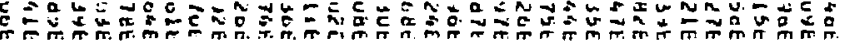

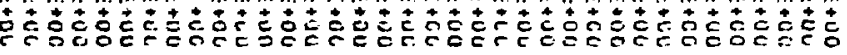

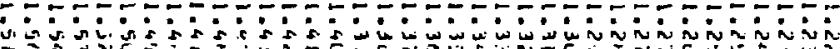

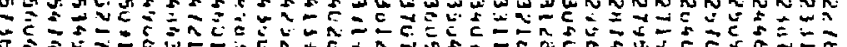

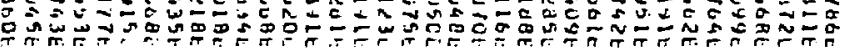

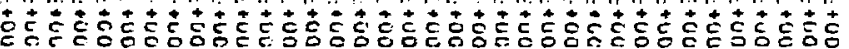

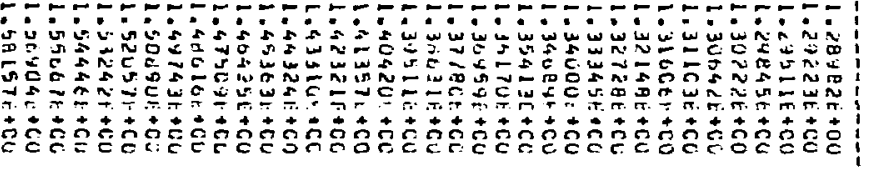

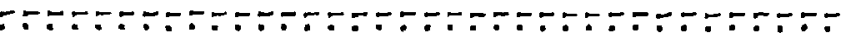

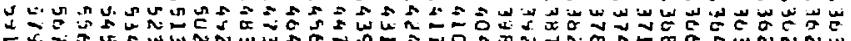

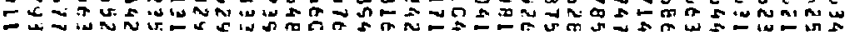

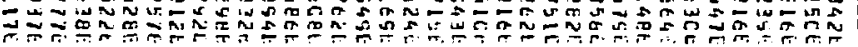

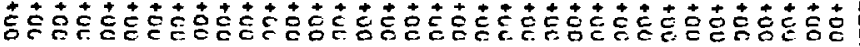

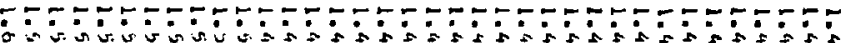

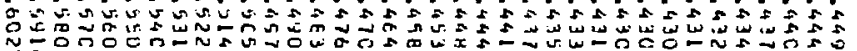

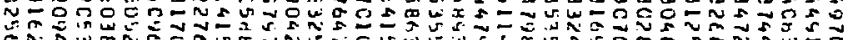

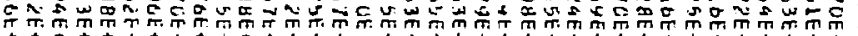

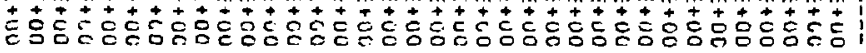




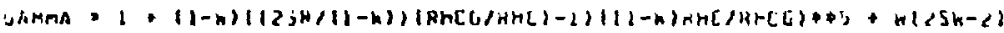

$$
\text { in } \$ 1.30 \quad \text { Anlid }=2.19 \quad 5.4=1.70
$$

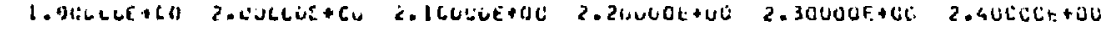

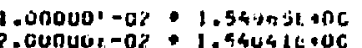

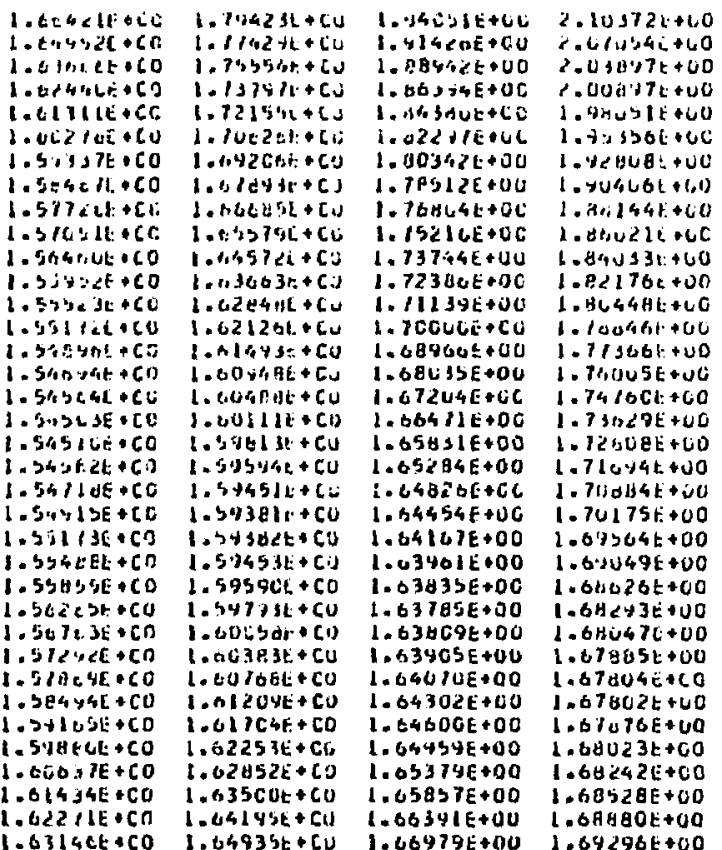

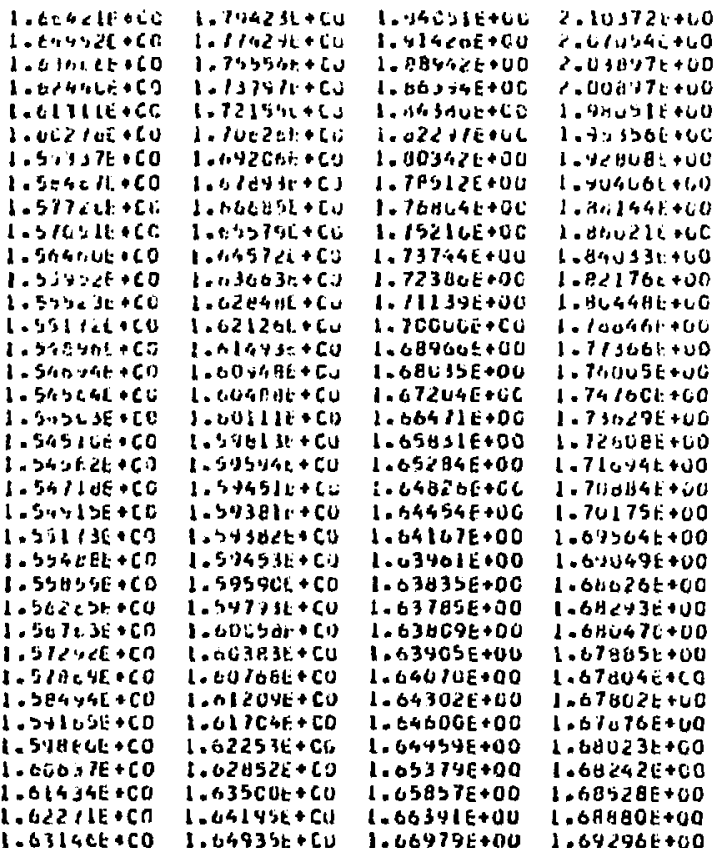

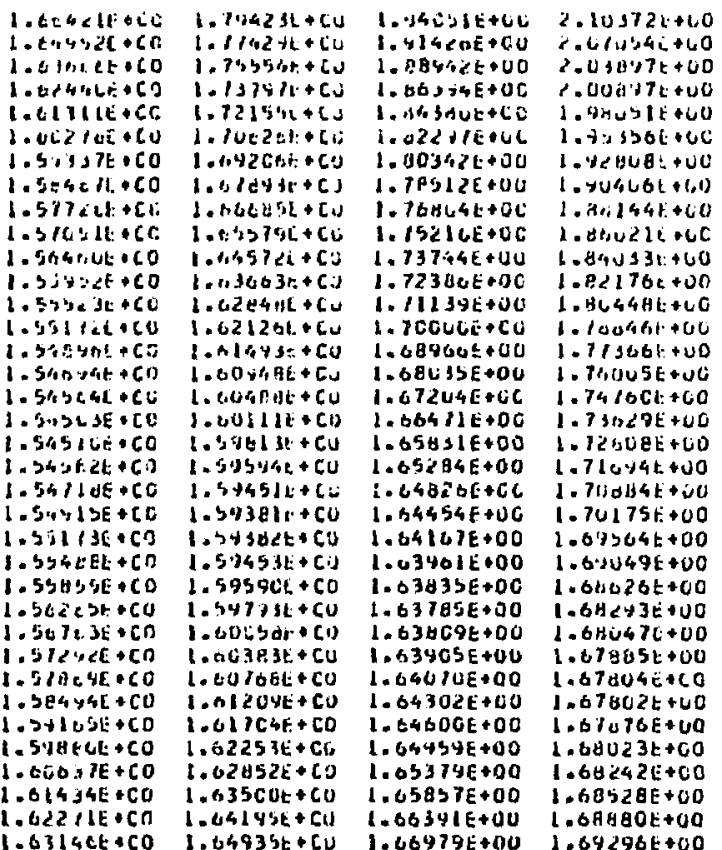
2.cunucr -0 ? : 1.54641L40C $4.000013-02$.

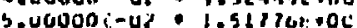

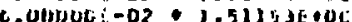

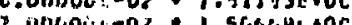

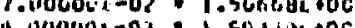

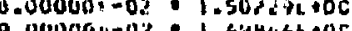

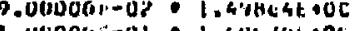

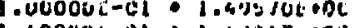
$1.100001-01+1.44 .1471 .100$ $1.20000+-0|:| .48 \mid, 32000$ $1.700001 .01 \cdot 1.4 \times 143200$ $1.400 C u t-01: 1.43 i d i c t+1)$ 1.30000t-Ul 1.4\%1<11.0un

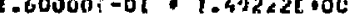
$1.70000 r-01 * 1.4 \% 3+3 L+46$ $1.800061-01: 1.49661600$ $1.00000 L-01 * 1.414751$.0C 2.6110001-01:1.567c4tedac:

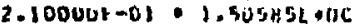
$2.20000 r-01 \cdot 1.961$ ibi+0c $2.101000-01.51540+100$

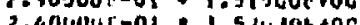
2.

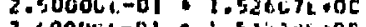
$2.4000 \mathrm{Cl} 0 \mathrm{~d}$ 2.70000 $1.53043 \mathrm{~L}+0 \mathrm{C}$

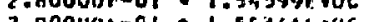
- . 3. 30000 t-01 1.30132k.00

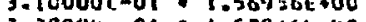
3.2000ur-01 1.57616L 10C $3.30000 \mathrm{~L}-01: 1.58712 \overline{5} \cdot 0 \mathrm{C}$ 3.4000 te-GI 1.54hruito

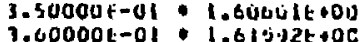
$2.21444 t+C D \quad 2.411313 E+60$ $2.24364 t+60$ 2.43417k+00 $2.2047 E \mathrm{E}+\mathrm{CO} \quad 2.30724 \mathrm{CH}+\mathrm{UO}$ C.167EzF+CO $2.34238 t+U 0$

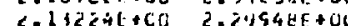
$2.03058 f+i 22.00557+0$

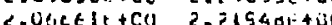

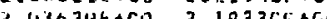

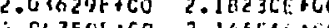
C.C1.7EQt+CO 2.14E5EL+LC 1. $306475+C 0 \quad 2.113411+00$ $1.454901:+C C \quad 2.9 B 162 t+00$ $1.43084 E+C U$ R.

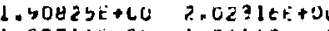
1.8B7IItICC $1.994 .42 t+U 0$ l.oS138t+CI 1.91121E+UO $1.84162 t+C 0 \quad 1.1477 C t+O C$ $1.032 C U F+C D \quad 1.52565 E+00$ $1.81624 E+C U \quad 1.7451 C 1 .+00$ 1.801E4t+CC 1.86tCCLt+00 $1.7 P B 63 E+C H \quad 1.6 .68311+50$ $1.17063 E+C ; ; \quad$ l. E. 3 CCt +60 1. $16580 f+C 0$ l. 1. $155101+C O$ 1. $1.75210++C O \quad 1.0233 \mathrm{BE}+U C$ 1.74 - T2476F+CO I.TSSEzE +UD 1. TJjSOE+CO 1.7858Et+00 - r2aD $k+c 0$ 1. 1.72353e+co 1.77Jare +OO $1.7149 B E+C 0$ 1. $76 t 77 E+00$ $1.71734 F+C C \quad 1.76124 E+00$ 1.71SSSE+CO I.7SE72t +UO $1.71469 E+C O \quad 1.75318 E+00$ $1.1146 .1 E+C 0 \quad 1.75 C E I E+00$ $1.11534+00 \quad 1.74895 C+00$ $1.71683 t+C a \quad 1.74817 t+00$ 1.00000t-01:1.61-j.jet+oc

$1.6314 \mathrm{Ct}+\mathrm{CO}$

$.64935 \mathrm{~F}+\mathrm{CV}$

$1.66979 E+0$

$.64298 E+00 \quad 1.71956 E+C D$

$1.7482 \in E+O C$ 


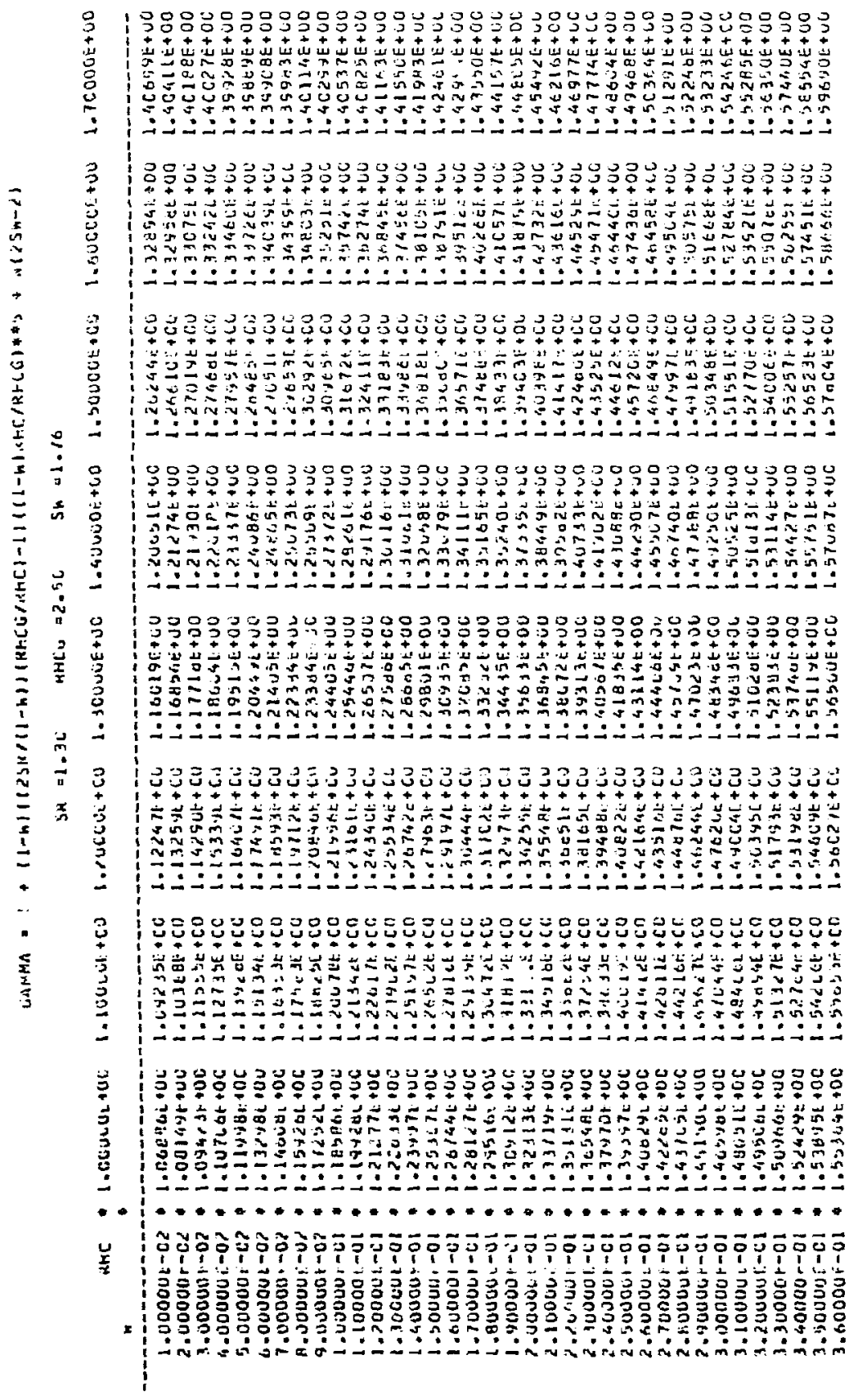




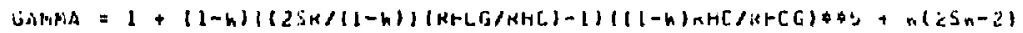

$$
\text { Sk }=1.30 \quad \text { inco }=2.30 \quad 5 n=1.70
$$

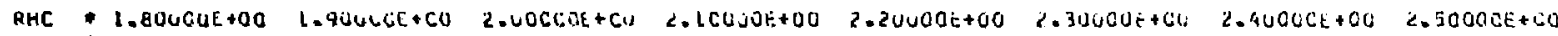

H

I. $0000 \mathrm{E}=0 \%$ 1.49751E+00 $2.00000 E-02+1.440>65+0 C$ $3.00000 E-02 * 1.40414 L+0 C$
$4.00000 E-02 * 1.479 C 6 E+0 C$ $1.00000 E-02 * 1.47966 k+00$
$5.00000 E-0 ? * 1.47442 E+00$ 6.00000t-02 1.470SIE+0C $7.00000 t-02 \quad 1.46130 k+0 c$ $8.00000 E-02+1.464 .79 k+00$ $9.00000 t-02 \div 1.462+25 E+00$ $1.00000 r-01 \quad 1.46176 t+00$ $1.10000 E-C 1+1.46121 t+00$

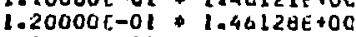
$1.30000 \%-01 * 1.461,5 E+00$ $1.40000 E-01+1.46322 \mathrm{E}+0 \mathrm{C}$ 1.5000 r $1.46303 E+0 C$ $1.60000 \mathrm{r}-01+1.4 a 744 \mathrm{E}+0 \mathrm{C}$ 1 . $1.80000 \mathrm{t}-0 \mathrm{~L}+1.47382 \mathrm{t}+0 \mathrm{C}$ $1.90000 \mathrm{r}-01$ 1.477iaE+OC $2.00000 t-01 * 1.48223 E+00$ $2-10000 t-01+1.48716 E+00$ $2.20000 E-01 * 1.49256 t+00$ $2.30000 t-01 * 1.49840 t+00$ $2.40000 E-01 * 1.50408 E+0 C$ $2.500001-01 * 1.511378+00$ $2.600001-01+1.51847 t+00$ $2.80000 t-01+1.53344 t+00$ $2.90000 \mathrm{r}-01 * 1.54208 \mathrm{E}+00$ $3.00000 t-01 \quad 1.550 E 7 E+0 C$ $3.10000 t=01 * 1.55960 E+00$ $3000 \mathrm{E}-1.5080 \mathrm{LL}+00$ $3.30000 E-01+1.57443 t+00$ $3.40000 \mathrm{E}-01$ 1.50831E 3.5000 T. $3.60000 t-01 * 1.60893 E+00$

$1.6 \mathrm{~L} 134 E+C D \quad 1.1144 B t+C U$ 1. $58947 E+C O$ $1.574<E t+C$ 1. 5 YULE+CC $1.560 \mathrm{E} L \mathrm{~L}+\mathrm{CO}$ $1.5,2 \mathrm{E}$ UE + CO 1.5450 LE C CO 1.319. Cli + CO $1.534,11 E+C O$ 1.52445: + CD $1-525$ ser $+C D$ 1. $325 \mathrm{set}+\mathrm{C}$ $1.52241 E+C O$ 1. 1 B et $+C 0$ 1. 51 / 200 1. $3172 \mathrm{JE}+60$ 1.5171 $10 E+C D$ 1.5171 aE CD. 1.5 $514 E+C C$ $1.52117 E+C O$ $1.52447 E+C O$ 1. $52774 E+C O$ $1.53134 E+C O$ . $535 \mathrm{EBE}+\mathrm{CO}$ $1.540 \% 2 E+C O$ 1. $540 C D E+C O$ 1. $55105 E+C O$ 1. 5 b $11 E+C O$ 1. $5 t 4 b 2 E+C 0$ 1. 571 YQE + CO 1.57 $190 \mathrm{COE}+\mathrm{CO}$ $1.5674 \mathrm{SE}+\mathrm{CO}$ 1.595 8 日E + $C O$ $1.60447 \mathrm{t}+\mathrm{CO}$ $1.61132 E+C O$ 1. $62241 E+C O$
$1.1027(t+C)$ $1.64717 t+C U$ $1.07203 i+c u$
$1.05917 t+c u$ 1.t.16 $12 i+c$ $1.63521 t+c c$ $1.624111+00$ 1. $51529 t+c$ $1.00071 \mathrm{c}+\mathrm{C}$ 1.00b7lctc 1. $54963 L+C$ 1. $\$ \$ 631 \mathrm{~B}+\mathrm{C}$ . $50122 t+C u$

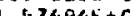
1. JTaAe + Co 1. $373472+C$ 1. $57076 c+C 0$ 1. $26 \mathrm{HB1t}+\mathrm{CO}$ 1. $36758 k+C$ 1. $56767 t+C$ $1.56724 \mathrm{~L}+\mathrm{CO}$ 1. $36808 E+C O$ 1. $56957 \mathrm{E}+\mathrm{C}$ $1.57168 \mathrm{E}+\mathrm{Co}$ $1.5144 \mathrm{CL}+\mathrm{CO}$ $1.577716+C O$ $1.5815 \theta E+C O$ 1. $58602 \mathrm{C}+\mathrm{CU}$ 1. $59091 t+C$ 1. $59644 t+C 0$ 1. $59644 t+C O$ $1.00240 E+C O$ $1.60884 E+C U$ $1.01574 E+C O$ $1.63 C B \mathrm{CE}+\mathrm{CO}$ $1 .+3403 t+C$
1. $85_{2} 243 t+00 \quad 2.00114 t+00$ I. 43 UNURE +00 1. $6 \mathrm{COB} 84 \mathrm{E}+0 \mathrm{O}$ 1. I8d) $1 E+U O$ $1.77021 \dot{c}+v 0$ 1.75270t+00 1. $736 j / E+O C$ 1. $72117 t+0 C$ $1.807165+00$ $1.09411 \mathrm{E}+00$ 1.03411E+00 1. 6 H $214 E+O C$ $1.47131 E+C 0$ 1. 1. G44GJE+OD 1. $64403 E+00$ I.A3764E+DD 1.63150e+00 $1.62637 \mathrm{E}+00$ $1.622 \mathrm{U} 4 \mathrm{E}+00$ L. $61854 E+00$ 1. $D 15 B 5 E+00$ 1. $013+5 E+00$ $1.012 \mathrm{~d} 2 \mathrm{E}+00$ $1.61243 E+00$ $1.61275 E+00$ $1.41377 \mathrm{E}+00$ $1.61540 E+00$ $1.0 L 7 \mathrm{E} 1 \mathrm{E}+\mathrm{O}$ $1.62078 \mathrm{E}+0 \mathrm{D}$ $1.62436 E+00$ 1.62051E+Q $1.02831 E+Q 0$ l. $3854=00$ $1.64434 \mathrm{E}+0 \mathrm{O}$ $1.64434 E+00$ L. $\$ 5745 E+00$
1.97231t+uU $1.94446 t+00$ $1.91405 t+00$
$1.84455 t+00$ 1. $89455 t+00$ $1.84963 E+C 0$ $1.82 \times 25 E+C L$ $1.81 U 11 E+00$ $1.742245+0$ $1.79224 t+00$ 1.77360 + $1.745001: 00$ $1.743701:+00$ 1.12090t+00 $1.70989 L+00$ $1.70004 E+00$ $1.69123 t+40$ $1.68342 \mathrm{t}+u$. $1.67658 E+40$ $1.67009 E+U R$ $[.66573 t+00$ $1.60165 E+00$ 1. $65845 t+00$ 1. $656085+00$ $1.65453 \mathrm{E}+0 \mathrm{O}$ $1.63317 E+00$ $1.653775+00$ $1.05451 t+00$ .6545it +00 $1.65297 E+00$ .6581 LE+DO $1.664+2 t+00$ $1.66445 t+00$ $1.07 j 111+00$ $1.61435 E+00$
$2.100021+00$ $2=130221:+00$ $2.0961 C E+C$ 2. U⿺ $3 \in 3 F+C$ $2.03278 t+C$ $2.00351 t+C l$ 1.47 $7 \mathrm{HE}+\mathrm{CO}$ 1. $444586+c 0$ $1.9<48 t E+C$ $1.40160 \%+C$ $1.401601:+C$ $1.87 \times 7 y+c$ $1.65328 t+C 0$ $1.84017 \mathrm{t} C$ $1.82<38 t+c$ 1.80500 is 1. 1.7retit + ct $1.76378 E+C i$ 1. 3 's? $11 \mathrm{~L}+\mathrm{C}$ $1.74157 E+C O$ $1.73212 \mathrm{E}+\mathrm{CO}$ $1.7 ? 375 E+C 0$ - $11640 E+C O$ 1.71007E+00 1.7047 let00 1.70U $29 E+C$ $1.09679 t+C 0$ $1.67418 E+C O$ $1.09243 \mathrm{E}+\mathrm{CO}$ $.69151 E+C O$ $1.09139 E+C O$ $1.64346 E+C O$ $1.67359 E+C 0$ $1.69841 \mathrm{E}+\mathrm{CO}$
1.
$2.347 E 2 E+06$ 2. $2 u \cdot 24 t+01$ 2.?C¿TEL+00 $2.22315 t+0 t$ $2.10 \leq \leq 15+00$ $2.1493 \mathrm{E} i=0 \mathrm{C}$ Z.1IELEitci

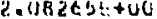
2.051 is $3+40$ 2.0ंe 2 tet +40 $1 . y+3=10 ;+00$ $1.465131:+06$ $1.944) \mathrm{CE}+\mathrm{OV}$ $1.12177 \mathrm{t}+00$ $1.900 .2 \mathrm{t}+00$ 1. POC 3GE +UL 1. $Q$ G $\in E i+O C$ 1. $\because 44432+L C$ 1. $.205 \mathrm{CE}+\mathrm{CL}$ 1. $1387 i+40$ 1. $8005 C t+60$ 1.7HE35E + 00 $1.1774 \mathrm{CE}+O \mathrm{C}$ 1. $7676 C 0+04$ 1. 75A42E +00 $.75: 33 E+00$ - $7393 C E+O O$ $1.7347 E F+00$ 1. $73122 E+00$ $1.73122 E+00$
$1.72859 E+0 U$ $12 \in 8<E+0 D$ 1. $1268<E+00$ 1. $7255 t E+0$ I. 725 Eetuo 1. 72673 to l. $72826 E+00$

2. $54624 E+00$ $2.49475 E+00$ $2.44534 E+L C$ C. $35 B O 1 E+U O$ 2. $35274 E+00$ $2.3 C 918 E+U D$ $2.2682 C E+W 0$ $2.15583 E+00$ U 2. OC44 BE+ 0 1. $47025 E+00$ 1. 1.J $561 E+00$ $1.9352 \mathrm{E}+60$ 1. $85322 E+00$ $1.87615 E+00$ $1.85987 E+00$ - $14494 E+20$ 1. $B ? 134 F+00$ $1.01401 E+00$ 1. $\theta C 794 E+00$ $1.78936 \mathrm{E}+00$ l. $77533 E+U O$ 


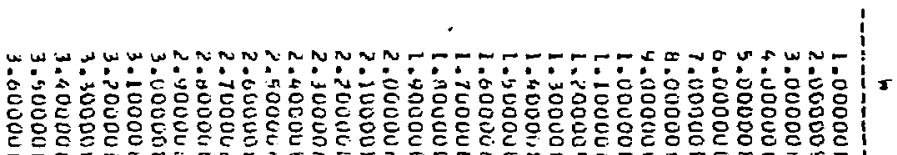
의

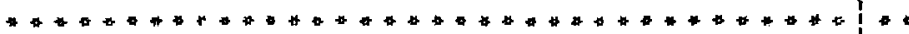

-

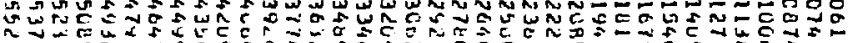

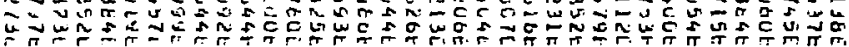

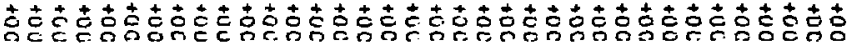

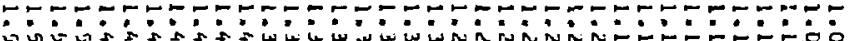

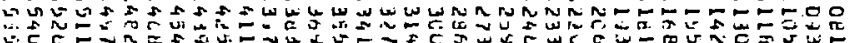

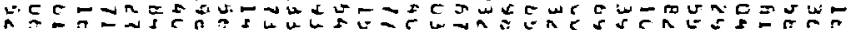

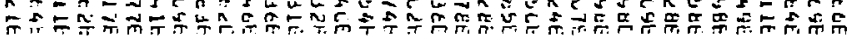

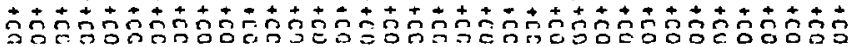

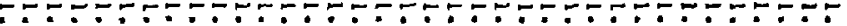

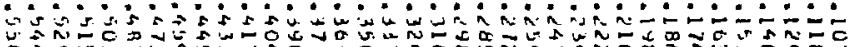

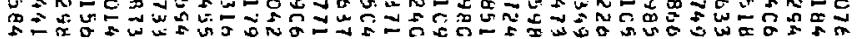

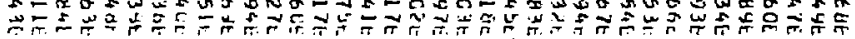

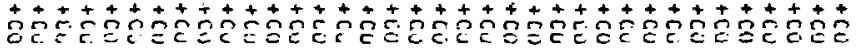

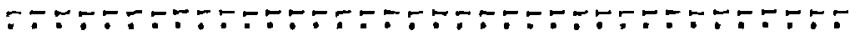

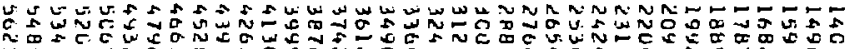

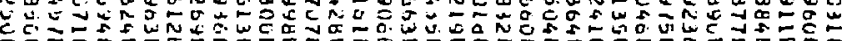

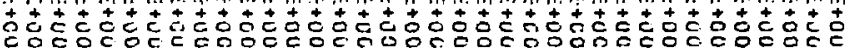

"is

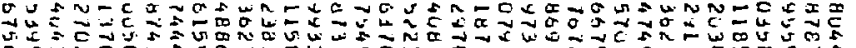

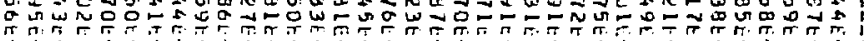

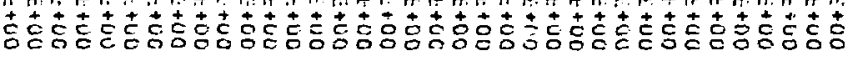

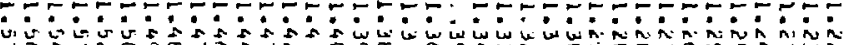

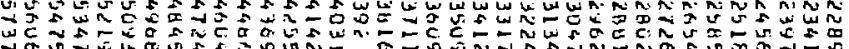

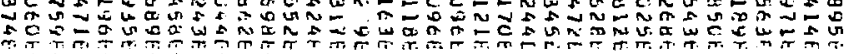

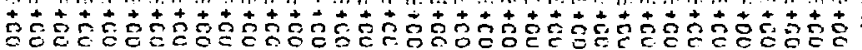

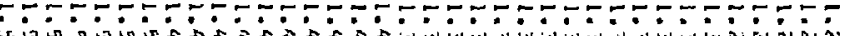

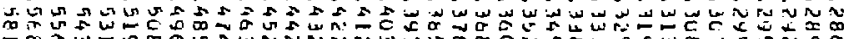

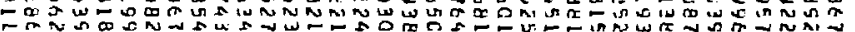

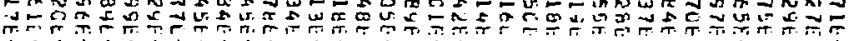

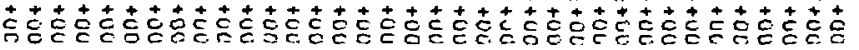

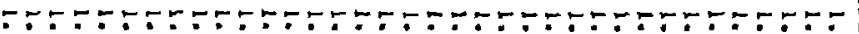

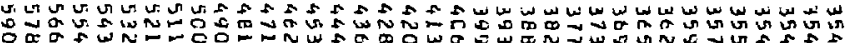

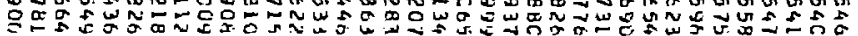

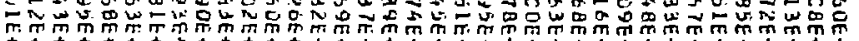

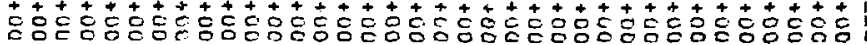




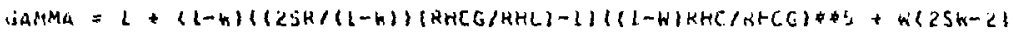

$$
S R=1.3 \mathrm{C} \quad \mathrm{BHCL}=2.6 \mathrm{C} \quad S \mathrm{~W}=1.7 \mathrm{C}
$$

RHC $1.00000 C-02 \cdot 1.43347 E+U C$
$2.00000 C-02,1.42940 K+0 C$ $3.00000 t-02+1.424 v 2 t+00$ $4.00000 t=02 * 1.42311 t+00$ 5.0000 1.421 15k+00 $6.00000[-02+1.419 \mathrm{det}+0$ $7.00000 \mathrm{r}-02$ 1.41902E+00 a.00000r-0z $9.00000 \mathrm{t}-02$ $1.00000 \mathrm{t}-01 * 1.420 \mathrm{l}$ bt $+0 \mathrm{C}$ $1.10000 E-01+1.4216 B E+00$ 1. $.0000 t-01+1.42374 E+120$ $1.30004 t-01+1.42633 t+00$ $1.40000 E-01+1.42943 E+D O$ $1.50000 \bar{i}-01 * 1.433 i 3 t+00$ $1.600005-01 * 1.43712 t+00$ $1.70000 \mathrm{E}-\mathrm{Cl} * 1.44167 \mathrm{E}+00$ $1.40000 \mathrm{E}-0 \mathrm{l}$ 1.44Ge 1.900001-01 1.452 $141+00$

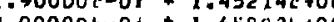
2.0000 $1.458 c 2 t+00$ 1. $1.40432 \mathrm{~L}+0 \mathrm{C}$ $2.20000 t-01$ 1.47203t $+0 \mathrm{C}$ 2.30000-O1 1.nrel2E+00 2.40000 E-O1 1.48554E 400 $2.50000 r-01$ 1.45343t+0C $2.60000 \mathrm{~F}-01+1.50162 E+D D$ $2.70000 E-01 * 2.51015 t+0 D$ $2.80000 E-01$ 1.51901E+OO $2.90000 E-01+1.52 \theta 19 E+0 C$ $3.00000 E-01 * 1.537 a 7 E+00$ $3.10000 E-01+1.54745 E+00$ $3.20000 E-01+1.55752 E+00$ $3.30000 E-01 * 1.56785 E+00$ $3.40000 E-01 * 1.57445 t+00$

1.9LULUE+CO 2.UDCGLE+LU 2.10000E+00 2.20000E+UC

$1.52413 E+C C$ $1.51062 E+C 0$ . $5 \mathrm{C}$ a $73 E+C O$ $1.50225 t+C$ $1.446,342+C$ $1,491 \mathrm{CECO}$ $1.4 E 74 C E+C O$ $1.4 \mathrm{USZF}+\mathrm{CO}$ $1.48116 t+C$ $1.479 C \mathrm{dE}+\mathrm{CO}$ $1.47707 E+C O$ $1.47652 t+C D$ 1.476 LLE +CO $1.4773 L E+C O$ $1.47840 E+C O$ $1.4906 \mathrm{BE}+\mathrm{CO}$ $1.4 E 233 E+C$ $1.42233 E+C$ $1.44314 E+C 0$ $1.48046 E+C$ $1.4467 C E+C C$ 1. 4 C 1.50t bEE + CO 1. SOE beE+CO 1. $518<6 E+C O$ $1.52551 E+C O$ 1. 532 L $7 E+C O$ 1. $540 C 4 E+C$ 1.547t $\mathrm{EE}+\mathrm{CO}$ l. $556 \mathrm{LUE}+\mathrm{CO}$ 1. $564 \angle B E+C O$ 1. $5736 \mathrm{CEE}+\mathrm{CO}$ 1.562 \& $B E+C O$ $1.59243 E+C D$ $1.60231 E+C O$ $1.01249 E+[C D$

\section{$1.02735 t+60$} $1.81464[+C O$ $1.60254 E+60$ $1.59224 E+C O$ $1.51242 E+C J$ 1. 5 ग 54 Et 1. $55545 t+c 0$ $1.53827 t+C O$ $1.55193 \mathrm{t}+\mathrm{CU}$ $1.34641 E+C 1$ l. $54184 L+C 0$ $1.53773 t+\mathrm{Cu}$ $1.53454 t+C L$ 1. $5320 \mathrm{et}+\mathrm{CO}$ $1.33 C 34 E+C C$ $1.52930 E+C O$ $1.42893 t+C y$ $1.32893 \hbar+C$ $1.53 C 15 E+C$ $.53 C 15 t+C$ $1.531696+C$ 1. $33658 E+C O$ $1,539 t 7 t+C O$ $1,53967 E+C 0$ 1. $54372 \mathrm{t}+\mathrm{C}$ $1.54609 E+C$ $1.35298 E+C$ 1. $55 \mathrm{t36E}+\mathrm{C}$ $1.56422 E+C$ $1.57654 E+C 0$ $1.57731 E+C$ 1. $3845 I E+C O$ $1.59213 t+C 0$ $1.00014 E+C$ $1.60854 E+C O$ $1.01731 E+C O$ 1. $62644 E+C O$

\section{$1.143 H a t+00 \quad 1.67432 t+00$} $.72010 E+00 \quad 1.85092 t+00$

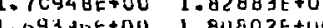
$1.67452 t+00 \quad l .78 t 46 t+60$ 10 . 1.66614 Etco $1.17012 \mathrm{E}+00$ 1.053 retoo 1.75R9E+100 $1.64245 E+00$ R. $1.6321 \mathrm{CE}+\mathrm{CO} \quad 1.7222 \mathrm{CE}+\mathrm{OOO}$ $1.02271 E+00 \quad 1.70850 L+40$ $1.6142 j E+00 \quad 1.6 .1268 t+00$ 1. $5067 L E+00$ 1.68433E+UO L. 6CUUGE+CE $1.67302[+60$ $1.59427 t+00 \quad 1.66431 t+00$ $1.56933 E+00 \quad 1.65579 E+U 0$ $1.58520 \mathrm{E}+0 \mathrm{C} \quad 1.64623 \mathrm{E}+40$ .5 1. $5793+5+00$ 1.64 1.57y 5 E $1.63586 \mathrm{e}+00$ 1.57 $547 E+00$ 1. $1.62702 E+00$ 1.57647e+00 $1.627032+100$ 1. $7646 E+00$ 1.62

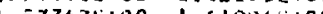

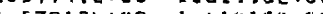
- $57912 E+00$ 1. $1.61915 E+00$ . $58140 E+001.61908 E+U 0$ 1.58422E+00 $1.61473 t+00$ $1.5877 \mathrm{TE}+00$ 1.62106E+00 $1.591 \mathrm{~L} 1 \mathrm{t}+00 \mathrm{O} 1.62306 \mathrm{t}+00$ 1. 39041E+00 $1.62371 E+00$ $1.60153 E+00 \quad 1.62898 E+00$ $1.60717 E+00 \quad 1.63285 t+00$ $1.6133 G E+00 \quad 1.63730 E+00$ $1.61490 E+00 \quad 1.64231 E+00$ $1.62696 E+00 \quad 1.64786 E+00$ $1.63446 E+00$ $1.64239 E+00$

\section{$2.02+33 E+C 0$} $1.44973 E+C G$ 1. $461 E 2 E+C O$ $1.43498 E+00$ $1.909765+C 0$ $1.88594 t+04$ 1. $66357 E+C 0$ $1.84250 t+00$ $1.82275 \mathrm{EE}+\mathrm{C1}$ 1. $80427 \mathrm{E}+\mathrm{C}$ 1. 7 H $7 \mathrm{C} E \mathrm{TE}+\mathrm{CC}$ $1.771075+C 0$ 1. $75627 \mathrm{E}+00$ $1.742641:+C 0$ 1. $73015 E+C$ $1.71876 t+C$ 1. $70946 E+C$ $1,64+205+C 0$ $1.690968+C O$ l. . $744 E+C O$ $1.07209 E+C$ $1.057665+C O$ $1.05+111+C O$ $1.05411 \mathrm{t}$ $1.85955 E+C 0$ $1.65849 \mathrm{t}+00$ 1. 65 DCCE+CO 2. $05867 E+0$ $1.65980 E+C O$ 1. $66176 \mathrm{E}+\mathrm{CO}$ 1. $66433 E+0 Q$ $1.66756 \mathrm{E}+00$ 1. $67142 E+C 0$ $1.675 \mathrm{e} E E+00$ 1. $E A 093 E+C O$
$2.11 \times 39 t+0 c$ $\begin{array}{ll}2.17+35 t+0 L & 2.35474 E+00 \\ 2.14303 E+00 & 2.31126 E+00\end{array}$ 2-2E949E+UO 2 - 4 CCCE+COU $2.19153 E+U$ 2014 $3 E+00$ 2.155z7E+UD 1.

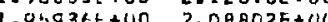
$1.934150+0 \mathrm{C} 2.05696 \mathrm{E}+00$ $1.3104 \mathrm{NE}+00 \quad 2.02755 E+00$ . $868<2 E+00$ 1.99977E+00 1. $96735 E+610$ 1.97357E+UO $1.647 \mathrm{HSE}+0 \mathrm{U} \quad 1.94893 E+50$ 1. A296EE+UO $1.92574 E+00$ $1.01261 t+00 \quad 1.40414 E+40$ $1.797 a 1 \mathrm{~L}+\mathrm{LU} \quad 1.8 \mathrm{P} 393 \mathrm{E}+100$ $1.76285 F+00 \quad 1.864135+00$ $1.70965 t+00$ l. $04709 E+00$ $1.75711 \mathrm{t}+00$ 1.83160E+00 1.74E $1.13713 E+C D$ l. $B C 327 E+00$ $1.72840+00$ 1.700JE+00 1.7C0 1.71 1 .

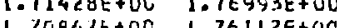
1. $1080 \mathrm{VE}+00$ 1.76112E+00 $1.704 C 3 E+00$ 1.75341E+00 $1.70031 E+00$ I.74675E+00 1.C9748E+OC 1.74113E+OD - AYSa3E+UO 1. $73650 E+00$ $1.69441 E+00 \quad 1.73264 E+00$ $1.69411 E+00$ 1.7301UE+00 $1.644 E C E+00 \quad 1.72827 E+00$ $1.69584 \mathrm{E}+00 \quad 1.72731 E+00$ $1.64781 \mathrm{E}+00 \quad 1.72719 \mathrm{E}+00$ $1.70048 E+00 \quad 1.72789 E+00$ $1.703836+00 \quad 1.72936 E+00$ 
GAMHA $=1+(1-W)(125 R /(1-h))(H H C G / R H()-1)((1-W) H A C / K H C G)+\$ 5+W(25 H-Z)$
$S H=1.3 \mathrm{C}$
KHCS $=2.0 \mathrm{C}$
SW $=1.70$

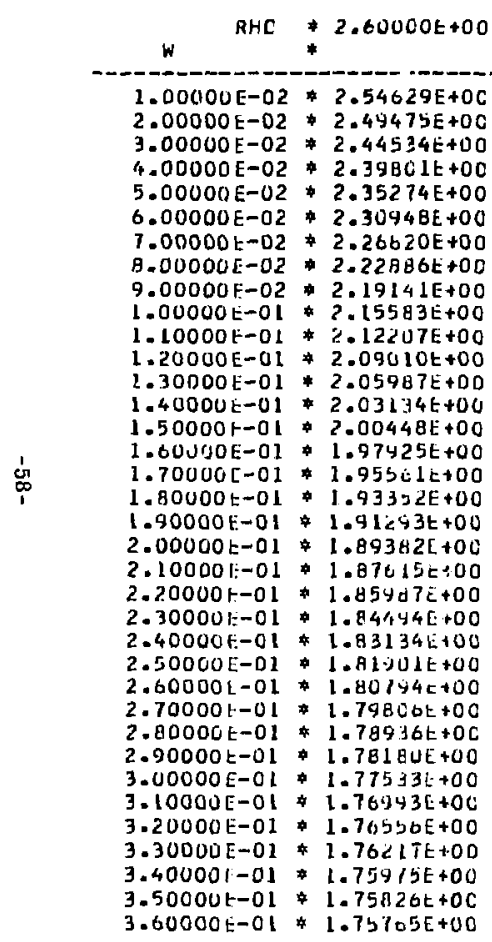


$00+310285^{\circ} 1$ $30+306 h \angle 5 \cdot 1$ $00+396275$ "I $00+322.155 *$ $00+7896 E 5 \cdot 1$ $00+35 E B C 5^{\circ}$ $00+\exists E Z L Y 5=1$ $20+\exists$ EE9JS-I $00+324565 * 1$ $00+3 \rightarrow 25 B \Rightarrow+1$ $00+\exists L J 5 \angle b-i$ $00+361596=1$ $00+315555^{\circ} \mathrm{I}$ $00+3519$ $00+3 L O L E{ }^{\circ} 1$ $00+\exists B 2 B 2 b=1$ $00+3196 T+=1$ $00+\exists \zeta \sqcup \mid$ โ

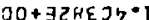
$0 D+\exists \varepsilon E Q B E=$ $00+\exists A$ I $38-$ $0 n+\exists \operatorname{tha\varepsilon }$. O)+ $00+3655 L E \cdot T$ $00+79667 \varepsilon^{\circ}$

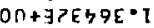
$27+36.265 E \cdot 1$ $30+38$ ch5 1 $00+7606$ DE $r n+7 b t G h<-1$

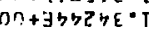
OO+ ᄏT TSEE I I $00+79895 E \cdot 1$ $00+\exists \cap 8+E F$. $0 D+\exists \& Z E E E=I$ $0.3+3612 \varepsilon \mathrm{E}-\mathrm{I}$ $0 \cap+\exists \angle O T E E$.
$00+328115 \cdot 1$ $00+765 \mathrm{RSG} \cdot 1$ $00+3 Z \gg S 75$ ० I OO+38EZES०I $0:+37 h 615 \cdot$ $00+719705$. $00+723564 \cdot$ 004315184.1 00 स $1694-1$ $03+2459540$ 20+78女口少・ DOH उООहE" 1

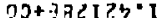
$031782600^{\circ}$ $O J+\exists L E B G E \cdot$ DO+JDZLdE DJ+ TE? गJ+3दमदवह $0 J+368 \mathrm{SCE}$ : $0 O+A \rightarrow \operatorname{Sn} B \cdot$ OJ+tIbDEE. OJ+t CO+ 315 IEE OS+75BDIE. OD+ JEלEOE $00+7 \angle 296 ? .1$ OOS $37 E \angle 95 \cdot 1$ $02+17=0120$ $03+17 E 012$ $00+335 ? 92=$

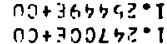

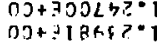
OD+ 7ด6?E?-1 $0 J+73 \geqslant 925 * 1$ $0 D+36,5027 \cdot 1$ $00+j z$ Ehl? $00+\exists \bar{C} T 995^{\circ} 100+3 \bar{C}+195^{\circ}$ $00+70+755 \cdot 1$ $00+79 \angle R E 5^{\circ}$ $00+722575^{\circ} 1$ $00+38<T / 55^{\circ} \mathrm{I}$

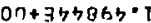
$00+70258+=1$ $00+71.0 ? \angle 2=1$ $00+70 n \times 5 b=1$ $20+7919$ मक 1 $00+76$ E. F. $00+7 P L \cap 7 \rightarrow=1$ $00+7 \geq 2804=1$ $00+7 \rightarrow B S, 6 E=$ $0 \cap+719 F 8 E$. $00+725$ TIE. 0 ON+ 78GSGE $B O+\exists O P \& D E \cdot 1$ $00+38$ โवEE"I $D O+\exists ट L \neg Z E=1$ O०स दरकराहै D)+JSE?DE OD+ZESIG? $00+\exists 0 \angle 092 \cdot$ $00+3 \angle 101.2 \cdot$ $00+\exists$ मधद दर

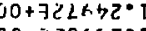
$00+j z B: i 85 \cdot 1$ $00+7 \varepsilon$ TOEZ $00+789052=1$ $00+35 \% 112$ $00+3 \angle b 20 Z=1$ $00+\exists \varepsilon 2 E 6 I^{*}$ $00+732581^{\circ}$ $00+\exists Z \cap 2 L T$ * $0 n+390691^{\circ}$ OO+FEELלद. OD+JOEGIS 1 cot.j61,505.? $03+380164=1$ O० (5) $00+38<05$. $0+\exists Z E L E h+1$ + $356 E 27=1$ $00+3890$ Ib $30+\exists 05 \angle 6 E=1$ $0 \cap+\exists$ カタBE・ $00+3 B \rightarrow 1 L E \cdot 1$ $00+\exists E S R S E \cdot 1$ $00+3685+E$. OO+ ЗРZEEE $0 D+76 \angle O R E=1$ $30+\exists$ 2 $B O E=$ $00+3 \leq 156=1$ $00+5$ वरद $00+30178 ?=$ $00+\exists \rightarrow 12 L ? \cdot 1$ $00+\exists$ EFOQ $00+3 \angle 9 B+2=$ $00+7912 E ?$ ? $00+\exists z 8522 \cdot 1$ $00+j द$ ThI $00+724 E D 2 \cdot 1$ $00+j 11.26 T^{\circ}$ $00+\exists$ I I ZE I * $00+359121$. $00+\exists \rightarrow \varepsilon 191^{\circ}$ $00+3$ ZIS 1 . $D O+\exists+E I+I *$ DO+JS9IET - I

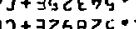

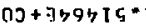
$02+71$ b 00 * 0כ+ คว+ $05+36585+$ (1) 0ง+ 了+ Оว+ $03+\exists \zeta 520 n-$ $10+753348=1$ OJ+JZZSLE" $0 J+3 \forall 979 E=1$ $0 J+\geq 123+\varepsilon^{\circ}$ OJ+ ЭСBमEE OJ+ J十STटE. OJ+7ЬEBOE : $0 J+7 n 256 ?^{\circ}$ $0 J+3 \varepsilon z 26 z=1$ $03+75 E 592=$ $90+7 E 5952 \cdot 1$ $0 J+7$ EREด * $0 J+752$ IE? $0 J+78<8\lfloor ? *$ $03+7 h>902 *$ $00+112061 \cdot$ $00+711281$ * $53+7 \rightarrow 15<1=1$ $0 J+70 E 84 I^{-}$ $93+709947=1$ ก)+ उ־OदEI I OJ+3ZवEZI I $03+\exists$ SEZT I* ด丁 $+7 \rightarrow 2101=$ 0) +3 ह $30 \rightarrow 5$. $0 J+35$ क 25 $03+316015^{\circ}$ $00+33+06$. $00+3+61$ $0 J+3 \geq 5 \angle 8 n=1$ $00+7$ IES $00+3$

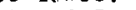
$03+\exists 4 द$ करम० $0+\exists z 196 E^{\circ}$ $03+\exists 0 \cap Z$ ZE 1 $0 J+\exists C 5 \angle T E=1$ O+ ЭT KE SE $0 J+\exists 7 K G E E=$ $0 J+\exists 7 J 9 Z E \cdot$ $0 J+\exists E ? Z 1 E \cdot T$ $0 J+\exists L$ B $03+70<$ ८ $05 \cdot 1$ $03+7=1112 \cdot 1$ $03+339<52=1$ $03+\exists \varepsilon 1$ म 2 * $0 J+\exists \varepsilon L O \varepsilon \tau^{\circ}$ $n כ+31+L I 2 * 1$ $00+38$ I OJ+ $\exists E \cap I \in I \cdot ?$ $03+\exists \angle E L L I * 1$ $0 J+\exists J ว द \nabla 1 * 1$ $0 J+$ JE I टदI $0 \mathcal{0}+3$ CEGE T-I $03+7 \angle 9927 \cdot 1$ $03+367+11 \cdot 1$ $0 J+325[0.10$ $00+797690-1$ $0 J+3 J 0 \angle L O 0^{\circ} 1$ $00+\exists \forall E Z 5 S=1 * 10-300009 \cdot E$

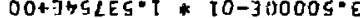

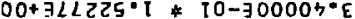
$00+\exists \varepsilon 0205 \cdot 1$ \& $10-700005=6$ $00+715$ ESh"1 $10-70000 z \cdot 10$ $00+320$. T $00+350$ AL' $00+396 e^{\circ} 1$ *

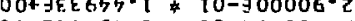

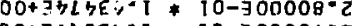
$30+3 L 10 Z Z^{\circ} 1 * 10-70000 L=2$ $00+349505^{\circ} 1 \neq 10-300009^{\circ} 2$ $00+3 द 1 I b E=1 * 10-700005 \cdot 2$ $00+760016=1 * 10-30000+10=5$ $00+79229 E \cdot 1 \neq 10-30000 \varepsilon \cdot 2$ $00+30 B \angle B E \cdot 1 * 10-700002 \cdot 2$ 00+395FEE"T * 10-300001'Z 00+7LZGIE*I * 10-100000" J0+7Z050E"T $\% 10-300006 * 1$ $00+328062=1 * 10-300008=1$ $30+=\angle 9912 * I * I 0-70000 L * 1$ $00+395202=1$ 10-300009:1

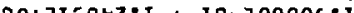
$00+10-30000$ - I $00+3$ s

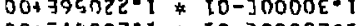
$00+\overline{9} \angle 5902 * 1 * 10-300002 \cdot 1$

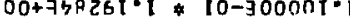
$00+3 L 06 L 1 \cdot 1 * 10-7000,17 * 1$ $00+75 E 591 \cdot 1+2 n-300000 \cdot 6$ $00+3 L L T E T \cdot T+20-300000-8$ $00+76$ LET-1 $20-300000 \% 2$

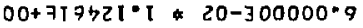
$00+321111 * 1 * 20-300000 \cdot 5$ $00+708 \angle 60^{\circ} \mathrm{I}+20-300000^{\circ}+2$ $00+305480 \cdot 1+20-300000 \cdot 8$ $00+38 Z 120^{\circ} 1 * 20-300000 * 2$ $00+7 E 1850^{\circ}$ \& $20-700000 \cdot 1$ 
IAMMA $=1+(1-h)(12 S R /(1-h))(R H C G / K H C)-1)(1)-h) R H C / R+[E)+5+w 125 h-2)$

$$
S K=1.3 \mathrm{C} \quad \text { *HCO }=2.05 \quad \mathrm{Sn}=1.16
$$

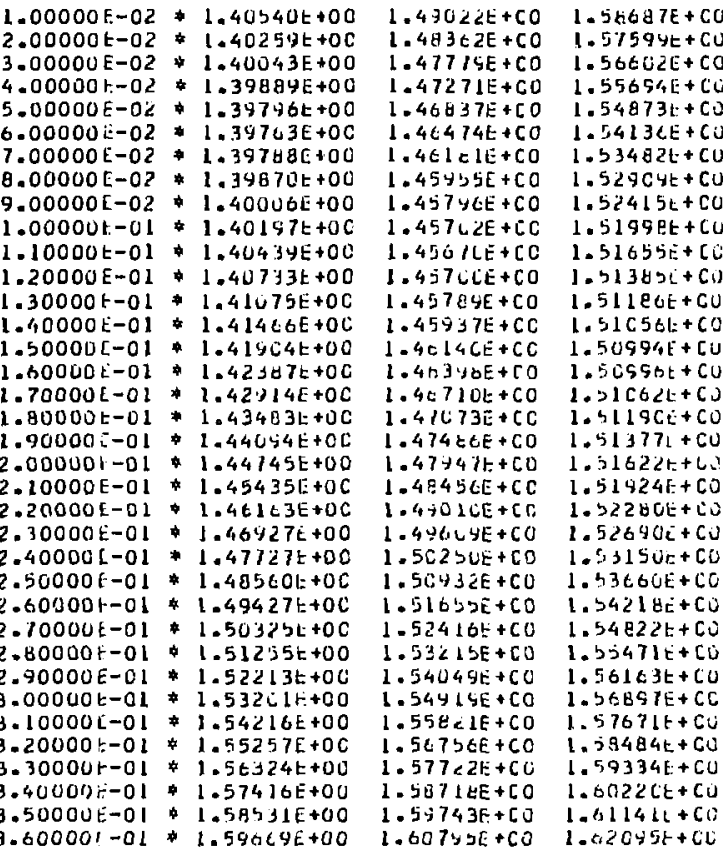

$1.6960 a \dot{E}+00$

$1.6960 \dot{E} E+00$ $1.02039 E+00$ $1.6651 \mathrm{CE}+\mathrm{CO}$ $1.65221 \mathrm{t}+00$ $1.63300 \mathrm{H}+00$ $1.42811 E+00$ $1.01752 E+00$ $1.50799+00$ 1.5918E+00 1. $59137 E+00$ 1. $58444 E+00$ 1. $57437 E+00$ $1.51314 t+00$ 1. $56871 E+C O$ $1.565 \cup 8 E+U C$ $1.56222 \mathrm{E}+00$ $1.56011 E+C O$ 1. $5507 \mathrm{~d}+00$ $1.55805 E+00$ 1. $558 C$ G +00 1. $55873 E+00$ 1. $3600 \mathrm{DE}+\mathrm{CC}$ 1. $56200 E+0 C$ 1. SACOCE+OC 1. $56456=+00$ $1.57141 \mathrm{E}+0 \mathrm{O}$ 1. 57 41Etac $1.57567 i+00$ 1. $58046 E+00$ 1. $38576 E+0$ I. $591 \supset O E+C C$ 1. $59784 E+00$ $1.6 C 458 E+00$ $1.01176 \mathrm{t}+0 \mathrm{C}$ $1.61950 E+C O$ $1.02740 \mathrm{E}+0$ L. $63382 E+00$
2.20UUCl:+10 $2.30 C G L E+C O \quad 2.40$ CCCE +00

$1.81843 t+00$ $1.74 / 44 E+C 0$ $1.17709 E+60$ 1. $75 y 13 t+00$ 1.74170E+UD $1.74176 E+00$ . 12335e+00 $1.64547 t+00$ L 6 B 35 5 $6 E+00$ $1.67170 t+C 0$ $1.66 \cup 87 \mathrm{~F}+00$ $1.65103 E+00$ $1.642\lfloor 7 t+00$ $1.03425 i+00$ $1.62726 i+00$ $1.62117 t+40$ $1.6151,60+00$ $1.611+9 t+0 \mathrm{C}$ $1.60805 t+00$ 1. 60 , $12 E+00$ $1.60137 t+00$ $1.60<17 t+00$ $1.60<170+00$ $1.601711++00$ $1.60196 t+00$
$1.602491+00$ $1.602491+60$
$1.645,50+00$ $1.004501+00$ - 6 Un 75 to $1.60 \cdot 3 \mathrm{~F}+40$ $1.6 ! 311 L+00$ $1.61718 t+00$ $1.62 l b 1 t+00$ $1.6 \% 0 y p t+6,0$ $1.63 \ddot{2} c 7 t+00$ .63 ist 7E+CO $1.64536 t+00$ $1.652716+00$ $1.454585+c 0$ $1.42774 t+C O$ $1.40231 \div+00$ $1.07 b 27 t+c 0$ 1. $81558 t+i$ $1.03422 \mathrm{C}+\mathrm{CO}$ $1.81415 t+60$ 1. $7953 t E+00$ $1.7772 \mathrm{CE}+\mathrm{CO}$ $1.76145 E+C$ $1.746295+C 0$ $1.73<28 \mathrm{E}+\mathrm{CO}$ 1.71939t + $1.7016 \mathrm{CL}+\mathrm{CO}$ 1. $.+1+2 \mathrm{e} L+\mathrm{CL}$ I. 6 A7?CE + CO 1. $67654 \mathrm{t}+\mathrm{Cl}$ 1. $67085+C 0$ I. BSal I It +CU 1. $55834 t+C 0$ 1. $65346[+C O$ $1.0494 \mathrm{hLCC}$ $1.04030 t+c 0$ $1.64246 \mathrm{~F}+\mathrm{CC}$ 1. $641 \% 1 E+C O$ $1.04172 k+C$ $1.6424 t\}+00$ $1.6434 i E+C O$ 1.04 EC2L+CL $1.640801+C 0$ $1.65222 \mathrm{E}+00$ $1.05624 \mathrm{~F}+\mathrm{CO}$ 1. $660261+00$ $1 . \operatorname{teth} 4 t+C 0$ $1.67177 \mathrm{~F}+\mathrm{CO}$
$2.105 c 2 t+00$ ?. $) 7178 E+00$ $2.04018 t+00$ $2.01 C L 1 E+00$ $1.981 E C L+00$ $1.9346 C L+U L$ $1.32508 t+00$ $1.405 C C 1+00$ 1. $88235 x+0 i$ 1. Bulctitou 1. PAl I Ll + Ou $1.82<541+0 C$ $1.40522 \mathrm{t}+0 \mathrm{C}$ 1. IOS1EL+OL $1.77433 r+0 u$ 1. $700 \mathrm{CegE}+00$ 1. $142 \bar{a} 1 \mathrm{~L}+\mathrm{LiC}$ 1. $736 b c t+b t$ $1.726625+0$ 1. $717451:+00$ $1.70932 t+6 u$ $1.702231+00$ $1.09 t C E \mathrm{C}+U \mathrm{C}$ 1. 0 gected $1.68665 \mathrm{t}+\mathrm{OC}$ I. fia $329 l+00$ 1.td0e1t+00 $1.67417 i+00$ $1.61834 \mathrm{E}+06$ 1.4.7ejuttuo $1.679621+0$ 1. $680485+00$ l. $6132621:+00$ l.5855CE+UU 1. OC 9CCE +UU $1.6+315 t+U C$
2. SCCCUE+OO $17018 E+00$ $2.230 \cup 2 E+\cup 0$ 2. $19163 E+00$ $2.15509 E+00$ 2.12 C25E+00 2. $05566 E+00$ C. $02584 E+00$ 1. $9781 E+00$ $1.97 C O 6 E+00$ $1.94583 E+40$ 1. $4222 \mathrm{UE}+U 0$ 1. 9 CCC4E+UU I. $479.3 D E+00$ 1. $85946[+00$ 1. $84117 F+00$ $1.132531 \mathrm{E}+U \mathrm{U}$ 1. $8 C 996 E+00$ $1.755 d 4 t+00$ 1.7B245E + UO $1.17125 E+U C$ $1.16072 E+00$ 1.151 'SOE +00 1. $74298 F+00$ $1.73572 \mathrm{E}+0 \mathrm{C}$ 1. $1244 \cup E+00$ - I2 Y4YE+ UO 1. $11048 E+00$ - 1194ae+00 $1.71422 t+00$ $1.71266 E+00$ 1.711 4 3E +UO 1. $11205 E+00$ . 71 G4 + +00 


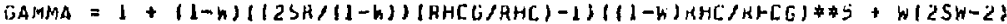
$S R=1.30$
RHES $=2.65$
$S W=1.76$

W

*2.600lar +oc

$.00000 t-02 * 2.45043 t+00$

$2.00000 \mathrm{E}-02 * 2.402 \mathrm{blt}+00$
$3.00000 \mathrm{E}-02 * 2.35721 \mathrm{E}+00$

$3.00000 E-02 * 2.35721 E+00$
$4.00000 E-02 * 2.31360 E+00$

$4.00000 E-02 * 2.3136 U E+00$
$5.00000 E-02 * 2.27143 E+00$

5.DONOOE-02 $* 2.27143 E+00$

$6.00000 \varepsilon-02 * 2.23217 t+0 C$

7.000001:-02 2.19428E+00

$8.0000 U E-02+2.15823 \mathrm{E}+00$

$9.00000 \mathrm{E}-02 * 2.22397 E+0 \mathrm{C}$

$1.00000 \mathrm{E}-01+2.09146 \mathrm{E}+00$

$1.10000 E-01+2.06071 E+00$

$1.20000 \mathrm{E}-01+2.031 \mathrm{E} 3 \mathrm{E}+0 \mathrm{O}$

$1.30000 \mathrm{~L}-01 * 2.00419 \mathrm{E}+0 \mathrm{C}$

$1.40000[-0 I+1.97837 E+U C$

$1.50000 E-01+1.95411 E+00$

$1.60000 E-01+1.93140 E+00$

$1.700001-01 * 1.91018 E+00$

$1.80000 E-01+1.890425+00$

$1.90000 E-01+1.87208 E+00$

$2.00000 E-01+1.85514 E+00$

$2.10000 E-01+1.83254 E+00$

$2.20000 E-01 \times 1.82525 t+00$

$2.30000 E-01 * 1.8122 b E+00$

$2.40000 E-01+1.8004 B E+00$

$2.50000 E-01+1.78992 E+00$

$2.60000 E-01+1.78453 E+00$

2.70000E-0L $1.77227 E+00$

2. $80000 E-01 \div 1.76511 E+00$

$2.90000 \mathrm{E}-01 * 1.75902 \mathrm{E}+00$

300001.75390500

3.0.000E-01 *1.75396e+00

$3.20000 \mathrm{E}-01 * 1.74680 \mathrm{E}+0 \mathrm{O}$

$3.20000 E-01 * 1.74680 E+O D$
$3.30000 E-01 * 1.74464 E+00$

$3.40000 \mathrm{E}-01 * 1.74337 \mathrm{E}+00$

$3.50000 E-01 * 1.7429 B E+00$

$3.60000 E-01+1.74342 t+00$ 
GAMMA $=1+(1-H)((25 R /(1-n))(A H C G / R H C)-1)((1-W) R H C / A H C G) * 05+W(2 S H-2)$

$5 H=1.30 \quad$ HHCG $=2.70 \quad$ SW $=1.76$

$1.00000 E-02 * 1.05516 E+00$ $2.00000 \mathrm{E}-02 \div 1.06845 \mathrm{E}+00$ $3.00000 \mathrm{E}-02 \div 1.081 \mathrm{~B}+\mathrm{LE}+0 \mathrm{O}$ $4.00000 \mathrm{E}-02 * 1.09524 \mathrm{E}+00$ $5.00000 \mathrm{E}-02+1.10873 t+00$ 6.00000E-02 *1.12230E+00 $7.00000 \mathrm{E}-02 * 1.13593 \mathrm{E}+00$ Q.00000E-02*1.14962E+00 $9.00000 E-02 * 1.16337 E+00$ $1.00000 E-01 \div 1.17714 E+00$ $1.100008-01$ 1.10106t+00 1.10000 Ea $1.20000 \mathrm{E}-01$ 1.20498E+0 $1.30000 \mathrm{E}$. 1.21 (1) $1.40000 \mathrm{E}=01 \quad 1.23300 \mathrm{E}+00$ 1.50000E-01 1.24708E+0 $1.60000 E-a 1+1.2612 \lambda E+00$ $1.70000 E-01$ * $1.27539 E+00$ $1.80000 \mathrm{~F}-0 \mathrm{~L}+1.28962 \mathrm{E}+00$

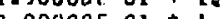
2.00000E-01 1.3182UE+OC $2.10000 E-01 * 1.33256 E+0$ $2.20000 E-01$ 1.34646E+0 $2.300001-01 \neq 1.36139 E+00$ $2.40000 E-01 * 1.37586 E+00$ $2.50000 \mathrm{E}-01 * 1.39037 \mathrm{E}+00$ $2.60000=01 * 1.40491 E+00$ $2.70000 E-01 \& 1.42449 t+00$ 2. $80000 E-01+1.43410 E+00$ $2.90000 E-01 * 1.44873 E+00$ $3.00000 E-01 * 1.46340 E+00$ $3.10000 E=01$ 1.478 IUL+OO $3.200005-01$ 1.4928 3. 1.50757E+00

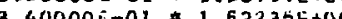
3.40000 E 01 1. $3.60000 \mathrm{t}-01 \geqslant 1.55197 \mathrm{E}+00$

$1.07275 E+C O$ $.0852 \mathrm{OE}+\mathrm{CO}$ $1.0977 \mathrm{EE}+\mathrm{CO}$ $1.11642 \mathrm{E}+\mathrm{CO}$ $1.12317 \mathrm{E}+\mathrm{CO}$ $1.136 L 3 E+C 0$ 1. $14 B 47 E+C O$ 1. $162 \angle U E+C O$ $1.17513 E+C O$ 1. $16833 E+C O$ - IE 1. $20162 E+C$ . $24495 E+C$ $.22843 E+C$ $1.25535 E+C O$ $1.26921 E+C O$ $.28245 E+C O$ . $29674 E+C O$ . $310 C 1 E+C O$ $.324535+C O$ 1. $3389 L E+C O$ 1. $35255 E+C O$ 1. $36005 t+C O$ 1. $38080 E+C O$ 1. $39 B O O E+C O$ $1.40925 E+C D$ $1.42355 E+C O$ $1.43790 \mathrm{E}+\mathrm{CO}$ $1.45229 E+C O$ $1.46672 E+C O$ $1.48119 E+C O$ $1.4957 C E+C O$ 1. $51026 \mathrm{E}+\mathrm{CO}$ $1.52484 E+C O$ 1. $53946 E+C O$ $1.5541 \angle E+C O$

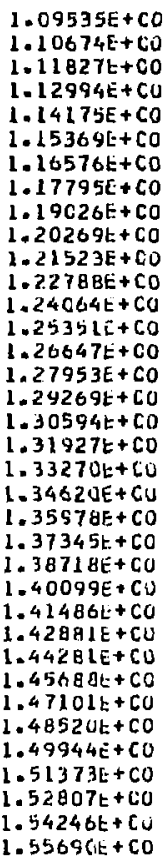

$1.09535 E+C O$
$1.10674 E+C O$ $1.11827 t+C 0$ $.14175 \mathrm{SE}+\mathrm{CO}$ $.15369 t+C 0$ $1.17795 E+C O$ . $19 \mathrm{C2} 2 \mathrm{E}+\mathrm{CO}$ $1.20269 E+C O$ $1.2278 B E+C O$ $1.26647 E+C 0$ 1.30594t+CO $1.31927 t+C D$ $34620 E+C U$ $57 \mathrm{dE}+\mathrm{CO}$ $40099 E+C O$ $.41486 t+C O$ $1.442 \mathrm{BLE}+\mathrm{CO}$ $1.45 h 8 d e+C O$ $1.48520 \mathrm{Ut}+\mathrm{CO}$ $1.54246 t+C u$ 1. $55696 \mathrm{CE}+\mathrm{CO}$

\begin{tabular}{|c|c|c|}
\hline $\begin{array}{l}13378 E+00 \\
14404 E+00 \\
1544 B E+00 \\
16510 E+C 0 \\
17570 E+00 \\
18687 E+00 \\
19800 E+00 \\
20930 E+00 \\
22076 E+00 \\
23237 E+00 \\
24412 E+00 \\
25602 E+00 \\
26806 E+00 \\
28024 E+00 \\
29255 E+00 \\
30498 E+00 \\
31734 E+00 \\
33021 E+00 \\
34300 E+00 \\
35541 E+00 \\
36492 E+00 \\
38203 E+00 \\
39524 E+00 \\
40855 E+00 \\
42196 E+00 \\
43545 E+00 \\
449 C 3 E+00 \\
46270 E+00 \\
47044 E+00 \\
49026 E+00 \\
50416 E+00 \\
51612 E+00 \\
53216 E+00 \\
14626 E+C 0 \\
56043 E+00\end{array}$ & 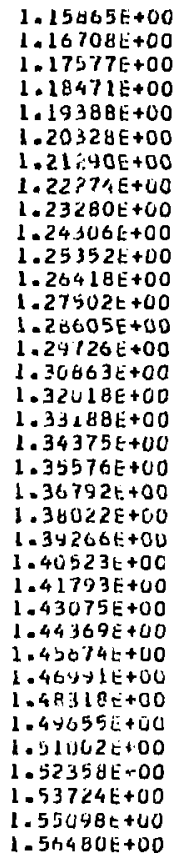 & 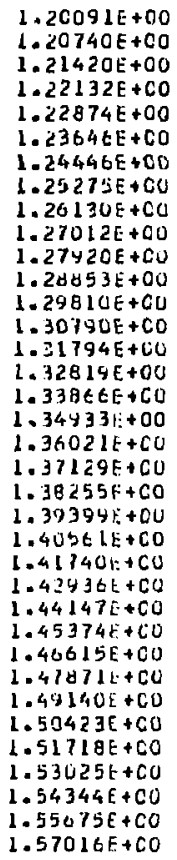 \\
\hline
\end{tabular}

1. $60000 E+00$

1.251 $31 E+U 0$ $1.25549 E+00$ 1. $260 C E E+00$ 1. $26503 E+U 0$ I. $27038 t+00$ 1. 2760 E $+O C$ $1.28218 t+00$ $1.288615+00$ 1. $29538 \mathrm{E}+00$ $1.29538 E+00$
$1.30249 E+00$ 1. $30952 t+00$ 1. $31767 \mathrm{E}+00$ 1. $31767 E+00$ 1. 334075 $1.342715+00$ 1. $3427 \mathrm{te}+00$ $1.35162 E+00$ 2. 3CRCCE+ 1. $37 C 25 E+00$ 1. $3799 E E+U C$ 1. $3 \forall 591 E+O C$ 1. $400 C 9 E+00$ $1.41 C 5 I E+U 0$ $1.42116 \mathrm{t}+00$ $1.432 \mathrm{C}$ LE +UO 1.44 ? $2 E+00$ l. $45435 t+00$ $1.465816+00$ $1.4774 t t+00$ $1.409250+00$ l. $501291+00$ $1.51340 t+00$ $1.525755+00$ 1. $32575 t+00$ 1.550911+00 $1.550911:+00$ $1.50365 t+00$ 1.57E.61itoc

$1.31003 E+00$ 1. $11210 E+00$ 1. $31407 E+0$ 1. $31652 E+00$ 1. $31944 E+00$ 1. $32282 \mathrm{E}+00$ 1. $33560 E+00$ 1. $34070 E+00$ 1. $15209 \mathrm{E}+00$ 1. $5505 E+00$ 1. $36494 E+00$ 1. 3449 प 00 1. $77933 E+40$ 1. $B T O O E+0$ 1. $145010^{+}+0$ 1. $1.41196 \mathrm{E}+00$ $1.4208 B E+00$ $1.43007 E+00$ $1.44934 \mathrm{E}+00$ L. $46462 E+00$ $1.48 C 13 E+00$ 1.490 प $8 E+00$ 1. 5 C $285 E+00$ - $51303 E+00$ 1. $52443 E+00$ $1.53603 E+00$ 1.547 HE + UO l.55979E+ 00 $1.52255 E+40$ $1.52427 E+00$ 
GAMMA $=1+(1-h)((2 S K)(1-h))(K H[G / K H C)-1)(11-h) K H(K / K+[G) * \$+W(2 S W-2)$

$$
S R=1.3 L^{\circ} \quad H H C G=2.7 \mathrm{G} \quad S W=1.76
$$

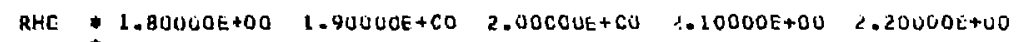
$2.300006+C U \quad 2.40 C O C E+00$

2. 5 COOUE +00

\begin{tabular}{|c|c|c|c|c|c|c|c|c|}
\hline $\begin{array}{l}.00000 \mathrm{E}-02 \\
.00000 \mathrm{E}-02 \\
.00000 \mathrm{E}-02 \\
.00000 \mathrm{E}-02 \\
.00000 \mathrm{E}-02 \\
.00000 \mathrm{E}-02 \\
.00000 \mathrm{E}-02 \\
.00000 \mathrm{E}-02 \\
.00000 \mathrm{E}-02 \\
.00000 \mathrm{E}-01 \\
10000 \mathrm{E}-01 \\
20000 \mathrm{E}-01 \\
30000 \mathrm{E}-01 \\
40000 \mathrm{E}-01 \\
50000 \mathrm{E}-01 \\
60000 \mathrm{E}-01 \\
70000 \mathrm{E}-01 \\
80000 \mathrm{E}-01 \\
90000 \mathrm{E}-01 \\
00000 \mathrm{E}-01 \\
10000 \mathrm{E}-01 \\
20000 \mathrm{E}-01 \\
30000 \mathrm{E}-01 \\
40000 \mathrm{E}-01 \\
50000 \mathrm{E}-01 \\
60000 \mathrm{E}-01 \\
70000 \mathrm{E}-01 \\
80000 \mathrm{E}-01 \\
90000 \mathrm{E}-01 \\
00000 \mathrm{E}-01 \\
10000 \mathrm{E}-01 \\
20000 \mathrm{E}-01 \\
30000 \mathrm{E}-01 \\
40000 \mathrm{E}-01 \\
50000 \mathrm{E}-01 \\
60000 \mathrm{E}-01 \\
\end{array}$ & 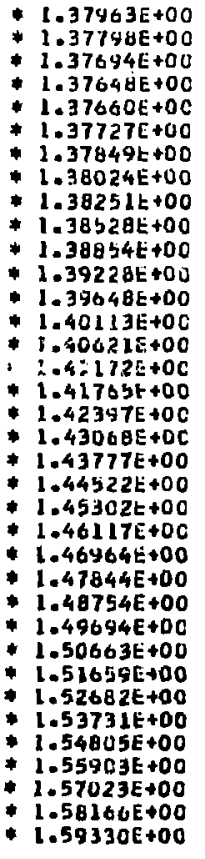 & 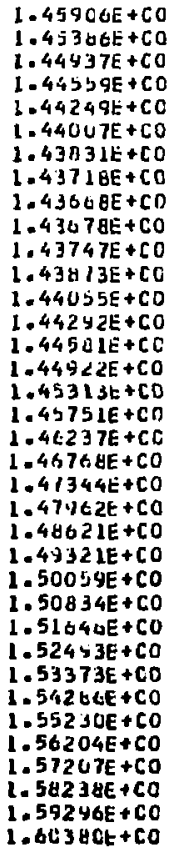 & 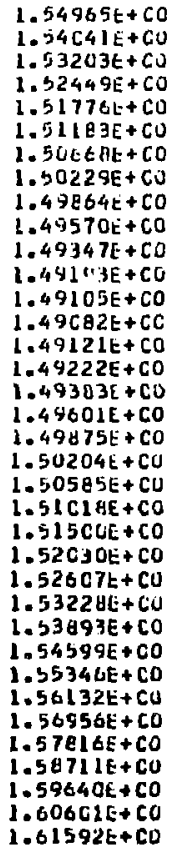 & $\begin{array}{l}1.652 U 7 E+00 \\
1.63832 E+00 \\
1.62557 E+00 \\
1.61381 E+00 \\
1.60300 E+0 D \\
1.59313 E+00 \\
1.58418 E+00 \\
1.57611 E+00 \\
1.56892 E+00 \\
1.56257+00 \\
1.55705 E+00 \\
1.55234 E+00 \\
1.54641 E+00 \\
1.54525 E+00 \\
1.54283 E+00 \\
1.54113 E+00 \\
1.54013 E+00 \\
1.53982 E+00 \\
1.54017 E+00 \\
1.54116 E+00 \\
1.54278 E+00 \\
1.54501 E+00 \\
1.54712 E+00 \\
1.55120 E+00 \\
1.55313 E+00 \\
1.55959 E+00 \\
1.56457 E+0 C \\
1.57000 E+00 \\
1.57601 E+00 \\
1.58243 E+00 \\
1.58430 E+00 \\
1.59660 E+00 \\
1.60431 E+00 \\
1.61243 E+00 \\
1.62093 E+00 \\
1.62980 E+00\end{array}$ & $\begin{array}{l}1.76695 E+00 \\
1.74319 E+00 \\
1.73059 E+00 \\
1.7113 E+00 \\
1.64078 E+00 \\
1.68452 E+00 \\
1.67133 E+00 \\
1.6517 E+00 \\
1.64802 E+00 \\
1.63786 E+00 \\
1.62867 E+00 \\
1.62042 E+00 \\
1.61308 E+00 \\
1.60663 E+00 \\
1.60146 E+00 \\
1.59632 E+00 \\
1.59242 E+00 \\
1.58930 E+00 \\
1.54697 E+00 \\
1.58519 E+00 \\
1.58454 E+00 \\
1.54440 E+00 \\
1.54496 E+00 \\
1.58618 E+00 \\
1.58804 E+00 \\
1.59054 E+00 \\
1.59363 E+00 \\
1.54132 E+00 \\
1.60157 E+00 \\
1.60636 E+00 \\
1.61169 E+0 \\
1.61752 E+00 \\
1.62384 E+00 \\
1.63064 E+00 \\
1.63789 E+00 \\
1.6455 A E+00\end{array}$ & 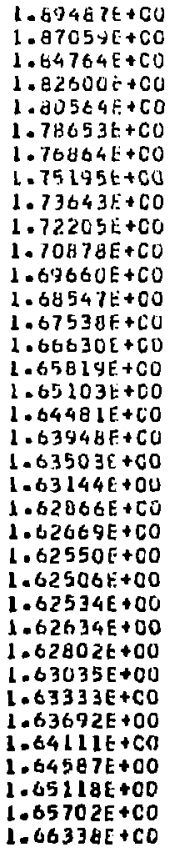 & 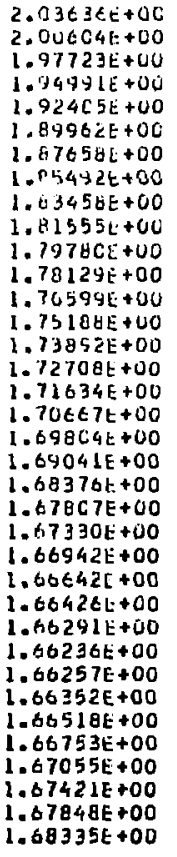 & 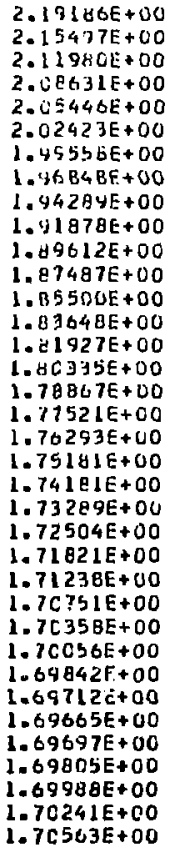 \\
\hline
\end{tabular}




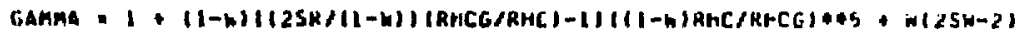
SA $=1.36$
AHCG $=2.70$
$\sin =1,76$ n

AHE 2.60000E.00 2.7UOLEL.5O

$1.00000 E-02$ :.341/4k+00 2.54624E*CO

$2.00000 t-02: 2.31738 E+00$ 2. $244415 t .00$

$1.00000 E-02$ - 2.27373t+50 2.44JJ4E 400

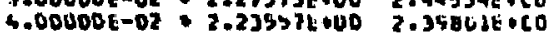

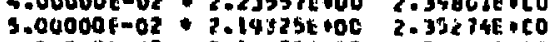

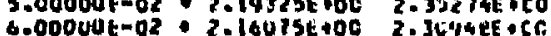

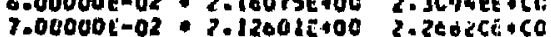

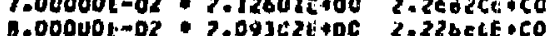

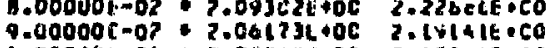

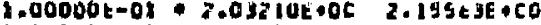

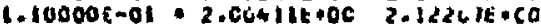

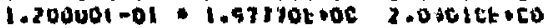

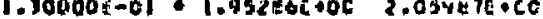

$1.400001-01 \cdot 1.924534006 \quad 2.03154 E+C 0$

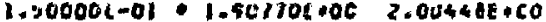

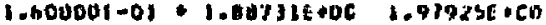

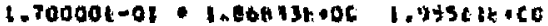

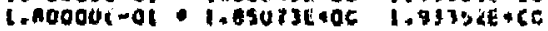

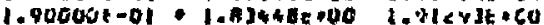

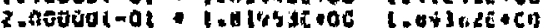

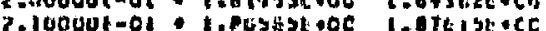

.

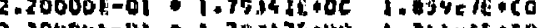

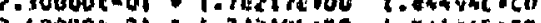

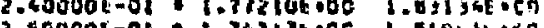

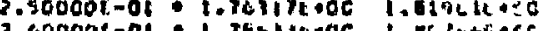

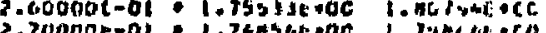

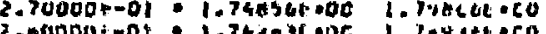

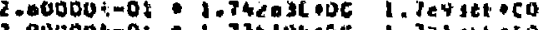

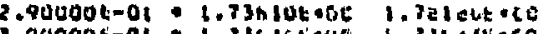

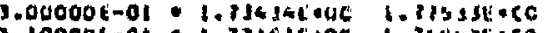

$3.100006-01$. 1.73ISIE+0C 1.164+3E-60

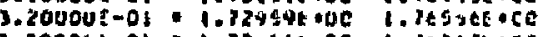

$3.30004-01 \cdot 1.720546 .06 \quad 1.15217 t+C 6$

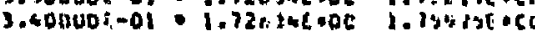

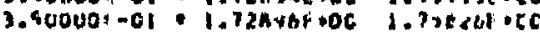

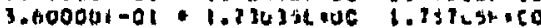




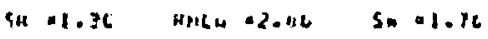

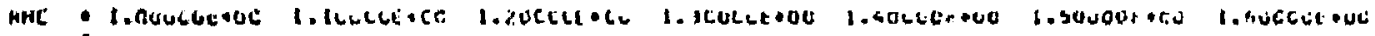

1. Tcuuar:*uo .

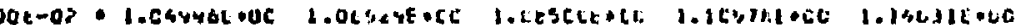
.0000Gl-0z:1.067ityctac

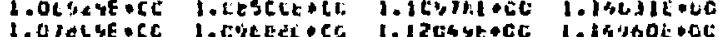

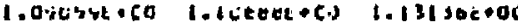

1.150111 .00

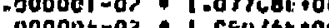

s.00000i-c?

1.

1.

$1.171: 16 \cdot 601.381+16 \cdot 06$

$1+723 \cdot 1, \cdot 60$

1.1424 cteor

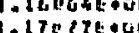

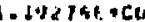

$1.16701+004$

1.776470 .00

s.t.coser

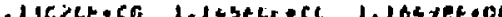

1.

1.2E02 1E. OC

$7.00040 x=02+1.132075 .06$

1.

i icerleco ielenifceus

ave

1. 3 EUTJEFUD

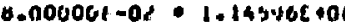

$9.0000 \mathrm{Ll}-02$ 1.130VUE $+0 \mathrm{C}$

$1.600001 ;-01 \div 1.11349 t+0$

$1.10000 \mathrm{t}-01+1.167+3 \mathrm{t}+0 \mathrm{c}$

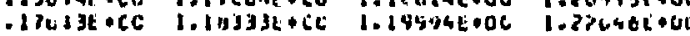

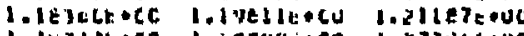

-

$1.20352 E \cdot 00$

19991600

$.2 .235 c \cdot 06$

1.

$1.3 C 14350.0$

tht

1. 110210000

1.30000r-01 1.216201000

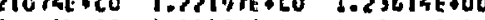

1.253641 .00

1.

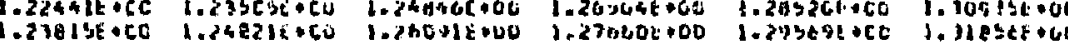

$1.31 \operatorname{cs} 36+60$

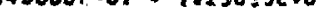

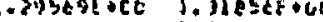

ShiE •U

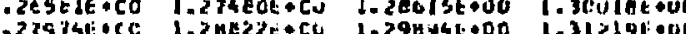

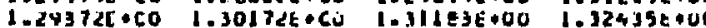

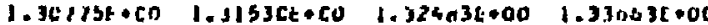

$1.32164 E+C O \quad 1.32694 E+C d \quad 1.337+3 t+00 \quad 1.34 \% 08 t+00$

$.300361+60$

. 120catou

1.3)

-2003t.CC 1.147ettelio

1. $35304 E \cdot 00$

1. Je 1 STE. 00

$1.70000101 \cdot 1.213 .01 .00$

$1.90000 \mathrm{r}-0)$
1.800001

7.0000Ui-01 1.31634E+0C

2.10000t-CS 1.33udCE $\$ 0 C$

$2.200005-01 * 1.345345000$

33og6tica

I. $37644 E+00$ 1.

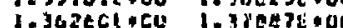

1. $174500 \times 00$ I. 3EBOAE OO 1. 35 MIAE OO

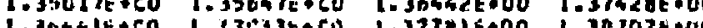
1. Trastion

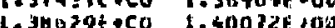

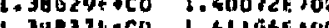
$2.30000 i-01 * 1.35 \% 44 t+00$ $2.400001-01+1.37441 t+00$ $2.50000 \mathrm{r}-01 \div 1.36400 \mathrm{CL}+00$

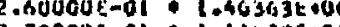
$2.70000 i-01 \cdot 1.414245+10$ 2.80000t-al 1.432 .06400 $2.90000 \mathrm{E}-01 \cdot 1.447695 .0 \mathrm{C}$ $3.000001:-01 \cdot 1.46243 E+00$ $3.1000 a t-01: 1.473140+00$ $3.20000 \mathrm{t}-01 \cdot 1.491486+00$

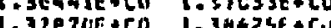

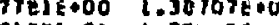

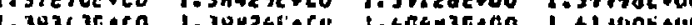
1. L

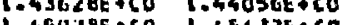
$1.454172,60$ $1.4321 \mathrm{HE}+00 \quad 1.43436 E 40$ $1.445976000 \quad 1.452698+60$ 1.4lus 5 . $C 0$ $1.4 \sum, 9 a t \cdot 00$

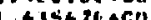
446321000 lotecest oce 1.411745000 $1.42325 i+40$ $1.4347460 . \mathrm{J} 0$ J. 444161000 I. As sest:00 1.410756000 $1.40 \geq 0 J 6.00$ 1.45753600 $\{-4 T 3\}$ af + Co $1.466805 \cdot+C D$ $1.499421 * 00$ I. 503860.00 $1,41,07 E 000$ 1. $4 \geq 0 \mathrm{~T}, 0 \mathrm{00}$ 1.42 46636000 1.44 Or DE 00 $1.44926 E+00$ $1,46005 E+00$ $1.472160 \% 00$ $1.482476 * 00$ $1.43493 \mathrm{t}+\mathrm{C}$ . 507 seteco $1.49397 E+00$ .30000 al 1.504795400 $3.40000[-01-1.52142 e+00$ 1.44446E-CO 1.44772t+CO whr ritedo I. SI HITE +CO J.32632E *CO

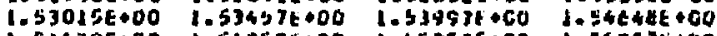
$3.500006-01$ 1.53647t*UC 1.53846E+CO 1.541G4t+CO 1.54439E*00 1.54E40E+00 1.353525000 l.555ste*00 1.524615000

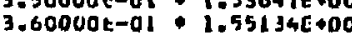

1. 553 SIE oco 1.55809E+00 t. 50250C

$1.36116 \mathrm{E} \cdot 00$

$1.54677 E+00$

1.5744 IE +00 


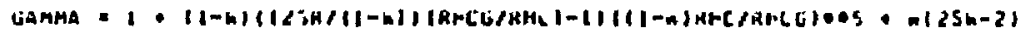

SK $=1.7 \mathrm{~L}$ NHCO $=2.8 \mathrm{C}$ Sh 1.10 H

1.0irVOP $-02 \div 1.334121:+0 \mathrm{C}$ $2.04000 \mathrm{r}-02 \cdot 1.33453 E+0 \mathrm{C}$ $3.00000+-02+1.3351,7 E 000$

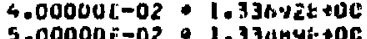
n.000aof-02 1.341 $15 t+0$

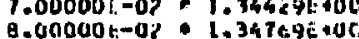

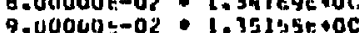
$9.00000 \div-02$ l. 1.000001-01 1. 35spat 00 1.10000r-01 1.34uedioc 1.2000ut-cl 1.3637SE

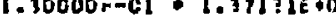
$1.40000:-01+1.37727 \mathrm{~L} 000$ $1.50000[-01 * 1.303 t 2 t+0 c$ $1.600005-01 \cdot 1.35034 i+0$ $1.70000 \mathrm{t}-0 \mathrm{l}: 1.3971 .2 \mathrm{t} 40$ $1.600001=01$ 1.404neston $1-40000 t-01$ I.4t2ezt+00

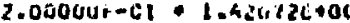
$\left.2.10006+01+1-4 t^{2}\right) 15 t+00$

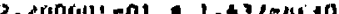
2.

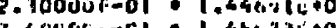

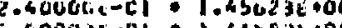
-

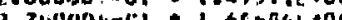

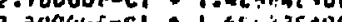
2.

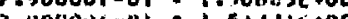

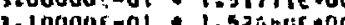

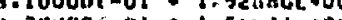

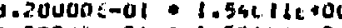
1.300001:-01 - 1.5314:28.0c $3.40000 r=01 \cdot 1.563936 .0 C$

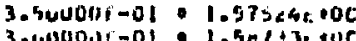
2. ICOUuetuin 7.20Nude.00

L. A. T4.JE:CO $1.401<3 t+C 0$ $1.3+314 E+C D$ -3)7LGL \&CC - Jis jitice 1. Juthsebe? i. 34 IEAt ice .3 is luk +ce 1. Arilesfict $1.4013<1 \cdot C C$

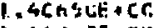
$1.461 .7 F \cdot C 0$ $1.415 .221 .0 \mathrm{CD}$ 1.41 P $145+20$ $1.42]<C[\bullet C C$ $1.42 t+1 \mathrm{IC}+C 0$ $1.414<4 t+60$

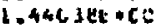
lombriatice I.AS J the +CO lone lite eco 1. he liet ico I. Ariter aco 1.4राust. $1.4 \pi 3<15.00$ $1.439361+C$

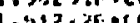
1.312 a $21: 00$ 1.521 in 3r+co - $31672 k+c$ $1.3+1<y+4 C$ $1-53253 E+C$ 1. 5 hl LLLECA $6.57344 E=C O$ $1.5 \pm 511 E+5 C$ $1.3 \times 0=6 E+C C$

\section{$1.9637 \mathrm{EK}+\mathrm{CL}$} $1.41141 E+C$ $1.4784) \mathrm{L}+\mathrm{CO}$ 1.4671 ur $+C 6$ 1.463Ci:t + CL $1.444641 . C 0$ 1.45 oust oc 1.4569 ist o co 1.4548210 co $1.4535 h t+20$ $1.452 \mathrm{C} 11$. $1.43272 t$. CO $1.4432 A C=6:$ $1.43+311+60$

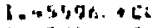
$1.45 ! I \mathrm{HL}_{L}+\mathrm{Cu}$

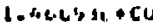
$1 .+1 \times 421 t+c 0$ $1.46795 \mathrm{~L}$ + CO $1.472278+C u$ $1 .+11 \mathrm{i}$ h + ci $1.4 \mathrm{H2} 2 \mathrm{H}_{1}+\mathrm{CL}$ 1.4t 19 th 0 l. 4 Hif at ? Ca 1. Docb 1. 1. Tolset cc 1.3148 1. $2225 \mathrm{rt}+\mathrm{Cr}$ - sicantecu 1 -3jschr+Co 1. 24 ve I. 3 icsut + cu 1.540420 . 1. ".7blact 00 $1.5001 .0 t+C_{2}$ 1. 34 is $11+56$

\section{I-3inlictoco} $1.56300 t+00$ $1.55+178+00$ 1. B45U2t+UC I. SJ日LILECo 1.53124E000 1. $22510 \mathrm{k}=00$ 1. $31+116000$ 1. FLS41t+OL $1.311006+00$ I. Scenteco i. SCG 1St+UO 1. PUA I $3 E+00$ I. je $141-000$ 1. Sestict $\$ 00$ i. sOsabeco 1.504 the 0 1. $300436+C 0$ 1. 506tici $+c i$ 1. 51$\}$ set $-\mathrm{CE}$ I. Slatas!.cC $1.71647 \mathrm{E} 000$ 1. $322024+0$

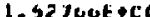
ar - b3c गerd $1.94503 t+00$ $1.551720 \mathrm{CC}$ 1. SEgdate00

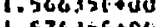
$1,5742 \mathrm{OE}+\mathrm{CC}$ 1. 3H2'SE+t'O 1.591 $\angle 6 E 000$ 1 . $b$ CO2 I $t+00$

I. GCYjSE+CL I. Al $472 L+00$

\subsection{1 .00} - obuh 1t kd 1.61 IA IAL +UL: $1.62<0<2.00$ bl $1-$ hucsit-co 1.5.) S111:000 - besile 40 - stribitun 6.5717 It 60 - Snuzinute

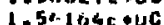
1.5s 104: 000 I.524731.0uO 1.03241600 $1.550821 \cdot 6 C$ $1.54113 \mathrm{t}+00$ $1.54 \times 73 t \cdot 00$ $1.53 u 2 c t+u c$ $1.551 .11 t \cdot 00$ in 110 ceca - S Julleco - Sristiol ola - 50 - Shlo sri ol - sesure - storna Throta 2. Strlerteco $i .543606+c 0$ $1.60 u k 71.00$ $1 . \angle 0090 E+00$ 1. $611+1+6+00$ 1.62 '. $19 t+60$ $1.63 .102=000$

\begin{tabular}{|c|c|c|}
\hline 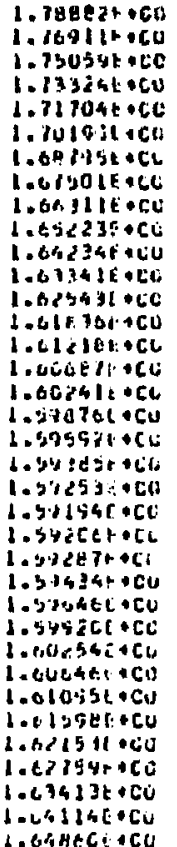 & 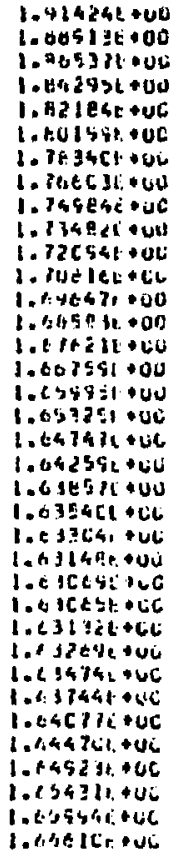 & 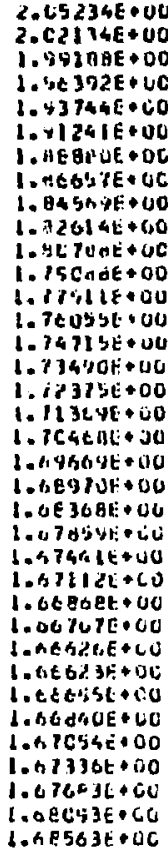 \\
\hline
\end{tabular}

$2.4000 c+000$ 2.5COCOE+00 boststcoto

$1.65583 t+0 C$ 

SH 1.1 .30
KHLG $=2.80$
$\operatorname{Sin}=1.10$

$\rightarrow$

$1.0000 U E-02: 2.20331 t+00 \quad 2.366 L \theta E+C O \quad 2.54624 E+C U$

$2.00000 E-02 * 2.16614 E+00$ 2.3236bE+CO $2.47475 E+C O$

$3.00000 E-02 * 2.13144 E+0 C$ 2.2a13CE+CG $2.44534 t+C C$

$4.00000 E-02+2.09634 t+00$ 2.24114E+CO $2.398 C 1 E+C C$

$5.00000 E-02: 2.06425 E+0 C \quad 2.2 C 254 E+C O \quad 2.35274 t+C O$

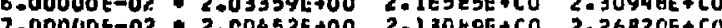

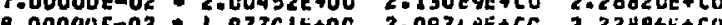

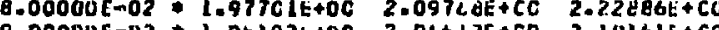

$9.00000 E-02 * 1.95103 t+00 \quad 2.06617 E+C O \quad 2.13141 E+C O$

$1.00000 E-01 * 1.92054 E+00 \quad 2.03634 t+C O \quad 2.15583 E+C U$

$1.10000 \mathrm{t}-01+1.90351 \mathrm{E}+00 \quad 2.00 B 15 E+C O \quad 2.122675+C 0$

$1.20000 E-01+1.28191 E+00$ 1.9815bE+CO 2.0DCLCE +CO

$1.30000 \mathrm{t}-01+1.86169 E+00 \quad 1.95652 E+C 0 \quad 2.05987 t+C O$

$1.40000 t-01 \cdot 1.84284 E+00 \quad 1.93362 E+C O \quad 2.03134 E+C O$

$1.50000 E-01+1.02532 E+00 \quad 2.921 L 1 E+C E \quad 2.0044 B T+C D$

$1.60000 t-01.1 .80909 E+00$ I. $09045 E+C O$ I. $97924 t+C 0$

$1.70000 E-01$ 1.704

1.80000E-01

1.0000E-OR 1.TBU37E+OO $1.8535 E E+C O \quad 1.93352 t+C O$

$1.90000 t-01 * 1.76782 E+O C \quad 2.83716 E+C O \quad 1.91253 E+C O$

$2.00000 E-03 \cdot 1.75644 E+00 \quad 2.822 C 7 E+C O \quad 1.89382 t+C O$

$2.10000 E-01$ 1.7461BE+0O $1 . H 0825 E+C O \quad 1 . B 7615 E+C O$

$2.20000 E-01$ 1.73703L+OO 1.792U8E+CO 1.85967E+CO

$2.30000 E-01$ 1.72894E+0O $1.78432 E+C O \quad 1.64494 E+C O$

$2.40000 E-01 * 1.72189 E+00$ 1.7.7413E+CO $1.83134 E+C O$

$2.50000 t-01 \cdot 1.71584 E+00$ 1.76507E+CO $1.81901 t+C O$

$2.60000 E-01+1.71077 E+00 \quad 1.75713 E+C O \quad 1 . B 0794 E+C O$

$2.70000 E-01 * 1.70665 E+00 \quad 1.75023 E+C 0 \quad 1.79806 E+C O$

$2.80000 E-01 * 1.70344 E+00$ L.74442E+CO $\quad 1.7893 \mathrm{Ht}+\mathrm{CO}$

2.900 UOE-OL $: 1.70112 E+00 \quad 1.73939 E+C O \quad 1.78180 E+C O$

$3.00000 E-01+1.69966 E+0 C \quad 1.73573 E+C O \quad 1.77533 t+C O$

$3.10000 E-01.1 .65403 E+00 \quad 1.73262 E+\varepsilon 0 \quad 1.76993 E+C O$

$3.20000 E-01 * 1.69919 E+00 \quad 1.7308 L E+C O \quad 1.76556 E+C O$

$3.30000 E-01 \div 1.70013 E+00 \quad 1.72969 E+C O \quad 1.76217 E+C O$

$3.40000 E-01 * 1.70182 F+00 \quad 1.72941 E+C O \quad 1.75975 E+C O$

$3.50000 \mathrm{E}-\mathrm{Cl} 1.704235+00$ 1.72995E+CO

$3.60000 E-01 * 1.70731 E+00$ 1.73128E+CO $1.75765 E+C O$ 


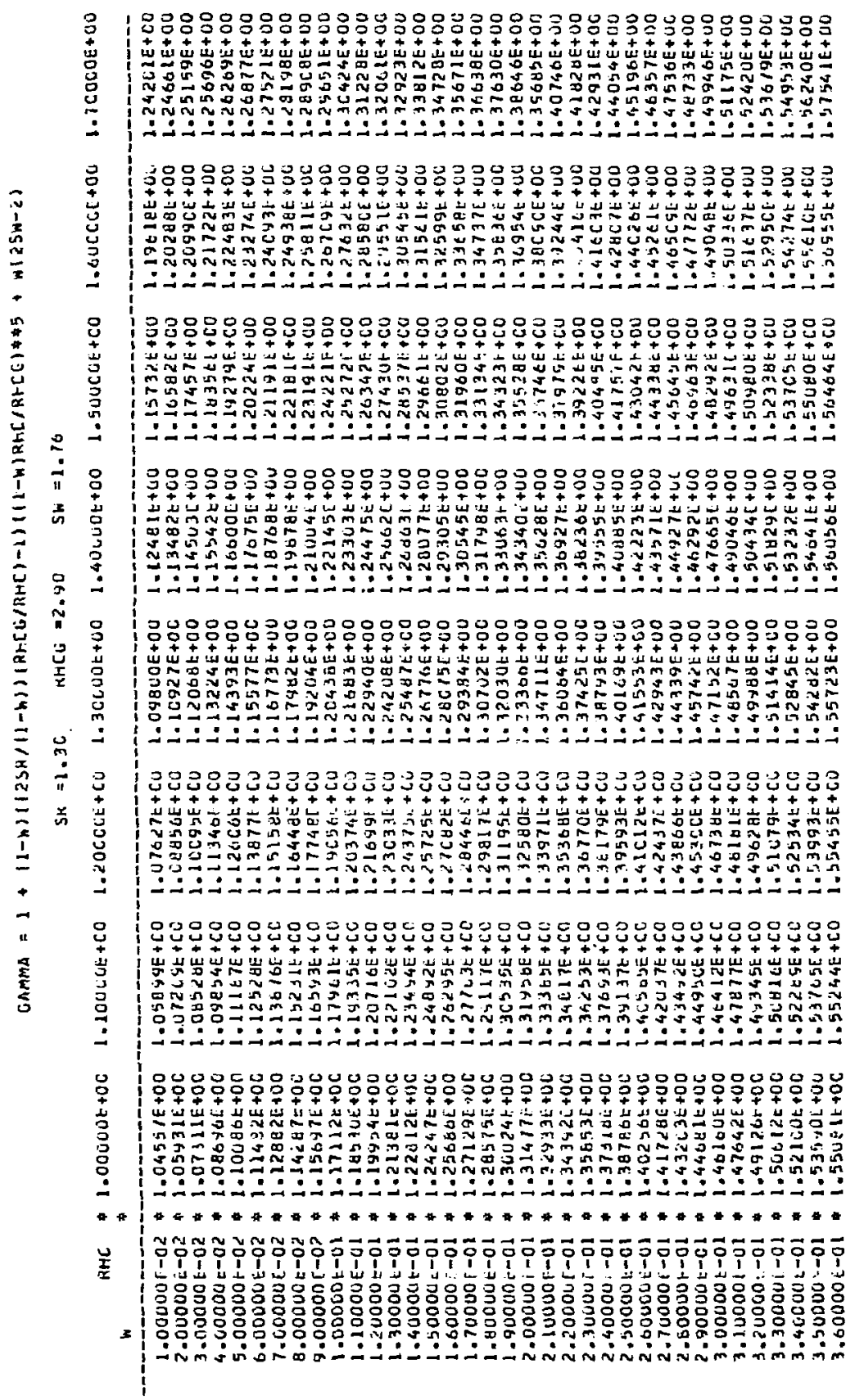




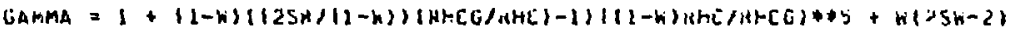

$S K=1.3 C \quad H H L C=2.90 \quad S h=1.7 t$ r

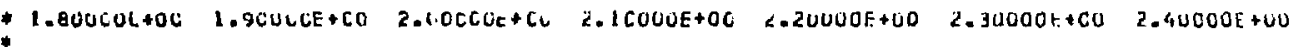

$.30000 E+00$

$1.00000 \mathrm{c}-02+1.29545 \mathrm{C}+0 \mathrm{C}$

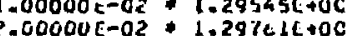
$3.00000 \mathrm{r}-02: 1.3 \mathrm{GU} 25 \mathrm{E}+0 \mathrm{C}$ $4.00000 r-0 .+1.303 j 4 E+00$

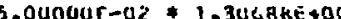
6.0000ur-0s 1.31GJtetoc -

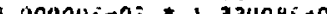
- 1.

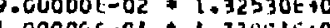
.0000sc-or 1.336sitet+oc - $100001=01$ 1.3364Ut +00 $1 . ? 0000 t-01+1.34327 \vec{E}+0$ $1.30000 t-E 1+1.34+59 E+0 C$ $1.600001-C 1+1.35707 E+00$ $-50000 r-01+1.36448 t+00$ $1.60000 k-01+1.37222 E+00$ $.70000 E-01 * 1.38029 E+00$

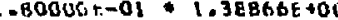
$.90000 i-01 * 1.39734 E 406$ $2.00000 i-01+1.406] 0[+0 c$ .100UOE-OL 1.43555t+oC $2 .<00001-01+1.425575+00$ . $300001-01+1.43445 t+00$ . $.40001-01+1.44489 E+0 C$ $.50000 t-01+1.43526 E+0$ . R0000r-01 * 1.46570E+00 $2.70000 \mathrm{r}-01+1.47646 \mathrm{E}+00$ 2.800001-01 1.48743E+0C $2.70000 \mathrm{t}-01+1.496 \mathrm{ezE}+0 \mathrm{C}$ $3.00000 \mathrm{~L}-01$ 1.51002E+00 $3.10000 E-01$. $1.52161 t+00$ $3.200001-01+1.533401+00$ $3.30000 E=01 * 1.54537 E+O$ $3.40000 E-01 * 1.53751 E+00$ $3.50000 \mathrm{r}-01+1.56923 E+00$ $1.34713 E+C O \quad 1.427 C B E+C U \quad 1.50762 C+00$ $1.33643 E+C 0 \quad 1.42671 E+C 0 \quad 1.493 b 7 t+00$ $1.35692 E+C 0 \quad 1.41826 t+C O \quad 1.487 \theta 7 E+00$ $1-35755 E+C C \quad 1.41847 E+C U$ 1. $48202 E+0$ $1.35 y+1 t+C C \quad 1.41523 E+C U$ 1.4784 $5 E+00$ $1.3+1,6 E+C O D 2.4146 C E+C D \quad 10474,14 E+C O$ $164146+C D$ I. $41465 t+C O$ $+C O \quad 1.41524 E+C O \quad 1.46930 E+60$ - 3crect $1.4164 \mathrm{Ct}+\mathrm{CU} \quad 1.46894 E+C U$ $1.4164 \mathrm{Ct}+\mathrm{CU}$ $1.46 \theta 94 E+C O$
$1.48740 E+00$ $.3791 C E+C O$ $1.42 C 34 t+C 0 \quad 1.46722 t+00$ $1.4231 C E+C E \quad 1.46734 E+C$ $1.324 c 32+c c$. $1.42637 t+C O \quad 1.46836 E+00$ $1.43 C 13 E+C O$ $1.47012 E+00$ .392 L $3 E+C L$ $1.40114 E+C C$ $1.407 C 4 E+C D$ $1.41131 E+C O$ $1.42175 t+C O$ $1.42933 t+C O$ $1.437<J E+C O$ $1.44,3 \rightarrow J E+C O$ $1.45411 F+C$ $1.454 J I F+C$ $1.4 E 3 L I E+C$ $1.47220 E+C O$ $1.43144 E+C D$ $1.47244 E+C 0$ 1. 5O148E+CO L. $51177 E+C O$ 1. $52231 E+C O$ $1.5331 \mathrm{AE}+\mathrm{C}$ $1.54412 E+C C$ 1. 55 ל $3 \triangle E+C O$ $t t+c 0$ $1.47224 t+0$ CtE+CO $1.47491 E+00$ $1.47 \mathrm{E} 12 \mathrm{E}+00$ $1.44982 E+C O$ $1.45583 E+C U$ $1.46226 E+C O$ $2.46908 t+C C$ $.48607 t+02$ 1.4907 $1.49547 t+00$ $1.48387 E+C 0$ $1.50161 E+00$
$1.50770 E+00$ $+c 0$ 1.51422 .. $51422 E+00$ tc $508725+c 0$ 5 $76 \%$ - 0 I. $52848 E+00$ $1.53619 E+06$ - S269 3e+co - $5050+c a$ $1.54635 E+C O$ (1) 1. $55273 \mathrm{E}+00$ 1. $56153 E+00$ 1. $57066 \mathrm{E}+00$ $1.5665 \mathrm{CE}+\mathrm{CO}$ 1. $58011 E+00$ $1.5664 \geq E+C O$ $1.57156 E+C O$ 1. $58987 \mathrm{E}+00$ $1.59158 t+00$
$1.58<22 t+00$ $1.57 \overline{79}=+00$ $1.56627 t+00$ 1.5574 $t=0$ 1. $54+85 E+40$ 1. $54+05 E+00$ $1.536810+00$ 1. 1. $520805+0$ $1.52319 r+00$ $1.52016 \mathrm{~L}+0$ L. $51786 t+0$ L. 5 Itis $5 t+00$ $1.51333 t+00$ $1.51507 \mathrm{t}+60$ 1.51 $346 E+$ UC 1.5L647f+U0 $1.51810 t+00$ $1.52331 E+C O$ $1.52310 t+00$ $1.52644 t+00$ $1.53032 t+00$ $1.53473 E+O Q$ 1. $33963 t+00$ . 54 bojetUd 1. SSUYOE+00 1. $55122 E+00$ 1. 57175 $1.57877 t+00$ $1.586766 E+00$ $1.54314 E+00$ $1.57848 E+C O \quad 1.58448 E+C O \quad 1.59993 E+00 \quad 1.61297 E+00$ $1.6102 \forall E+00 \quad 1.62240 t+00$

$1.444 C 6 E+C O$ $1.63760 t+c 0$ $1.05395 E+C U$ $1.64133 E+C O$ $1.624641+00$ 1. $619045+C 0$ $1.00933 \mathrm{l}+\mathrm{CC}$ $1.6005 j \mathrm{E}+\mathrm{CO}$ $1.5+2681+C 0$ $1.54564 \mathrm{E}+\mathrm{CO}$ $1.57955 t+\angle 0$ 1. $57420 F+C O$ $1.5 x+7 E E+C D$ 1. $56010 E+C 0$ $1.5631 \mathrm{BE}+\mathrm{CC}$ 1. $561 \mathrm{lCZ}+\mathrm{CO}$ 1. $5459 Y+00$ 1. $558 B 6 E+C O$ $1.55 B 83 E+C O$ 1. $55946 \mathrm{t}+\mathrm{CO}$ $1.50074 E+C O$ $562055+00$ $1.50510 \Gamma+C O$ $1.56 \forall 27 E+C 0$ $1.5119 E+C O$ - Srolite+co 1. $50093 E+00$ $1.59198 \varepsilon+C O$ $1.54 B 23 t+C O$ 1. $60494 E+00$ $1.61210 t+00$ $1.61969 E+C$ $1.62769 E+0$ $1.63609 E+00$
1. $069 D 6 E+U C$ $1.78895 E+0 U$ 1.7095 l $t+00$ $1.734355+00$ $1.718470+40$ 1. $71847 E+U D$ 1. $190 \mathrm{C} 45+00$ I. $61743 E+00$ $1.01587 t+u 0$ $1.055315+00$ $1.64575 E+U 0$ $1.63715 E+00$ $1.62949 t+00$ $\therefore .62274 E+U 2$ I. ClEEHE +UO 1. $611 \mathrm{BS}[+O \mathrm{O}$ $1.607755+00$ $1.00442 F+00$ $1.601885+00$ I-GUCLET+UO $1.395202+30$ $1.59202+50$ $1.59281 E+00$
$1.513923 E+00$ $1.51923 E+00$ $1.60033 E+00$ $1.60209 E+00$ . $60449 E+00$ $1.6075 C E+00$ L.ti 1.6IE $31 E+00$ $1.62 \mathrm{ccec}+00$ $1.62534 E+00$ $1.63115 E+00$ $1.63145 E+00$

$.64423 E+0 D$ 1. $5148 \mathrm{BE}+00$
$1.93253 \mathrm{E}+00$ 1. $9 \operatorname{Cos} 3 E+00$ $1.8 E 212 E+00$ 1. $858965+00$ $1.43313 E+00$ 1. $8160 U E+00$ $1.79734 E+00$ $1.779325+00$ 1. $102515+0$ 1. $74669 E+00$ 1. $73243 E+U 0$ I. $71909 E+00$ 1. $7 C G H E E+O O$ $1.64569 E+00$ I. 6 E55 日E + 00 $.6764 \mathrm{BE}+\mathrm{UC}$ $.66837 E+00$ $1.6 E 172 E+00$ $1.65502 E+00$ $.64972 E+00$ $1.64177 E+00$ $1.63905 E+00$ 1.63714E+00 $1.6002 E+00$

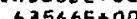
(5) ज . $6413 \mathrm{E}+00$ . $64133 E+00$ $1.64441 E+00$ $1.64811 E+00$ $1.65240 E+00$ $1.6572 \mathrm{BE}+00$ $1.66270 E+00$ $1.66867 E+00$ 
GAMMA $=1+(1-h)((2 S R /(1-h)(R H C G / R H[)-1)(11-h) R H C / R H C G) \neq \$ 5+h(25 h-2)$

$S H=1.30 \quad$ RHCG $=2.9 \mathrm{C} \quad S \mathrm{SH}=1.16$ H

RAC * 2.6000OE+DO 2.7000CE+CO 2.80CCOt+CO 2.90000E+00

$1.00000 E-02 * 2.06737 E+O C \quad 2.21443 E+C O \quad 2.374 C C E+C O 2.546241+00$

$2.00000 E-02 \div 2.03573 E+00 \quad 2.1700 U E+C O \quad 2.32953 E+C O \quad 2.49475 E+D O$

2.00565+00 2.1405LE+CO 2.2864OE+CU 2.49534E+OO

$3.00000-02+2.0056 E+00$ 2.140 $2.41534 E+00$

4.0

$3.00000 t-02+1.95047 .0 C 2.07342 E+C C 2.20757 E+C O 2.35274 E+00$

$6.00000-02$ 1.92444L.00 $2.04235 E+C 0$ 2.17C61E+CO $2.30948 E+0$

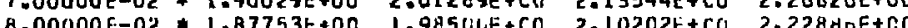

$8.00000 E-02 * 1 . B 7753 E+00$ 1.915ULE+CO 2.10202E+CO $2.228 \mathrm{CDE}+\mathrm{CO}$

$9.00000 \mathrm{t}-02 * 1 . B 5614 E+00$ 1.958hSF+CO 2.07C32E+CU 2.19141E+0O

$1.00000 E-01$ * 1.83010E+00 1.933EIE+CO 2.04CJUF+CU 2.1J583E+00

$1.10000 E-01 * 1.81736 \mathrm{E}+0 \mathrm{C} \quad 1.91043 \mathrm{E}+\mathrm{CO} \quad 2.01192 \mathrm{E}+\mathrm{CO} \quad 2.12267 \mathrm{E}+00$

$1.20000 E-01+1.79991 E+O C \quad 1.888 b C E+C C \quad 1.98514 t+C O \quad 2.09010 E+D O$

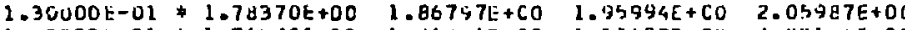

1. $.0000 t-01$ 1.766 $10 E+00 \quad 1.4460 L E+C O \quad 1.93627 E+C 0 \quad 2.03134 E+00$

$1.500001-01 * 1.75410 E+00 \quad 1.83048 E+C O \quad 1.91410 E+C O \quad 2.0044 B E+00$

$1.60000[-01 * 1.74225 E+O C \quad 1.81446 E+C O \quad 1.89339 t+C O \quad 1.97925 E+O C$

$1.700001-01+1.73072 t+00 \quad 1.799<2 E+C O \quad 1.8741 U E+C U \quad 1.45561 E+00$

$1.80000 \mathrm{~L}-01 \neq 1.720 .4[+00 \quad 1.785 \mathrm{ZLE}+C 0 \quad 1.4562 \mathrm{CL}+\mathrm{CO} \quad 1.93322 \mathrm{E}+00$

$1.90000 t-01$ 1.71U13t+00 $1.77240 E+C C$ 1.83964t+CO $1.5124 j E+00$

$2.000001-01+1.70241 E+00 \quad 1.70077 E+C O \quad 1.42444 E+C O 1.89302 t+00$

$2.10000 t-01+1.69524 E+001.750<B E+C 0 \quad 1.91 C 44 E+C 0 \quad 1.87615 t+00$

$2.100001-01$ 1.69529EtoD $1.750<8 E+C O$ I.

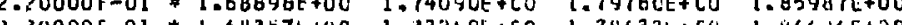

$2.30000[-01 \$ 1.68357 t+0 C \quad 1.1323 t+C C 1.78632 t+C O \quad 1.04434 E+00$

$2.40000[-01+1.67911 C+00$ 1.72s33E+Cn $1.77601 c+C 0 \quad 1.83134 t+00$

$2.50000+-01$ 1.67534E+00 1.71+Lyt+co $1.76685 t+C 0$ 1. $81901 E+00$

$2.60000 t-01$ 1.672E4t+0C $1.713 t 3 E+C 0 \quad 1.758 B U t+C U 1.907+45+00$

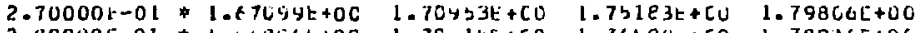

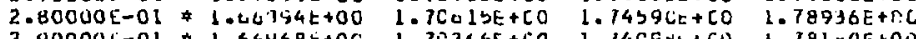

$2.90000 \mathrm{C}-01+1.66468 E+0 \mathrm{C}$ 1.703C6E+CO 1.14C5HL+CO 1.18100E+0O

$3.000005=01$ 1.6\%19E+0D $1.702 L 4 E+C 0$ 1.737C4E+CU $1.17533 E+00$

$3.10000 \mathrm{E}-01=1.67142 t+0 \mathrm{C} \quad 1.701<5 E+C 0 \quad 1.73404 t+C 0 \quad 1.76993 \mathrm{E}+\mathrm{CC}$

$3.20000[-01$ 1.67397E+0O 1.701<UE+CC $1.73196 E+C O \quad 1.76556 E+0 D$

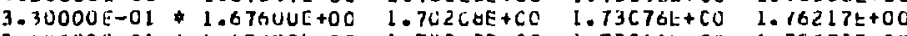

$3.40000 E-01=1.67430 t+0 C \quad 1.703 C 3 E+C O \quad 1.73 C 41 t+C 01.75975 E+00$

$3.50000[-01+1.68323 \mathrm{t}+0 \mathrm{C} \quad 1.705 \mathrm{yl}+\mathrm{CO} \quad 1.73 \mathrm{CBgE}+C 0 \quad 1.7582 \mathrm{EE}+00$

$3.60000 t-01+1.64777 t+0 C \quad 1.7 C E t 5 E+C O \quad 1.73215 t+C O \quad 1.75765 E+00$ 
BAHHA $=1+(1-h)((2 S R /(1-h))(R H C G / P H C)-1)(1) h)$ RHC/HACG)\$S+h(ZSW-F)

$$
S H=1.30 \quad \text { KHCG }=3.00 \quad S h=1.76
$$

RHC 1.0UUDOE+0O 1.10OUOE+CO $\quad 1.20000 E+C O \quad 1.30000 E+00 \quad 1.40000 E+00$

1.5OUCOE+CO 1.tOCCC $t+0 U$

1. $7 C C O U E+U O$

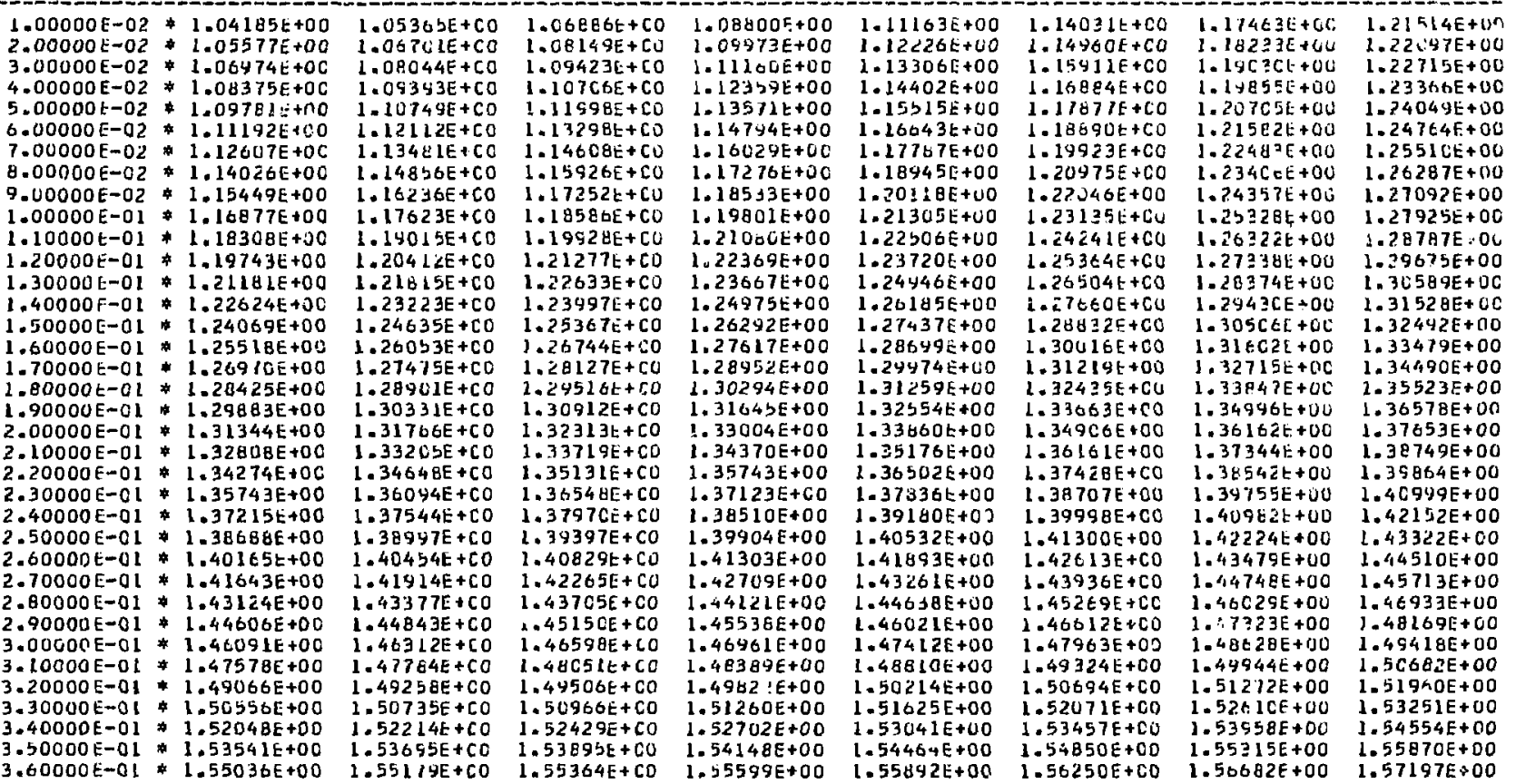




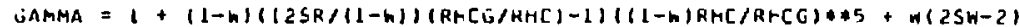

$S K=1.3 \mathrm{C} \quad \mathrm{KHCL}=3.00 \quad \mathrm{Sh}=1.76$

$1.00000 f-02 \cdot 1.26244 E+00$ $2.00000 t-02 * 1.26011 j k+00$ $3.00000 t-02 \$ 1.27019 t+0 C$ $4.000001-02 \times 1.274445 .00$ $5.00000 t-02+1.274$ ist +00 $0.00000 t-02 \$ 1.28485 E+0 C$ $7.00000 t-02 \cdot 1.256+1 t+0 c$ B. $00000 t-02 \div 1.29633 t+00$ 4.00OUUE-0. $\$ 1.302 \mathrm{i} C L+00$ $1 .(100041 i-0)+1.3 i 9 i s t+a C$ $1.100005-01+1.316721+00$ $1.200005-01+1.324114+00$ 1.20000 1.30000 $1.400005-01+1.33986 t+0$ $1.50000 t-01 * 1.34 \mathrm{~d} 18 \mathrm{~d}+00$ $1.80000:-01: 1.350 \mathrm{LUE}+00$ $1.70000 t-01 \& 1.36 b 71 t+00$ $1.400005-01+1.37408 t+00$ $1.50000[-01 * 1.38433 E+00$ $2.00000 \bar{t}-01 * 1.394 J 31+0 C$ $2.100001-01 * 1.403986+00$ $2.20000 t-01 \neq 1.41417[+0 C$ $2.300001-01 * 1.42460 \mathrm{E}+0 \mathrm{C}$ $2.40000 r-01 * 1.435<5 L+00$ $2.50000 t-01 * 1.44 t 12 E+0 C$ $2.6000 u t-02 * 1.45720 t+0 C$ $2.700001-01: 1.468496+00$ 2. $000005-01$ 1.47y47t+00 $2.80000 t-01$ 1.47997t+0C 3.9000 .000ut-01 $1.50348 E+00$ $3.10000 t-01+1.51251 \mathrm{tuc}$ $3.200001-01 \% 1.52770 t+0 c$

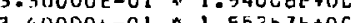
$3.40000 t-01 * 1.55257 t+0 C$
$3.50000 t-01 * 1.56523 t+0 C$ 3. $50000 t-01 \$ 1.56323 t+00$
$3.000001-01+1.578 C 4 L+0 C$

1. $317 \mathrm{CEE}+[\mathrm{CC}$ 1. $311+2 t E+C C$ 1. 31 YS $4 E+C C$ 1. $32 \angle 12 E+C O$ $1.32478 E+C O$ I. 32 B $\$ 1 E+C C$ $1.33145 t+C O$ 1. $.35 . \angle E+C O$ $1.33 \%, H E+C O$ 1. $3442 \mathrm{CE}+\mathrm{CC}$ $1.35 \cup 15 t+c 0$ 1. 3 . $5545+c 0$ 1.3SSE $4 E+C O$ 1. 36 E $M E+C C$ .37b1Gt+CO 1. Jo23 $35+C 0$ 1. 3P.यtE $+C C$ $1.3+1115+C 0$ $1.4 U 5 E B E+C O$ $1.4143 E E+C C$ $1.42313 F+C$ $1.44154 t+C 0$ $1.431<3 t+c 0$ $1.46113 \mathrm{E}+\mathrm{CO}$ $1.411<4 E+C C$ $1.4817 L E+C O$ $1.4817 C E+C O$ $\begin{aligned} & 1.44234 E+C O \\ & 1.5 C 3\end{aligned} 22 E+C O$ $1.51432 \mathrm{~F}+\mathrm{CO}$ 1. $525 \mathrm{LBE}+\mathrm{CO}$ 1. $53715 E+C O$ 1. $54 \varepsilon$ e $6 \mathrm{E}+\mathrm{CO}$ 1. 5 EC $70 E+60$ 1. $5 / \angle O D L+C O$
$1.5 E 511 E+C O$

\section{1. $37963 \mathrm{E}+\mathrm{Cu}$} 1. $3779 \mathrm{BE}+\mathrm{CL}$ 1. $37694 \mathrm{~L}+\mathrm{CL}$ UUE +C $1.37727 \mathrm{~L}+\mathrm{Cu}$ 1. $37845 t+\mathrm{co}$ 1. $10024 t+C 0$ 1. $3 \forall 251 \mathrm{k}+\mathrm{CL}$ $1.30524 t+C u$ $1.388445+C$ 1. $392245+C O$ $1.31648 \mathrm{~B}+\mathrm{Cu}$ I 1.40113 +ca $1.406212+C 0$ $1.4176 \% \mathrm{LL}+\mathrm{CL}$ 1.42397i + CC $1.4306 \mathrm{Bf}+\mathrm{CO}$ $1.437771+c i$ $1.44522 t+C i$ $1.453 \mathrm{C} 2 \mathrm{E}+\mathrm{LO}$ $1.46117 \mathrm{~L}+\mathrm{CU}$ $1.461,04 t+C 0$ $1.47844 k+C l$ $1.48754 \mathrm{t}+\mathrm{CU}$ $1.496945+C 0$ $1.49694 t+C 0$ $1.50663 t+c 0$ 1. $11059 t+c 0$ 1. $22 E E 2 E+C C$ $1.53731 L+C O$ $1.548 C 1 s t+c 0$ $1.555 \mathrm{C} 3 \mathrm{t}+\mathrm{Cu}$ $1.17 C 23 t+C u$ $1.561860+C 0$ $1.5133 \mathrm{Cf}+\mathrm{Cu}$

\section{$1.45063 t+00$} $1.4450 C E+D O$ $1.44165 E+00$ $1.4354 \times E+O$ $1.4934 \mathrm{Ct}+0 \mathrm{OC}$ $1.431+5 E+0 O$ $1.43113 E+00$ $1.43 U+2 E+00$ $1.43190 E+20$ $1.43220 t+20$ 1. $433(1) t+00$ $1.435 \mathrm{uat}+00$ 1.4384 PE+OC $1.4416 U E+U D$ $1.44313 t+00$ $1.44 \rightarrow, 5 E+U 0$ $1.4534=E+00$ $1.4590 \cup E+00$ $1.46450 t+00$ $1.47 U 43 E+O C$ i. $47679 \mathrm{E}+\mathrm{CO}$ $1.42354 E+00$ $1.49070 E+U O$ $1.4982 j E+U U$ 1. $500135+00$ $1.5141 d E+00$ 1. $522 \mathrm{VE}+0 \mathrm{O}$ $1.5314 u t+C O$ $1.54115 E+00$ 1. $55070 E+00$ $1.56 C D 5 E+00$ $1.57 C 085+00$ 1.5 B1 USE + 00 . $>9176 E+00$ 1. $6026 E[+00$

\begin{tabular}{|c|c|c|c|}
\hline $\begin{array}{l}+00 \\
+00 \\
+00 \\
+00 \\
+00 \\
+00 \\
+00 \\
+00 \\
00 \\
+00\end{array}$ & 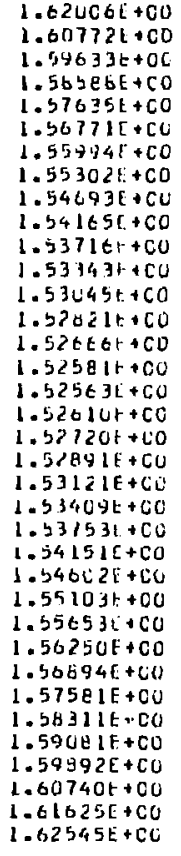 & 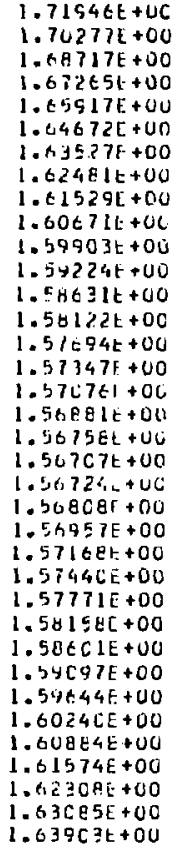 & $\begin{array}{l}1.82925 E+00 \\
1.8 C 779 E+00 \\
1.7 E 758 E+00 \\
1.76854 E+00 \\
1.75079 E+00 \\
1.73417 E+00 \\
1.11864 E+00 \\
1.70431 E+00 \\
1.69103 E+00 \\
1.67842 E+00 \\
1.66764 E+00 \\
1.65747 E+00 \\
1.64828 E+00 \\
1.64006 E+00 \\
1.63274 E+00 \\
1.62640 E+00 \\
1.62041 E+00 \\
1.61828 E+110 \\
1.61249 E+00 \\
1.6 C 9511+00 \\
1.6 C 733+110 \\
1.6 C 541 E+00 \\
1.6 C 523 E+00 \\
1.6 C 528 E+00 \\
1.66662 E+00 \\
1.6 C 744 E+00 \\
1.6 C 952 E+00 \\
1.61222 E+00 \\
1.61554 E+00 \\
1.61946 E+00 \\
1.62344 E+00 \\
1.62897 E+00 \\
1.63453 E+00 \\
1.64061 E+00 \\
1.04717 E+00 \\
1.65422 E+00\end{array}$ \\
\hline
\end{tabular}


GAMMA $=1+(1-k)((25 R)(1-k))(R H C G / A H C)-1)(1)-h) H H C / A L C G)+5+W(2 S W-2)$
$S R=1.30$
RHCG $=3.00$
$5 W=1.76$

KHC *

\begin{tabular}{|c|c|c|c|c|c|}
\hline $\begin{array}{l}.00000 \mathrm{E}-02 \\
.00000 \mathrm{E}-02 \\
.00000 \mathrm{E}-02 \\
.00000 \mathrm{E}-02 \\
.00000 \mathrm{E}-02 \\
.00000 \mathrm{E}-02 \\
.00000 \mathrm{E}-02 \\
.00000 \mathrm{E}-02 \\
.00000 \mathrm{E}-02 \\
.00000 \mathrm{E}-01 \\
10000 \mathrm{E}-01 \\
.20000 \mathrm{E}-01 \\
.30000 \mathrm{E}-01 \\
.40000 \mathrm{E}-01 \\
.50000 \mathrm{E}-01 \\
.60000 \mathrm{E}-01 \\
.70000 \mathrm{E}-01 \\
.80000 \mathrm{E}-01 \\
90000 \mathrm{E}-01 \\
.00000 \mathrm{E}-01 \\
10000 \mathrm{E}-01 \\
.20000 \mathrm{E}=01 \\
30000 \mathrm{E}-01 \\
40000 \mathrm{E}-01 \\
50000 \mathrm{E}-01 \\
60000 \mathrm{E}-01 \\
70000 \mathrm{E}-01 \\
80000 \mathrm{E}-01 \\
90000 \mathrm{E}-01 \\
00000 \mathrm{E}-01 \\
10000 \mathrm{E}-01 \\
20000 \mathrm{E}-01 \\
30000 \mathrm{E}-01 \\
40000 \mathrm{E}-01 \\
50000 \mathrm{E}-01 \\
60000 \mathrm{E}-01\end{array}$ & 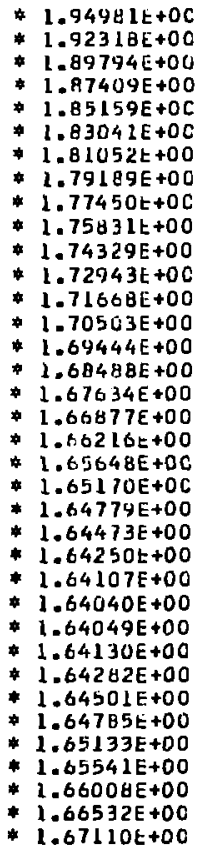 & 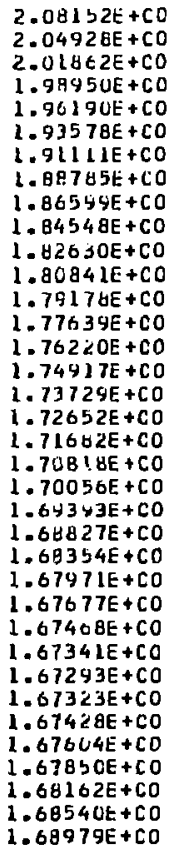 & $\begin{array}{l}2.2246 B E+C O \\
2.18641 E+C O \\
2.14951 E+C O \\
2.11512 E+C O \\
2.08202 E+C O \\
2.05 C 57 E+C O \\
2.02074 E+C O \\
1.99250+C O \\
1.96581 E+C U \\
1.94 C 63 E+C O \\
1.91693 E+C O \\
1.89469 E+C O \\
1.873 B E E+C O \\
1.85441 E+C O \\
1.83630 E+C O \\
1.81951 E+C D \\
1.40401 E+C O \\
1.78975 E+C O \\
1.77670 E+C O \\
1.764 B 4 E+C O \\
1.75413 E+C O \\
1.74453 E+C O \\
1.73603 E+C O \\
1.72857 E+C O \\
1.72214 E+C O \\
1.71670 E+C O \\
1.71223 E+C O \\
1.70 B 6 B E+C O \\
1.70604 E+C O \\
1.70427 E+C O \\
1.70335 E+C O \\
1.70323 E+C O \\
1.70391 E+C O \\
1.70534 E+C O \\
1.70751 E+C O \\
1.71038 E+C O\end{array}$ & $\begin{array}{l}2.37953 E+00 \\
2.33484 E+00 \\
2.2920 B E+00 \\
2.25122 E+00 \\
2.21223 E+00 \\
2.17507 E+00 \\
2.13970 E+00 \\
2.1 C 619 E+00 \\
2.07420 E+00 \\
2.04400 E+00 \\
2.01545 E+00 \\
1.98851 E+00 \\
1.96314 E+00 \\
1.93932 E+00 \\
1.91699 E+00 \\
1.89614 E+00 \\
1.87671 E+00 \\
1.8586 E+00 \\
1.84201 E+00 \\
1.82666 E+00 \\
1.81259 E+00 \\
1.79978 E+00 \\
1.78819 E+00 \\
1.77778 E+00 \\
1.76852 E+00 \\
1.16037 E+00 \\
1.75331 E+00 \\
1.74729 E+00 \\
1.74228 E+00 \\
1.73826 E+00 \\
1.73519 E+00 \\
1.73303 E+00 \\
1.73176 E+00 \\
1.73135 E+00 \\
1.73176 E+00 \\
1.73297 E+00\end{array}$ & $\begin{array}{l}2.54029 E+0 O \\
2.49475 E+0 D \\
2.44534 E+00 \\
2.39801 E+00 \\
2.35274 E+00 \\
2.30448 E+00 \\
1.26120 E+00 \\
2.22986 E+00 \\
1.19141 E+00 \\
2.15138 E+00 \\
1.12207 E+00 \\
1.09010 E+00 \\
2.05967 E+00 \\
2.03134 E+00 \\
2.0044 B E+00 \\
1.97925 E+00 \\
1.9551 E+00 \\
1.93152 E+00 \\
1.91293 E+00 \\
1.89382 E+00 \\
1.87615 E+00 \\
1.85987 E+00 \\
1.84444 E+00 \\
1.83134 E+00 \\
1.82911 E+00 \\
1.80794 E+00 \\
1.74806 E+00 \\
1.78936 E+00 \\
1.78180 E+00 \\
1.71533 E+00 \\
1.76993 E+00 \\
1.76556 E+00 \\
1.76217 E+00 \\
1.75975 E+00 \\
1.75826 E+00 \\
1.75765 E+00\end{array}$ \\
\hline
\end{tabular}


Table B3. Values of $\gamma$ for various combinations of weight fraction of water (W) and total porosity ( $\phi \equiv P$ HI). These values would be used when $S_{B}>130 \mathrm{kbar}$.

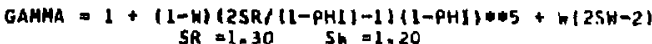

\begin{tabular}{|c|c|c|c|c|c|c|c|c|}
\hline 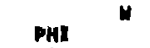 & 0. & -02 & -02 & $=-02$ & 1.0 & .0 & $\mathrm{E}=\mathrm{O}_{2}$ & $5-$ \\
\hline $\begin{array}{l}1000 \mathrm{E}-02 \\
000 \mathrm{E}-02 \\
0000 \mathrm{E}-02 \\
0000 \mathrm{E}-02 \\
1000 \mathrm{E}-02 \\
0000 \mathrm{E}-02\end{array}$ & $\begin{array}{l}28 E+00 \\
19 E+00 \\
394 E+00 \\
52 E+00 \\
392 E+00 \\
111 E+00 \\
109 E+00 \\
183 E+00 \\
33 E+00 \\
157 E+00 \\
53 E+00 \\
20 E+00 \\
56 E+00 \\
59 E+00 \\
29 E+00 \\
162 E+00 \\
59 E+00 \\
16 E+00 \\
33 E+00 \\
00 E+00 \\
39 E+00\end{array}$ & 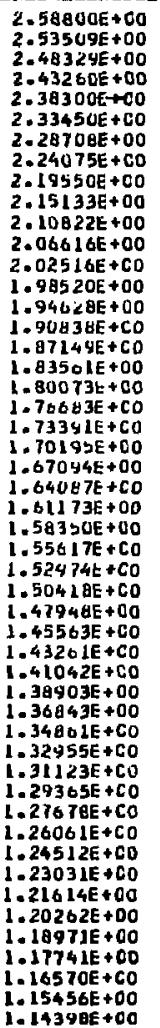 & 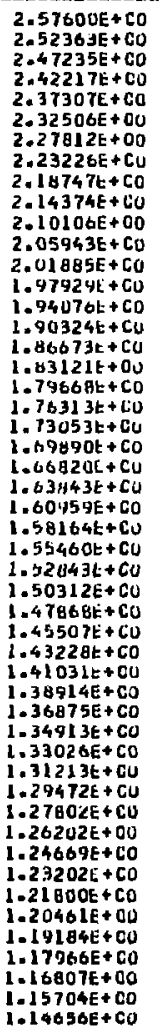 & 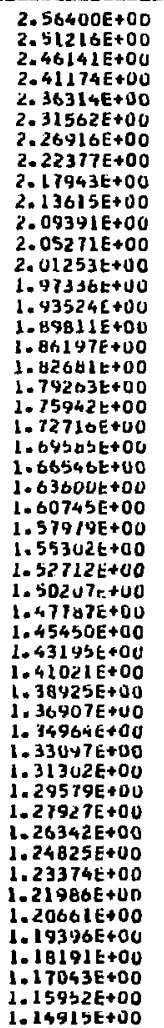 & 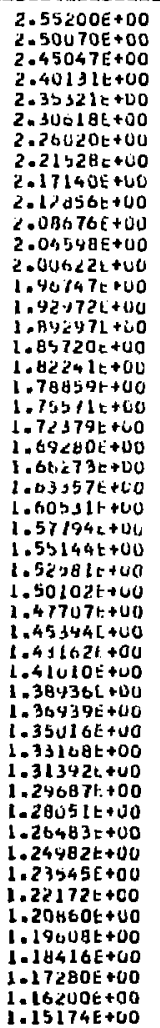 & 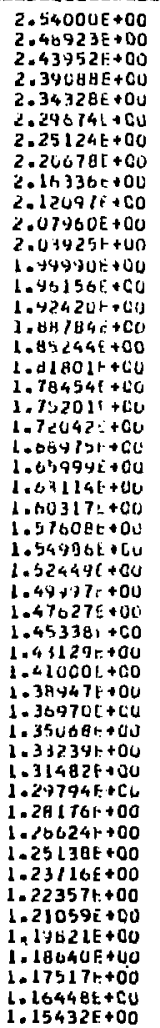 & 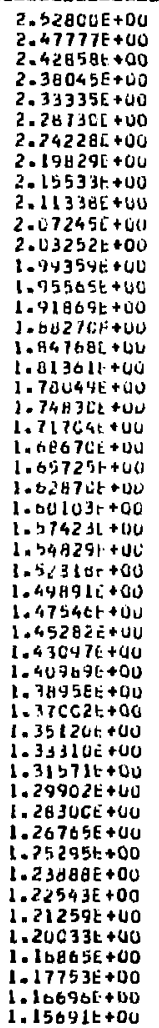 & 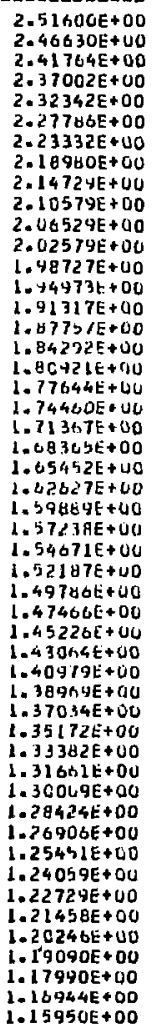 \\
\hline
\end{tabular}

S.00000E-0I $1=14139 E+00$

1.1362E+CU 1.14915E+00 $1.15174 E+00$

$1.144645+00$ 1.15691E+DO $1.15950 E+00$ 


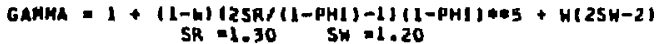

W. 6.00000E-02 9.00000E-02 1.00000E-C1 1.10020E-01 1.20000E-01 1.30000E-01 1.40000E-01 1.50000E-01 mit

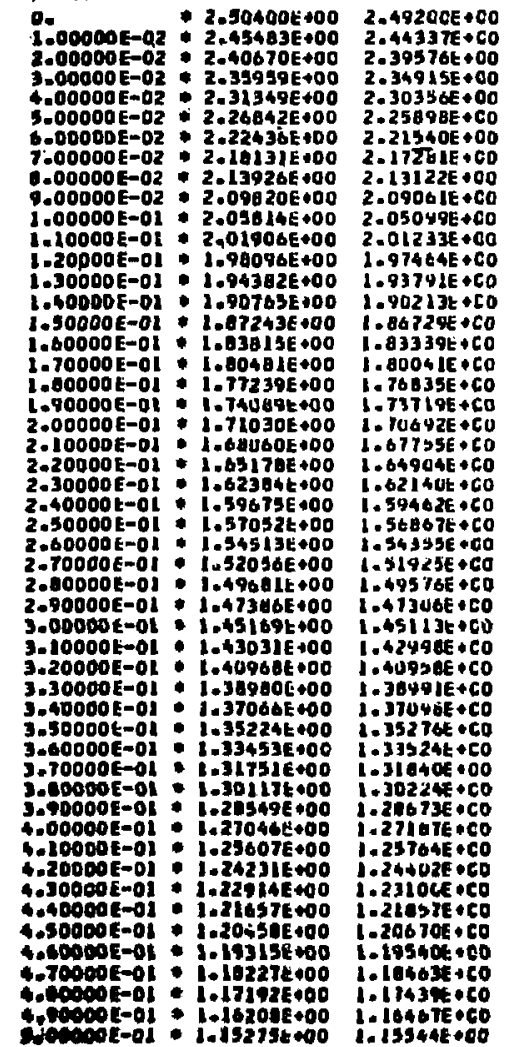

2.48000E+CO $2.43190 E+C 0$ $2.38481 E+C 0$
$2.33872 E+C O$ $2.34366 E+C O$ $2.24954 E+C O$ $2.20644 E+C U$ $2.12319 t+00$ 2.0B30.AE +CU 2.043BAE $+C U$ $2.00500 k+C 0$ $1.941833 E+C U$ $1.93200 z+c u$ l.B96bletco 1.Ac,21at+cu i. $6 r .653 t+c 0$ - r 1.7374at $1.70355 E+C U$ $1.07450 E+C O$ I.64631t+CJ 1.41 1897+co $1.59244 E+C$ I.5A17AE -CU I.SI TYAE. CO l.474225t+Eu 1.43CSFte 50 $1.420655+C 0$ $1.34002 t+C O$ I. $37130 t+C u$ i.3912at o 1.3jsgstec 1. $\$ 1430 t+c 0$ $1.207960+c 0$ $1.2732 u k+C u$ $1.25920 E+C$ 1.24573t:00 I.232ast *cu i.20eeje+co 1. $14765 \mathrm{~L}+\mathrm{CD}$ 1.jerces 00 I.176)e +co 1.jotzst + 100
2.46800E $\div 00 \quad 2.45600 E+00$ 2.42044E $\$ 00$ 3.40447E 00 2.3 $3367 E+00 \quad 2.36243 E+00$ 2. $32829 E+00$ 2. $24010 \mathrm{E} 00$ at $+00 \quad 2.18832 t+00$ $2.15543 t 00$ 2.14 1346000 2.11515E+00

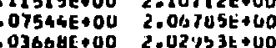
. $2.02 \% 33 \mathrm{k}+00$ 1. $81201 \mathrm{t}$ 1.92609e+00 1. I. H?JU7E+OU 791 CIE $\$ 00$ 1.729705000 i. rouI UE +OC 1.671456000 1.6435 te+U0 1.G16j4t+00 $1.596+46+40$ isquateod - 516636000 1.493635000 i. $430016+00$ $1-42 \times 12 E+00$ 1.409J0E400 iriczte00 i. is isutoos 1. 33606ENOU $1.3049 k+04$ $1.24922 E+u v$ 1.27469terd l. $24745+00$ i.214rzenuy 1.222565000 1.21045tiog 1. 364uncedo 1.189376000

1.i7y3\$t:00 1.169446000
1.100016000
$2.44400 E+100$ 2.351995 000 2. 3137431000 $2.22122 t+00$ $2-174501: 00$ $2.09408 t+00$ ä-citozort:00 $2.07237 t+00$ 1.3BSHJE +0 $1.44438\left[\begin{array}{cc}0 \\ 1.41437100\end{array}\right.$ 1.8HUUbe+OO $1.84075 t+c 0$ I. HIA34CiC l. $152160+C 0$ i. $132372+00$ $1.64143 t+C 0$ i. $1.11011+c 0$ I. gliteoscou $1.5 h 12 b t+c 0$
$1.33724 x+c 0$ l.33724e1co l. $111350=10$ 1.46404100 $1.43068+\% C 0$ i.4rabst+co I. 1.1136 \& 0 i. $372261+00$ 1. S54EAE ocu 1. 3uncest 400 1. $3>19 \% 50$ 1. 3065tE 00 i.dilizt $* C$ 1-2us9ol oco i.dustectes I.22654t 00 I. $215200 \cdot c 0$ 1.20434t 400 1. 194luenuo $1.144316+00$

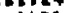
i:mative
$2.43200 E+00 \quad 2.42000 E+00$ $2.38604 E+00$ 2.37458E+00 $2.33010 E+00$ 2.25 2.7117UE 2.16104E+UD $2.13035 \mathrm{E}+00$ 2. $21 \mathrm{BSE}+00$ $2.041045+002.083016+00$ $2.05267 t+00$ 2.0450BE०णO $2 . M I S 22 t+00 \quad 2.00806 E+00$ $1.479645+00 \quad 1.47196 E+00$ $1.443 \mathrm{C} 7 \mathrm{C}+00$ 1.93613E 60 1. FOA J6E 1.drasale +00 1.86903E+0O 1.8364RE+0O

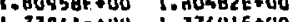
L.148IIE tuU l.

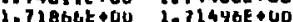
1.69OCGL \&UU I.6BGL9E+UU $1.6 n 2$ litelUO I. $55425 E+00$ I.t3b3ta+ou l.63262E+40

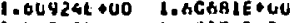
$1.3+3926+00$ L.3817at+ou i.jigacruo insShbe+00

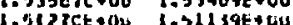

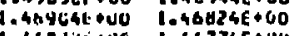

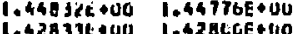

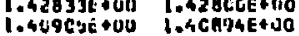

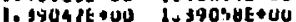
1.37257L+00 l.37289t०00

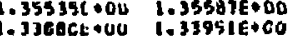

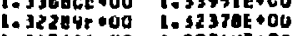
1. 307615.00 1.30464E.00 $1.7925 t+00$ 1.29420E•00

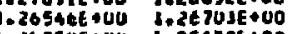
$1-2325 v E 000$ 1.25430E $\div 00$ 1.220541 .00 1.230\}JE⿰00 1.2171JE 000 l.21945t:00

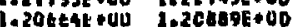
1. 14647E +UD 1.1980IE +00

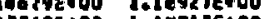
l.t7mecr 


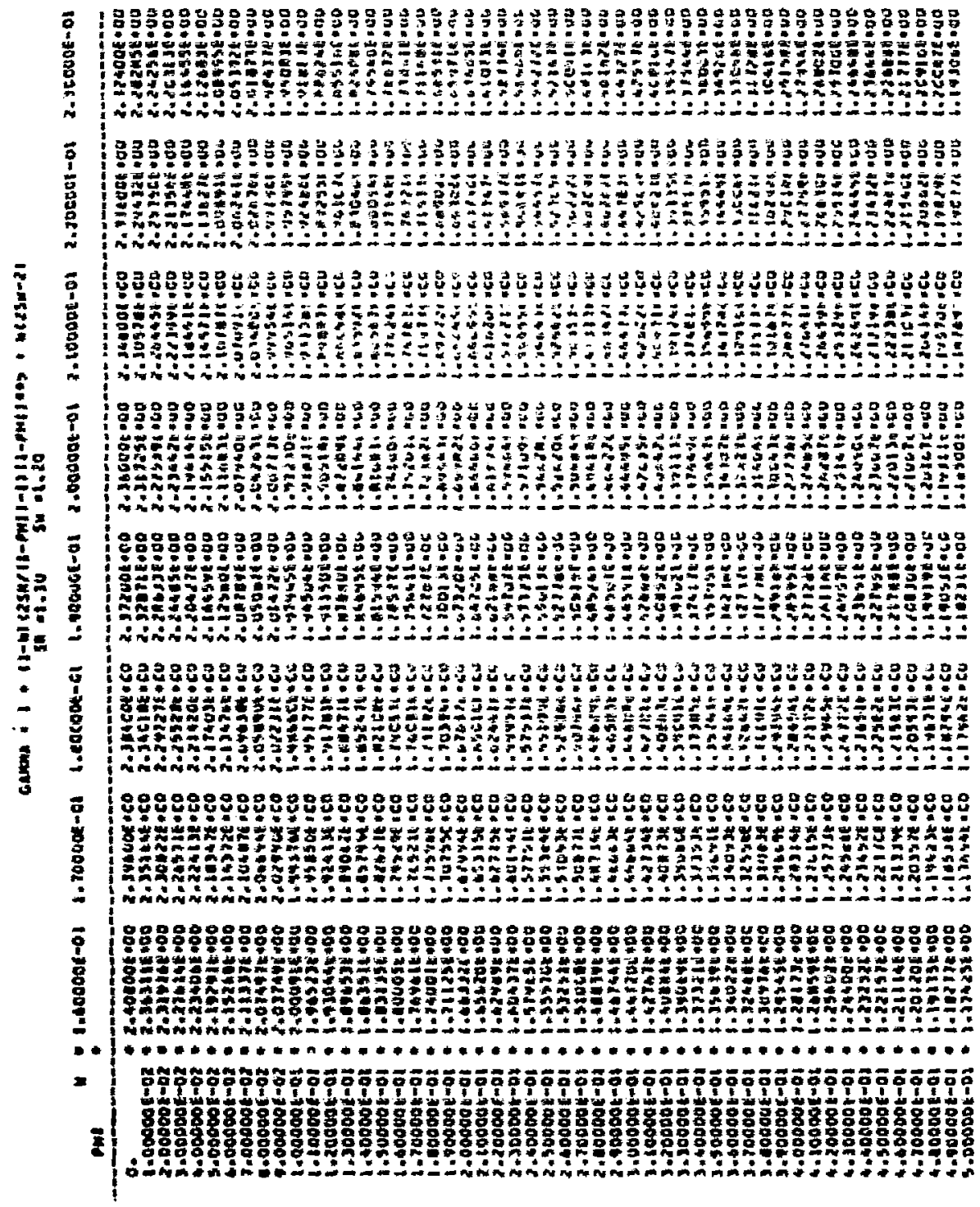




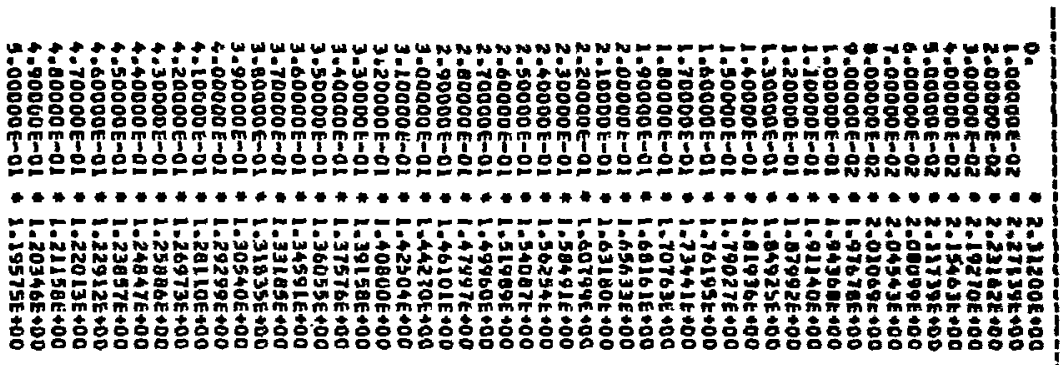

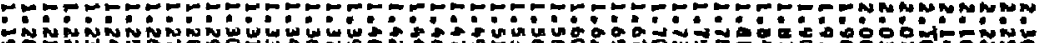

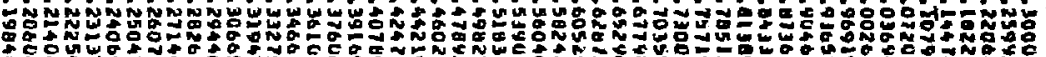

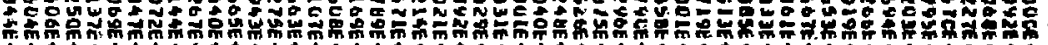

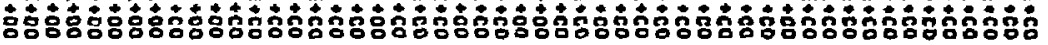

oñ

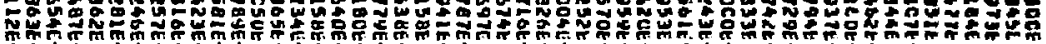

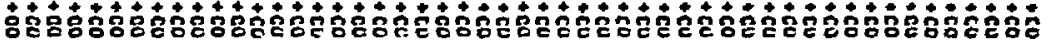

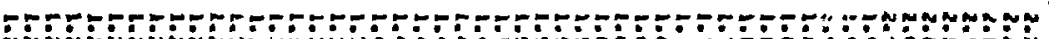
ONNNNSNFN

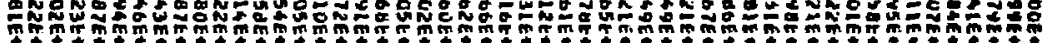

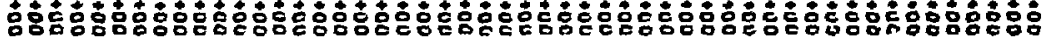

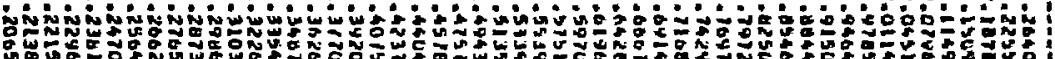

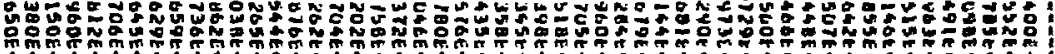

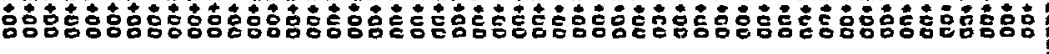
oñ

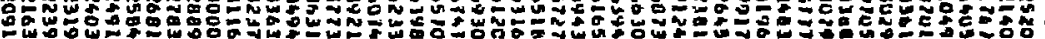

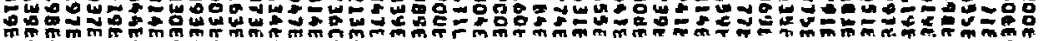

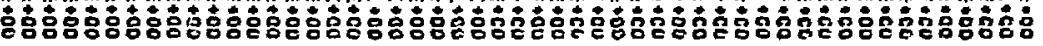

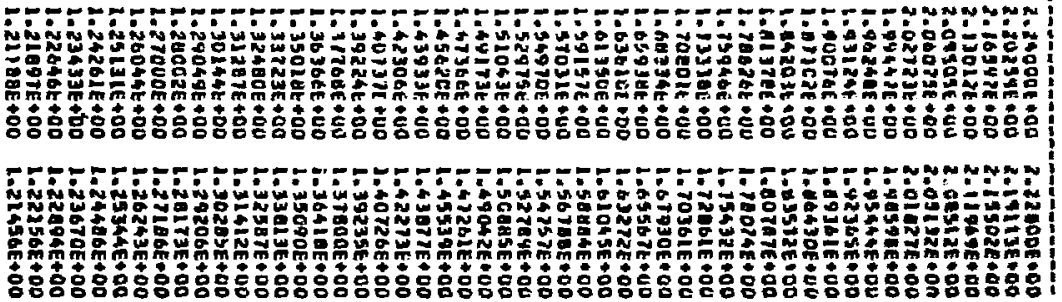


GAMHA $=1+(1-W)(25 K /(1-P H I)-1)(1-P H I)+45+W(25 W-2)$

$5 A=1.30 \quad 5 W=1.20$

W.200006-OL 3.30000E-Cl 3.40000E-CL 3.50000E-OL

\begin{tabular}{|c|c|c|c|c|}
\hline 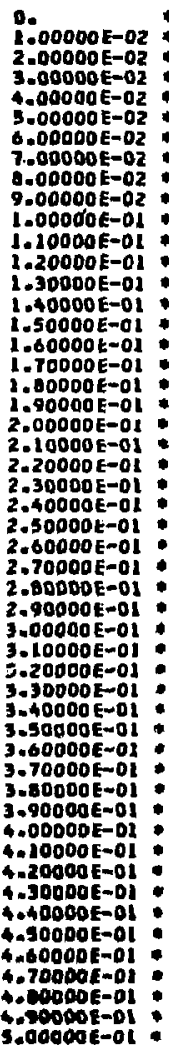 & 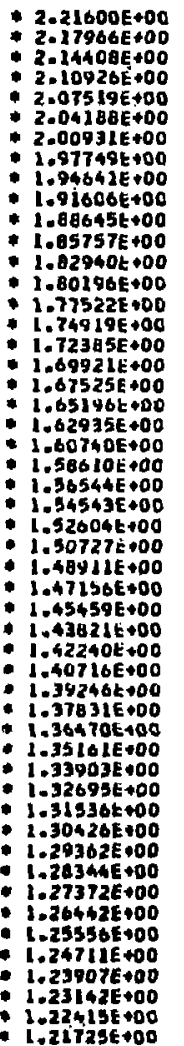 & 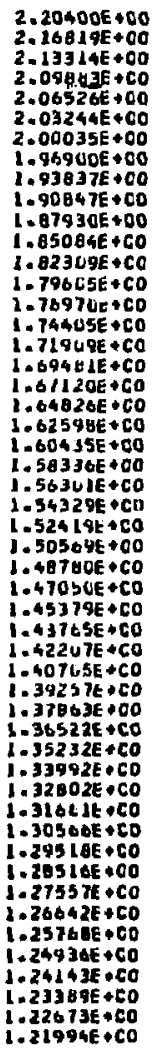 & 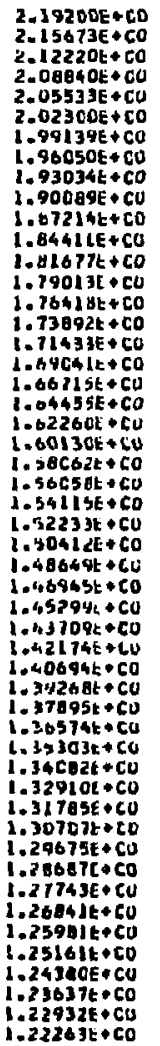 & 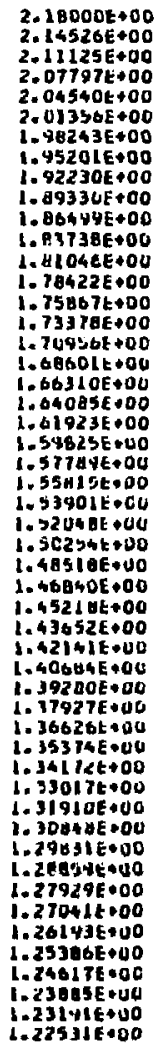 \\
\hline
\end{tabular}


Table B4. Values of $\gamma$ for various combinations of weight fraction of waler (W) and tolal porosity (4 $=$ pif). These values (rould be used when $\theta_{a}=130 \mathrm{kbar}$.

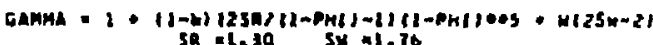

$$
50=10.10 \text { 5x } 41.70
$$

PHI

- 0.

1.00000E-C2 2.00000E-C2

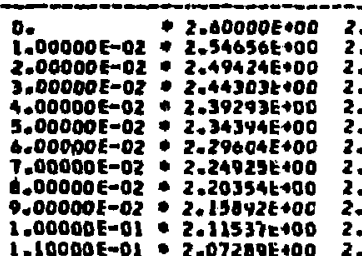

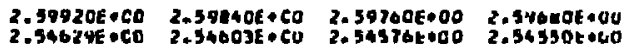

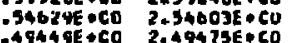
2. S4s76t:00 $2.44514 E O U C$
$2.396746+00$ $4438 \mathrm{BE}+C O \quad 2.44457 t+C$ $2.34570 E+C O \quad 2.34746 E+C O$ $3.20420 E+C O$ Z.30OS2E.CO $2-25$ (4t) +5 $2.2 C b 70 E+C 0$ 2.16253E 1190 $2.07736 E+C 0$ 2.01ESAE $* C 0$ 1.9964DE OCO $1.95746 E+C$ I. $914 B 8 E+C O$
I. 1.846bIE OCA 1.8H Y3E +Ca I-TASIIE +CO 1.T13LEE $+C$ $1.69207 E+C 0$ $1.62243 t+C O$
$1.5967 \mathrm{CE}+C D$ $1.56737 \mathrm{t}+\mathrm{CO}$ $1.54094 E+C D$
$1.51538 E+C O$ $1.51538 E+C O$
$1.4406 B E+C O$ 2.3546BE+CO 2525000 $2.20981 t+C 0$ $2.12346 E+C O$ $2.00103 E+C$ 2. $04125 t+C U$ $2.00169 t+C U$ l- $-425646+C 0$ $1.80413 t+C$
$1 .+553 t$ I. H53ELt +CO
I. $B 1$. $C A E+C O$

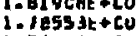
1. $75291 \mathrm{t}+\mathrm{CO}$

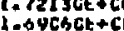
$1.3 B C 93 t+C 0$ 1. $031491+C$ $1.5770 u t+C$ $1 . S \rightarrow C H 3 E+C O$ $1.167552 t+c 0$
$1.201 C B E+C O$ $1.47747 t+C O$ $1.4436<E+C O$ $1.454 d 8 t+C O$ $1.41154 E+C U$ $1.39114 t+C y$ $1.40023 E+C 0$ $1-35941 E+00$ $1.34075 E+C 0$
$1.32243 E+C 0$ $1.32243 E+C 0$
$1.30485 E+00$ $1.2879 A E+C D$ $1.271 B 1 E+C D$ $1.35266 \mathrm{C}+\mathrm{CL}$ $1.33453 E+C O$ -31712E +Cu
I. 3OC4EE +CU $1.28442 E+C 0$ $1.28909 t+C$ $1.25442 E+C O$ $1.22702 \mathrm{E}+\mathrm{CD}$ l. $21424 t+c 0$ $1.20206 \mathrm{t}+\mathrm{CO}$ $1.19047 E+00$
$1.17944 t+C O$ $1.16096 E+C 0$
$1.13902 t+c 0$

\begin{tabular}{|c|c|c|c|}
\hline $\begin{array}{c}00 \\
00 \\
00 \\
00 \\
00 \\
00 \\
00 \\
0 \\
0 \\
0 \\
0 \\
0 \\
0 \\
0 \\
0 \\
0\end{array}$ & 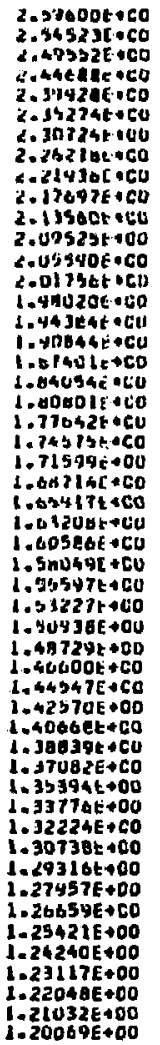 & $\begin{array}{l}00 \\
00 \\
0 u \\
00 \\
00 \\
00 \\
00 \\
00 \\
00 \\
00 \\
00 \\
00 \\
00 \\
0 \\
00 \\
00 \\
00 \\
00\end{array}$ & $\begin{array}{l}\text { i. } \\
\text { i. } \\
\text { i. } \\
\text { i. } \\
\text { i. }\end{array}$ \\
\hline
\end{tabular}




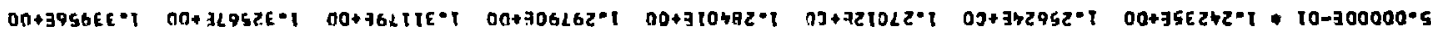

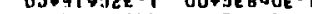

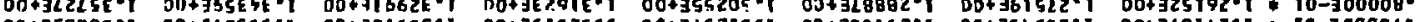

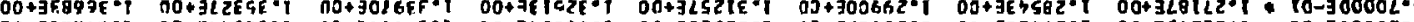

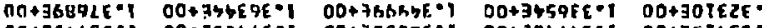

$00+355,0$

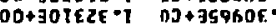

$03+7 E 802 E^{-1}$

nO+ ThE 5 ut :

nor

$00+39$ SZEF:

$0 J+305 \angle 0 E \cdot$

$00+35 \angle 282 \cdot 1+10-300009-$

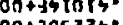

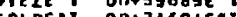

Dot3cis SE:

$03+3 \ell E 6 T E:-1$

$05+7 F L 1 G E:$
$03+30212 E:$

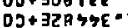

$0+3 \angle 1908+1 \div 10-300007-$

$00+36 n 562$

$00+192224 \cdot$

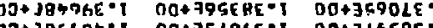

$0,3 \rightarrow 4856-1$

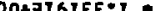

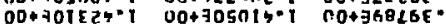

$05+7 B 254 E-$
$02+78 B B 6 E$ -

$00+3 \ell \neq ?: 16:$

$30+79 \ell 645=$

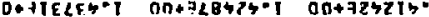

$03+3 T 6.57 \div-$

$00+315<05=$

$00+7578<6=1$

$00+798645 \cdot 100+76<124 \cdot$

09+70E165-

- 10-300001-4

$00+390098=1,10-900000^{-4}$

$00+7605$ LE- $10-300006^{\circ}$

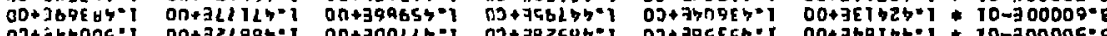

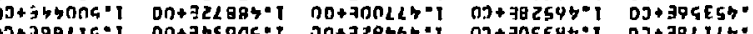

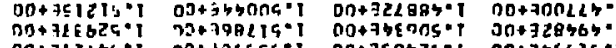

$00+3685>5 \cdot$

$0 n+385865:$

$00+19655 s^{\circ}$

$03+30 \varepsilon \varepsilon \theta^{2}$

$03.3811<2$.

004368156.1 10-300005.

. 10-30000क.

$00.704626-1 \cdot 10-30000 E$

作

$00+350105 \cdot 1$

$03+38 / 0,5=1$

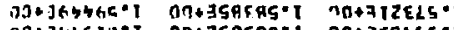

$0 n+392517 \cdot 1 \quad 00+721500 *$

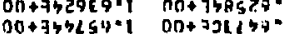

$00+3656<1901$

$0 n+005644=$

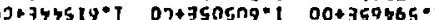

$00+31505 \cdot$

$03+386155 \cdot$

$00+3156 \mathrm{rs}-10 \mathrm{~T}$

$00+305145.1000000$

$00+394895 \cdot 1$ : 10-300005-

(10-700008*Z

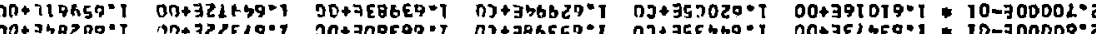

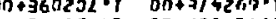

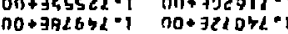

an+3ites

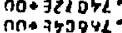

$00+370050+1$

$00+391 \geq 62:$

$90+32<5<9 \cdot 1$

$03+392020 \cdot$

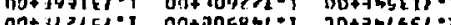

$(13+7288<9 \cdot 1$

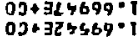

$00+321099 \cdot 1 * 10-300005 \cdot 2$

$00+395989 \cdot 10-300004^{\circ}$

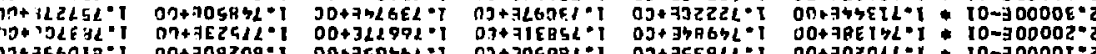

30156017 0 +

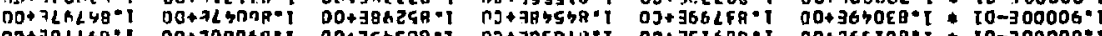

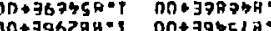

$00+390216 \cdot 200+715436=1$

$00+3102 \rightarrow A \cdot 00+712566^{\circ}$

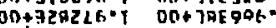

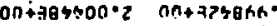

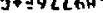

$00+364088^{-1}+10-300006$

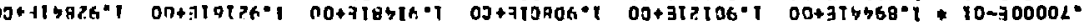

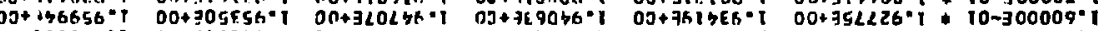

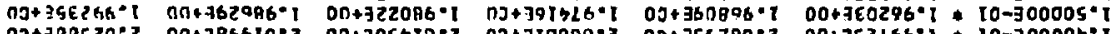

$00+3540 \angle 0^{\circ} 200+791590^{\circ}-2 \quad 00+720650^{\circ}$

$00+35140 T^{\circ} 5 \quad 00+7 \angle R 660^{\circ} 5 \quad 03+7854610.8$

$00+3966 E 1.2 \quad 30+3545 E t$

$001390117200+720211$.

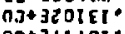

$00+728191 \cdot 2$
$00+39851120$

$00+786610^{\circ} \cdot 8$

$0,00+70, \rightarrow 10.5$

$03+3 E B C D O-2$
$02+3128 C 0-2$

$00+352166-1+10-300006$.

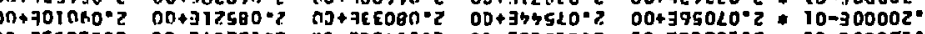

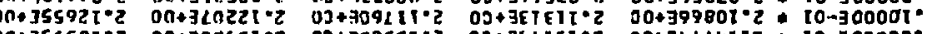

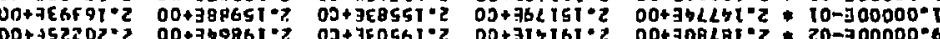

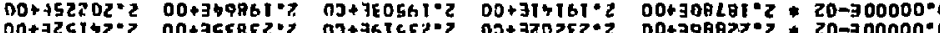

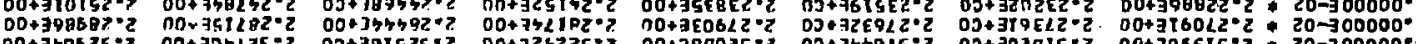

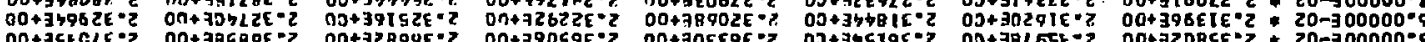

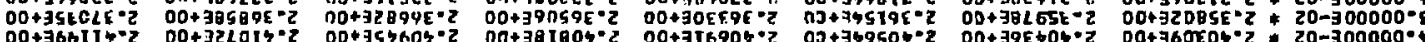

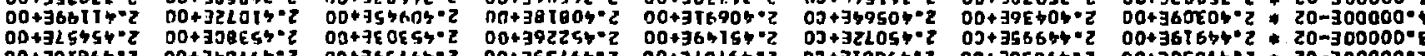

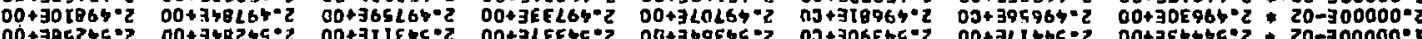

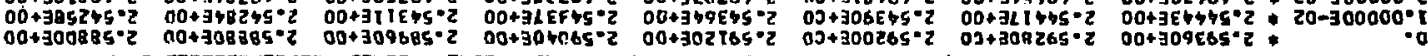

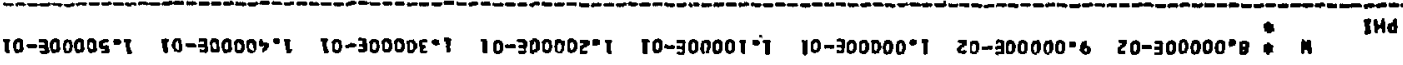

25.I= MS OE. I= 45

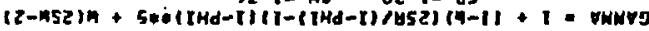


GAMHA $=1+(1-W(2 S R)(1-P H 1)-1)(1-P H) \cdots+5+W(25 w-2)$

PHI

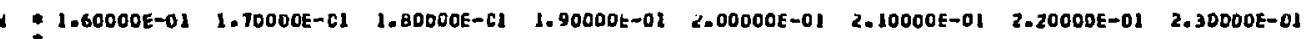

$2.00000 \mathrm{E}-02: 2.49836 \mathrm{E}+00$

T.00000E-02:2.45534E+00

5.00000 E-02 *2.37211E+00

$6.00000 E-02 * 2.33188 E 400$

$.00000 \mathrm{E}-02: 2.25417 \mathrm{2}+00$

$9.00000 \mathrm{E}-02+2.21669 \mathrm{E}+00$

$1.10000 E-01 \cdot 2.14443 E+00$

$1.20000 E-01$ ₹.10964E+00

$1.40000 \mathrm{E}-01: 2.04271 \mathrm{E}+00$

.50000E-01 $2.01055 \mathrm{E}+00$

$1.60000 \mathrm{E}-02: 1.91925 \mathrm{E} 400$

1.80000E-01: 1.91921E400

$1.90000 \mathrm{E}-0 \mathrm{~L}: 1.89045 \mathrm{EE}+00$

$2.00000 \mathrm{E}-01$ - 1. 1.46252E+00

$2.10000 E-01: 1.83540 t+00$

$2.20000 \mathrm{E}-0 \mathrm{~L}+1.80909 \mathrm{E}+00$

$2.30000 E-41: 1.78357 E+00$
$2.40000 E-01$
$1.758 d S E+00$

$2.50000 \mathrm{E}-01 \cdot 1.73490 \mathrm{E}+00$

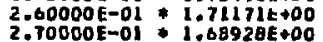

$2.70000 E-01 * 1.68928 E+00$
$2.00000 E-01 * 1.66759 E+00$

$2.90000 \mathrm{E}-01$ 1.04664Et00

3.00000 E-01 $1.82640 E+00$

$3.10000 \mathrm{E}-01: 1.80687 \mathrm{E}+00$

$3.30000 E-01 * 1.56989 E+00$

$3,40000 E-C 1: 1.55241 E+0 D$

$3.50000 \mathrm{E}-01+1.53559 \mathrm{E}+00$
$3.60000 \mathrm{E}-01 * 1.51942 \mathrm{E}+00$

$3.70000 \mathrm{E}-01$ 1.50380E+0O

$3.80000 E-01$ 1.48846E+00

$4.00000 \mathrm{E}-0 \mathrm{O}$. 1.46093E+00

$4=10000$ E-01 i.447TaE40

$4-20000 E-01 * 1.43522 E+00$

$4.30000 E-01+1.42320 E+00$

$4.40000 k-01+1.41172 t+00$
$4.50000 t-01+1.40077 t+00$

$4.60000 E=01$ 1.39034E40

$4.70000 E-01: 1.38040 E+00$

$4.90000 E-01$ 1.36197E 100

4.90000E-01 1.36197E $\$ 00$
$5.00000 E-01$
$1.35345 E+00$

$2.58640 E+c 0$

$2.54204 E+C O$
$2.49862 E+C O$
$2.4562 .1 E+C O$

$2.54178 E+C U$
$2.49887 t+C D$

$2.5848 D E+00$
$2.54151 E+00$

$2.37347 E+C O$

$2.45765 E+00$

$2.49439 E+00$

$2.58320 E+00$
$2.54098 E+00$
$0.44465+00$

$2.45919 E+00$

$2.38091 t+00$

2.34307E+00

$2.30064 E+40$ 2a $303405+00$

2.26050500 (2)

$2.22391 t+C O$

$2.2 \angle Q 2 D E+C D$

$2.20643 E+00$

$2.23113 E+00$

$2.19630 t+00$

$2.23474 E+C O$

$2.20034 t+C O$

-1
-1

.11453E+Ca $2.11941 k+C$

$1.492131+C 0$

$.98569 E+C D$

$.92636 \mathrm{~F}+\mathrm{CO}$

$1=80544 t+C J$

- $84335 E+60$

$.79234 E+C O$

1.76741E+10

$.72424 E+C O$
$.72133 E+C D$

$.69917 E+C 0$

$43 E+C 0 \quad 1.60743 F+C U$

(1)

$.61774 E+C O$

$.58120 E+C O$

$\begin{array}{ll}.51731 E+C O & 1.55903 E+C U \\ .53133 E+C D & 1.5+324 t+C O\end{array}$

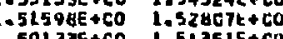

- $50123 E+C O$

1.

$46055 E+00$

$.44813 E+C D$
$-43626 E+C O$

$1.42492 E+00$

$1.40379 E+C O$

$1.39397 E+00$

$.37576 E+00$
1.3757
$2.08631 E+C O$

$2.05407 E+C O$

$2.02268 E+C 0$
$1.492131+C 0$

I.o.8L IE+CO

- $5517 U E+C D$

$1.40111 t+C Q$

$1.77897 t+C 0$

$1.61023 t+C 0$

.1243UE+UU 2.1241AE+UD $2.13407 E+C O$

$.04100 E 400$ 2.04689E+UU

(2.05975E+00

2.02074t00

l. $469216+00$

$1.940671+00$

$2.00546 t+00$

$2.034 H 1 t+00$

2.00SOOE +00
$1.97601 \mathrm{t}+00$

$2.30218++C 0$

2.07rizeor

$2.01144 E+C O$

$1.48281 E+C$

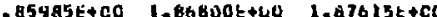

$1.83448 E+00$

1. $80987 t+00$

1. $842446 \times 00$

$1.81+646+40$

$1.07613 E+C 0$
$1.85140 E+00$

1. $78163 t+c 0$

$1.7405 B E+00$

1.75U20E*00

$1.67703 \mathrm{E}+00$

$1.65831 t+u 0$

$.7081 B E+40$

i.66AYSEtuU

1.65U35E*UD

$1.60362 \mathrm{E}+00 \quad 1.61313 E+00$

$1.586475+00$

. $37075 E+00$ i.54A247t+0

$1.55516 E+00$ 1.56707t+00

$\begin{array}{ll}1.54017 E+00 & 1.55227 E+00 \\ 1.52578 E+00 & 1.53306 t+00\end{array}$

$1.51351 E+C D$

$1.49954+C O$

$1.47332 \mathrm{E}+\mathrm{CD}$

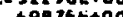

$1-53 B 06 t+00$
$1.52443 E+00$

$1.51136 t+00$

$1.49085 E+00$

$\begin{array}{lll}-46205 E+C U & 1.47396 E+00 & 1.48687 t+00 \\ (.44932 t+C O & 1.46237 E+00 & 1.47543 t+00\end{array}$

$1.43811 t+C O \quad 1.45131 E+00 \quad 1.43450 k+U 0$

$1.42742 E+C 0$

$1.44075 E+00$ 1.45407E+DO

1.417232400

$1.398315+c$

$\begin{array}{ll}1.43068 E+00 & 1.44413 E+00 \\ 1.42110 E+00 & 1.43467 E+40\end{array}$

1. $42110 E+00$ 1.43467E+00

$1.41149 E+00$
$1.40333 E+00$
$1.4171116+00$

$1.40333 E+00$

$1.417116+00$

1.75962E+00

L. $71833+\div 00$

1.6795460

$1.66122 E+00$

$1.64351 t+00$

$1+b r 644 t+C 0$

$1.59414 E+00$

1.57898E+CO

$1.55033 \mathrm{E}+00$

$1.33087 \mathrm{E}+00$

$1.51161 t+00$

$1.49979 E+00$

$1.47164 E+00$

$1.46739 E+00$

$3=45758 E+00$

$l=44423 \mathrm{E}+00$
$J .43434 E+00$

i. $43090 \mathrm{k}+00$

$1.43090 k+00$

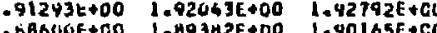

L. 42 RA LE+CO
$2.5824 C E+00 \quad 2.38160 E+00$

2.5COL $6 E+00$

2.45996E+00 2.46073E+00

2.42084F+00 2.6221 $3 E+00$

(2.3es43etod

$3.30315+00$ 2.34755E+00

$2.273165+000027633 E+00$

$2303 t E+0 U$ 2.24197E+0O

.20434t+00 2.2CE43E+40

$2.13093 E+00 \quad 2.17573 E+00$

$2.10747 E+00 \quad 2.11276 E+00$

$2.06248 k+00$

2.05300E+UO

Q40

1.9620

$93542 E+0 C$ 1.94241E +00

1.

$1.4443 C 1+40 \quad 1=05245 t+00$

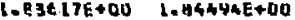
$1=81321 k+00$ L.8222AE+0U

- PICG7E+00 L-4GO32E+00

1. 7 7907e+00

1.

$1.709025+00$ 1.71g42E

$-49023 t+40 \quad 1.70007 t+00$

$1.67260 t \rightarrow 00 \quad 1.682926+00$

.654EIE+UU J.A65TOE+UO

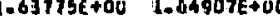

$.62 .153 E+00$ 1.633u4E+00

L.60571E+DO N.61763E+OD

- $44039+40$ 1-tc280E+vo

lasas6E+00

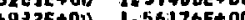

$536785+001.540186+00$

$52438 k+00$ i.53714E+00

$51276 E+00$ 1.525t2E+00

$1.51460 E+00$

$1.49089 E+00$ L.50408E+00

1.49404L+00

-4ricse+0O l-4EAHBE+0O

$45302 t+00$ l. ThS

$1.44469 E+00 \quad 2.45847 E+00$
$1.49678 E+00 \quad 1.45068 E+00$ 
GAMMA $=1+(1-h)(2 S R /(1-P H I)-1)(1-P H I)+45+W(2 S W-2)$

PH!

\#2.40000E-01 2.50000E-Cl $2.60000 E-C 2$

2.80000E-01 2.90000E-01

2.58060E+00

$1.00000 E-02 * 2.54019 E+00$

$2.00000 E=02 * 2.50042 E+00$

$4.00000 \mathrm{E}-02 \div 2.42343 \mathrm{E}+00$

$5.00000 \mathrm{E}-02: 2.38619 \mathrm{E}+00$

$6.00000 E-02 * 2.34979 k+00$

$7.00000 E-02: 2.31423 E+00$
$0.00000 E-02: 2.27949 E+00$

$9.00000 \mathrm{E}-02 * 2.24558 \mathrm{E}+00$

$1.00000 E=01 * 2.21248 E+00$

$1.20000 \mathrm{E}-01 * 2.14872 \mathrm{E}+00$

$1.30000 \mathrm{E}-01 * 2.11805 \mathrm{t}+00$

$1.400 N N E-01 * 2.08816 E+00$

I. $50000 \mathrm{E}-01 * 2.05907 \mathrm{Z}+00$

$1.60000 \mathrm{E}-01 * 2.03075 \mathrm{E}+00$

$1.70000 \mathrm{E}-01+2.00321 \mathrm{E}+00$

$1.80000 \mathrm{E}-01+1.97643 E+00$
$1.90000 \mathrm{E}-01 * 1.95041 \mathrm{E}+00$

$2.00000 E-01 * 1.925 \mathrm{LEE+00}$

$2.10000 E-01 * 1.90060 E+00$

$3.30000 \mathrm{E}-01.1 .05371 \mathrm{t}+00$

$2.400005-01 * 1.03134 E+00$

$2.50000 E-01 * 1.90967 E+00$

2.60000E-01 1.78869E+0O

$2.70000 t-01+1.76840 \mathrm{t}+0$

.80000E-01 *1.74877E+00

$2.90000 t-01 * 1.72981 t+00$
$3.00000 E-01 * 1.71150 E+00$

$3.000 D O E-01$
$3.10000 E-01$
$3.711150 E+00$

$3.20000 E-01 * 1.67680 E+0$

$3.30000 \mathrm{E}-0 \mathrm{OL}: 1.66038 \mathrm{t}+0$

$3.40000 \mathrm{E}-01$
$3.50000 \mathrm{E}-01$
$3.61 .64456 \mathrm{E}+1.62435 \mathrm{E}+00$

$3.60000 E-01+1.61471 E+00$

$3.70000 \mathrm{E}-\mathrm{OL}: 1.60065 \mathrm{E}+00$

3.60000E-01 1.54715E+00

$3.90000 \mathrm{E}-01$ 1.57420E+00

$4.00000 \mathrm{E}-01+1.56179 \mathrm{t}+00$

$4.10000 \mathrm{E}-01$
$4.20000 \mathrm{E}-0 \mathrm{1}$
$4.54990 \mathrm{1}+00$

4.20000 E-0L $1.53853 E+00$
4.30000 E-01
$4.52766 E+00$

$4.30000 E-01 * 1.52766 E+00$
$4.40000 E-01 * 1.51727 E+00$

$4.40000 \mathrm{E}-01 * 1.51727 \mathrm{E}+00$
$4.50000 \mathrm{E}-01 * 1.50737 \mathrm{E}+00$

$4.60000 E-01 * 1.49792 E+00$

$4.70000 E-01 \div 1.4 B 893 E+00$

$4.80000 \mathrm{E}-01 * 1.48038 \mathrm{E}+00$

$4.90000 \mathrm{E}-01: 1.47226 \mathrm{E}+00$

$5.00000 \mathrm{E}-01: 1.46455 \mathrm{E}+00$

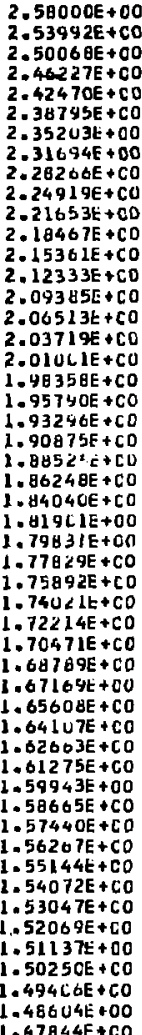

$2.53965 E+C O \quad 2.53939 E+0 D$

$2.57760 E+00$

$2.30093 E+C O$ 2.50119E+0O $2.50145 E+00$

$\begin{array}{lll}2.46304 E+C O & 2.46301 E+00 & 2.40458 E+00 \\ 2.42597 E+C O & 2.42724 E+00 & 2.42851 E+D O\end{array}$

$2.38971 E+C O \quad 2.39147 E+0$

$2.35427 E+C O \quad 2.35651 E+0$

(2.325DE+UO

$2.252400+C D$ 2.2564IE+CO $2.200 D 2 E+0 O$

$2.22057 E+C O \quad 2.22462 E+00$

2.18914t+CU $2.19361 t+00$

$2.15849 E+C U 2.16338 E+0 O$

2.16826E+00

$2.13420 t+0$

$2.099531+\mathrm{CO} 2.10521 k+00$

$2.0 n+33 t+0$

$2.04363 t+C U \quad 2.05007 E+00 \quad 2.05050 E+C O$

$2.01681 \mathrm{~L}+\mathrm{CU}$ 2.0236lt +00

$2.03441 \mathrm{E}+00$

2. OUbO4t +00

$.46540 t+C U$ 1. $47289 E+00$

$1.940796+C$

$1.96039 t+00$

$1.43120 \mathrm{E}+0 \mathrm{C}$

$1.90220 \mathrm{E}+00 \quad 1.91065 \mathrm{E}+0$

. $87124 k+C 0 \quad 1.88001 t+00$ l.

$1 . B 2836 \xi+C 0$

$1.007945+C 0$

$1.78817 t+C 0$

$1.76907 t+C 0$
$1.75 C 61 t+C 0$

$1.73278 E+C O$

$1.71558 E+C U$

$1.69899 E+C O$

$1.683 C U t+C 0$

$1.66760 E+C O$

$1.63854 E+C O$

$1.32485 E+C O$

$1.61170 t+00$

$1.59909 E+C O$

$1.58701 E+C O$

$1.57543 E+C 0$

$1.55377 t+C u$

$1.54366 \mathrm{t}+\mathrm{CO}$

1. $3401 \mathrm{C}+\mathrm{CO}$

$1.51607 \mathrm{E}+\mathrm{CO}$

$1.50774 t+c 0$

$1.49983 E+C 0$

$1.44232 E+C O$

\begin{tabular}{|c|c|c|}
\hline 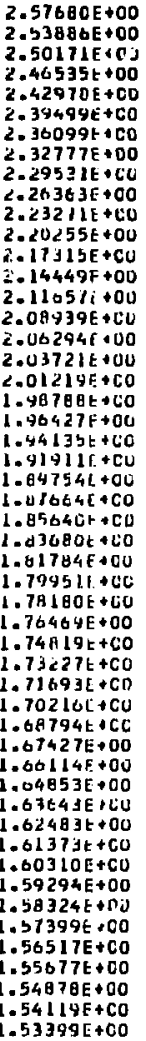 & 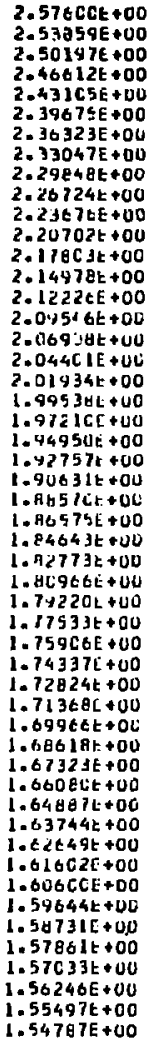 & 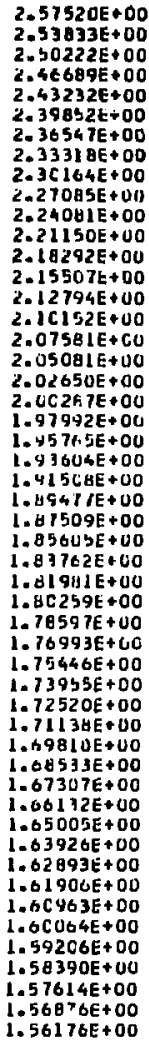 \\
\hline
\end{tabular}

1. $04705=+0$

l. $d<71 a t+0 C$

1. 798 UOE +00 1.80745t+60

$1.77922 E+00$ 1.78436E+CO

1.7SI UOE+OO $1.771401+00$

1.74342Etud $1.73400 \mathrm{E}+00$

1.7 $1.73322+00$

1. TOUE+ $1.72118 E+00$

1.60502t+uO

1.66450E+100 1.67622t+C

$1.63694 \mathrm{E}+00 \quad 1.64904 \mathrm{E}+00$

$1.62398 \mathrm{E}+00 \quad 1.63625 \mathrm{E}+0 \mathrm{O}$

$1.62398 E+00$

$1.59962 \mathrm{E}+00 \quad 1.61222 \mathrm{E}+00$

. 58620 t. $00096 \mathrm{E}+00$

1.57727E+UO $1.59019 k+0$

1. $55683 E+00$ 1.57989E+U

$.57005 t+00$

$1.52142 E+00$ 1.54320E+

$1.52142 E+00 \quad 1.53510 E+0$

$1.506211+00$ 1.52010E+00

1. $54787 E+00$ 
GAMHA $=1+(1-W)(2 S R)(1-P H I)-1)(1-P H I) * 5+W(25 w-2)$

$$
S R=1.30 \quad S W=1.76
$$

OHI

W.20000E-01 3.30000E-01 3.40000E-01 3.50000E-01

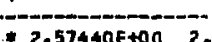

0. $2.57440 E+002$

$2.00000 \mathrm{E}-02 \div 2.50248 \mathrm{E}+00$

$3.00000 \mathrm{E}-\mathrm{d2}+2.46766 \mathrm{E}+00$

$.00000 E-02 \div 2.43359 E+00$
$3.00000 E-02$

$6.00000 E-02+2.34771 E+00$

7.00000 E-02 $* 2+335$ B9E+0O

$.00000 E-02+2.30481 E+00$

9.00000 E-02 $* 2.27446 E+00$

1.00000E-0I $* 2.24485 E+00$

$1-10000 E-01 * 2.21597 E+D 0$

$1.20000 \mathrm{E}-\mathrm{OI} * 2.18780 \mathrm{E}+00$

$1.40000 \mathrm{E}-01.2 .13362 \mathrm{E}+00$

1.50000 E-01 2,10759E+00

1.60000 E-01 $* 2.08225 E+00$

$1.70000 \mathrm{E}-01+2.05762 \mathrm{k}+0 \mathrm{D}$

1.60000E-01 2.03365E400

$1.90000 E-01$ 2.01036E+00

2.00000E-01 1.98775E+00

2.10000E-OL 1.96580E+0

$2.20000 E-01$ 1.94450E+00

2.30000E-01 1.92384E+O

2.40000E-01 1.90383E+0

2.50000E-DI 1.68444E+00

$2.60000 E-01 * 1.86567 E+00$

2.70000E-01 1.84751E+00

$2.80000 E-01+1.82996 E+00$
$2.90000 E-01$
3

2.90000E-01 $1.61299 E+00$

3.00000E-01 1.79661E+00

$3.10000 \mathrm{E}-\mathrm{Cl} 1.78080 \mathrm{E}+0$

3.20000E-01 1.76556E+00

$3.30000 E-01 * 1.75086 E+00$

$3.40000 E-O 1$ 1.73671E+00

$3.50000 E-01$ 1.723 IOE+OO

$3.60000 E-01+1.71001 E+00$

3.70000E-01 $1.60743 \mathrm{E}+00$

$\begin{aligned} & 3.80000 E-01 \\ & 3.90000 E-01\end{aligned} 1.68535 E+00$

3.90000 E-01 $+1.67376 t+00$
4.00000 E-01 $1.66266 E+00$

$4.00000 E-01$
$4.10000 E-01$
$4.620000202 E+00$

$4.20000 \mathrm{E}-01 * 1.64184 \mathrm{E}+00$

$4.30000 E-01 * 1.63212 E+00$
$4.40000 E-01 * 1.62262 E+00$

$4.40000 E-01$
$+.50000 \mathrm{E}-02+1.61396 \mathrm{E}+00$

$4.60000 \mathrm{E}-01$ 1.605S1E+00

4.70000E-01 1.59747E+00

$4.90000 \mathrm{E}-01.3 .58255 \mathrm{E}+00$

$4.90000 \mathrm{E}-01$
$.00000 \mathrm{E}-0 \mathrm{~L}$

$.57360 E+C O$

$.53779 E+00$

$2.50274 E+C O$

$2.434 U 6 E+C O$

$2=40204 E+C O$

$2.36995 E+C O$

$2.338608+00$

$2.30797 E+C$

$2.27807 E+C O$

$2.24890 E+C O$

$2.22044 E+C O$

$2.192696+00$

$2.16565 E+C O$

$2.13930 E+C$

1136SE+CO

$.08869 E+C O$

$.06441 E+C O$

$.04080 E+C D$

.01786E+ +

.97335E+0O

S6E $+C 0$

$.91289 \mathrm{E}+\mathrm{C}$

$.87529 E+C O$

$1.85740 E+C O$

$.82334 E+C O$

1. $-00725 E+C O$

$1.79167 \mathrm{E}+00$

$1.77665 E+00$

$.76217 E+C O$

$.734 \mathrm{B2E}+00$

. $72192 E+00$

$.70952 \mathrm{E}+\mathrm{CO}$

$1.686 \dot{1} \mathrm{iE}+C \mathrm{CO}$

$1.67526 E+C O$

$.66478 E+C O$

$1.65476 E+00$

$1.64517 E+C O$

(1)

$1.61896 E+00$

1.61203E+00

$\begin{array}{ll}1.60349 E+C 0 & 1.61717 E+C 0 \\ 1.59633 E+C 0 & 1.61012 E+C O\end{array}$

$1.60342 E+C O$

$50300 E+C O \quad 2.50325 E+00$

$2.46920 \mathrm{E}+\mathrm{CO} 2.46997 \mathrm{k}+00$

$2.43613 E+C O \quad 2.43740 E+00$

$40380 E+C O$ 2.40556E+DO

$37219 E+C O \quad 2.37443 E+C D$

(2.17622 +00

$2.15067 \mathrm{E}+00$

. $09513 E+C O$ 2.10156E+00

07121E+co 2.07801E+00

$479 b E+c 0$ 2.05blOE+00

2.03285E+00

2.01123E+00

1.49025E+00

$1.96989 k+00$

$1.95015 E+00$

$1.90313 E+C O \quad 1.93101 E+00$

1. $88492 E+C a \quad 1.89474 E+d O$

- $86729 E+00$ 1. $67716 E+00$

$1.85025 E+00$ l. $86040 E+00$

1. $3379 E+C U$ 1.844118E+0D

- $1789 E+C O$ 1.82852E+00

$1.80254 E+C O$ I. $81341 E+00$

$1.77348 E+C U$ I. $784 B O E+O D$

. $75975 E+C O$ I.77127E+0O

$1.74654 E+C O \quad 1.75826 E+00$

1.73383E+CO $1.74574 k+00$

1.72162E+CO l.733 T2E+0O

1.722 L7E+00

1.6711

1.68787E+CO l.7004Bk+00

$1.69031 E+00$

1.6005E+00

1.67129t+00

1.66241E+00

1.6595E+00

$1.645862+00$

$1.63817 k+00$

$1.63085 \mathrm{E}+00$

$1.62391 E+00$ 
Table B5. Values of the BOTE model parameters $R_{B 0}, v_{g 1}(=\psi), V_{g 2}, P_{g 1}$, and $P_{g 2}$ for various values of $\gamma$.

\begin{tabular}{|c|c|c|c|c|c|c|c|c|}
\hline GAMMA & 4 & $1.10000 E+00$ & $1.150000+60$ & 1. $2000 C E+100$ & $1.25000 E+00$ & 1 - $10000 t+00$ & $1.350 C O E+00$ & $1.40000 E+00$ \\
\hline RSO & + & $7.78575 E-02$ & $0.41625 \bar{E}-01$ & $0.89670 E-01$ & $5.21383[-61$ & $4.60272[-01$ & $9.8 B A 7(\mathrm{E}-\mathrm{Cl}$ & 1. $01444 E+C C$ \\
\hline VS I & 4 & $2.13949 E-01$ & $2.3943 \mathrm{CE}-\mathrm{OI}$ & $2.421 s t t-L 1$ & $3.31468 \bar{c}-\mathrm{Cl}$ & $3.614481-01$ & $3.88963 \mathrm{E}-\mathrm{Cl}$ & $4.14593 \mathrm{E}-\mathrm{Cl}$ \\
\hline vS 2 & $*$ & $3.11430 E-01$ & $\therefore 3 t \in 5 C E-01$ & $3.35630 \mathrm{E}-\mathrm{Cl}$ & $3.71033 c-u 1$ & $3.841045-01$ & $3.45548 t-01$ & $4 . c 3>75 t-[1$ \\
\hline PS 1 & * & $4.35947 t-02$ & $t .2 \mathrm{E} 50 \mathrm{Ct}-\mathrm{U}_{\mathrm{c}}$ & A. UBCIRE-LZ & $5.10633 \hat{c}-v 2$ & $1.13004 t-01$ & $1.247>9 t-1: 1$ & $1.43240 \mathrm{E}-\mathrm{Cl}$ \\
\hline PS2 & 4 & $9.23702 \mathrm{t}-\mathrm{C2}$ & 1. $\cos 2 \theta t-U 1$ & L. $14 \times 751:-01$ & $1.2236+6-61$ & $1.2424>5-02$ & $1.2] 25 \in[-61$ & $1.37211 E-C 1$ \\
\hline
\end{tabular}

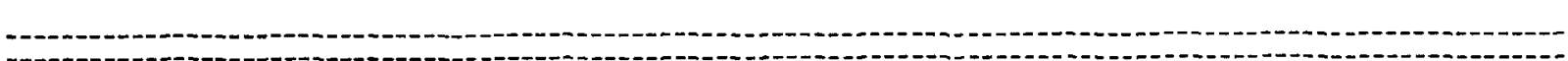

\begin{tabular}{|c|c|c|c|c|c|c|c|c|c|}
\hline & GANNA & $\downarrow$ & $1.450005+00$ & $1.5 U 000 E+U C$ & 1.55COUE : CU & $1.6000 C t+C C$ & $1.1,000 i+00$ & $1.700 C C E+C O$ & 1.15UULE +CO \\
\hline & RSO & 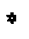 & $1.03767[+00$ & 1.C5905t+UL & I. C7842t+1.C & $1.09754 t+60$ & $1,11512 t+00$ & $1.13179 E+C O$ & $1.141 E S E+C E$ \\
\hline & VS 1 & 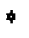 & $4.38739 E-C L$ & $4.0 \mid C \forall E E-U 1$ & $4.636 ., U E-L 1$ & $5.147435-61$ & $3 .<22431:-01$ & $5.451 U \perp E-C 1$ & $5.44444 E-C l$ \\
\hline & VS ̈̈ & $*$ & $4.15067 E-C 1$ & $4.23 t \cdot 1 \mathrm{eE}-\iota 1$ & $4.31 b e 7[-C 1$ & $4.39 \mathrm{Cl} / \mathrm{t}-\mathrm{Cl}$ & $4.40046 t-01$ & $4.52711 t-01$ & $4.54 W / 7 E-C 1$ \\
\hline & PS I & $\downarrow$ & $1.57136 \mathrm{E}-01$ & $1.7 \operatorname{cj} 23 E-C 1$ & $1.8 .14134[-61$ & 1. $\not<C 1<t-u l$ & $2.11+2121-01$ & $2.2 u \mid c L t-l: 1$ & $2.31711 \mathrm{E}-\mathrm{Cl}$ \\
\hline & PS? & 6 & $1.40637 E-01$ & $1.43562 \mathrm{E}-\mathrm{Cl}$ & $1+0,(6)-C) E-C 1$ & $1.4+1250 k-61$ & $1.301561-01$ & $1.51617 t-11$ & $1.93274 E-61$ \\
\hline
\end{tabular}




\begin{tabular}{|c|c|c|c|c|c|c|c|}
\hline GAMra & - $1.80000 E+00$ & $1.85 \angle O O E+C L$ & 1. 9CCUUE +CO & $1.95 C O U E+L C$ & $2.00000 E+C 0$ & $2 . C 5 O U C E+C C$ & $2.10000 E+C O$ \\
\hline RSO & - $\quad 1.16292 \mathrm{E}+00$ & $1.17755 k+0 C$ & $1.191+4 E+20$ & $1.20527 E+00$ & $1.21846 E+00$ & $1.23127 t+c 0$ & $1.24373 E+C O$ \\
\hline VSI & $* \quad 5.83355 E-01$ & t.. $61873 \mathrm{E}-01$ & $6.2 C C 49 E-C l$ & $t .37924 E-01$ & $0.55529[-01$ & $6.72694 E-61$ & $6.90043 E-C 1$ \\
\hline vs? & - $4.6 b 167 E-C L$ & $4.71 C 18 E-C 1$ & $4.7605 \mathrm{HE}-\mathrm{Cl}$ & 4. $82107 E-C 1$ & $4.87396[-01$ & $4.425101-01$ & $4.37492 t-C 1$ \\
\hline PS 1 & $6 \quad 2.43073 \mathrm{~F}-01$ & $2.54211 E-L 1$ & $2 . t 5140 E=02$ & $2.758962-01$ & $2.86479[-01$ & $2.96909 E-C 1$ & $3.07199 E-C l$ \\
\hline PS2. & - $1.54557 t-01$ & $1.5569 C E-U 1$ & 1. $5 t e 01 t-01$ & $1.57574 t-c 1$ & $1.58363[-01$ & $1.5 \sqcup C b 9 E-[1$ & $1.54077 E-C 1$ \\
\hline
\end{tabular}
WHERE

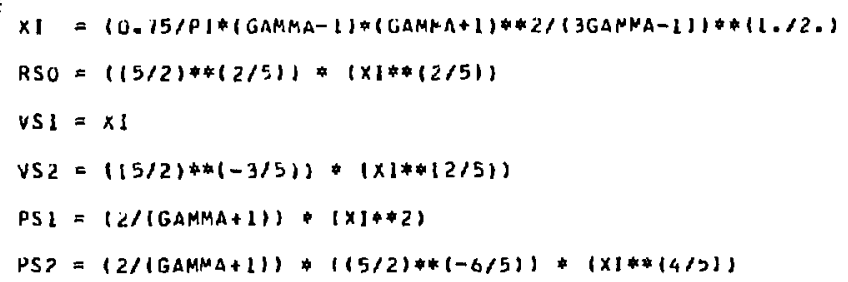

\begin{tabular}{|c|c|c|c|c|c|c|c|}
\hline GAMPA & $1 * 2.15$ & $2.20000 E+00$ & $2.2500 C E+O C$ & 2. $100 \mathrm{COE}+00$ & 2. $15000 E+00$ & $2.40000 t+00$ & $2.45000 E+0 C$ \\
\hline HSO & $4 \quad 1.25586 E+00$ & $1.2677 \mathrm{CE}+0 \mathrm{~L}$ & $1.27926 E+00$ & $1.2 \triangle C D 7 E+C O$ & $1.30164[+00$ & $1.31249 E+O C$ & $1.32314 E+C O$ \\
\hline vS1 & 7.064 Y6E-01 & $7.23773 \mathrm{E}-01$ & $1.4 C 340 E-6)$ & $7.56860 \hat{\mathrm{t}}-0 \mathrm{I}$ & $7.731975-01$ & $1.89411 E-C 1$ & $\theta . C 5 b 12 E-C 1$ \\
\hline vṣ 2 & $\$ 5.02346 \mathrm{E}-0 \mathrm{l}$ & $5.07081 E-01$ & $5.11706 \mathrm{E}-01$ & $5.16229 t-01$ & $5.20657 \mathrm{E}-01$ & $5.24 .997 E-02$ & $5.29255 E-C 1$ \\
\hline PS1 & - $\quad 3.17361 \mathrm{E}-01$ & $3.27405 \mathrm{E}-\mathrm{U} 1$ & $3.37340 E-01$ & $3.47174 E-01$ & $3.5691 \supset E-01$ & $3.66570 E-C 1$ & $3.7 E 145 \mathrm{E}-\mathrm{CI}$ \\
\hline PS 2 & $* 1.60223 E-01$ & $1.60707 E-01$ & $1.61134 E-U 1$ & $1.6151 O E-C 1$ & $1.61841 E-01$ & $1.62131 t-01$ & $1.62383 t-01$ \\
\hline
\end{tabular}




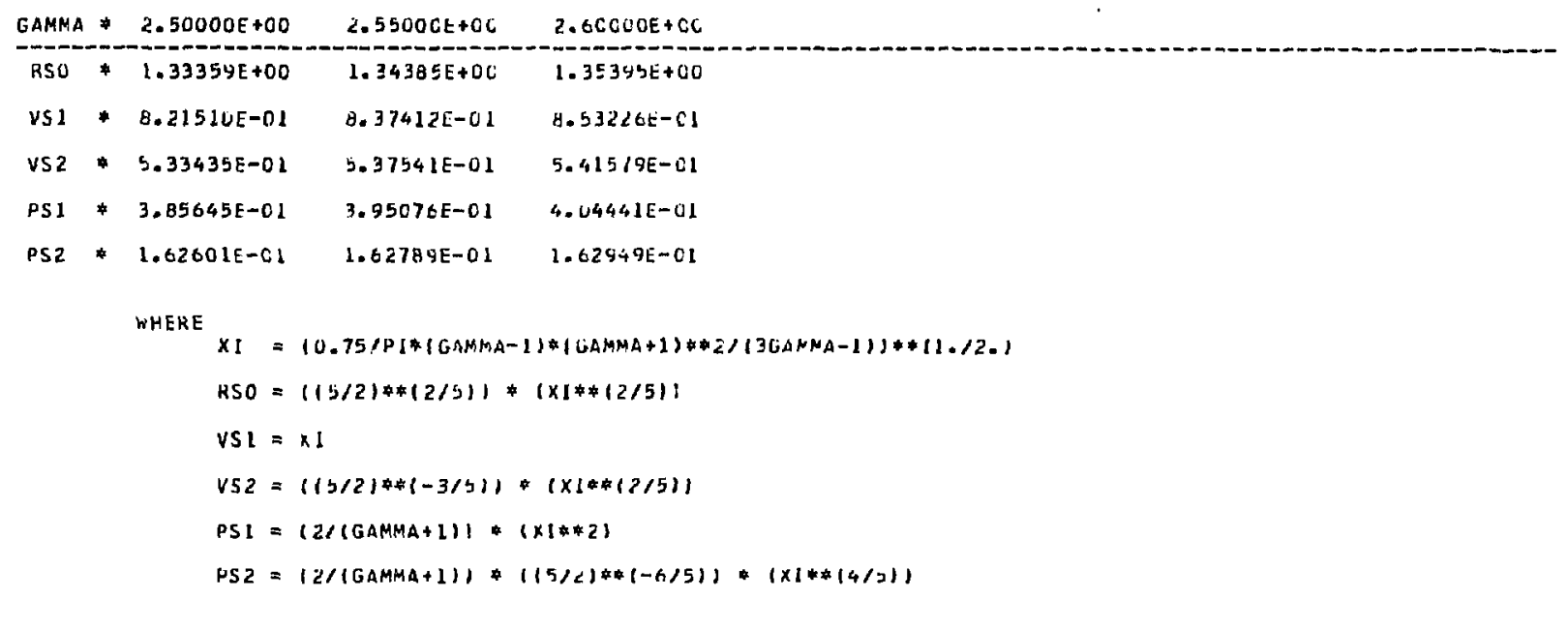

